\title{
Attosecond electron dynamics in molecules
}

\author{
Mauro Nisoli, ${ }^{*},+, \neq$ Piero Decleva, " Francesca Calegari,,,$\|, \neq$ Alicia Palacios, ${ }^{\perp}$ and \\ Fernando Martín $*, \perp, \#, @$ \\ †Department of Physics, Politecnico di Milano, Milano (Italy) \\ $\ddagger$ Institute for Photonics and Nanotechnologies, IFN-CNR, Milano (Italy) \\ IDipartimento di Scienze Chimiche e Farmaceutiche, Universitá di Trieste and IOM- \\ CNR, Trieste (Italy) \\ $\S$ Center for Free-Electron Laser Science, DESY, Hamburg, Germany \\ \|Department of Physics, University of Hamburg, Hamburg, Germany \\ $\perp$ Departamento de Química, Módulo 13, Universidad Autónoma de Madrid, Madrid (Spain) \\ \#Instituto Madrileño de Estudios Avanzados en Nanociencia, Madrid (Spain) \\ @ Condensed Matter Physics Center (IFIMAC), Universidad Autónoma de Madrid (Spain)
}

E-mail: mauro.nisoli@polimi.it; fernando.martin@uam.es 


\section{Contents}

1 Introduction $\quad 5$

2 Generation of attosecond pulses $\quad 9$

2.1 High-order harmonic generation . . . . . . . . . . . . . . . 9

2.2 The semiclassical three-step model $\ldots \ldots \ldots \ldots \ldots$

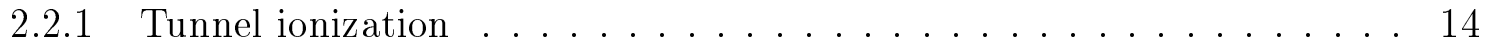

2.2.2 Motion of the electron in the driving field and recombination . . . . . 15

2.3 Semiclassical formulation of the three-step model based on strong-field ap-

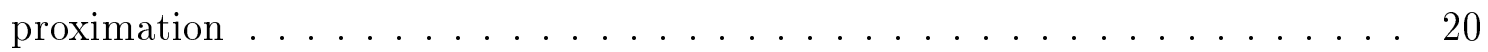

2.4 Generation of attosecond pulses . . . . . . . . . . . . . . . 24

2.4.1 Attosecond pulses in the water-window region . . . . . . . . . 27

2.4.2 EUV pulses with circular polarization . . . . . . . . . . . 28

2.5 Isolated attosecond pulses $\ldots \ldots \ldots \ldots \ldots \ldots$

2.5.1 Brief overview of femtosecond laser technology . . . . . . . . . . . . 29

2.5.2 Generation of isolated attosecond pulses . . . . . . . . . . . 32

3 Attosecond experimental techniques 35

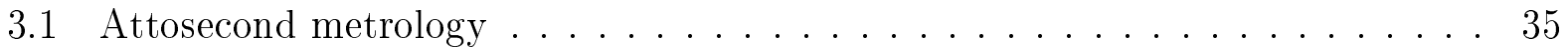

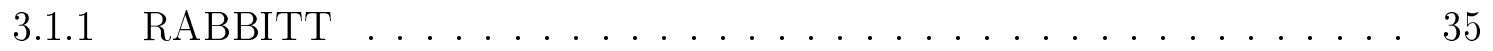

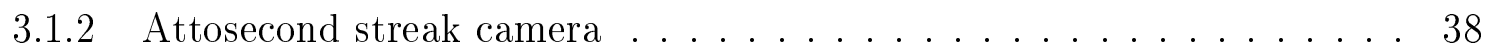

3.1 .3 Optical methods . . . . . . . . . . . . . . . . 43

3.2 Attosecond spectroscopy techniques . . . . . . . . . . . . . . . 45

3.2.1 Attosecond electron/ion spectroscopy . . . . . . . . . . . . . 47

3.2 .2 High-order harmonic spectroscopy . . . . . . . . . . . . . . . . 49

3.2.3 Attosecond non-linear spectroscopy . . . . . . . . . . . . 52

3.3 Attosecond experimental techniques without attosecond pulses . . . . . . . 55

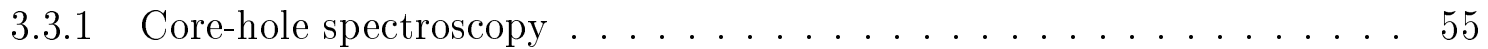


3.3 .2 Attoclock . . . . . . . . . . . . . . . . . . . 59

3.4 X-ray Free Electron Lasers . . . . . . . . . . . . . . . . . 62

4 Theoretical methods for attosecond science $\quad 65$

4.1 Molecular ionization . . . . . . . . . . . . . . 65

4.1.1 Time-dependent molecular wave packet $\ldots \ldots \ldots \ldots$. . . . 67

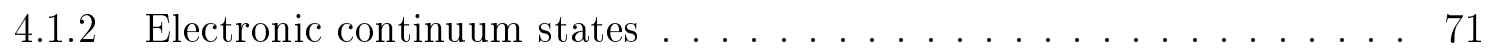

$4.1 .3 \quad$ Single Channel Approximation . . . . . . . . . . . . . . . . . 72

4.1 .4 Continuum Orbitals . . . . . . . . . . . . . . . . . . . 74

4.1.5 Computational Approaches to Continuum Orbitals . . . . . . . . . 76

4.1 .6 Coupled Channel approaches . . . . . . . . . . . . . 78

4.1.7 The B-spline static exchange DFT method . . . . . . . . . . . . . 79

4.2 Charge Migration . . . . . . . . . . . . . . . . 81

4.2.1 Methods based on the sudden approximation . . . . . . . . . 82

4.2.2 Methods explicitly including the ionization step . . . . . . . . 86

4.3 Nuclear degrees of freedom . . . . . . . . . . . . . . . 88

4.4 HHG methods . . . . . . . . . . . . . . . . . . . 92

5 From diatomic to polyatomic molecules $\quad 96$

5.1 Attosecond pump-probe techniques applied to hydrogen molecules . . . . . . 97

$5.1 .1 \quad$ EUV-pump/IR-probe $\ldots \ldots \ldots \ldots \ldots \ldots \ldots$

$5.1 .2 \quad$ EUV-pump $/$ EUV-probe . . . . . . . . . . . . . . . . . . 102

5.2 Beyond $\mathrm{H}_{2}$ : electron dynamics in more complex diatomics and small polyatomics 108

6 Towards sub-femtosecond electron dynamics in bio-relevant molecules 116

6.1 Experimental findings . . . . . . . . . . . . . . . . 117

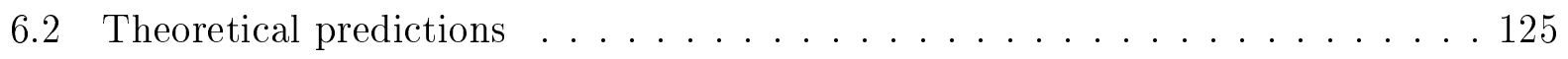

$\begin{array}{lll}7 & \text { Towards attosecond molecular imaging } & 141\end{array}$ 
7.1 Tomographic imaging of molecular orbitals . . . . . . . . . . . . . . 142

7.2 Laser-induced electron diffraction . . . . . . . . . . . . . . . 145

7.3 Time-Resolved Holography with Photoelectrons . . . . . . . . . . . . 148

7.4 Multichannel photoemission in strong field ionization . . . . . . . . . . . 149

8 Conclusion and outlook $\quad 152$

$\begin{array}{ll}\text { Acknowledgement } & 159\end{array}$

9 Abbreviations $\quad 160$

$\begin{array}{ll}\text { References } & 163\end{array}$ 


\begin{abstract}
Advances in attosecond science have led to a wealth of important discoveries in atomic, molecular and solid-state physics, and are progressively directing their footsteps towards problems of chemical interest. Relevant technical achievements in the generation and application of extreme-ultraviolet sub-femtosecond pulses, the introduction of experimental techniques able to follow in time the electron dynamics in quantum systems, and the development of sophisticated theoretical methods for the interpretation of the outcomes of such experiments have raised a continuous growing interest in attosecond phenomena, as demonstrated by the vast literature on the subject. In this review, after introducing the physical mechanisms at the basis of attosecond pulse generation and attosecond technology, and describing the theoretical tools that complement experimental research in this field, we will concentrate on the application of attosecond methods to the investigation of ultrafast processes in molecules, with emphasis in molecules of chemical and biological interest. The measurement and control of electronic motion in complex molecular structures is a formidable challenge, for both theory and experiment, but will indubitably have a tremendous impact in chemistry in the years to come.
\end{abstract}

\title{
1 Introduction
}

"Chemical reactions occur by the rearrangement of atoms, breaking and remaking bonds to form the final product species from the initial reactant molecules. ... this chemical transformation is a dynamical process involving the mechanical motion of electrons and atomic nuclei. These underlying elementary dynamical steps occur on the ultrafast time scales of molecular translations, vibrations, rotations, and electronic transitions, most conveniently measured in femtoseconds $\left(1 \mathrm{fs}=10^{-15} \mathrm{~s}\right)$. In this sense, all chemistry is femtochemistry. ${ }^{1 "}$ Born in the second half of the past century, femtochemistry is now a well-established scientific discipline. ${ }^{2}$ One of its goals is to control a chemical reaction by steering the motion of 
the nuclei of the involved molecules by using femtosecond light pulses. As reactivity is at the heart of chemistry, nowadays femtosecond lasers are widely used in most areas of chemical sciences.

With the advent of the twenty-first century, developments of coherent light sources permitted to create even shorter light pulses, down to a few tens of attoseconds $\left(1\right.$ as $=10^{-18}$ $\mathrm{s})$, in the extreme ultraviolet (EUV) spectral region. This impressive progress in laser technology, which will be discussed in the first two sections of this review, has opened the way to directly control the rapid motion of electrons inside a molecule and, as a consequence, the subsequent nuclear dynamics occurring on a longer temporal scale. Since the time-dependent electronic density is ultimately responsible for bond formation and bond breaking in molecules, controlling this motion has thus opened the door to a new way of doing chemistry, usually termed attosecond chemistry ${ }^{3-5}$ or attochemistry for short.

Attosecond pulses in the EUV spectral region were first employed for the investigation of ultrafast electron dynamics in atomic systems. Immediately after their experimental realization, ${ }^{6,7}$ they were used to measure Auger relaxation in krypton atoms. ${ }^{8}$ Shortly afterwards, other important applications were reported also in atoms, as the real-time observation of valence electron motion in krypton, ${ }^{9}$ the characterization of electron wave packets (WPs) in helium, ${ }^{10}$ the measurement of the delay in photoemission using isolated attosecond ${ }^{11}$ or trains of attosecond pulses, ${ }^{12}$ the reconstruction of a correlated two-electron wave packet ${ }^{13}$ and the build-up of Fano interferences ${ }^{14,15}$ in helium, or the analysis of the tunneling process of an electron ionized from noble gas atoms, ${ }^{16-18}$ to name but a few examples. In the last few years, attosecond technology has also been used to study ultrafast processes in condensed matter ${ }^{19-23}$ in order to understand physical phenomena that may enable the scaling of electronic and photonic circuits to atomic dimensions. ${ }^{24}$

Although there are fewer applications of attosecond technology to investigate ultrafast electron dynamics in molecules, their huge potential to understand a large variety of chemical processes has been recently realized. Indeed, ultrafast electron dynamics induced by light 
plays a crucial role in the early stages of photosynthesis, in radiation damage of biologically relevant molecules, and in general in any chemical or biological process in which electron transfer occurs. As recently shown, ${ }^{25}$ electron transfer along a molecular chain can be much faster than molecular vibrations and, therefore, requires attosecond time resolution for its understanding.

In addition to high time resolution, another very challenging aspect of attosecond studies in molecules is the involvement of many degrees of freedom, with complex and generally unknown interconnections. For example, electronic and nuclear degrees of freedom are usually coupled due to non adiabatic effects, and electron correlation plays a significant role. ${ }^{26}$ On top of that, EUV excitation in large molecules involves a large number of ionization channels due to the energy proximity of molecular orbitals in systems containing such a large number of electrons. Therefore, with attosecond sources, chemistry enters into new, never-considered, domains of light-matter interaction, which require the support from theoretical modeling right from the start. For this reason, the role of theory as a prerequisite to outline new experiments, and to disclose their feasibility and informational content, will be stronger than ever before. Until very recently, complete theoretical methods able to take into account the correlated motion of electrons and nuclei after interaction with attosecond pulses were practically limited to diatomic molecules, in particular $\mathrm{H}_{2} \cdot{ }^{27-32}$ Consequently, since the accomplishment of the first pump-probe measurement with attosecond temporal resolution in $\mathrm{H}_{2}$ and $\mathrm{D}_{2}$ molecules, ${ }^{33}$ only a few experimental applications of attosecond pulses to polyatomic molecules have been reported. These measurements, which constitute the central part of this review, have motivated extensive theoretical developments, which will also be described here.

Among all possible applications of attosecond science to molecular systems, a very exciting one is the investigation of charge motion along the molecular chain of biologically relevant molecules. In this review, particular attention will be devoted to this specific process, which, according to early theoretical predictions, can be driven by purely electronic effects ${ }^{34}$ and 
can precede any rearrangement of the nuclear skeleton because it takes place on a temporal scale ranging from few femtoseconds down to tens of attoseconds. ${ }^{25,34-37}$

The review paper is organized as follows. Section 2 introduces the basis of attosecond technology, starting from the description of the high-order harmonic generation (HHG) process in atomic gases, which leads to the production of attosecond pulses in the form of isolated pulses or trains of pulses. Attosecond photon pulses are always associated to attosecond electron pulses through the so-called re-colliding electron wave packets, which provide spatial resolutions down to the angström range, due to the short de Broglie wavelength of these electron wave packets. This is another key point in attosecond technology that will also be examined in section 2. Section 3 reviews the main experimental methodologies that have been developed to achieve attosecond temporal resolution. Section 4 is devoted to the discussion of existing theoretical methods for the investigation of electron dynamics in molecules after excitation by attosecond pulses. Section 5 reports on experimental and theoretical results in molecular science starting from the simplest molecule, $\mathrm{H}_{2}$, and going up to small polyatomic molecules: we will show why attosecond pulses are such powerful tools for the investigation and control of the ultrafast electronic processes in these systems after sudden removal of an electron by the absorption of high-energy photons. Section 6 describes how the experimental and theoretical tools described in the first part of the review have been applied to investigate electronic dynamics in biologically relevant molecules: aminoacids, polypeptides, DNA, etc. In section 7, attosecond molecular imaging techniques will be briefly introduced to illustrate how one can take advantage of the sub-angström spatial resolution attainable from attosecond pulses. In the last section of the review, the current status of attochemistry is summarized and an outlook of future investigations in this field is given. 


\section{Generation of attosecond pulses}

\subsection{High-order harmonic generation}

When an intense and short laser pulse is focused into a gas medium, the electronic response becomes highly nonlinear and high-order harmonics of the driving laser frequency can be generated. High-order harmonic generation (HHG) is now a widely used technique for the production of coherent extreme ultraviolet (EUV) radiation. The typical spectrum of highorder harmonics is characterized by a fall-off, of two or three orders of magnitude, from the 3rd to typically the 5th harmonic, followed by a plateau, where the harmonic intensities remain approximately constant. This region, which can extend up to several hundreds of harmonic orders, is followed by a sharp cutoff, as schematically shown in Fig. 1. Only odd harmonics of the fundamental frequency are generated for reasons of symmetry. Indeed, in a centrosymmetric medium (gas target) a reversal of the driving field must cause a reversal of the nonlinear polarization, which is the source of the harmonic emission. Therefore, considering an expansion of the nonlinear polarization in terms of the driving field, only the odd terms are present. The first experimental observation of the high-order harmonic plateau in HHG was reported by the end of the 1980s by McPherson and coworkers ${ }^{38}$ and by Ferray and coworkers. ${ }^{39}$ In the first case 1-ps pulses at $248 \mathrm{~nm}$, generated by a $\mathrm{KrF}^{*}$ laser system, were focused into a noble gas target produced by a pulsed gas jet, at an intensity of the order of $10^{15}-10^{16} \mathrm{~W} / \mathrm{cm}^{2}$. The highest harmonic observed was the seventeenth $(14.6 \mathrm{~nm})$ in neon. ${ }^{38}$ In the second case, harmonic generation was obtained in xenon, krypton and argon by a Nd:YAG laser at $1064 \mathrm{~nm}$, with a pulse duration of $30 \mathrm{ps}$. Harmonics as high as the $33 \mathrm{rd}$ harmonic order $(32.2 \mathrm{~nm})$ were produced in argon. ${ }^{39}$ The most important experimental result was the observation that the harmonic conversion efficiency falls slowly beyond the fifth harmonic as the order increases: this was the first clear experimental evidence of the generation of the harmonic plateau. By using shorter driving pulses $(<1 \mathrm{ps})$ considerable extension of the maximum photon energy was independently demonstrated, in 1993, by two 


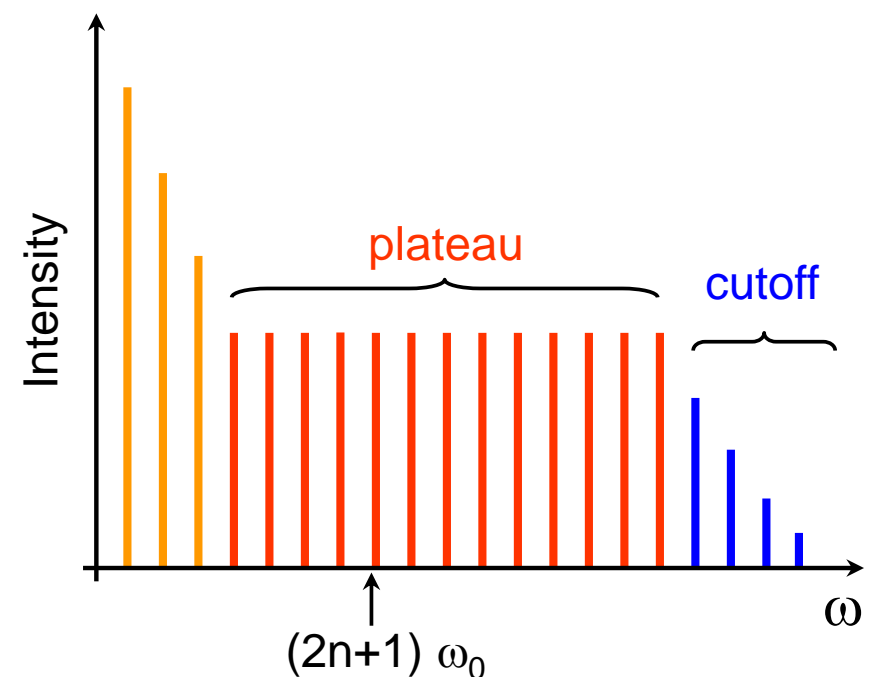

Figure 1: Schematic illustration of the spectrum produced by HHG in gases, showing the plateau region, where harmonic intensity remains roughly constant, and the cutoff region, where generation efficiency drops. In general, only odd harmonics of the fundamental frequency, $\omega_{0}$, are generated.

groups. ${ }^{40,41}$ By focusing on noble gas targets a 1-ps Nd:glass laser (1053 nm) at intensities between $10^{14}$ and $10^{15} \mathrm{~W} / \mathrm{cm}^{2}$, harmonics up to the 29 th in xenon, 57th in argon and at least up to the 135 th in neon were measured. ${ }^{41} 125$-fs laser pulses at 800 -nm were used by Macklin et al., with the observation of harmonics up to the 109 th order in neon gas. ${ }^{40}$

\subsection{The semiclassical three-step model}

In this section we detail a widely-employed semiclassical approach to explain in a simple way the physical process at the heart of HHG. A breakthrough in the theoretical interpretation of the physical mechanisms at the basis of HHG process was reported in 1992 by Krause et al., ${ }^{42}$ who first showed that the maximum photon energy achievable by HHG in gases follows the famous cutoff law: $\hbar \omega_{\max } \approx I_{p}+3 U_{p}$, where $I_{p}$ is the ionization potential of the gas and $U_{p}$ is the ponderomotive energy, i.e. the mean kinetic energy of an electron oscillating in the laser field. A simple semiclassical interpretation of the process was reported shortly afterwards in the framework of the so-called three-step or simple man's model, introduced in 1993 by Kulander et al., ${ }^{43}$ Schafer et al. ${ }^{44}$ and Corkum. ${ }^{45}$ It should be noted that a 
not well known precursor of this successful model is the so-called atomic antenna model proposed by Kuchiev in $1987,{ }^{46}$ which already contained the essential ingredients of the physical interpretation of the process. A relevant early work was also reported by Brunel, who investigated the interaction of intense electromagnetic waves with overdense plasmas ${ }^{47}$ and described the HHG process in terms of the fast varying plasma current generated by ionization, which takes place in a temporal interval localized around the maximum of the driving electric field, ${ }^{48}$ and by Corkum et al., who interpreted above-threshold ionization (ATI) occurring in parallel to HHG in terms of a simple semiclassical model. ${ }^{49}$

The semiclassical interpretation of the harmonic generation process is simple and can be easily understood with the help of Fig. 2. When a femtosecond laser pulse (also called driving pulse) is focused on a gas target, with peak intensity in the range between $10^{13}$ and $10^{15} \mathrm{~W} / \mathrm{cm}^{2}$, the Coulomb potential experienced by the outer-shell electrons is strongly modified by the laser electric field (indicated in Fig. 2 by a red line). A potential barrier is generated, through which an electron can tunnel, as shown in the first panel of Fig. 2 (blue dashed box). The probability of electron tunneling is high only in a narrow temporal region around each crest of the electric field. Indeed, as it will be discussed in Sect. 2.2.1, the tunnel ionization rate depends exponentially on the field amplitude. Therefore, the first step in the process of HHG has a pure quantum mechanical origin: tunnel ionization. After tunnel ionization, the electron is free to move in the continuum under the effect of the electric field of the driving pulse and its motion can be well described by using classical arguments (Newton's second law), at least as a first order approximation. The freed electron is first accelerated by the linearly polarized driving field away from the parent ion, until the electric field changes sign and thus the electron is decelerated, then reverses its direction of motion and is finally driven back towards its original position, as shown by the second panel in Fig. 2 (green dashed box). This electron is usually called recolliding electron. The time spent by this electron in the continuum, between the ionization and the recollision instants, depends on the particular trajectory it follows. If the recolliding electron recombines with the parent 


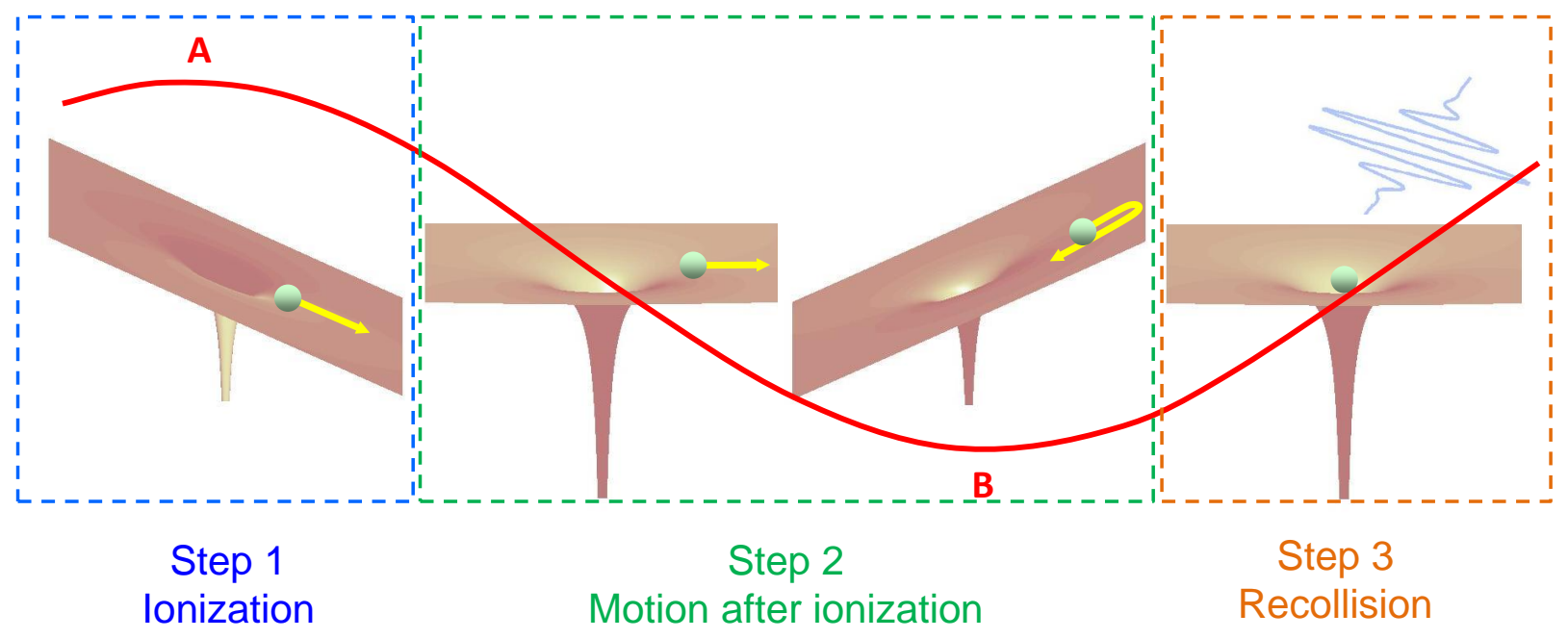

Figure 2: Three-step model. The electron is set free near a field crest by tunnel ionization (step 1, blue dashed box), then it is accelerated by the driving electric field and returns to its initial position (step 2, green dashed box), where it can recombine with the parent ion leading to the emission of an attosecond EUV pulse (step 3, orange dashed box).

ion, the kinetic energy acquired during its motion in the continuum can be released in the form of a burst of high-energy photons with sub-cycle duration, as shown in the third panel of Fig. 2 (orange dashed box). Depending on the intensity and wavelength of the driving field, the spectrum of this burst of photons may extend in the EUV from a few $\mathrm{eV}$ to a few hundreds of eV. As shown in Fig. 2(c), the electron set free around the peak crest indicated by letter A recollides with the parent ion near the first zero of the driving field following the peak crest designed by letter B. The entire process (ionization, motion in the continuum and recombination) is repeated every half optical cycle, since an electron can be freed around any of the field crests, thus leading to the generation of a train of attosecond pulses separated by half optical cycle of the driving radiation.

The EUV emission occurring during the third step of this model can be easily described in terms of the radiation emitted by an oscillating dipole, as illustrated in Fig. 3. During the first step of the HHG process a portion of the electron wave function, $\psi_{c}$, tunnels and propagates in the continuum under the action of the driving electric field. This continuum 

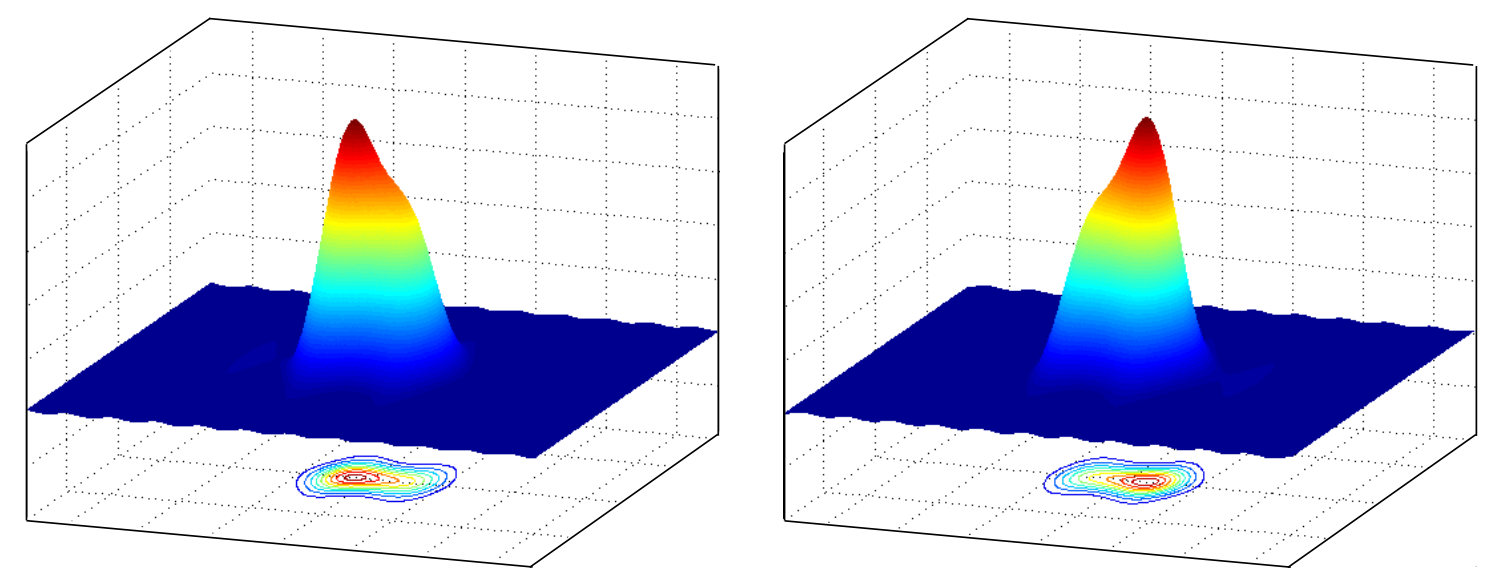

Figure 3: Total electron density distribution $\left|\psi_{b}+\psi_{c}\right|^{2}$ in two different instants. The main peak is related to the electron density associated to the bound component of the wave packet $\left(\psi_{b}\right)$, while the propagating plane wave refers to the continuum wave packet component $\left(\psi_{c}\right)$. The contour plots show the center of mass of the electron density distribution.

component of the electron wave packet, shown as a plane wave in Fig. 3, interferes with the component that remains bound to parent ion, $\psi_{b}$ (the pronounced peak in Fig. 3). The coherent overlap of these two components generates a dipole. Figure 3 shows the total electron density distribution $\left|\psi_{b}+\psi_{c}\right|^{2}$ in two different instants. As shown by the contour plots lying below the three-dimensional plot of the electron density, the center of mass of $\left|\psi_{b}+\psi_{c}\right|^{2}$ oscillates back and forth, thus giving rise to an electric dipole, which oscillates as the continuum wave function propagates. This electric dipole acts as an atomic antenna ${ }^{46}$ lasting just a small fraction of an optical cycle of the driving radiation, thus leading to high harmonic radiation.

In the following section we will briefly discuss the tunnel ionization process, then we will study the motion of the freed electron in the continuum by using classical Newton's equations and finally we will calculate the kinetic energy of the recolliding electron and therefore the energy of the generated EUV photons. We will show that, in spite of its simplicity, the model allows one to derive the cutoff law and the main characteristics of the quantum trajectories followed by the electrons in the temporal interval between the ionization and the recollision events. 


\subsubsection{Tunnel ionization}

In 1965 Keldysh formulated a theory to describe ionization of a one-electron atom exposed to strong laser radiation for the case of photon energies much smaller than the ionization potential of the atom. ${ }^{50} \mathrm{~A}$ dimensionless parameter, known as Keldysh parameter, was introduced to obtain a classification of different ionization mechanisms. The Keldysh parameter is expressed as

$$
\gamma=\omega_{0} \tau_{T}=2 \pi \frac{\tau_{T}}{T_{0}}=\sqrt{\frac{I_{p}}{2 U_{p}}}
$$

where $\omega_{0}$ is the angular frequency of the driving radiation, $T_{0}$ the corresponding optical period and $\tau_{T}$ the tunneling time (which is not strictly related to quantum mechanical tunneling time). From this expression, it is evident that when $\gamma \ll 1$, i.e., $\tau_{T} \ll T_{0}$, the Coulomb barrier is stationary during tunneling, while, when $\gamma \gg 1$, i.e., $\tau_{T} \gg T_{0}$, the barrier is oscillating. Thus, in general, when $\gamma \ll 1$, tunnel ionization dominates, while, when $\gamma \gg 1$, multiphoton ionization does. In reality, these two ionization regimes cannot be completely separated from each other: ${ }^{51,52}$ electrons show up in the continuum around the peaks of the driving electric field, $E(t)$, but not only for $\gamma<1$, also for $\gamma>1$.

The ionization rate can be calculated by employing the Perelomov, Popov and Terent'ev (PPT) model for both tunnel and multiphoton ionization. ${ }^{53-55}$ In the tunnelling regime $(\gamma \ll 1)$ the probability of ionization as a function of the laser electric field can be calculated by using the simpler Ammosov, Delone and Krainov (ADK) model. ${ }^{56}$ Assuming that most of the tunnel ionization is confined to a very small portion of an optical cycle, so that the driving electric field, $E$, can be considered as quasi-static, the tunnel ionization rate is given by the following expression (in atomic units):

$$
w=-\frac{1}{N} \frac{d N}{d t}=I_{p}\left|C_{n^{*} \ell^{*}}\right|^{2} G_{\ell m}\left(\frac{2 F_{0}}{E}\right)^{2 n^{*}-|m|-1} \exp \left(-\frac{2 F_{0}}{3 E}\right)
$$


where $N$ is the ground-state population,

$$
\begin{aligned}
& \left|C_{n^{*} \ell^{*}}\right|^{2}=\frac{2^{2 n^{*}}}{n^{*} \Gamma\left(n^{*}+\ell^{*}+1\right) \Gamma\left(n^{*}-\ell^{*}\right)}, \\
& G_{\ell m}=\frac{(2 \ell+1)(\ell+|m|) !}{2^{|m|}|m| !(\ell-|m|) !}, \quad F_{0}=\left(2 I_{p}\right)^{3 / 2},
\end{aligned}
$$

$\Gamma$ is the Euler Gamma function, $n^{*}=\sqrt{I_{p H} / I_{p}}$ is the effective principal quantum number, $I_{p H}$ is the ionization potential of hydrogen, $\ell^{*}=n^{*}-1$ is the effective orbital quantum number, $n$ is the principal quantum number, $\ell$ the angular quantum number and $m$ the magnetic quantum number. Due to the exponential dependence on the external electric field, the ionization rate is strongly dependent on the driving intensity. This is particularly important since this highly nonlinear process directly determines the ultrafast (sub-femtosecond) temporal duration of the electronic wave packet generated after tunnel ionization, as will be discussed in the following.

\subsubsection{Motion of the electron in the driving field and recombination}

After tunnel ionization, the electron is free to move in the continuum under the effect of the electric field of the fundamental radiation. We can assume that the freed electron appears in the continuum with velocity $\mathbf{v}=0$. Assuming a monochromatic driving field, $E$, of amplitude $E_{0}$, angular frequency $\omega_{0}$, and linearly polarized in the $x$ direction defined by the unitary vector $\mathbf{u}_{x}$

$$
\mathbf{E}(t)=E_{0} \cos \left(\omega_{0} t\right) \mathbf{u}_{x}
$$

the equation of motion of the electron after tunnel ionization is

$$
\frac{d \mathbf{v}}{d t}=-\frac{e}{m} \mathbf{E}(t)
$$

where $m$ and $-e$ are the electron mass and charge, respectively (in the remaining part of this section we will not use atomic units for the sake of clarity). This equation of motion can be 
easily integrated, thus giving the velocity of the electron at any time $t$ after ionization:

$$
\mathbf{v}(t)=-\frac{e E_{0}}{m \omega_{0}}\left[\sin \left(\omega_{0} t\right)-\sin \left(\omega_{0} t^{\prime}\right)\right] \mathbf{u}_{x},
$$

where $t^{\prime}$ is the tunnel ionization instant. The position of the electron after ionization is therefore given by:

$$
x(t)=-\frac{e E_{0}}{m \omega_{0}^{2}}\left[\cos \left(\omega_{0} t\right)-\cos \left(\omega_{0} t^{\prime}\right)+\omega_{0}\left(t-t^{\prime}\right) \sin \left(\omega_{0} t^{\prime}\right)\right],
$$

where we have assumed that the initial position of the electron after ionization is $x\left(t^{\prime}\right)=0$. In the case of linear polarization, the electron can return to the parent ion and recombine to the ground state, with the emission of a photon. The recombination instant can be calculated by solving the equation $x(t)=0$, which does not have analytical solutions. It is possible to show that the freed electron can return to the parent ion if $0 \leq \omega_{0} t^{\prime} \leq 80^{\circ}$ or $180^{\circ} \leq \omega_{0} t^{\prime} \leq 260^{\circ}$. The numerical solution of the equation $x(t)=0$ can be fitted by the following expression: ${ }^{57}$

$$
\frac{t}{T_{0}}=\frac{1}{4}-\frac{3}{2 \pi} \sin ^{-1}\left(4 \frac{t^{\prime}}{T_{0}}-1\right) .
$$

The evolution of the recombination time, $t$, as a function of the ionization time, $t^{\prime}$, in a temporal window corresponding to a quarter of the optical period is displayed in Fig. 4. It is interesting to note that the recombination instant is uniquely determined by the ionization instant. The maximum time interval spent by the freed electron in the continuum before recombination is a complete optical cycle. This situation corresponds to an electron set free by tunnel ionization at $t^{\prime}=0$ (i.e., exactly in correspondence of the electric field crest). In this case the electron returns to the parent ion with zero velocity. Upon increasing $t^{\prime}$ the recombination time decreases and the electron returns to the parent ion with a kinetic energy given by

$$
E_{k}=\frac{1}{2} m v^{2}(t)=2 U_{p}\left[\sin \left(\omega_{0} t\right)-\sin \left(\omega_{0} t^{\prime}\right)\right]^{2},
$$




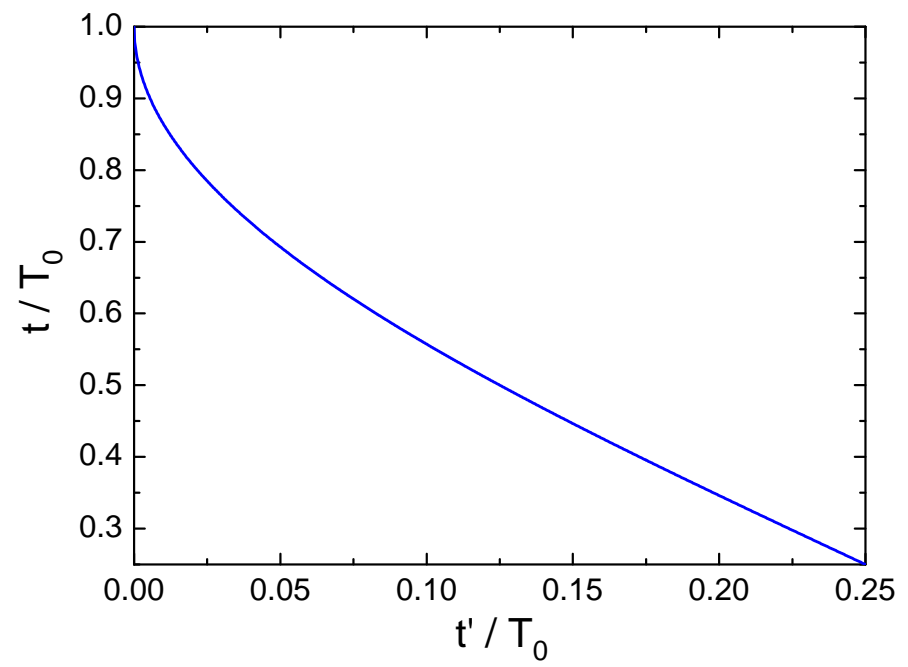

Figure 4: Recombination time normalized to the optical period $T_{0}, t / T_{0}$, as a function of the ionization time normalized to the optical period, $t^{\prime} / T_{0}$.

where $U_{p}=e^{2} E_{0}^{2} / 4 m \omega_{0}^{2}$ is the ponderomotive energy (the expression for $U_{p}$ has been already written earlier in this section in atomic units). Figure 5 displays the kinetic energy of the recolliding electron, normalized to the ponderomotive energy, as a function of the ionization instant. Upon increasing $t^{\prime}$ the kinetic energy increases up to a maximum value $E_{k} \approx 3.17 U_{p}$, which is carried by the electron emitted at $t^{\prime} / T_{0} \approx 0.05$ and returning to the parent ion at $t / T_{0} \approx 0.7$. Therefore, the maximum energy of the photons emitted by recombination of the returning electron is given by the following expression

$$
\hbar \omega_{\max }=I_{p}+3.17 U_{p}
$$

usually called the cutoff law. Therefore, a burst of high-energy photons, typically in the EUV spectral region, is generated upon recombination of the re-colliding electronic wave packet with the parent ion. Since $U_{p} \propto I_{0} \lambda_{0}^{2}$, where $I_{0}$ is the peak intensity of the driving pulse and $\lambda_{0}$ the corresponding wavelength, the cutoff frequency can be extended by increasing the intensity and the wavelength.

After the maximum at $t^{\prime} / T_{0} \approx 0.05$, the kinetic energy of the returning electron decreases and finally vanishes when $t^{\prime} / T_{0} \approx 0.25$. Figure 5 also shows that the electron released at a 


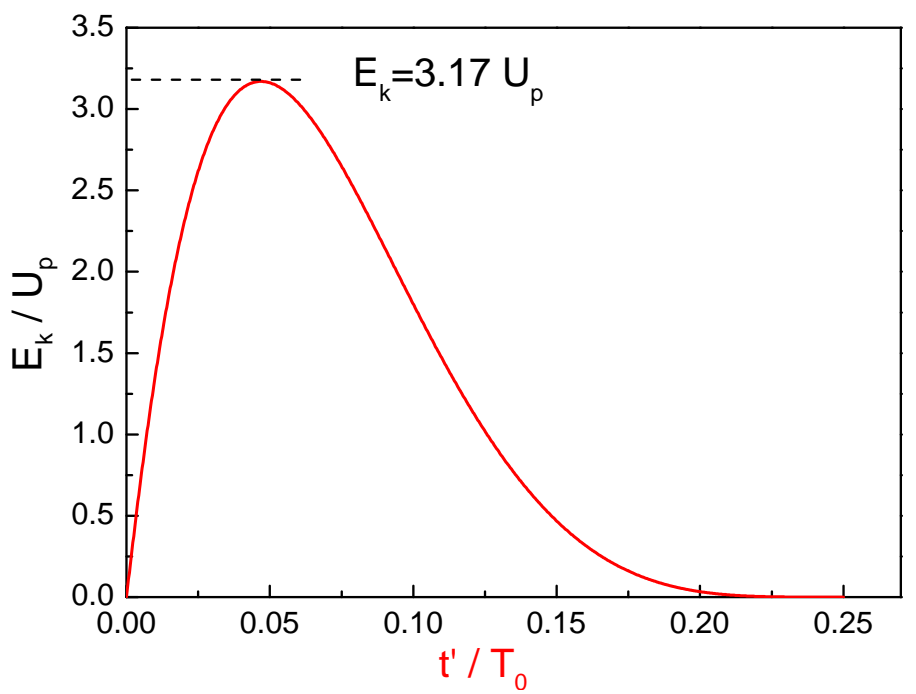

Figure 5: Evolution of the kinetic energy of the recolliding electron, normalized to the ponderomotive energy, as a function of the ionization time, $t^{\prime}$.

particular instant $t^{\prime} / T_{0}<0.05$ has the same kinetic energy of an electron emitted at $t^{\prime} / T_{0}>$ 0.05. Since shorter ionization times, $t^{\prime}$, correspond to longer recombination times, $t$ (see Fig. 4), it is common to group the trajectories followed by the electron between the tunneling and recombination times in two classes. The long trajectories correspond to electrons ionized at $t^{\prime} / T_{0}<0.05$, while the short trajectories correspond to electrons ionized at $t^{\prime} / T_{0}>0.05$. For short trajectories, the corresponding recombination time is $0.25<t / T_{0}<0.7$ and, for the long trajectories, $0.7<t / T_{0}<1$. The distinction between short and long trajectories can be clearly visualized by plotting the normalized kinetic energy of the recolliding electron as a function of both the ionization instant $t^{\prime}$ and the recombination instant $t$, as shown in Fig. 6. It is possible to select either the long or the short trajectories with a high degree of accuracy by simply changing the position of the gas target with respect to the focal point of the driving radiation and by using suitable spatial filters in the harmonic beam-path. Short and long trajectories give rise to EUV emission with very different characteristics, which are crucial for applications. In particular, since the kinetic energy of the re-colliding electronic wave packet changes with time, also the energy of the photons generated upon recombination changes with time: i.e, the generated EUV pulses posses an intrinsic chirp, $C=d \omega / d t$, named attochirp. The EUV pulses generated by the short trajectories exhibit a positive and almost 


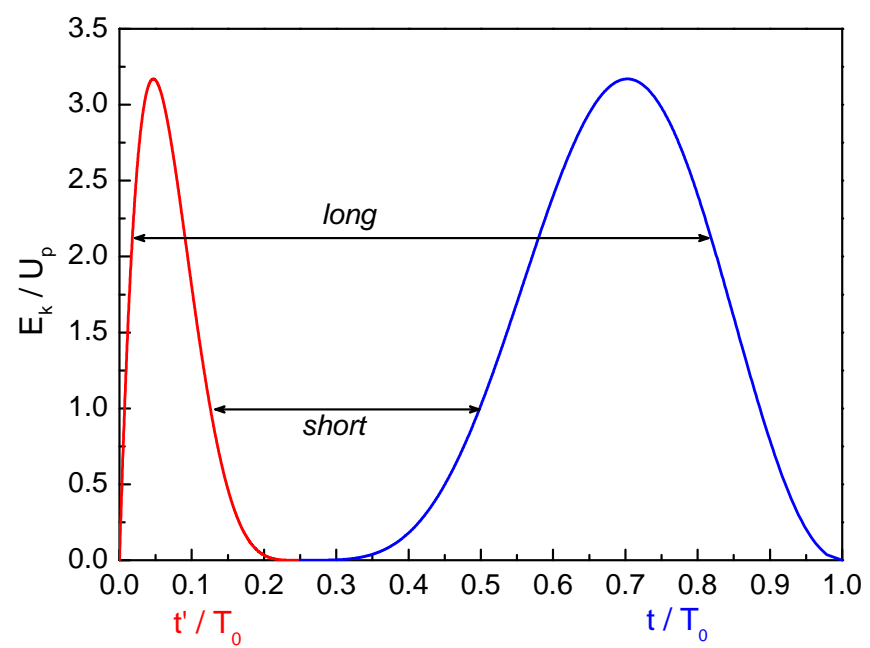

Figure 6: Evolution of the kinetic energy of the recolliding electron, normalized to the ponderomotive energy, as a function of the ionization time, $t^{\prime}$ (red curve) and of the recollision time, $t$ (blue curve). Short and long trajectory are displayed.

linear chirp, meaning that the instantaneous frequency increases with time, while the pulses generated by the long trajectories are negatively chirped.

Since the processes at the basis of the semiclassical three-step model (i.e., tunnel ionization, acceleration in the laser electric field and recombination) are repeated every half optical cycle of the driving radiation, a train of EUV pulses, separated by $T_{0} / 2$, is generated. In the spectral domain, this corresponds to the generation of high-order harmonics of the fundamental radiation. A rough estimate of the duration of the EUV pulses produced by HHG can be obtained by combining the results of the semiclassical three-step model with calculations of the tunnel ionization rate. In particular, it is possible to calculate an approximate value for the duration of the electronic wave packet generated by tunnel ionization, which in turn is related to the duration of the EUV pulse emitted upon recombination.

Let us consider harmonic generation in neon $\left(I_{p}=21.56 \mathrm{eV}, F_{0}=1.02 \times 10^{12} \mathrm{~N} / \mathrm{C}, n^{*}=0.79\right.$, $m=0)$ and assume that only the short trajectories are selected $\left(t^{\prime} / T_{0}>0.05\right)$. By choosing a driving intensity $I=1.4 \times 10^{13} \mathrm{~W} / \mathrm{cm}^{2}$, we have $F_{0} / E=100$, so that the ADK ionization 
rate can be written as

$$
w=\frac{I_{p}}{\hbar}\left|C_{n^{*} \ell^{*}}\right|^{2} G_{\ell m}\left(\frac{200}{\left|\cos \left(\omega t^{\prime}\right)\right|}\right)^{0.58} \exp \left(-\frac{200}{3\left|\cos \left(\omega t^{\prime}\right)\right|}\right)
$$

which vanishes already at $t^{\prime} / T_{0}=0.07$. Therefore, in the case considered in this example, the strongly nonlinear dependence of the ionization rate with the driving intensity determines a very short temporal window for tunnel ionization, in the range $0.05<t^{\prime} / T_{0}<0.07$. By

using the approximate formula (8), the limited window for ionization determines a short window for the recombination time, $0.63<t / T_{0}<0.7$, thus limiting to $\sim 180$ as the spread in recombination times: this number is directly related to the duration of the EUV pulse emitted upon recombination.

\subsection{Semiclassical formulation of the three-step model based on strong- field approximation}

A semiclassical formulation of the three-step model based on the strong-field approximation (SFA) was developed in 1994 by Lewenstein et al. ${ }^{58}$ In SFA, the electron in the continuum is treated as a free particle moving in the electric field, i.e. the influence of the Coulomb potential is completely neglected. In spite of this limitation, Lewenstein's model has been widely used to understand the HHG process in atoms and molecules. Subsequently, efforts have been made to improve the model by including Coulomb distortion.

High-order harmonic generation is the result of the macroscopic response of the medium:

$$
\frac{\partial^{2} E}{\partial z^{2}}-\frac{1}{c^{2}} \frac{\partial^{2} E}{\partial t^{2}}=\mu_{0} \frac{\partial^{2} P}{\partial t^{2}}
$$

where $P$ is the polarization of the medium and $\mu_{0} \partial^{2} P / \partial t^{2}$ represents the source term for the EUV field and it is due to the interaction with the medium. By definition, the polarization 
is the dipole moment per unit volume:

$$
\mathbf{P}(t)=N\langle e \mathbf{r}(t)\rangle
$$

where $\langle e \mathbf{r}(t)\rangle$ is the expectation value of the dipole moment of an atom in the time domain. Assuming atomic units, the dipole moment can be written as follows:

$$
\langle\mathbf{r}(t)\rangle=\langle\psi(\mathbf{r}, t)|\mathbf{r}| \psi(\mathbf{r}, t)\rangle
$$

It is evident that the first step to evaluate the response is the calculation of the wave function $|\psi(\mathbf{r}, t)\rangle$.

Considering a single atom in a classical electromagnetic field, the Schrödinger equation can be solved using the following three approximations: (i) single active electron (SAE) approximation; (ii) strong field approximation (SFA); (iii) in the calculations, only the ground state and the continuum are considered, i.e., the influence of the other bound states of the atom is completely neglected. In the framework of the SAE approximation the atom is treated as a hydrogen-like system and multiple ionization is neglected. Within this approximation, only one electron is active in the ionization process, while the other ones are involved only in the screening of the nucleus, thus giving rise to an effective single-electron potential. In the case of atoms ${ }^{59,60}$ and small molecules, ${ }^{61}$ the SAE approximation has been demonstrated to be a very useful approach. In polyatomic molecules with many delocalized electrons, however, the SAE approximation often fails to account for the experimental results. ${ }^{62,63}$ Indeed, in most molecules, multi-electron excitations typically lie below the first ionization potential and the inner valence electrons move with frequencies comparable to those for the outer valence electrons, thus rendering the SAE approximation inadequate. ${ }^{62}$

The time-dependent wave function of the system, $|\psi(\mathbf{r}, t)\rangle$, can be written as a superposition of the ground state wave function, $|g\rangle e^{i I_{p} t}$, and of the continuum wave function, which corresponds to the electron wave packet moving in the continuum after tunnel ionization. 
The source of HHG is given by the oscillating dipole moment generated by the interference of the continuum electron wave packet re-colliding with the parent ion with the fraction of the electron wave function left in the ground atomic state, as explained in Sect. 2.2 (see Fig. 3). For hydrogen-like atoms, the space-dependent part of the ground state $s$-wave function can be written as (from now on we use again atomic units):

$$
|g\rangle=\frac{\alpha^{3 / 4}}{\pi^{1 / 2}} e^{-\sqrt{\alpha}|\mathbf{r}|}
$$

An ansatz for $|\psi(\mathbf{r}, t)\rangle$ is given by

$$
|\psi(\mathbf{r}, t)\rangle=e^{i I_{p} t}\left[a(t)|g\rangle+\int d^{3} \mathbf{v} b(\mathbf{v}, t)|\mathbf{v}\rangle\right]
$$

where $a(t)$ is the amplitude of the ground state wave function and $b(\mathbf{v}, t)$ are the amplitudes of the continuum states. Ignoring the continuum-continuum contribution, $\left\langle\mathbf{v}|\mathbf{r}| \mathbf{v}^{\prime}\right\rangle$, to the dipole moment, the dipole moment responsible for the harmonic generation can be written as

$$
\langle\mathbf{r}(t)\rangle=\int d^{3} \mathbf{v} a^{*}(t) b(\mathbf{v}, t) \mathbf{d}^{*}(\mathbf{v})+\text { c.c. }
$$

where $\mathbf{d}^{*}(\mathbf{v})=\langle g|\mathbf{r}| \mathbf{v}\rangle$ is the dipole transition-matrix element from the continuum states to the ground state. The physical interpretation of eq 17 is the following: the origin of the harmonic radiation is the oscillating dipole generated by the recombination of the re-colliding electron wave packet $b(\mathbf{v}, t)$ with the ground state with amplitude $a(t)$. Using the SFA it is possible to obtain a simple expression for the dipole moment given by eq 14:

$$
\langle\mathbf{r}(t)\rangle=i \int_{0}^{t} d t^{\prime} \int d^{3} \mathbf{p} a^{*}(t) \mathbf{d}^{*}[\mathbf{p}+\mathbf{A}(t)] e^{-i S\left(\mathbf{p}, t, t^{\prime}\right)} a\left(t^{\prime}\right) \mathbf{E}\left(t^{\prime}\right) \mathbf{d}\left[\mathbf{p}+\mathbf{A}\left(t^{\prime}\right)\right],
$$

where $\mathbf{E}(t)$ is the electric field of the driving radiation and $\mathbf{A}(t)=-\partial \mathbf{E} / \partial t$ is the corresponding vector potential, $\mathbf{p}$ is the canonical momentum defined as $\mathbf{p}=\mathbf{v}(t)-\mathbf{A}(t), \mathbf{d}\left[\mathbf{p}+\mathbf{A}\left(t^{\prime}\right)\right]$ and $\mathbf{d}^{*}[\mathbf{p}+\mathbf{A}(t)]$ are the dipole transition moments between the ground state $\Psi_{g}$ and a con- 
tinuum state represented by a plane wave with kinetic momentum $\mathbf{p}+\mathbf{A}\left(t^{\prime}\right)$ and $\mathbf{p}+\mathbf{A}(t)$, respectively,

$$
\mathbf{d}[\mathbf{p}+\mathbf{A}(t)]=\langle\mathbf{p}+\mathbf{A}(t)|\hat{\mathbf{d}}| g\rangle
$$

and $S\left(\mathbf{p}, t, t^{\prime}\right)$ is the quasi-classical action, which gives the phase accumulated by the electron wave function during the propagation in the continuum:

$$
S\left(\mathbf{p}, t, t^{\prime}\right)=\int_{t^{\prime}}^{t} d t^{\prime \prime}\left\{\frac{\left[\mathbf{p}+\mathbf{A}\left(t^{\prime \prime}\right)\right]^{2}}{2}+I_{p}\right\}
$$

Equation 18 has a simple physical interpretation in complete agreement with the three-step quasi-classical model. The dipole moment is given by the contribution of all the electrons emitted in the continuum at an instant $t^{\prime}$ with momentum $\mathbf{p}$ with a probability given by the term $\mathbf{E}\left(t^{\prime}\right) \mathbf{d}\left[\mathbf{p}+\mathbf{A}\left(t^{\prime}\right)\right]$; the electron is then accelerated by the electric field and acquires a phase factor $e^{-i S\left(\mathbf{p}, t, t^{\prime}\right)}$, and finally recombines with the parent ion at an instant $t$ with probability $\mathbf{d}^{\star}[\mathbf{p}+\mathbf{A}(t)]$. In the spirit of Feynman's quantum paths approach, ${ }^{64}$ each electron trajectory has to be considered and integrations over the momentum space $d^{3} \mathbf{p}$ and over the ionization instants $d t^{\prime}$ are required. It is important to observe that the various electron trajectories (quantum paths) do not contribute in the same way to the electric dipole moment: due to the fast oscillating phase term, all the contributions tend to cancel out apart from those corresponding to the stationary points of the classical action:

$$
\nabla_{\mathbf{p}} S\left(\mathbf{p}, t, t^{\prime}\right)=0
$$

In this way the dipole moment can be written as a coherent superposition of only a few electron quantum paths, which are the complex trajectories followed by the electrons from the ionization instant to the recombination with the parent ion. Using the saddle-point method, the Fourier transform of the dipole moment, $x(\omega)$ (assuming a driving field polarized along the $x$-axis), can be written as a coherent superposition of the contributions from 
the different electron quantum paths corresponding to the complex saddle-point solutions $\left(\mathbf{p}_{s}, t_{s}, t_{s}^{\prime}\right)$, where $\mathbf{p}_{s}$ is the stationary value of the momentum acquired by an electron that is set free at time $t_{s}^{\prime}$ and recombines with the parent ion at time $t_{s}$. The sum over the relevant quantum paths can be decomposed in two terms related to the short and long quantum paths. The Fourier transform of the dipole moment can thus be written as ${ }^{65}$

$$
x(\omega)=\sum_{s \in \text { short }}\left|x_{s}(\omega)\right| \exp \left[i \Phi_{s}(\omega)\right]+\sum_{s \in \text { long }}\left|x_{s}(\omega)\right| \exp \left[i \Phi_{s}(\omega)\right]
$$

where the first sum takes into account the contributions of the short quantum paths, while the second one considers the long quantum paths. $\Phi_{s}(\omega)$ is the phase of the complex function $x_{s}(\omega)$.

\subsection{Generation of attosecond pulses}

In 1992 Farkas and Tóth proposed, for the first time, the possibility to use HHG in gases to generate attosecond pulses. ${ }^{66}$ Based on experimental and theoretical results available at that time, they predicted the generation of trains of $\sim 30-70$ as pulses separated by half optical cycle of the fundamental radiation. The intensity profile of the EUV pulses, $I(t)$, can be obtained if the spectral amplitudes, $A_{q}$, and the spectral phases, $\phi\left(\omega_{q}\right)$, of the generated $N$ harmonics are known:

$$
I(t)=\left|\sum_{q=1}^{N} A_{q} \exp \left[-i \omega_{q} t+i \phi\left(\omega_{q}\right)\right]\right|^{2},
$$

where $q$ is the harmonic order. The shortest pulse duration, corresponding to Fourier-limited pulses, is obtained when a linear relationship $\phi\left(\omega_{q}\right)=q \omega_{0} t_{e}$ holds, with an emission time, $t_{e}$, independent of the harmonic order. Indeed, in this case all harmonics are emitted at the same time, $t_{e}=\partial \phi / \partial \omega=\Delta \phi / 2 \omega_{0}$, where $\Delta \phi$ is the spectral phase between two consecutive harmonics (separated by $\left.2 \omega_{0}\right)$.

The first experimental proof of the generation of trains of attosecond pulses was reported by Paul et al. in 2001. ${ }^{6}$ By using a novel experimental technique, the Reconstruction of At- 
tosecond Beating By Interference of Two-photon Transitions (RABBITT), which can be seen as the first example of an attosecond measurement method, a near-linear phase relationship was measured among five consecutive harmonics generated in argon, corresponding to a train of 250-as pulses. The RABBITT method will be described in Sect. 3.1.1. The importance of the intrinsic chirp of the attosecond pulses was experimentally recognized by Mairesse et al. in $2003^{67}$. In the previous section we have pointed out that an important result of the three-step model is that the harmonic emission time $t_{e}$ varies quasi-linearly with frequency, thus leading to a quasi-linear chirp of the generated attosecond pulses, positive for the short trajectories and negative for the long trajectories. Unless this chirp is compensated for, the minimum pulse duration cannot be achieved by simply increasing the spectral bandwidth of the EUV pulses. In a first set of measurements, an attosecond pulse train was generated in argon at a driving intensity of $1.2 \times 10^{14} \mathrm{~W} / \mathrm{cm}^{2}$; short electron trajectories were selected by placing the gas jet after the focal point of the fundamental beam. ${ }^{68,69}$ In this case, the measured time shift between the emission of two consecutive harmonics, $q$ and $q+2$, defined as $\Delta t_{e}\left(\omega_{q+1}\right)=t_{e}\left(\omega_{q+2}\right)-t_{e}\left(\omega_{q}\right)$, was $\Delta t_{e}=106 \pm 8$ as, in good agreement with the results of the semiclassical single-atom model $\Delta t_{e}^{t h}=81 \pm 3$ as. It was observed, again in agreement with the theoretical model, that the synchronization in the harmonic emission improved upon increasing the driving intensity. Best synchronization was achieved in harmonic generation from neon. At a driving intensity of $3.8 \times 10^{14} \mathrm{~W} / \mathrm{cm}^{2}$, a nearly-linear variation of $t_{e}$ vs harmonic order was measured with $\Delta t_{e}=33 \pm 3$ as (close to the theoretical prediction $\Delta t_{e}^{t h}=26.1 \pm 0.2$ as), as shown in Fig. 7A . By measuring the spectral amplitudes and phases of the generated harmonics, it was possible to reconstruct the temporal intensity profile of the EUV pulses. The measurements clearly showed that the low harmonics are emitted close to the maxima of the driving electric field, while the highest harmonics are emitted close to the zero of the field (see Fig. 7B).

Even shorter pulses can be generated upon compensation of the intrinsic chirp. Positively chirped pulses can be temporally compressed close to the Fourier limit by introducing a 

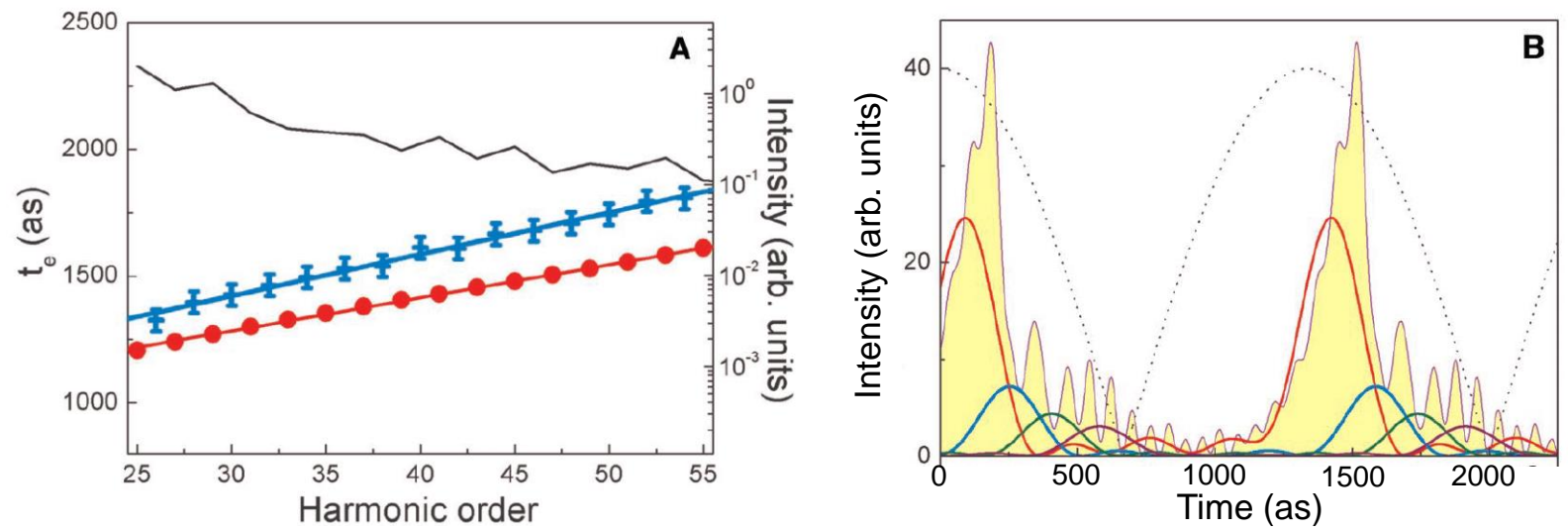

Figure 7: Harmonic generation in neon at a driving intensity of $3.8 \times 10^{14} \mathrm{~W} / \mathrm{cm}^{2}$. (A) Harmonic emission time vs harmonic order: measurements are represented by the blue crosses, the theoretical results by red dots. The black line is the measured harmonic intensity. (B) Temporal profile of harmonic emission corresponding to harmonics in the following five ranges: from 25th to 69th (yellow filled curve), from 25th to 33rd (red line), from 35th to 43rd (green line), from 45th to 53rd (blue line), and from 55th to 63rd (purple line). Black dots represent the absolute value of the laser electric field. Reproduced and adapted with permission from ref. 67. Copyright 2003 American Association for the Advancement of Science (AAAS).

system with a negative chirp. This problem has been successfully addressed by exploiting the negative dispersion of thin metallic filters. ${ }^{70,71}$ Negative dispersion is provided by aluminum filters in the energy range from 20 to $60 \mathrm{eV}$, by silicon filters in the range from 25 to 90 $\mathrm{eV}$ and by zirconium filters in the range from 70 to $160 \mathrm{eV}$. Another method that can be employed to compress broadband chirped EUV attosecond pulses has been proposed by using the influence on the pulse phase of a double-grating compressor at grazing incidence. ${ }^{72}$ The design of the attosecond compressor originates from the scheme of an EUV time-delay compensated monochromator designed to select a suitable portion of the harmonic spectrum without altering the femtosecond pulse time duration. ${ }^{73}$ It is able to introduce either a positive or negative dispersion in a broad band and can be operated in any spectral interval within the EUV and soft X-ray regions (10-300 eV). 


\subsubsection{Attosecond pulses in the water-window region}

For many applications, it is important to use attosecond pulses with high photon energy. In particular, generation of pulses in the water window is particularly important for the investigation of biological processes. Indeed, in this spectral region, ranging from the K-shell absorption edges of carbon $(284.2 \mathrm{eV}$, corresponding to $\sim 4.4 \mathrm{~nm})$ to the K-edge of oxygen (543.1 eV, corresponding to $~ 2.3 \mathrm{~nm})$, the carbon, oxygen and nitrogen atoms of a cell tissue show a high absorption, while their natural water environment is highly transparent. Even higher photon energies are required for the investigation of ultrafast electronic processes in correlated-electron, magnetic, and catalytic materials (Fe, Co, Ni, Cu), whose inner-shell absorption edges lie at photon energies nearing $1 \mathrm{keV} .{ }^{74,75}$ As already pointed out, a very effective way to increase the harmonic photon energy is to increase the driving wavelength. Since the first experimental demonstration of cutoff extension in HHG by using infrared (IR) pulses at $1.51 \mu \mathrm{m},{ }^{76}$ many groups have developed mid-IR sources for HHG. For exam-

ple, high-energy optical parametric amplifiers (OPAs) have been developed for HHG. ${ }^{77-80}$ The main drawback of long-wavelength driving radiation is related to the spatial spreading experienced by the wave packet of the re-colliding electron between tunnel ionization and recombination with the parent ion. This effect leads to a smaller recombination probability and to a lower conversion efficiency. Indeed, it has been experimentally demonstrated that the harmonic yield at constant laser intensity scales as $\lambda^{-6.3 \pm 1.1}$ in xenon and as $\lambda^{-6.5 \pm 1.1}$ in krypton over the driving wavelength range of $800-1850 \mathrm{~nm} .{ }^{81}$

A remarkable step forward to overcome the unfavourable scaling law of harmonic yield with the driving wavelength has been reported in $2012^{82}$ with the generation of bright EUV supercontinua covering photon energies from the extreme ultraviolet up to $1.6 \mathrm{keV}$, in principle allowing the generation of EUV pulses as short as 2.5 attoseconds. This result was obtained by focusing 3.9- $\mu \mathrm{m}$ wavelength pulses into a hollow-core fiber filled with He gas at very high pressure. The capability of this driving source to generate isolated attosecond pulses in the keV spectral region has been discussed in Ref. 83. Recently, few-optical-cycle 
IR pulses have been employed for the generation of high-photon energy harmonics. Using sub-two-cycle optical pulses at $1.6 \mu \mathrm{m}$ with stable carrier-envelope phase (see next paragraph), HHG in the water window was demonstrated. ${ }^{84}$ X-ray absorption measurements at the carbon K-edge from a high-flux water-window source providing radiation up to $400 \mathrm{eV}$ based on HHG with a 1-kHz, sub-2-cycle source at $1.85 \mu \mathrm{m}$ with CEP stability was reported in 2014. ${ }^{85}$ With the same laser source Silva et al. have recently demonstrated experimentally the isolation of individual attosecond pulses at the carbon K-shell edge with pulse duration below 400 as and with a bandwidth supporting a 30 -as pulse duration. ${ }^{86}$

\subsubsection{EUV pulses with circular polarization}

Another quite important development in attosecond technology is the generation of ultrashort EUV pulses with circular polarization. Circularly polarized EUV pulses can be used for the analysis of the structural, electronic and magnetic properties of matter using various experimental techniques: e.g., photoelectron circular dichroism for the investigation of chiral molecules, ${ }^{87}$ angle-resolved photoemission spectroscopy (ARPES) with circularly polarized pulses $^{88}$ and X-ray magnetic circular dichroism (XMCD) spectroscopy for the investigation of magnetic materials. ${ }^{89}$ Synchrotron radiation is typically used for these applications. Tabletop sources of pulses in the EUV with circular polarization and ultrashort duration (from tens of femtoseconds to hundreds of attoseconds) would produce an enormous boost for the investigation of ultrafast processes involved in chirality-sensitive light-matter interactions. Various techniques have been proposed and implemented for the generation of high-order harmonics with circular polarization. ${ }^{90}$ Elliptically polarized harmonics with relatively small ellipticity $(\epsilon<0.37)$ have been generated by using elliptically polarized pulses, ${ }^{91}$ with the drawback of a strong decrease of harmonic yield with increasing ellipticity of the driving

radiation. A different experimental approach is based on harmonic generation from aligned molecules driven by linearly polarized pulses. ${ }^{92} \mathrm{~A}$ circular polarizer operating in reflection has been developed based on four mirrors coated with $35 \mathrm{~nm}$ of molybdenum and a $5-\mathrm{nm}$ 
layer of $\mathrm{B}_{4} \mathrm{C} .{ }^{93}$ Harmonics with almost perfect circular polarization were obtained in the spectral range between 18.1 and $26.3 \mathrm{~nm}$, with an efficiency between 2.6 and $4.4 \%$. The main disadvantages are the high losses and the limitation in spectral bandwidth imposed by the available multilayer mirror materials.

A different approach was reported in $1995^{94,95}$ based on the use of two circularly polarized, counter-rotating fields at the fundamental and second harmonic wavelength. The same technique was employed in 2014 by Fleischer et al., who reported on a full control over the polarization of the harmonics, from circular through elliptical to linear polarization, without deterioration of the harmonic conversion efficiency. ${ }^{96}$ By employing phase-matching in a gas-filled waveguide, bright circularly polarized harmonics were generated with this technique by Kfir et al.. ${ }^{97}$ Another technique, recently demonstrated by Ferré et al., is based on resonant harmonic generation with elliptically polarized driving pulses. ${ }^{98}$ In 2013 an interesting method for the generation of isolated attosecond pulses with circular polarization was reported, ${ }^{99}$ based on $\mathrm{HHG}$ in a molecular medium driven by an intense elliptically polarized laser pulse in the presence of an intense terahertz field. A possible application of these pulses, proposed by Yuan and Bandrauk, is the generation of intense attosecondmagnetic-field pulses in molecules. ${ }^{100}$

\subsection{Isolated attosecond pulses}

\subsubsection{Brief overview of femtosecond laser technology}

So far we have reported on the generation of trains of attosecond pulses, but if the harmonic generation process is confined to a single event, it is possible to produce isolated attosecond pulses. Immediately after the first demonstration of attosecond pulse trains by Paul et al., ${ }^{6}$ the generation of isolated attosecond pulses, with a duration of about 650 as, was reported in the same year by Hentschel et al.. ${ }^{7}$ A few developments in femtosecond laser technology were crucial for reliable generation of isolated attosecond pulses, in particular the stabilization of the carrier-envelope phase (CEP) of the driving pulses and the generation of high-peak- 
power, few-optical-cycle pulses.

The CEP of a pulse, whose electric field can be written as $E(t)=E_{0}(t) \cos \left(\omega_{0} t+\phi\right)$, is the phase offset, $\phi$, between the maximum of the pulse envelope, $E_{0}(t)$, and the maximum of the carrier wave at frequency $\omega_{0}$. In a typical laser oscillator, the CEP changes from pulse to pulse. Indeed, each time the pulse travels in the cavity through the active medium, a mismatch between the group velocity and the phase velocity occurs, so that a systematic pulse-to-pulse CEP slippage, $\Delta \phi$, is present at the output of the cavity. Moreover, $\Delta \phi$ is not constant since, for example, energy variations of the pump laser translate into additional CEP fluctuations by means of the nonlinear Kerr effect inside the active laser material. The self-referencing phase stabilization technique, ${ }^{101-104}$ first introduced for frequency metrology, has revolutionized the field of attosecond physics. CEP stabilization can be achieved by using active or passive methods: in the first case, electronic feedback loops are required, while, in the second case, CEP fluctuations are automatically cancelled by all-optical methods. In general, active stabilization of a Ti:sapphire laser system based on chirped-pulse amplification (CPA $)^{105}$ is obtained by using two feedback loops: a fast loop for CEP stabilization at the output of the laser oscillator and a slow-loop to compensate for residual CEP variations introduced by the amplification process. ${ }^{106}$ Passive CEP stabilization was pioneered by Baltuška et al. ${ }^{107}$ and it is based on the process of difference-frequency generation (DFG) in a second order nonlinear crystal. The first experimental evidence of the CEP role of fewcycle pulses has been obtained in strong-field photoionization, using 6-fs pulses with random CEP. 108

The laser systems used for the generation of attosecond pulses are typically based on CPA Ti:sapphire lasers, which routinely deliver 20-fs, 800-nm, CEP-stabilized pulses at the multi-millijoule level. A promising alternative for the generation of ultrashort laser pulses is offered by the optical parametric chirped pulse amplification (OPCPA) technique, ${ }^{109}$ which combines optical parametric amplification and CPA to obtain few-optical-cycle pulses with peak power up to the petawatt level. As discussed in a review on recent advances in 
femtosecond technology, ${ }^{110}$ OPCPA driven by terawatt-scale pulses from ytterbium lasers at kilowatt-scale average power will be the basis for third-generation femtosecond technology, which will combine high (terawatt-scale) peak powers with high (kilowatt-scale) average powers in ultrashort optical pulse generation.

Another route for the generation of few-optical-cycle pulses is the implementation of post-compression techniques. In order to improve the photon flux of the attosecond sources, efforts have been made to increase the energy of the broadband driving pulses and different approaches for post-compression have been developed. The most common technique for pulse compression of high-energy femtosecond pulses is based on propagation in a gas-filled hollow-core fiber (HCF) in combination with ultrabroadband dispersion compensation. ${ }^{111-113}$ Nowadays, sub-5 fs pulses with a few millijoules energy obtained by using this compression technique are available in many laboratories. To scale the hollow-fiber technique to higher peak intensities $\left(>10^{15} \mathrm{~W} / \mathrm{cm}^{2}\right)$ it is possible to employ spectral broadening induced by gas ionization in an HCF filled with helium at low gas pressure: output pulse energy of $13.7 \mathrm{~mJ}$ and pulse duration of $11.4 \mathrm{fs}$ were obtained. ${ }^{114}$

As mentioned above, besides the CEP stabilization, it is also important for various applications to increase the repetition rate of the driving pulses up to the megahertz level, ${ }^{115}$ for example, in the case of coincidence measurement of charged particles, which typically requires less than one event per laser shot in order to uniquely assign to the same reaction the measured particles. Surface science and condensed matter studies often suffer from space charge effects, which could be mitigated by reducing the number of photons per pulse with a corresponding increase in the pulse repetition rate to increase the signal-to-noise ratio. Several techniques for HHG at high repetition rates have been experimentally investigated over the past years. ${ }^{26} \mathrm{~A}$ very promising scheme is based on the use of passive enhancement cavities, where a coherent buildup of pulses delivered at the $\mathrm{MHz}$ repetition rate by a laser cavity is achieved in order to reach the intensity required for efficient harmonic generation. ${ }^{116-119}$ Another technique employs femtosecond fiber-based laser systems, which can generate aver- 
age output powers of nearly $1 \mathrm{~kW},{ }^{120}$ pulse energies sufficient for $\mathrm{HHG}$ at repetition rates as high as a few MHz. As recently reported by Hädrich et al., a broad plateau (ranging from $25 \mathrm{eV}$ to $40 \mathrm{eV}$ ) of strong harmonics, each containing more than $1 \times 10^{12}$ photons $/ \mathrm{s}$, has been generated by achieving phase-matched HHG of a MHz fiber laser. ${ }^{121}$ High-average power Yb-fiber laser systems have been also used to pump OPCPA systems at up to $1 \mathrm{MHz}$ repetition rate, ${ }^{122}$ which have been used for the generation of isolated attosecond pulses. ${ }^{123}$

\subsubsection{Generation of isolated attosecond pulses}

By taking advantage from these impressive technical developments in ultrafast laser technology, various schemes have been introduced to confine the harmonic generation to a single

event. In general, the production of isolated pulses requires the generation of a gating of the harmonic radiation or of the harmonic generation process. Although a few schemes are based on the combination of different gating mechanisms, the principles of operation can be divided in spectral, temporal and spatial gating. Only a brief description of the main gating techniques will be reported here, since this topic is already covered by various reviews. ${ }^{90,124-126}$

The first isolated attosecond pulses were generated by employing the amplitude gating technique, based on the spectral selection of the cutoff portion of the harmonic radiation produced by few-optical-cycle driving pulses without CEP stabilization. ${ }^{7} \mathrm{~A}$ few years later, the same authors used CEP stable, 5-fs fundamental pulses to generate 250-as EUV pulses with the same technique. ${ }^{127}$ The idea, first proposed by Christov et al., ${ }^{128}$ is to select the attosecond pulse generated by the most intense half-cycle of the fundamental pulse, which corresponds to the cutoff portion of the EUV spectrum.

The temporal gating schemes are based on the production of an ultrashort window, where harmonic generation is allowed. To generate isolated pulses, the temporal duration of this gate must be shorter than the separation between two consecutive attosecond pulses (half- 
optical cycle or a complete optical cycle in the case of two-colour excitation). The most common temporal gating schemes are the following: polarization gating, double optical gating and ionization gating. The first two techniques are based on the strong dependence of the harmonic generation process on the polarization of the driving radiation. Indeed, as a direct consequence of the physical processes leading to HHG, the generation yield strongly decreases upon increasing the ellipticity of the driving radiation: in the case of elliptical or circular polarization, the electron wavepacket produced by tunnel ionization cannot return to the parent ion, thus leading to a negligible harmonic generation. Measurements performed by Burnett et al. showed that the harmonic efficiency is reduced by a factor of $\sim 2$ for an ellipticity $\varepsilon_{t h}$ of $0.13 .{ }^{129}$ In 1994 Corkum et al. proposed to generate sub-femtosecond pulses by using a driving pulse with a time-dependent polarization state: ${ }^{130}$ circular on the leading and trailing edges and almost linear just around the peak of the pulse, where efficient harmonic generation would be confined. The proposed method for the generation of such time-dependent polarization state was based on the combination of two perpendicularly polarized pulses with frequencies $\omega_{1}$ and $\omega_{2}$ slightly detuned $\left(\left(\omega_{1}-\omega_{2}\right) \ll\left(\omega_{1}+\omega_{2}\right) / 2\right)$. Due to the coherent superposition of the two fields, the polarization state changes through the pulse being circularly polarized on the edges and linearly polarized at the center. Tcherbakoff et al. proposed a different and very simple setup based on the use of single-color driving pulses in combination with two birefringent plates. ${ }^{131}$ This method was experimentally demonstrated in 2006 first by spectral measurements ${ }^{132}$ and then by measuring the duration of the produced isolated attosecond pulses. ${ }^{133}$ By using a two-color excitation obtained by adding the second harmonic to the fundamental frequency (double-optical gating, DOG and generalized DOG, GDOG) it is possible to increase the temporal separation between two consecutive pulses from half optical cycle to a complete optical cycle of the fundamental radiation, thus relaxing the requirements on the duration of the driving pulse. ${ }^{134-138}$

Another temporal gating scheme is based on the use of the laser-induced ionization of the generating medium (ionization gating, IG). If the driving field is intense enough the 
plasma density rapidly increases on the leading edge of the laser driving pulse, thus creating a phase mismatch responsible for the suppression of HHG for all later half-cycles. Even if the intensity of the driving field is not enough to create a temporal gate as narrow as required to select a single attosecond pulse, the IG can be used in combination with bandpass filtering to overcome this problem. ${ }^{139,140}$ In 2010 another approach based on the combined action of complete population depletion and spatial filtering of the EUV beam has been employed to obtain an efficient temporal gating on the HHG process. ${ }^{141}$ Isolated attosecond pulses with time duration down to 155 as and an energy on target of a few nanojoules have been generated using this method.

A different approach for the generation of isolated pulses is based on the use of the rotation of the wavefront of the driving laser to generate a train of attosecond pulses, where each pulse is emitted in a slightly different direction corresponding to the instantaneous propagation direction of the excitation field at the instant of generation. This technique, called attosecond lighthouse and first proposed by Vincenti and Quéré, ${ }^{142}$ can be considered a spatial gating method, since the selection of a single pulse is obtained by spatially filtering the EUV radiation. The experimental test of this technique was reported in 2012 in the case of EUV harmonics produced from a plasma mirror ${ }^{143}$ and in 2013 in the case of gas harmonics. ${ }^{144}$ More recently the isolated attosecond pulses generated by using the lighthouse method have been temporally characterized. ${ }^{145}$ A noncollinear optical gating (NOG) technique, similar to the attosecond lighthouse approach, has been recently demonstrated. ${ }^{146}$ This gating scheme is based on the noncollinear superposition of two identical laser pulses at the position of the focus in the gas jet. This superposition results in a transverse intensity grating with a periodicity depending on both the carrier wavelength and the noncollinear angle. At zero time delay between the two replicas, the EUV radiation is generated along the bisector angle of the two fundamental beams. On the other hand, if a small delay $\Delta t$ is introduced, the EUV pulses originating from consecutive half-cycles are angularly separated from each other. Thus, each attosecond pulse can be isolated by a spatial selection in the far field. NOG is a 
particularly promising gating technique and outcoupling method for intracavity HHG.

Coherent synthesis of pulses is another way to produce sub-femtosecond pulses. ${ }^{147-149}$ An ultrabroadband continuum, extending from 1.1 to $4.6 \mathrm{eV}$, was generated in an hollow fiber filled with neon. The beam at the output of the fiber was divided into four beams with broad spectral bandwidths centred in the near infrared (about 1.1-1.75 eV), visible (about 1.75-2.5 eV), visible-ultraviolet (about 2.5-3.5 eV) and deep ultraviolet (about 3.5-4.6 eV). The pulses in the four arms of an interferometer were subsequently individually compressed by proper chirped mirrors, and then spatially and temporally overlapped to generate single pulses characterized by an ultrabroad spectrum. Pulses as short as 380 as were recently measured. ${ }^{149}$

\section{Attosecond experimental techniques}

\subsection{Attosecond metrology}

As a general rule, the use of ultrafast laser technology for time-resolved spectroscopic studies of matter requires the development of ultrafast metrology. Similarly, attosecond technology requires to temporally characterize attosecond pulses. The most widely used techniques can be divided in two categories, which can be classified according to whether the measurement is performed in a different medium or in the same medium used for the generation. In the first approach, called ex-situ, the photoelectrons produced in the medium by the attosecond pulse are perturbed by the presence of a synchronized laser field (top panel of Fig. 8), while, in the second approach, called in-situ, the electron trajectory is gently perturbed during the attosecond pulse generation process itself (bottom panel of Fig. 8).

\subsubsection{RABBITT}

Among the ex-situ techniques, the Reconstruction of Attosecond Beating By Interference of Two-photon Transitions (RABBITT) was the first method proposed and implemented for 


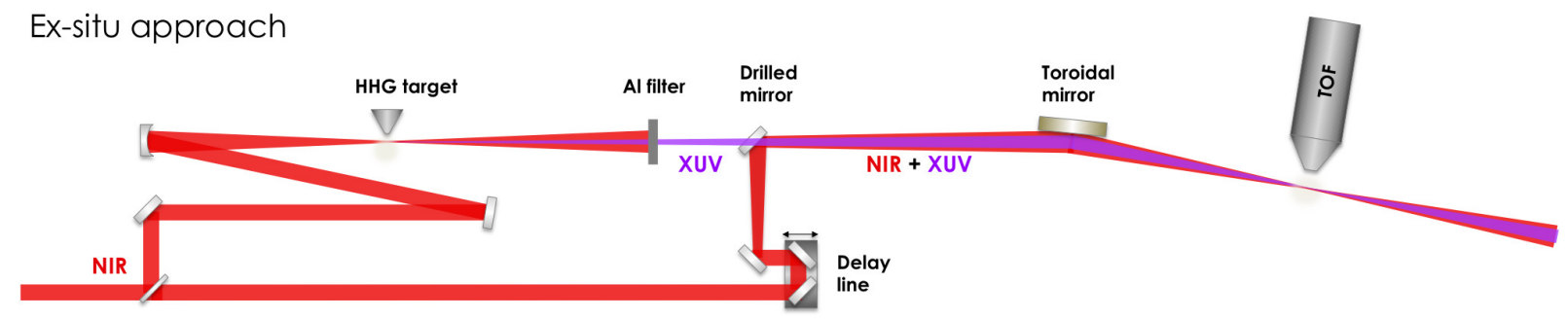

In-situ approach

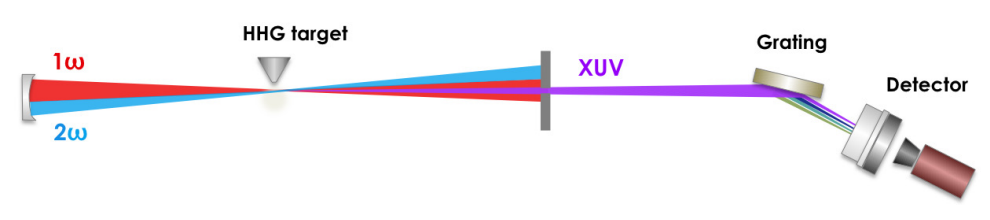

Figure 8: Top panel: scheme of the ex-situ approach. EUV attosecond pulses are generated in a first gas jet using a portion of the NIR laser beam. The remaining part of the laser beam is recombined with the EUV beam in order to be collinearly focused (with variable time delay) in a second gas jet where the measurement is performed. TOF: time of flight spectrometer. Bottom panel: scheme of the in-situ approach. A fundamental laser beam is focused together with its second harmonic beam (perturbing beam) in a gas jet to produce the EUV radiation. The high-order harmonic spectrum is then detected by a conventional EUV spectrometer.

the temporal characterization of trains of attosecond pulses. ${ }^{6,67,71}$ In this method, the photoelectrons produced by ionization of a target gas by the attosecond pulses are measured in the presence of a weak and time-delayed IR laser field. As illustrated in Fig. 9, the photoelectron spectrum produced by the EUV pulse train is a replica of the harmonic spectrum with discrete peaks separated by $2 \hbar \omega$, where $\omega$ is the frequency of the driving field in the HHG process. When a synchronized IR field is added, additional peaks at $\pm \hbar \omega_{I R}$ with respect to the peaks generated by the EUV harmonics are produced in the photoelectron spectrum. These additional peaks appear as sidebands of the peaks generated by the harmonics and are due to the absorption or emission of one (or more) IR photons. In the case of weak IR intensities, only one IR photon can be absorbed or emitted, so that each harmonic has only a single sideband on each side. Two different paths can contribute to the generation of the same sideband: absorption of one EUV photon corresponding to harmonic $q+1$ and emission 
of one IR photon or absorption of one EUV photon corresponding to harmonic $q-1$ and absorption of one IR photon. As the two paths are indistinguishable, they interfere and, as a result of this, the amplitude of the sidebands, SB, exhibits a periodic modulation ${ }^{150,151}$

$$
\mathrm{SB}=A_{f} \cos \left(2 \omega \tau-\Delta \phi_{q}-\Delta \phi_{\text {atom }}^{f}\right)
$$

where $A_{f}$ depends on the matrix dipole moments between the initial and final states, $\tau$ is the delay between the EUV and the IR pulses, $\Delta \phi_{q}=\phi_{q+1}-\phi_{q-1}$ is the phase difference between the harmonics $q+1$ and $q-1$, and $\Delta \phi_{\text {atom }}^{f}$ is the intrinsic phase difference of the matrix elements corresponding to photoionization from the $q+1$ and $q-1$ harmonics. Equation 24 clearly indicates that upon changing the delay $\tau$, the sideband amplitude oscillates at twice the frequency of the IR field (see right panel of Fig. 9). Since, for a suitable choice of the target gas, the intrinsic phase $\Delta \phi_{\text {atom }}^{f}$ can be precisely calculated, the phase difference between consecutive harmonics can be extracted from the time-delay scan over the entire harmonic spectrum, thus allowing a complete characterization of the attosecond pulse train. As mentioned in Sec. 2.4, this technique was successfully implemented for the first time by Paul et al in 2001. ${ }^{6}$ A 40 -fs $800-n m$ laser was split in two arms: a portion of the beam was used to generate the harmonics in an argon gas jet, while the remaining part of the IR beam was focused together with the EUV beam in a second argon gas jet, producing photoionization. The resulting photoelectron spectrum was then analyzed in a time-of-flight (TOF) electron spectrometer as a function of the relative delay between the two pulses. From the time dependent measurement a quasi-linear phase was extracted and a pulse duration of each individual pulse of the train was estimated to be 250 as. The limited number of harmonics analyzed in this first measurement (from 11 to 19) did not allow the intrinsic chirp of the attosecond pulse (atto chirp) being revealed. As mentioned above, a subsequent observation over a larger number of harmonics by Mairesse et al ${ }^{67}$ confirmed the presence of a quadratic phase, which has been assigned to the lack of synchronization in the emission 


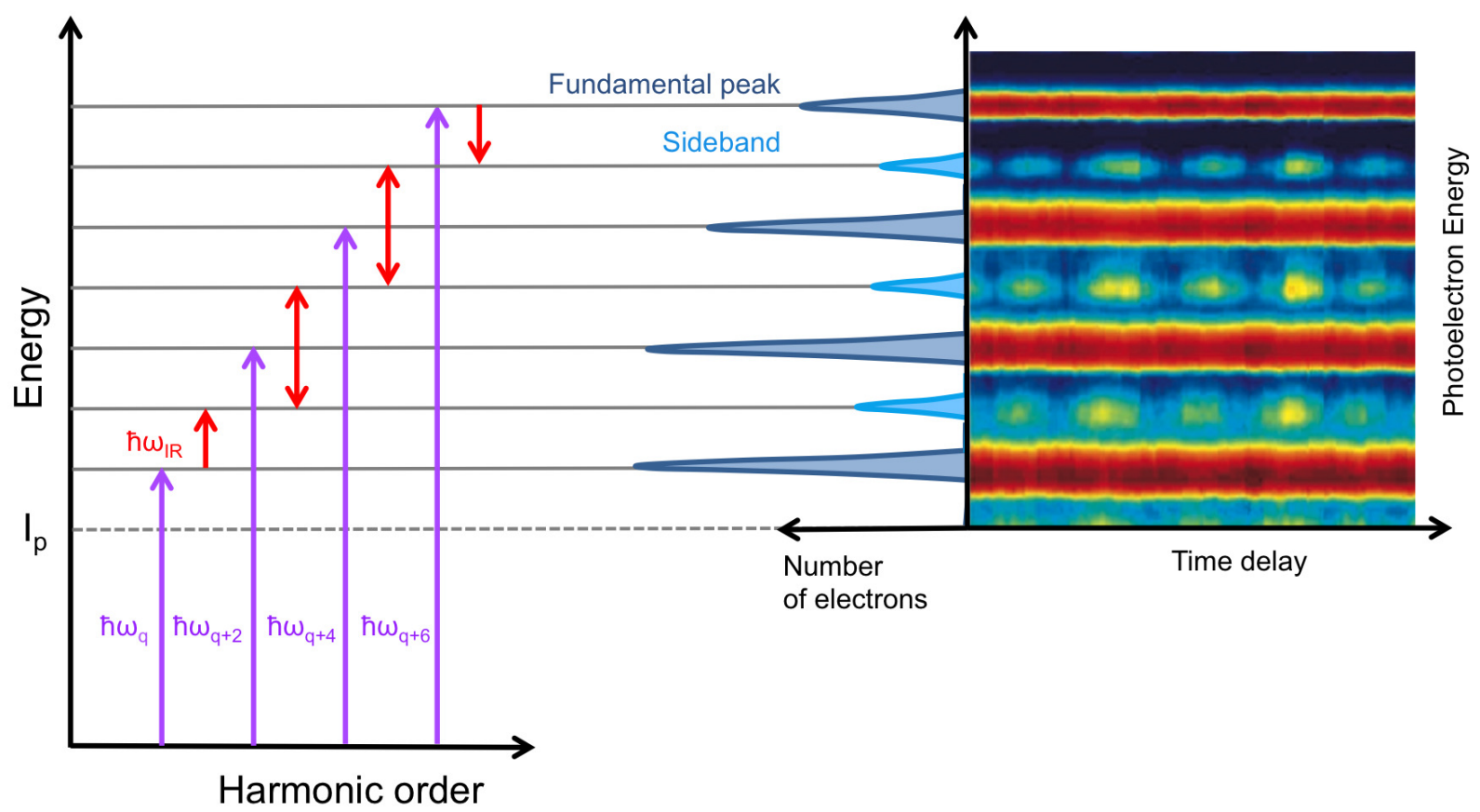

Figure 9: RABBITT scheme. Left panel: odd order harmonics (purple arrows) overcome the ionization potential $\left(\mathrm{I}_{p}\right)$ and create a photo-electron signal (blue peaks). Further absorption/emission of an IR photon (red arrows) creates sideband photo-electrons (light blue peaks). Right panel: upon changing the time delay between EUV and IR pulses, the sideband amplitude oscillates at twice the frequency of the IR field.

of the harmonics as predicted by the semiclassical model (see Sect. 2.2). Equation 24 also allows one to determine the relative phase $\Delta \phi_{\text {atom }}^{f}$ when the relative phase between consecutive harmonics $\Delta \phi_{q}$ is known. Since, as proposed by Wigner, ${ }^{152}$ the phase is directly linked to the time delay a particle experiences when scattered by a potential through the formula $\tau_{\text {atom }}=d \phi_{\text {atom }}^{f} / d \omega$, the RABBITT technique has also been used to measure relative photoelectron emission time delays in atoms, ${ }^{11,12,153-155}$ molecules, ${ }^{156,157}$ and solids. ${ }^{19}$.

\subsubsection{Attosecond streak camera}

In general, a complete characterization of a light pulse can be accomplished in the frequency domain by measuring the spectral intensity $I(\omega)$ and the spectral phase $\phi(\omega)$. While $I(\omega)$ can be easily measured with a spectrometer, the measurement of $\phi(\omega)$ typically requires sophisticated techniques based on non-linear effects. However, due to the relatively low 
energies achieved in the attosecond pulse generation process, nonlinear techniques cannot be immediately extended to the characterization of isolated attosecond pulses. As for trains of attosecond pulses, isolated attosecond pulses can be characterized using a cross-correlation method based on the measurement of the electrons photoionized by the EUV pulse in the presence of an IR field.

The first characterization of isolated attosecond pulses was performed in 2001 by Hentschel et $a l$ with the implementation of the attosecond streak-camera. ${ }^{7}$ In this ex-situ technique, the EUV pulse produces an electron wave-packet by photoionization in the presence of an IR pulse (streaking pulse), weak enough not to ionize atoms, but strong enough to impart substantial momentum to the photoelectrons liberated by the EUV pulse (see Fig. 10(a)-(b)). Under the assumption that the dipole transition matrix element does not vary significantly (in phase and amplitude) over the energy range covered by the attosecond pulse spectrum, the electron wave packet can be considered as a replica of the attosecond pulse. The vector potential $\mathbf{A}$ of the IR field modulates in time the phase of the electron wave packet as: ${ }^{158,159}$

$$
\begin{aligned}
\Phi(t) & =-\int_{t}^{+\infty} d t^{\prime}\left[\mathbf{v} \cdot \mathbf{A}\left(t^{\prime}\right)+\mathbf{A}^{2}\left(t^{\prime}\right) / 2\right] \\
& =-\int_{t}^{+\infty} d t^{\prime} U_{p}\left(t^{\prime}\right)+\frac{\sqrt{8 W U_{p}}}{\omega_{L}} \cos \theta \cos \omega_{L} t-\left(U_{p} / 2 \omega_{L}\right) \sin 2 \omega_{L} t
\end{aligned}
$$

where $\omega_{L}$ is the laser frequency, $U_{p}$ is the ponderomotive potential of the IR pulse, $\theta$ is the angle between the initial electron velocity $\mathbf{v}$ and the vector potential $\mathbf{A}$ and $W=p^{2} / 2$ is the final kinetic energy of the electron. The modulation of the temporal phase corresponds to a shift of the energy components of the electron wave packet (see Fig. 10(c)). Thus, by measuring the streaking effect on the photoelectron distribution for different time delays it is possible to estimate the temporal duration of the EUV pulse. As for the RABBITT technique, the measurement of the phases associated with streaking signals resulting from different ionization channels has been used to determine relative photoelectron emission time delays using the phase-time correspondence discussed in the previous section ${ }^{154}$. 

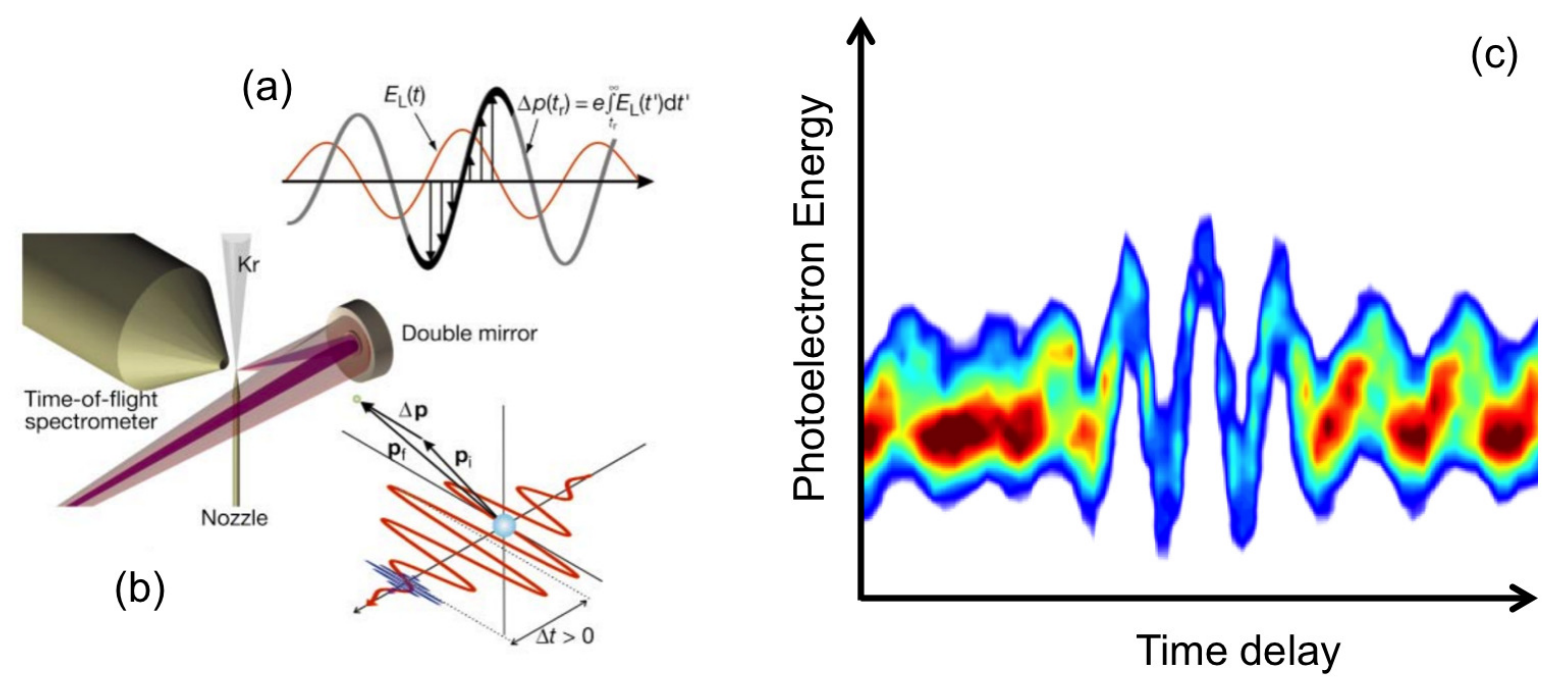

Figure 10: Attosecond streak camera. (a) A photoelectron is released after EUV ionization with initial momentum $\mathbf{p}_{\mathbf{i}}$; an IR probe pulse transfers additional momentum $\Delta \mathbf{p}(t)=e \int_{t}^{\infty} E_{L}\left(t^{\prime}\right) d t^{\prime}$ to the photoelectron, which depends on the phase and amplitude of the IR electric field, resulting in a final momentum $\mathbf{p}_{\mathbf{f}}=\mathbf{p}_{\mathbf{i}}+\Delta \mathbf{p}$. (b) In a typical streaking measurement, the kinetic energy distribution of the ejected photoelectrons is measured with a time-of-flight spectrometer, aligned parallel to the polarization direction of the IR pulse, as a function of the delay between the EUV and the IR pulse. (c) Streaked spectra of photoelectrons: the acquired spectrogram follows the shape of the vector potential of the IR pulse. From ref. 8 with permission. Copyright 2002 Macmillan Publishers Limited.

In 2005 Mairesse and Quére suggested that in the attosecond streak camera the IR pulse is used as a phase gate allowing for frequency resolved optical gating (FROG)-like measurements. ${ }^{160}$ The proposed idea allowed the authors to create for the first time a strong analogy between a very efficient tool of ultrafast optics and attosecond metrology. FROG is indeed a widely used technique for the full characterization of visible pulses. ${ }^{161}$ In this technique, a temporal gate is applied to the pulse to be characterized and the spectrum of the pulse is measured by varying the delay with the gate. This approach provides a two-dimensional spectrogram (FROG trace), given by:

$$
S(\omega, \tau)=\left|\int_{-\infty}^{+\infty} d t G(t) E(t-\tau) e^{i \omega t}\right|^{2}
$$

where $G(t)$ is the temporal gate and $E(t-\tau)$ is the electric field to be characterized. From 
such a spectrogram, a complete temporal characterization of the electric field can be achieved using well established inversion algorithms. The extension of FROG to the attosecond domain has been dubbed as FROG-CRAB (FROG for Complete Reconstruction of Attosecond Bursts). The streaking spectrogram, measured in a given observation direction upon scanning the time delay between the EUV and the IR pulses, is given by:

$$
S(\mathbf{v}, \tau)=\left|\int_{-\infty}^{+\infty} d t e^{i \Phi(t)} \mathbf{d}_{\mathbf{p}-\mathbf{A}(t)} \cdot \mathbf{E}_{E U V}(t-\tau) e^{i\left(W+I_{p}\right) t}\right|^{2}
$$

where $\Phi(t)$ is the phase given in eq $25, \mathbf{d}$ is the dipole matrix element, $I_{p}$ is the ionization potential of the medium and $\mathbf{E}_{E U V}$ is the field to be characterized. As can be seen from the comparison between eq. 26 and eq. 27, the streaking spectrogram corresponds to a FROG trace where the temporal gate is a pure phase gate: $G(t)=e^{i \Phi(t)}$. The same iterative inversion algorithms used for optical FROG, such as the very efficient Principal Component Generalized Projections Algorithm (PCGPA), ${ }^{162}$ have been successfully applied to CRAB traces and isolated attosecond pulses produced with several gating techniques have been fully characterized ${ }^{133,147,163-165}$ (see Fig. 11).

Finally, another ex-situ technique, which differs from the attosecond streak camera only for the intensity of the IR field, is the Phase Retrieval by Omega Oscillation Filtering (PROOF). ${ }^{167}$ As for the RABBITT technique, this method is based on the presence of a weak perturbing IR field, whose action on the photoelectrons produced by EUV photoionization can be fully treated using the lowest-order perturbation theory. Although the physical interpretation of PROOF is very close to RABBITT, there is a fundamental difference between the two techniques: in the case of PROOF, the EUV spectrum is no longer composed by discrete peaks but by a continuum distribution. It is possible to demonstrate that, in this case, the photoelectron spectrum corresponds to the superposition of three oscillating terms:

$$
I\left(\omega_{\nu}, \tau\right)=I_{0}(\nu)+I_{\omega_{L}}(\nu, \tau)+I_{2 \omega_{L}}(\nu, \tau)
$$



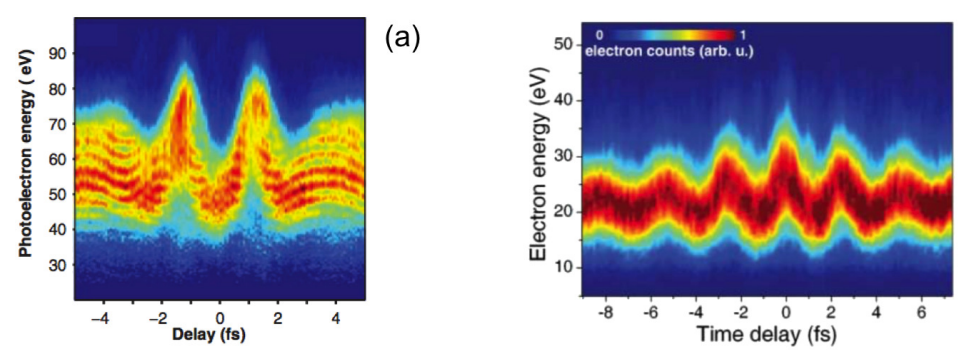

(b)

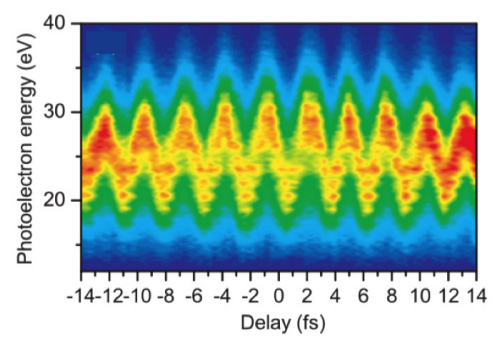

(c)
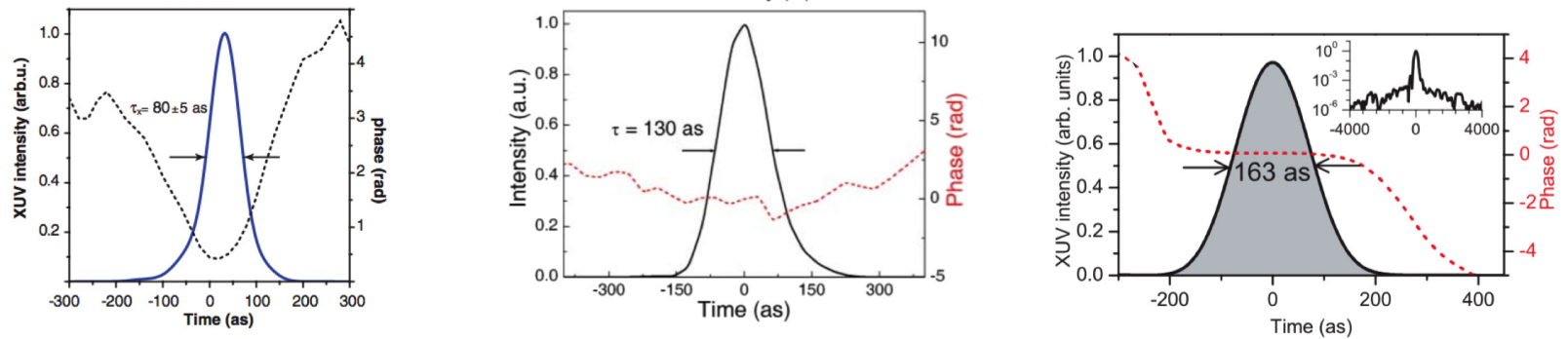

Figure 11: Attosecond streaking spectrograms (top) and reconstructed intensity profile and phase of the isolated attosecond pulses (bottom) generated with spectral filtering (a), polarization gating (b) and GDOG (c). From refs. [ 133,164,166] with permission. Copyright 2006, 2008 The American Association for the Advancement of Science (AAAS). Copyright 2010 American Physical Society.

where $\omega_{\nu}$ is a frequency of the EUV spectrum and $\omega_{L}$ is the frequency of the IR pulse. These oscillating terms derive from the quantum interference of the continuum states caused by the presence of the IR dressing field. The amplitude and phase of these oscillations strongly depend on the amplitude and phase of each interfering spectral component. After isolating from the spectrum the component oscillating at the laser frequency $\omega_{L}$, the interference is uniquely related to the following contributions: direct photo-absorption of an EUV photon with frequency $\omega_{\nu}$, direct photo-absorption of an EUV photon with frequency $\left(\omega_{\nu}+\omega_{L}\right)$ with emission of an IR photon, and direct photo-absorption of an EUV photon with frequency $\left(\omega_{\nu}-\right.$ $\left.\omega_{L}\right)$ with absorption of an IR photon. Thus the interference signal encodes the spectral phases $\phi\left(\omega_{\nu}\right), \phi\left(\omega_{\nu}+\omega_{L}\right)$ and $\phi\left(\omega_{\nu}-\omega_{L}\right)$, which can be uniquely retrieved by matching the measured sinusoidal oscillations as a function of the time delay $\tau$ for each spectral component $\omega_{\nu}$. Since the reconstruction procedure does not rely on the central momentum approximation as in the case of FROG-CRAB, this method is particularly suitable for characterizing attosecond pulses with ultrabroad bandwidths. By using this technique, in 2012 the measurement of 
67-as pulses produced by the DOG technique has been demonstrated. ${ }^{124,168}$

\subsubsection{Optical methods}

Alternatively to the ex-situ measurements described above, all-optical methods based on the measurement of the EUV photon spectrum for the reconstruction of the phase of the attosecond pulse have been proposed. As briefly mentioned at the beginning of this section, these are in-situ measurements, meaning that the characterization of the attosecond pulse is performed in the same medium where it is generated. ${ }^{169}$ The first in-situ approach was proposed by Dudovich and co-authors in $2006:{ }^{16}$ in this experiment the HHG process was gently perturbed by the presence of a weak and collinear second-harmonic beam. In the HHG process the produced EUV spectrum is the result of the interference between two consecutive half-cycles of the fundamental field, which, due to the symmetry of the field, leads to the generation of odd-order harmonics. The presence of a weak second harmonic field breaks the symmetry, thus resulting in the generation of even harmonics of the fundamental. In this case, the phase accumulated by the electron is enhanced in the half cycle where the fundamental and the second-harmonic fields are in phase, and suppressed in the adjacent half cycles where the two fields are in opposite phase. By changing the delay between the fundamental and its second harmonic, their relative phase is varied inducing a modulation in the intensity of the even harmonics signal (see Fig. 12). While the periodicity of the modulation is the same for all even harmonics, the phase of the modulation is different for each even harmonic. Indeed the phase of the electron wave packet accumulated along the electron trajectory is affected by the weak second-harmonic field, and it changes from one harmonic to another. From the phase-measurement of the modulation of the even harmonics, the emission times can be reconstructed and consequently the phase of the unperturbed electron wave packet can be measured. In analogy with the previously described techniques, in this case the temporal phase gate is directly applied on the re-collision process by the weak second-harmonic field with attosecond resolution. 


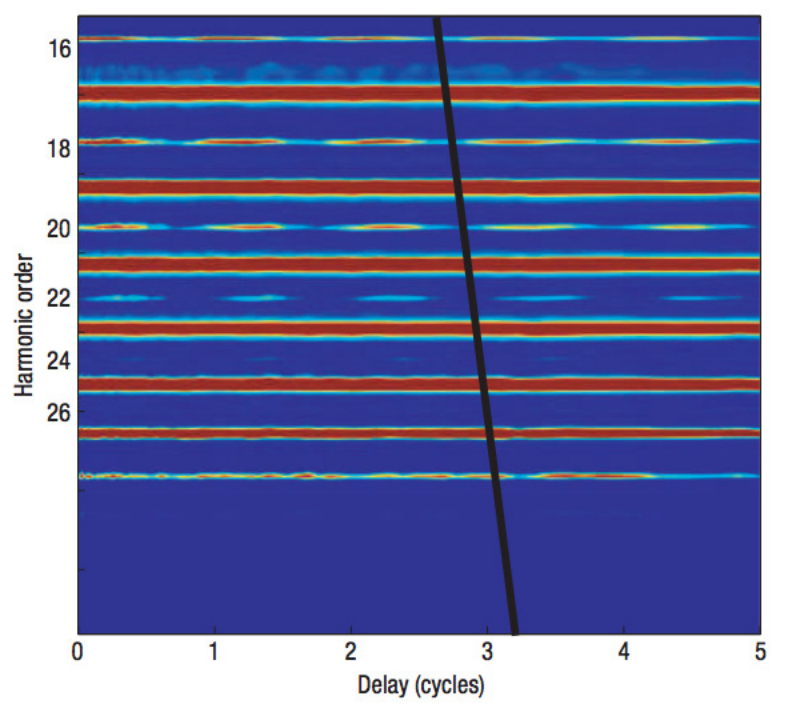

Figure 12: Harmonic spectrum acquired as a function of the relative delay between the fundamental and the second harmonic field. The even harmonics show a periodic modulation with a phase that depends on the harmonic order. From ref. [16] with permission. Copyright 2006 Macmillan Publishers Limited.

Recently, a similar approach has been implemented to characterize isolated attosecond pulses. ${ }^{170}$ The general idea is the same as described above: a weak field (signal field) is used to gently perturb the electron trajectories. This perturbation changes the phase of the EUV radiation and the induced phase shift $\sigma_{\varepsilon}$ is proportional to the signal field. If the signal field is superimposed on the fundamental field with a small angle $\theta_{p}$, the perturbation can change the wavefront of the EUV radiation, leading to deflection of the EUV beam. This macroscopic response can be used to make $\sigma_{\varepsilon}$ observable, as the change of the propagation angle can be measured from the angularly resolved EUV spectrum (see Fig. 13). The vertical displacement of the the EUV spectrum measured in the far-field as a function of the delay between the fundamental pulse and the signal pulse provides the following spectrogram:

$$
S(\theta, \tau)=\left|\int_{-\infty}^{\infty} d y E_{\mathcal{E}}^{n e a r}(y) G_{\mathcal{E}}\left(y-c \tau / \theta_{p}\right) \exp \left(i k_{\mathcal{E}} \theta y\right)\right|^{2}
$$

where $\theta$ is the propagation angle of the EUV radiation, $\tau$ is the time delay, $k_{\varepsilon}$ is the EUV wavenumber and $E_{\mathcal{E}}(y)$ is the complex amplitude of the unperturbed EUV radiation gen- 
erated by the short and long quantum paths as a function of the vertical coordinate $y$. In this case the function $G_{\mathcal{E}}\left(y-c \tau / \theta_{p}\right)$, which describes the amplitude and phase modulation induced by the perturbing field, corresponds to a spatial gate. Since eq 29 describes a general FROG spectrogram, an iterative inversion algorithm can be used to retrieve the EUV near field $E_{\mathcal{E}}^{\text {near }}(y)$. This method has been implemented not only to completely characterize the EUV pulses (with both short and long quantum path contributing to the generation process), but also to retrieve the electric field of the perturbing pulse, as demonstrated by Kim et al. ${ }^{171}$ In their experiment, 5-fs 750 -nm laser pulses were split into two beams: one beam was used to generate isolated attosecond pulses with the polarization gating technique, while the other beam was used as a weak signal field $E_{s}(t)$ to be characterized. The two beams were then combined at the focus with a small angle and the EUV spectrum was measured with an EUV spectrometer in the far-field. The energy range between $36.9 \mathrm{eV}$ and $40.2 \mathrm{eV}$ was integrated for each time delay, providing a spectrogram with a center of gravity corresponding to $d E_{s}(t) / d t$. This technique, called petahertz optical oscilloscope, represents a powerful alternative to attosecond streaking to characterize petahertz fields.

\subsection{Attosecond spectroscopy techniques}

The generation and complete characterization of attosecond pulses have opened the way to real-time observation and steering of electronic dynamics on the atomic scale. The most conventional approach to perform a time resolved measurement is to use a first pulse (pump) to excite the system and a second delayed pulse (probe) to detect the pump-induced dynamics. Due to the relatively low conversion efficiency of the attosecond pulse generation process, for several years the typical pump-probe scheme in attosecond science has made use of an EUV attosecond pulse in combination with an IR femtosecond pulse. However, this is no longer the only adopted scheme, as the recent development of high-photon flux EUV sources has allowed attosecond-pump attosecond-probe approaches to be implemented. Moreover, in-situ techniques based on the HHG process itself have been used to obtain spectroscopic 

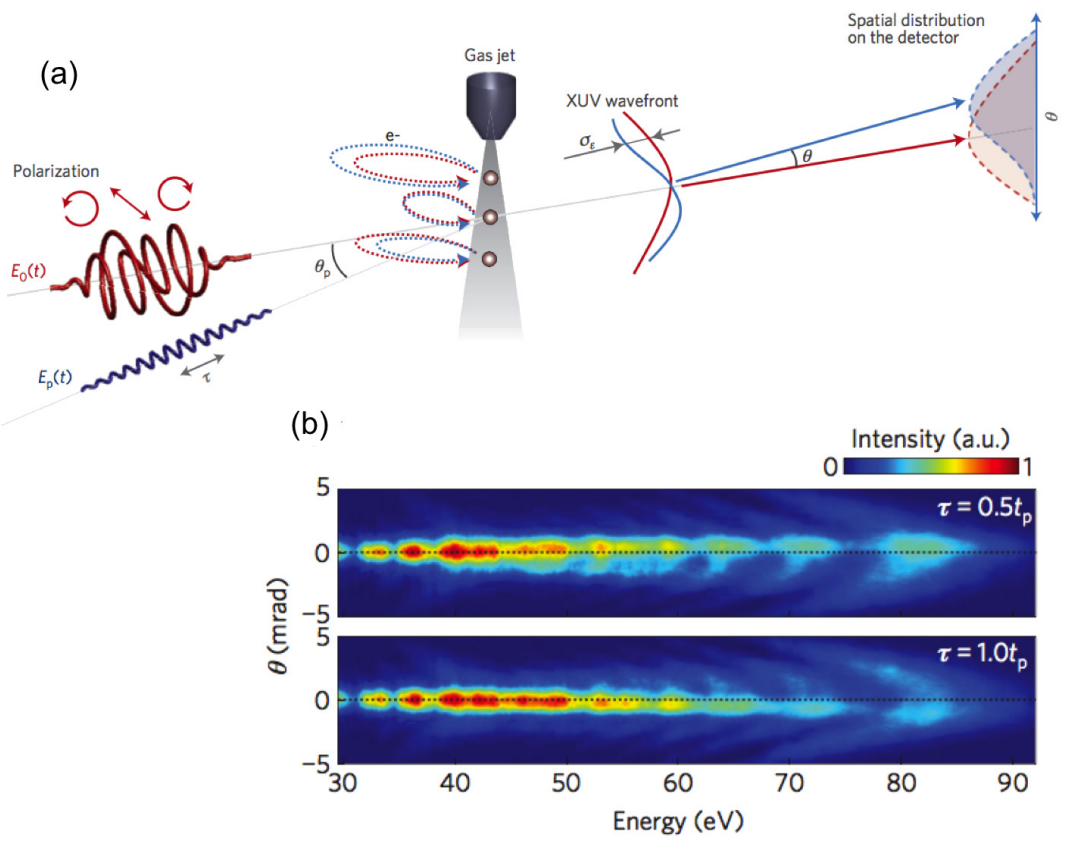

Figure 13: (a) Illustration of the in-situ technique for isolated attosecond-pulse characterization. The second harmonic field modulates the electron trajectories and, as a result, the generated EUV radiation exhibits a modified wavefront resulting in a change in the propagation angle. (b) Spatially resolved EUV spectra measured in the far-field for different time delays: the spectrum is shifted up (top panel) or down (bottom panel) depending on the delay. From ref. [ 170] with permission. Copyright 2013 Macmillan Publishers Limited.

information on the generating target with attosecond time resolution. Due to the high photon energy of the attosecond pulses (ranging from the EUV to the soft-X spectral region), the excitation of the sample almost inevitably leads to inner valence or core photoelectron emission, followed by a rearrangement of the electronic system. The electron reorganization can give rise to Auger decay, shake-up processes, charge transfer or charge migration in large molecules. In this section we will summarize various experimental schemes proposed and demonstrated for attosecond time resolved spectroscopy. As mentioned above, both streaking and RABBITT techniques have also been used for spectroscopic applications, for example for the measurement of delays in photoemission in atoms, molecules and solids. 


\subsubsection{Attosecond electron/ion spectroscopy}

The release of one or more electrons upon the interaction of attosecond pulses with atoms and molecules, in general, leaves the ionized system in a non-stationary state that will evolve in time initiating a dynamics that can take different forms depending on the target. A large variety of methods exist to obtain spectroscopic information about electrons and ions produced by the EUV induced photoionization of atoms and molecules. The simplest way to collect charged particles and obtain their kinetic energy is to measure the time required by each particle to fly along a drift-tube. For this reason, such a detection technique is called Time of Flight (TOF) spectroscopy. TOF spectrometers are typically equipped with high count-rate particle detectors and they can be designed for a large variety of purposes including measuring the kinetic energy distribution of photo-electrons (electron TOF spectrometer) ${ }^{172}$ or the mass of the produced photo-ions (ion mass TOF spectrometer). ${ }^{173}$ To improve the collection efficiency of a conventional electron TOF spectrometer, magnetic bottle spectrometers have been designed. ${ }^{174}$ The essence of a magnetic bottle electron spectrometer is a strong inhomogeneous magnetic field around the source point, which is designed to efficiently redirect the photoelectrons into a weak homogeneous magnetic field that guides them into the drift tube towards the detector. Magnetic bottle spectrometers allow the detection of single-shot electron spectra. More sophisticated multi-particle electron-ion imaging spectrometers have been also used for attosecond experiments including Velocity Map Imaging (VMI) spectrometers and Reaction Microscopes (REMI). VMI spectrometers allow for the measurements of both the kinetic energy and the angular distributions of electrons or ions with high count rates. ${ }^{175}$ In a VMI spectrometer, a static electric field is generated by the combination of two electrodes (repeller and extractor), and the charged particles are projected onto a position sensitive microchannel plate $(\mathrm{MCP})$ detector combined with a phosphor screen and a fast $\mathrm{CCD}$ camera for the acquisition of a $2 \mathrm{D}$ image. The $2 \mathrm{D}$ image directly reflects the $3 \mathrm{D}$ momentum distribution projected onto the detector plane, and the 3D velocity distribution of the particles can be retrieved from the 2D-projected images by means of an inverse-Abel 


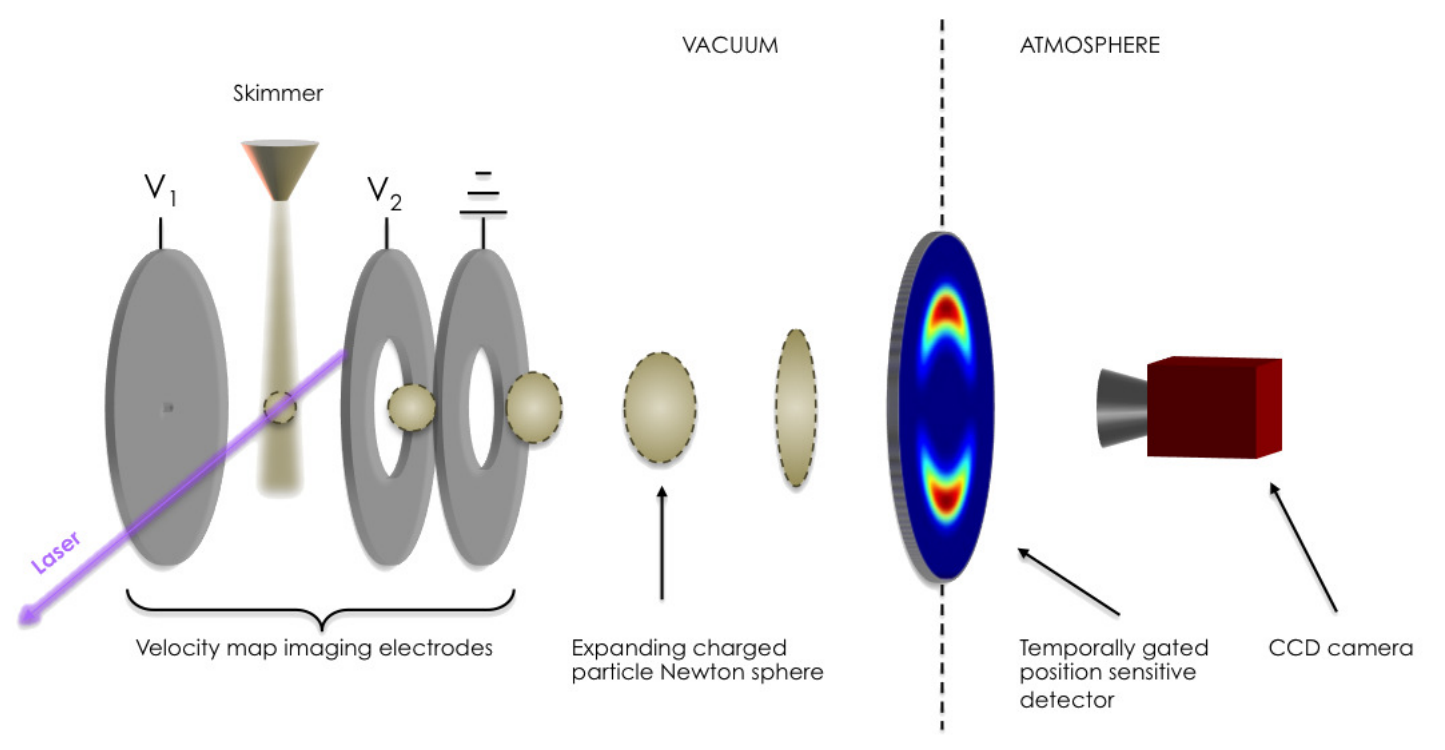

Figure 14: Schematic of a VMI arrangement used to perform attosecond electron/ion spectroscopy. $\mathrm{V}_{1}$ and $\mathrm{V}_{2}$ correspond to the voltages applied to the repeller and extractor electrodes, respectively. The 3D momentum distribution is projected on the detector (MCP) plane and the $2 \mathrm{D}$ image is acquired with a CCD camera.

transform. ${ }^{176}$ VMI spectrometers are typically used for studying photoionization dynamics of atoms and molecules in the laboratory frame. As will be shown in the following sections, VMI detection has been implemented in combination with attosecond sources for example to measure electron localization in $\mathrm{D}_{2}$ molecules induced by few-cycle CEP stabilized laser pulses, ${ }^{33}$ to investigate the dissociative ionization dynamics of $\mathrm{N}_{2}{ }^{177}$ and to study timedependent dipole effects in $\mathrm{CO}_{2}$ and $\mathrm{C}_{2} \mathrm{H}_{4} \cdot{ }^{178}$ Since attosecond sources typically provide a reduced photon flux, a lot of effort has been put for increasing the density of the gas target in the interaction region and tremendous progress has been achieved by integrating the gas injection system in the repeller electrode of the spectrometer. ${ }^{179}$ Although the combination of a VMI spectrometer with techniques for impulsive alignment and orientation of molecules allows for measurements of photoelectron distributions also in the molecular frame, a more detailed information on the kinematics of the experiment can only be achieved by detecting photo-fragments in coincidence. 
The coincidence detection of multiple ions and electrons becomes possible with REMI, ${ }^{180}$ which measures the 3D momentum distribution of all charged fragments produced from the target. This spectrometer is based on the combination of cold-target recoil ion momentum spectroscopy (COLTRIMS) with electron imaging. ${ }^{181,182}$ The general design of a REMI requires a well collimated beam of cold atoms or molecules, usually provided by a supersonic gas expansion, which is crossed by the laser beam. A weak electrostatic field is applied for extraction of the produced charged target fragments, the electrodes are designed to accelerate electrons on one side and ions on the other side of the spectrometer (see Fig. 15). On both sides of the target area, a field-free drift path and a large area position sensitive detector are located to measure the momentum distribution of both electrons and recoil ions in coincidence. In order to achieve a better spatial confinement of electrons, a weak homogenous magnetic field (generated by a pair of large Helmholtz coils) is superimposed along the spectrometer axis. COLTRIMS has been successfully combined with an attosecond beamline (attoCOLTRIMS) and streaking measurements performed in coincidence have allowed the delays in photoemission from Ar and Ne atoms to be retrieved. ${ }^{183}$

\subsubsection{High-order harmonic spectroscopy}

When an electron is ionized from an atom or a molecule by an intense laser pulse, it is inevitably steered by the optical field in the continuum before recolliding back with the parent ion. The recollision step may lead to recombination and emission of a photon (HHG) or to laser-induced electron diffraction (LIED). ${ }^{184,185}$ Since the wavelength of a typical recolliding electron matches the size of the valence electron orbitals and the recollision occurs within a sub-cycle of the driving laser field, both processes provide extreme spatial (Ångström) and temporal (attosecond) resolution. Thus, the detection of either the emitted photon or the electron diffracted by the ion can be used as a time and spatially resolved spectroscopic technique in which the time between ionization and recollision can be viewed as the pumpprobe delay. In the following, we will focus on the spectroscopic information that can be 


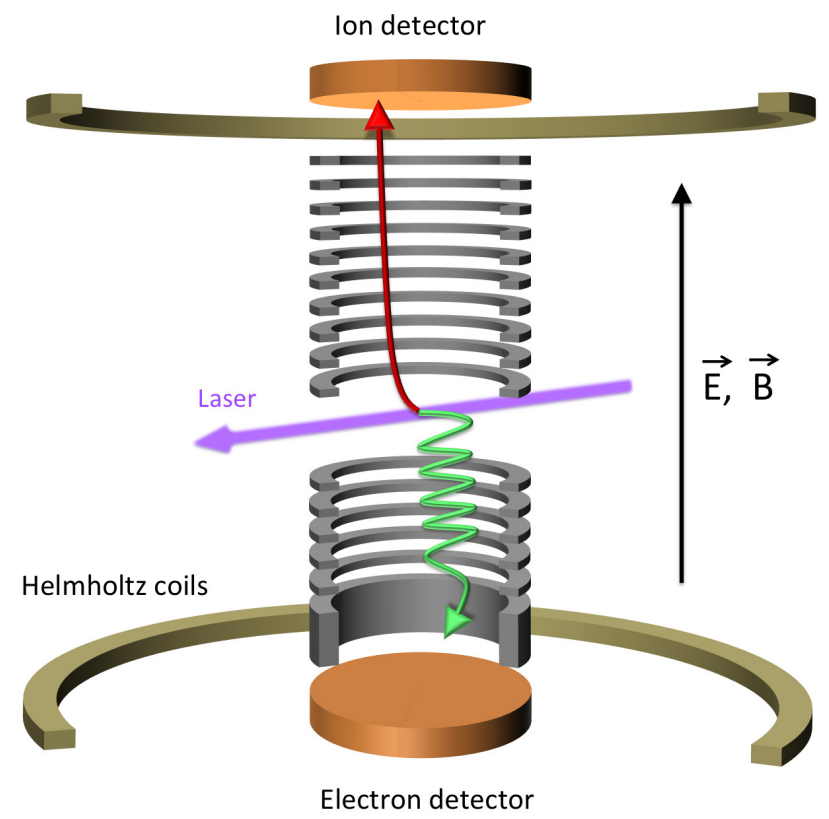

Figure 15: Schematic of a reaction microscope used for coincidence measurements. Ions (red arrow) and electrons (green arrow) produced by laser-induced ionization of a rotationally cold supersonic gas jet are extracted via electric and magnetic fields respectively and detected in coincidence with position-sensitive detectors in conjunction with delay-line anodes.

extracted from the EUV spectrum emitted during the HHG process, while a more detailed description of the laser induced electron diffraction (LIED) technique will be given in Sec. 7.2 .

According to the three-step model, the EUV spectrum emitted via the HHG process contains information on both the ionization and the recollision processes as well as on the molecular structure and on the temporal evolution of the parent cation (vibrational and rotational dynamics and hole migration) between ionization and recombination events. ${ }^{98,185-188}$ As already mentioned in Sec. 2.1, in the HHG process a given photon energy corresponds to two possible electron trajectories (long and short). However, a proper choice of the phase matching conditions allows the selection of a single set of trajectories (typically the short ones), thus creating a one-to-one correspondence between a specific photon energy and the electron traveling time. Thus the evolution of the ionized system between electron ionization and recollision can be probed by the acquisition of a single EUV spectrum, since it contains a broad range of energies corresponding to different time intervals spent by the electron in 
the continuum. ${ }^{189,190}$ Following this idea, in 2006 Baker et al demonstrated that ultrafast molecular dynamics can be monitored with attosecond time resolution by chirp-encoded recollision (PACER) technique and both nuclear dynamics in $\mathrm{H}_{2}$ and structural rearrangement in $\mathrm{CH}_{4}$ and $\mathrm{CD}_{4}$ could be measured. ${ }^{191}$ Nevertheless, several theoretical works going beyond the Born-Oppenheimer approximation have shown that the coupling between electronic and nuclear motions in molecules containing light nuclei can make the analysis more complicated than in atoms and can even lead to the appearance of even harmonics in the spectrum. ${ }^{192-194}$

One of the fundamental challenges in applying high-order harmonic spectroscopy (HHS) to resolve electron and nuclear rearrangement is the need to decouple temporal and spatial information encoded in the HHG spectrum, including electron and nuclear dynamics. This requires characterization of all the steps of the laser-molecule interaction: ionization, electron-hole dynamics, and radiative electron-hole recombination. To this end, an advanced scheme for multi-dimensional HHS has been proposed and developed, based on the use of phase-locked multi-colour laser fields. ${ }^{195,196}$ In such scheme, the electron trajectories are properly manipulated by controlling the relative phases and polarizations between the two different fields. This idea was implemented by Shafir and co-authors by combining an intense 800-nm laser pulse with a weak and orthogonally polarized 400-nm pulse. ${ }^{197}$ The two laser pulses were synchronized and the relative phase between them was finely controlled. As a result of the orthogonal polarization of the two fields, the electron liberated in the continuum is laterally displaced by the weak second harmonic component. As a direct consequence of this lateral shift, the electron recollision is suppressed for a large number of trajectories and photon emission occurs only for a specific photon energy (gating in the ionization step). By adjusting the two-color delay the temporal gate to the HHG process is shifted at different energies and a unique relationship between ionization times and emitted photon energies can be retrieved. An independent relationship between recombination times and photon energies is also obtained by measuring the ratio between neighboring even and odd harmonics as a function of the phase offset. As can be observed in Fig. 16, by combining these two 
measurements, the authors have been able to extract experimentally ionization and recombination times. The reconstructed recombination times agree well with the prediction of the semiclassical model, while the reconstructed ionization times deviate from the semiclassical prediction and they can only be reproduced with a quantum model. A quantum description of the ionization is indeed required to take into account tunneling and the non-zero velocity of the electron as it exits the barrier. The same measurement performed in $\mathrm{CO}_{2}$ allowed the contribution from two different molecular orbitals to be identified and a precise estimation of the relative ionization delays to be obtained. The results obtained with multi-dimensional HHS are of particular relevance, since they show the possibility to gain access to the individual steps of the generation process, opening new perspectives in the investigation of multi orbital contributions in the strong field ionization process.

\subsubsection{Attosecond non-linear spectroscopy}

Due to the low conversion efficiency of the attosecond pulse generation process, until recently most EUV sources were lacking sufficient pulse energy to perform EUV-pump EUV-probe non-linear measurements. Although, as mentioned above, most of the experimental efforts in attosecond science have been restricted to EUV-IR pump-probe schemes, the systematic development of HHG sources providing high photon flux ${ }^{141,198-202}$ have paved the way to the extension of attosecond spectroscopy to the nonlinear regime.

The first experimental demonstration of a nonlinear multiphoton process, exploiting the use of a single harmonic of the EUV spectrum, was provided in 2005 by Nabekawa et al. ${ }^{203}$ In this experiment the combination of loosely focusing geometry and a long interaction length allowed scaling the HHG process to the $\mu \mathrm{J}$ energy range. The detection of doubly charged $\mathrm{He}^{2+}$ indicated the first observation of a nonlinear optical process (two-photon absorption) occurring in the medium. The nonlinear process also allowed performing the first interferometric autocorrelation. To this end, a delayed pair of harmonic pulses was produced by spatially dividing the harmonic beam with two beam separators of $\mathrm{SiC}$ substrates. One of 

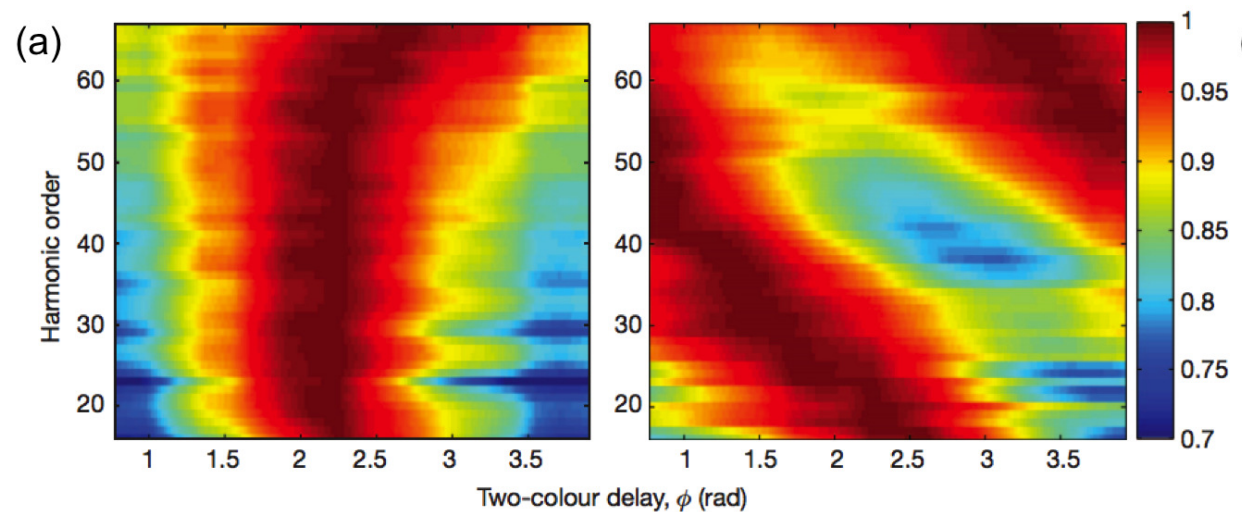

(b)

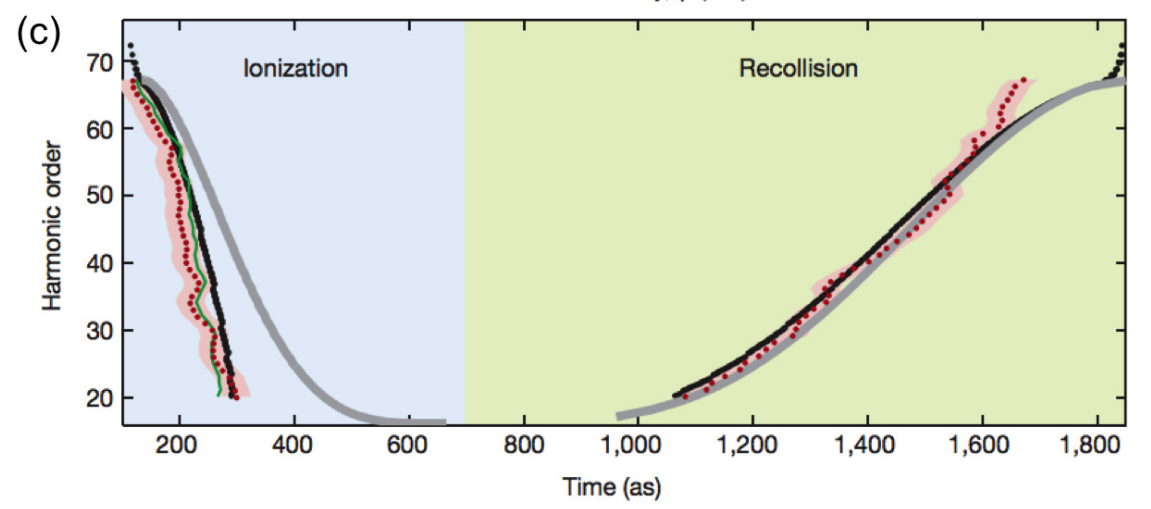

Figure 16: (a) Harmonic signal acquired as a function of the two-color delay. (b) Ratio between adjacent even and odd harmonics as a function of the two-color delay. (c) Ionization and recombination times (red dots) reconstructed from (a) and (b), respectively. Theoretically predicted ionization and recombination times are shown in grey (semiclassical model) and black (quantum stationary solution). From ref. [ 197] with permission. Copyright 2012 Macmillan Publishers Limited.

the divided pulses was then delayed or advanced with respect to the other pulse by moving one of the separators placed on a translation stage with a piezo-actuator. Exploiting this autocorrelator setup and the two-photon double ionization of He, a pulse duration of 8 fs was retrieved. Following this pioneering work, a similar experimental setup has been used to characterize attosecond pulse trains exploiting different physical processes such as two-photon above-threshold ionization in Argon ${ }^{204}$ and Coulomb explosion in $\mathrm{N}_{2}$ induced by two-photon absorption ${ }^{205}$ (see Fig. 17).

As discussed above, the use of high-power many cycle pulses combined with a loosely focusing geometry has rapidly led to the development of intense attosecond pulse trains and to the investigation of nonlinear phenomena in the extreme-ultraviolet spectral region. 


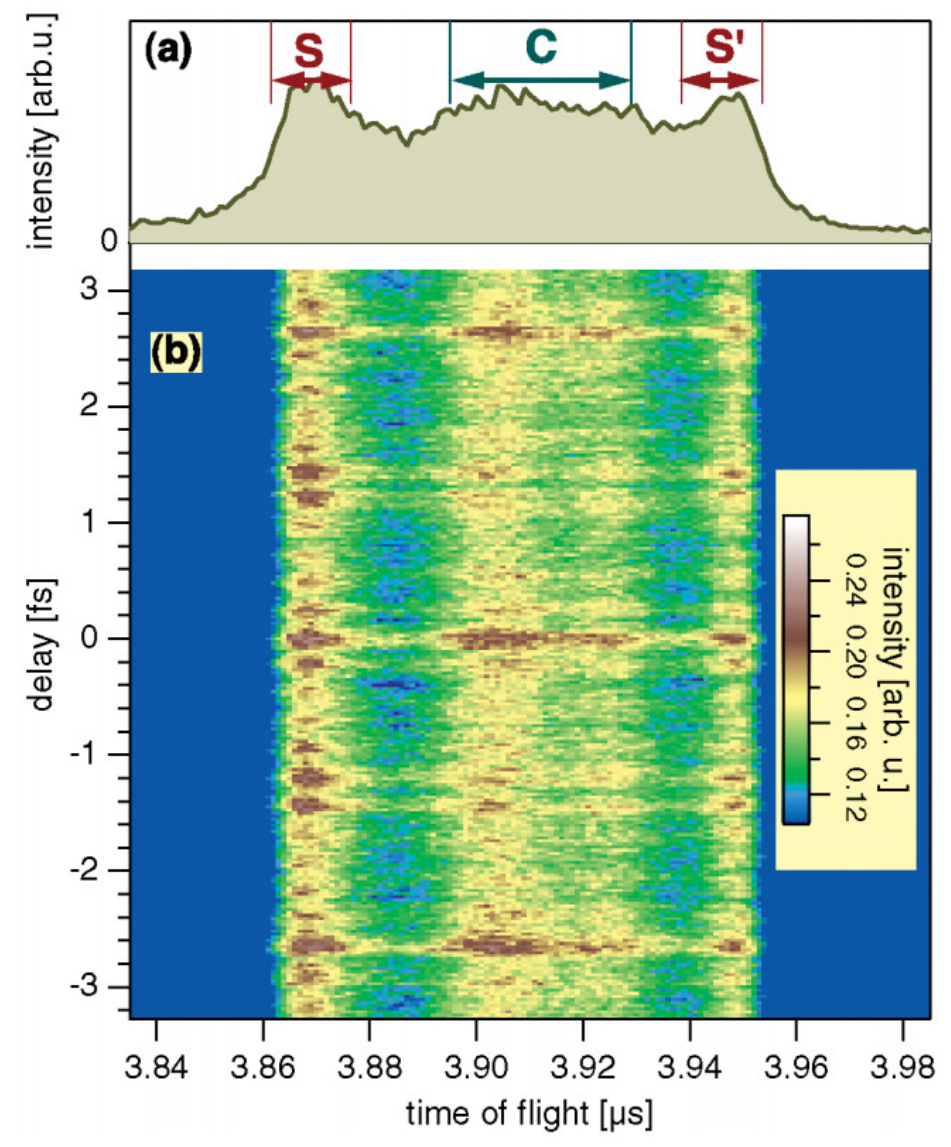

Figure 17: (a) Typical $\mathrm{N}^{+}$time of flight spectrum obtained from the dissociative ionization of $\mathrm{N}_{2}$ induced by two-photon absorption. (b) Time of flight signal measured as a function of the delay between the two EUV attosecond pulse trains. Fast oscillations reveal the interferometric autocorrelation of the attosecond pulse train and a pulse duration of 320 as has been retrieved for each pulse of the train. From ref. [ 205] with permission. Copyright 2006 American Physical Society.

However, most of the initial experiments were conducted with attosecond pulse trains due to the lack of few-optical-cycle laser sources to drive the single attosecond pulse generation process with sufficient efficiency. In 2007 Tzallas and co-workers proposed a temporal gating scheme for 50-fs long driving pulses based on the interferometric modulation of the ellipticity of the driving pulse. The method was dubbed Interferometric Polarization Gating (IPG) and broadband extreme-ultraviolet continuum generation was demonstrated using high peak-power many-cycle pulses. ${ }^{206}$ This approach has enabled the investigation of nonlinear processes in the EUV spectral region on the 1-fs temporal scale. In a first experiment, 
EUV-broadband coherent continuum radiation was produced in Xe gas by IPG. ${ }^{207-209}$ Sec-

ond order autocorrelation was performed by recording the dependence of the $\mathrm{Xe}^{2+}$ signal on the delay between the two EUV pulses and a pulse duration of approximately 1.5 fs was retrieved. Using the sequence of two EUV pulses also allowed for the control and probe of a fast-evolving coherence in the structured continuum of the xenon atom. Furthermore, the measurement revealed that, on the sub-fs time scale, two-photon direct double ionization dominates over the two-photon sequential double ionization process. Following these encouraging results, the investigation of the EUV-induced coupled electron and nuclear dynamics in $\mathrm{H}_{2}$ was subsequently undertaken by exploiting the same experimental apparatus. ${ }^{210}$ Since this is the first ever experiment in which EUV-induced non linear processes have been investigated in a molecule with attosecond resolution, we will come back to it in more detail in section 5.1, where we review the existing literature on small molecular systems.

\subsection{Attosecond experimental techniques without attosecond pulses}

In this section, a few experimental techniques that allow one to achieve attosecond temporal resolution without directly employing attosecond pulses will be discussed. The following methods will be briefly presented: core-hole spectroscopy and the attoclock technique.

\subsubsection{Core-hole spectroscopy}

In contrast to pump-probe measurements, the core-hole clock spectroscopy can achieve extreme temporal resolution (from a few femtoseconds down to a few attoseconds) by performing measurements in the energy domain. The decay time of a core-hole, created by removing an electron from a core state, acts as an ultrafast internal clock, against which an electron transfer process can be measured. ${ }^{211}$ One can distinguish between the normal Auger process, where the system is core ionized and the subsequent decay generates a doubly ionized system ( $2 h$ configuration), and the resonant Auger one, where a core electron is excited to an empty level that further decays to a singly ionized system. The time-scale of core-shell vacancies 


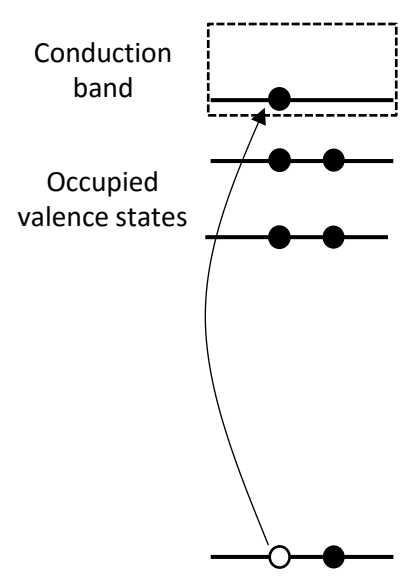

(a) Core-hole excitation

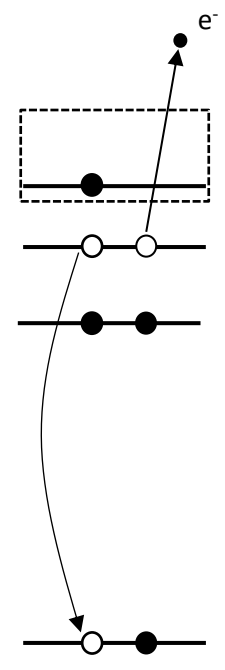

(b) $2 \mathrm{~h} 1 \mathrm{e}$ final state
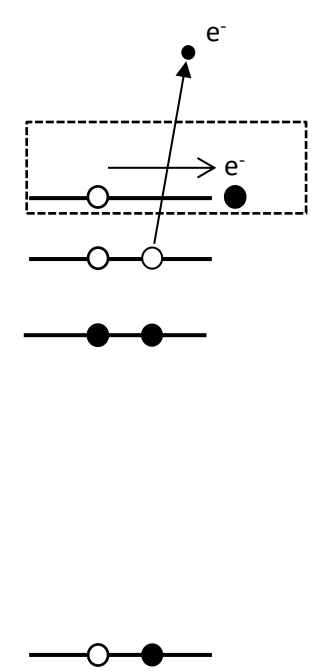

(c) $2 \mathrm{~h}$ final state

Figure 18: (a) A core electron is excited by a soft X-ray photon. (b) Spectator Auger final state (2h1e): the resonantly excited electron remains localized. (c) Normal Auger final state (2h): the excited electron delocalizes.

ranges from $\tau=4$ fs to $\tau=6$ fs (e.g., for oxygen KLL $\tau=4$ fs, ${ }^{212}$ for nitrogen KLL $\tau=5$ fs, ${ }^{212}$ for argon $\mathrm{L}_{3} \mathrm{M}_{4 / 5} \mathrm{M}_{4 / 5} \tau=6 \mathrm{fs}^{213,214}$ ). Much shorter temporal scales can be achieved by employing the Coster-Kronig decay process of core-excited states, with attosecond core-hole lifetimes.

The basic idea at the heart of this experimental technique can be explained as follows (see Ref. [ 211] and references therein). The absorption of a soft X-ray photon excites a core electron, leading to the generation of a state with a core-hole and an electron in a formerly empty valence state (see Fig. 18(a)). The excited core-hole state will decay exponentially via X-ray fluorescence or via Auger processes (with high probability) with a time constant given by the core-hole lifetime, $\tau$. The decay can involve the excited electron, leaving a single hole in the valence shell (participator decay, $1 h$ configuration), which is a minority channel, or two valence electrons (spectator decay), leaving two holes in the valence shell plus the excited electron, a so called 2h1e configuration, which is the most probable process (Fig. 19(b)). There is also the possibility that, as illustrated in Fig. 18(c), prior to core-hole decay, the excited electron is subject to a transfer process (i.e., it delocalizes over many atomic centers 
so that the excited atomic resonance is coupled to a continuum). In this case, the decay process becomes a normal Auger process. In principle, it is possible to understand if the excited electron is in an atomic localized resonance or if it is already delocalized within the core-hole decay time since the normal Auger decay and the spectator Auger decay involving the same final hole configuration are characterized by outgoing Auger electrons with different kinetic energies. ${ }^{215-217}$ In particular, the electron originating from the spectator decay has higher kinetic energy compared to the normal Auger electron as a result of the screening of the Coulomb interaction between the two holes in the final configuration generated by the spectator electron. Indeed, the screening effect reduces the binding energy of the final state, thus leading to a larger kinetic energy of the resulting Auger electron. It is evident that the transfer process of the resonance electron, characterized by an electron transfer time $\tau_{T}$, competes with the core-hole decay process, characterized by the corresponding lifetime $\tau$. It is possible to show that, in the case of exponential decays, the ratio between the intensities of the spectator, $I_{2 h 1 e}$, and normal Auger component $I_{2 h}$ can be written as ${ }^{211,218,219}$

$$
\frac{I_{2 h 1 e}}{I_{2 h}}=\frac{\tau_{T}}{\tau}
$$

Therefore, from the measured intensity ratio $I_{2 h 1 e} / I_{2 h}$ it is possible to obtain the transfer time $\tau_{T}$ in units of $\tau$, which acts as an internal clock. This is particularly important since, as a very good approximation, the core-hole lifetime depends only weakly on the surrounding of a specific atom, ${ }^{212}$ so that its value can be taken from atomic data: in this way it is possible to determine the electron transfer time. ${ }^{218-220}$ Using this procedure and assuming that a statistically significant analysis is possible only if the intensities of the spectroscopic channels are less than one order of magnitude apart, ${ }^{221}$ a reliable determination of the charge delocalization time is possible within the temporal range $0.1 \leq \tau_{T} / \tau \leq 10$. This can be easily understood by plotting, as shown in Fig. 19, the ratio 


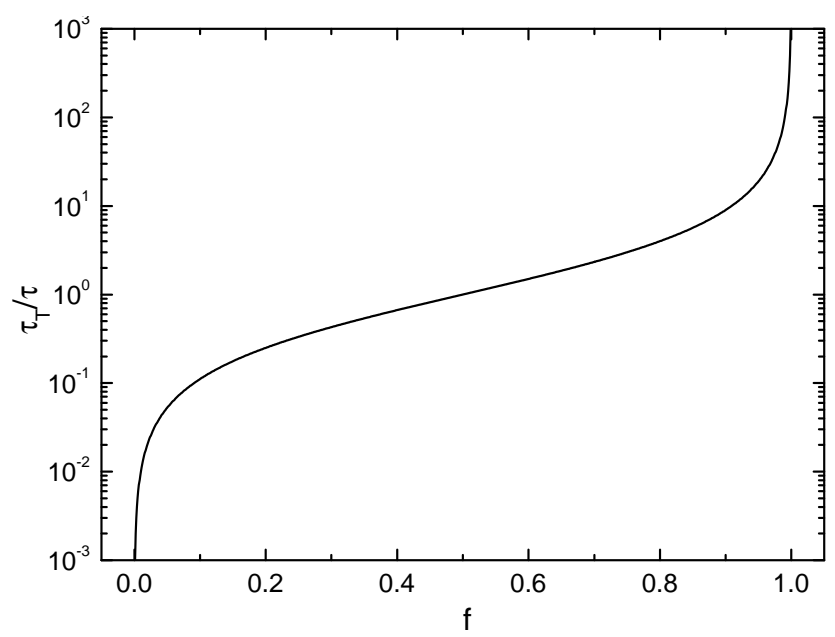

Figure 19: Evolution of the ratio $\tau_{T} / \tau$ as a function of the parameter $f$, which represents the spectator fraction of the decay.

$$
\frac{\tau_{T}}{\tau}=\frac{f}{1-f}
$$

where $f$ represents the spectator fraction of the decay ${ }^{214}$

$$
f=\frac{I_{2 h 1 e}}{I_{2 h 1 e}+I_{2 h}}=\frac{I_{2 h 1 e}}{I_{\text {total }}} .
$$

Due to the steep increase of $\tau_{T} / \tau$ for small and large values of $f$, it is reasonable to conclude that the core-hole clock method is most accurate for transfer times not too different from the core-hole lifetime.

From the experimental point of view, the situation can be more complex since there are several different decay channels, with different binding energies, which determine the formation of many overlapping spectral features. In order to discriminate between spectator Auger and normal Auger decay it is possible to rely on another difference between these two channels, besides the different electron kinetic energy. The spectator-type channels disperse with photon energy, that is the kinetic energy of the outgoing electron increases linearly with the energy of the incoming photons. In contrast, in the case of the normal Auger channels, the kinetic energy of the outgoing electron remains constant. ${ }^{219,222}$ Experimentally 
this measurement is possible by using exciting radiation with bandwidth below the natural linewidth of the core-excited state. The different dependence of the two Auger channels on the energy of the exciting photons can be understood as follows. In the case of electron transfer, the kinetic energy of the outgoing electron is constant with photon energy because the transfer process takes with it the information about the exact excitation energy so that the energy information is lost. ${ }^{219}$ If the excited electron remains localized during core-hole decay, the kinetic energy of the outgoing electron increases linearly with photon energy since the binding energy remains constant. In Sect. 6.1 a few applications of core-hole spectroscopy will be reviewed showing sub-femtosecond temporal resolution.

\subsubsection{Attoclock}

In Sect. 3.1.2 we have seen that the attosecond streaking technique allows one to map time to photo-electron energy. A very different approach is based on attosecond angular streaking: this technique is called attoclock. ${ }^{18,223,224}$ In this case, a femtosecond laser pulse with almost circular polarization is used to ionize an atom. The freed electrons are subsequently accelerated and deflected by the rotating laser field in the angular spatial direction: in this way the instant of ionization is mapped to the final angle of the momentum vector. In close analogy with the three-step model of HHG, the physical processes at the heart of the attoclock technique can be divided into two steps. The first one is tunnel ionization. In the second one, the electron, with zero initial kinetic energy, is accelerated in the radial and angular direction by the rotating electric field of the laser pulse. For pulses with less than two optical cycles ( $<6$ fs at $800-n m$ central wavelength), tunnel ionization is confined to the central optical cycle: in this case, the time-to-angle mapping is completely univocal. ${ }^{225}$ For perfectly circularly polarized pulses, one degree of angular resolution corresponds to 7.4 as at a central wavelength of $800 \mathrm{~nm}$, since the peak of the driving electric field rotates by $360^{\circ}$ within an optical cycle. Therefore, by measuring the final momentum vector with an angular accuracy of $\Delta \theta \approx 1^{\circ}$ it is possible to obtain a temporal resolution in the attosecond temporal 
domain, without employing attosecond pulses. By using theoretical models, the ultimate temporal resolution of the attoclock technique has been estimated to be around 200 as. ${ }^{223}$ Independent measurements have to be performed to characterize the temporal evolution of the driving electric field: this is required to obtain a correct interpretation of the experimental results. Indeed, the radial and transverse components of the final momentum vector depend on various pulse characteristics: the ellipticity, the peak intensity, the field envelope and the CEP. In particular, in the case of few-optical-cycle pulses with circular polarization, CEP determines the angular position of the maximum electric field vector, as shown in Fig. 20. If the CEP is varied by $\Delta \phi$, the pulse electric field is rotated in space by $\Delta \phi$. Since most of the tunnel ionization is produced near the maximum of the electric field, the CEP controls the direction of electron emission in the polarization plane.
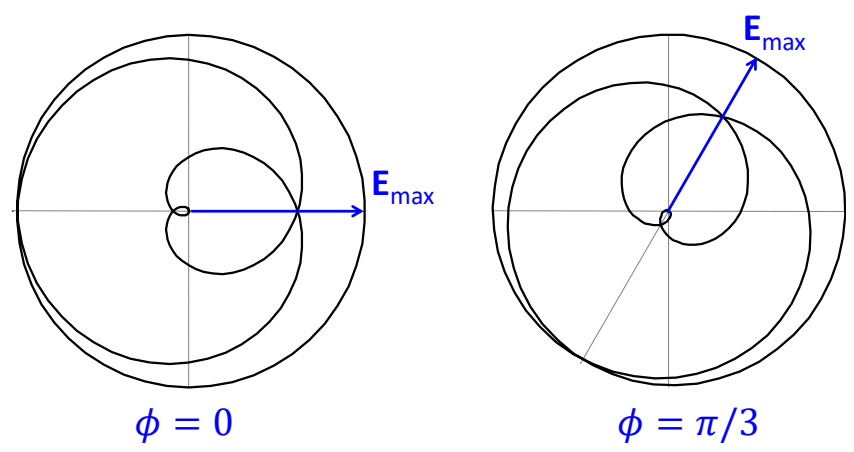

Figure 20: Spatial evolution of the electric field of a circularly polarized pulse for two different values of the CEP. As shown, a CEP-shift $\Delta \phi$ leads to a rotation in space of the electric field by $\Delta \phi$.

In the case of elliptically polarized pulses, the electric field amplitude changes with time following the polarization ellipse, so that within a single optical cycle it presents two local maxima, when the electric field vector is oriented along the major axis of the ellipse, and two local minima, when the field vector is oriented along the minor axis. As a consequence, the angular momentum distribution presents two pronounced peaks separated by $\sim 180^{\circ}$, whose relative intensity changes with the CEP of the excitation pulses, as shown in Fig. 21, while their angular position is only weakly affected by the CEP value, since the spatial orientation of the ellipticity axis does not change with CEP. While for circular polarization the time- 

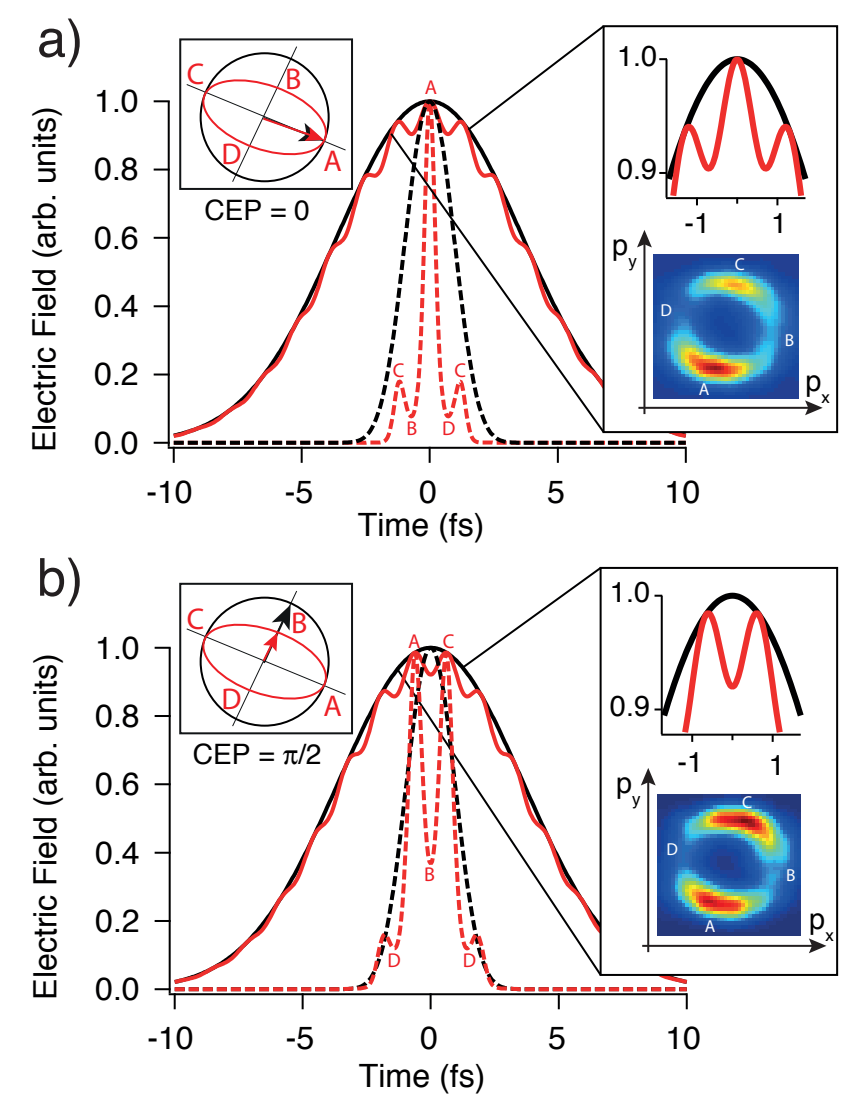

Figure 21: The solid lines show the temporal evolution of the electric field of a 5.5-fs pulse with different $\mathrm{CEP}$ values: $\mathrm{CEP}=0$ (a), $\mathrm{CEP}=\pi / 2$ (b). The dashed lines show the corresponding ionization rate. Black lines correspond to purely circularly polarized pulses, red lines correspond to elliptically polarized pulses, with $\epsilon=0.92$. The insets show a zoom around the central optical cycle and the momentum distributions $\left(p_{x}, p_{y}\right)$. Adapted from ref. 226 with permission. Copyright 2013 Springer.

dependent field envelope does not depend on CEP, in the case of elliptical polarization such envelope shows sub-cycle oscillations with a period of approximately half an optical cycle of the laser field, as shown in Fig. 21. Even a minor amount of ellipticity (which is almost unavoidable from the experimental point of view) results in a modulation of the electric field, which determines a very large variation of the ionization rate, due to the high nonlinearity of the ionization process. Indeed, a variation of the order of $10 \%$ of the electric field amplitude produces a variation of the ionization rate by almost one order of magnitude. Note that the residual ellipticity of the pulse turns out to be an advantage for the measurement, since the orientation of the ellipse provides the reference for the orientation of the electric field 
maximum, without the need for determining or locking the CEP, ${ }^{223}$ so that it offers an absolute timing for the measurements. In the case of longer pulses, where the ionization process is no longer confined to a single optical cycle, the one-to-one time-to-angle mapping is lost. However in this case the radial electron momentum can be used to obtain timing information. ${ }^{224}$

The attoclock technique has been used to set an upper limit of 34 attoseconds and an intensity-averaged upper limit of 12 attoseconds on the tunneling delay time in strong field ionization of a helium atom ${ }^{227}$ and to measure the ionization times of the two electrons in the double ionization of argon. ${ }^{224}$ In the last case, it was found that the ionization time of the first electron is in good agreement with the simulation, whereas the ionization of the second electron occurs significantly earlier than predicted. Using an attoclock setup with a COLTRIMS apparatus, ${ }^{180}$ Pfeiffer et al. demonstrated in 2012 that the tunneling time for an electron ionized from noble gas atoms is vanishingly small within the experimental accuracy of 10 as. ${ }^{18}$ Moreover they found that the freed electron presents an angular offset with respect to the expected output direction, due to the interaction with the ionic potential during the angular streaking; this offset is independent on the laser intensity for helium atoms, whereas it shows a monotonic decrease for argon ones. ${ }^{18}$ This difference has been explained in terms of multielectron and Stark shift effects, which are much more important in argon owing to its larger polarizability. Though a COLTRIMS setup allows one to record coincidence measurements suitable for a complete reconstruction of the photoelectron distribution, recent advances in this field have allowed the exploitation of Velocity Map Imaging (VMI) spectrometers for attoclock measurements. ${ }^{228}$

\subsection{X-ray Free Electron Lasers}

One of the main limitations of attosecond technology is the relatively low photon flux provided by the attosecond sources. Moreover, efficient generation of attosecond pulses in the $\mathrm{keV}$ energy range is still a great challenge. An alternative way for generating intense ul- 
trashort pulses in the VUV/X-ray energy region is provided by X-ray Free-Electron Lasers (FELs). ${ }^{229-231}$ In a FEL the active medium consists of a beam of free electrons, propagating at relativistic velocities along the axis of a spatially periodic magnet, known as an "undulator". Due to the Lorentz force, the electrons execute transverse oscillations and emit electromagnetic radiation in the forward direction. The fundamental wavelength of the emitted radiation depends on the undulation period as well as on the relativistic Lorentz factor.

In the X-ray spectral region the FEL operates as a single-pass amplifier, generating pulses with peak powers of the order of $10^{10} \mathrm{~W}$ and a pulse duration in the femtosecond range. The typical high-gain regime in which X-ray FELs operate is schematically represented in Fig. 22: electrons first enter the undulator and oscillate with random phases, thus emitting mostly incoherent radiation. Because of the collective electron interaction with the emitted radiation, the electrons begin to oscillate in phase at the resonant wavelength until the process saturates. In this regime of self-amplified spontaneous emission (SASE), ${ }^{232}$ an extremely high brightness is achieved ${ }^{233,234}$ with the drawbacks of a stochastic temporal structure and an intrinsic time jitter with respect to a measurement done in the lab frame. In recent years enormous progress has been done to overcome the inherent limitations of the SASE regime, and different seeding mechanisms have been implemented including seeding with HHG radiation ${ }^{235,236}$ (direct seeding) or seeding with a coherent bunching at the resonant wavelength. ${ }^{237,238}$ Seeding a FEL has three major advantages: i) improve the longitudinal coherence, ii) allows synchronizing the FEL radiation with a probe laser for time resolved experiments and iii) improve the shot to shot stability of the FEL power and temporal duration.

The science community immediately recognized the great potential of X-ray FELs to probe the ultrasmall (nm-resolution) and ultrafast (fs/as-resolution) worlds and a number of ground-breaking experiments have been conducted so far exploiting both SASE and seeded FELs including single-shot coherent diffractive imaging, ${ }^{239,240}$ time-resolved studies 


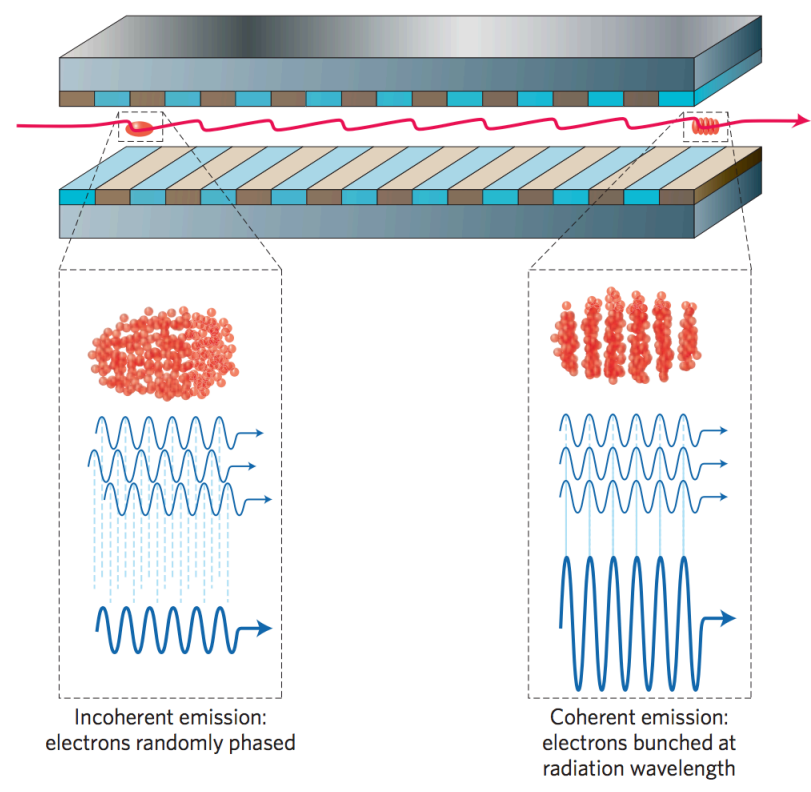

Figure 22: Schematic of the FEL operating principle: electrons first enter the undulator and oscillate with random phases, thus emitting mostly incoherent radiation. Because of the collective electron interaction with the emitted radiation, the electrons begin to oscillate in phase at the resonant wavelength until the process saturates. Reproduced with permission from ref. 230. Copyright 2010 Macmillan Publishers Limited.

in atoms, ${ }^{241}$ molecules ${ }^{242-244}$ and clusters, ${ }^{245} \mathrm{X}$-ray lasing pumped by X-ray FEL ${ }^{246}$ and controlled molecular imaging ${ }^{247}$ (for extensive review see Bostedt et al., ${ }^{248}$ Pellegrini et al. ${ }^{229}$ and Feldhaus et al. ${ }^{249}$ ). Since typical FEL pulse durations are in the order of a few tens of femtoseconds, most of the time-resolved studies reported until now were focused on the investigation of the structural dynamics of matter. However, over the past decade several methods have been proposed to generate attosecond pulses with hard X-ray FELs. Reducing $\mathrm{X}$-ray pulse durations to the attosecond regime will allow achieving both atomic-scale spatial and temporal resolution.

Recently, at the Linac Coherent Light Source, Helml et al. ${ }^{250}$ have demonstrated that a small percentage of the FEL pulses consists of individual high-intensity spikes with temporal duration of few hundreds of attoseconds. The temporal duration was measured by using nearinfrared streaking spectroscopy. The schematic of the experimental setup is shown in Fig. 23: a NIR laser pulse (streaking pulse) is coarsely synchronized to the FEL X-ray pulse and 
spatially overlapped with the X-ray beam in a Ne gas target. The photoelectrons produced by FEL photoionization are detected with a magnetic bottle electron spectrometer (MBES). When the duration of the X-ray pulses is shorter than the optical period of the streaking pulse, the photoelectron spectrum experiences a redistribution of energy. In particular the spectrum can be upshifted, downshifted or symmetrically broadened depending on the exact instant of ionization with respect to the vector potential of the streaking pulse. In contrast with attosecond streaking using HHG-based sources, time jitter does not allow the delay of the streaking pulse with respect to each single FEL pulse to be controlled. Thus, a singleshot analysis has been performed allowing for a time-to-energy mapping, and spikes with duration of 800 as have been measured. Lot of work still need to be done on the seeding of these hard X-ray lasers in order to obtain reproducible pulses, however these preliminary results already pave the way to future attosecond science using FEL sources.

\section{Theoretical methods for attosecond science}

\subsection{Molecular ionization}

As discussed in previous sections, ionization of molecules by EUV light, in particular attosecond pulses, leads in general to a coherent superposition of molecular cationic states, i.e., a non stationary electronic state or wave packet, which then evolves in atto- and fewfemtosecond time scales thus generating ultrafast electron dynamics in the molecular cation. This coherent superposition can be the result of the broad band of the ionizing pulse, which covers several ionization thresholds in the case of an attosecond pulse, or of the appearance of a dense manifold of ionic states due to rearrangements in the electronic structure of the molecular cation induced by electron correlation. In both cases the charge is expected to move from one molecular site to another or to fluctuate all along the molecular backbone.

The description of this ultrafast electron dynamics is a challenging theoretical problem. Fully $a b$ initio theoretical methods accounting for the correlated motion of electrons and 


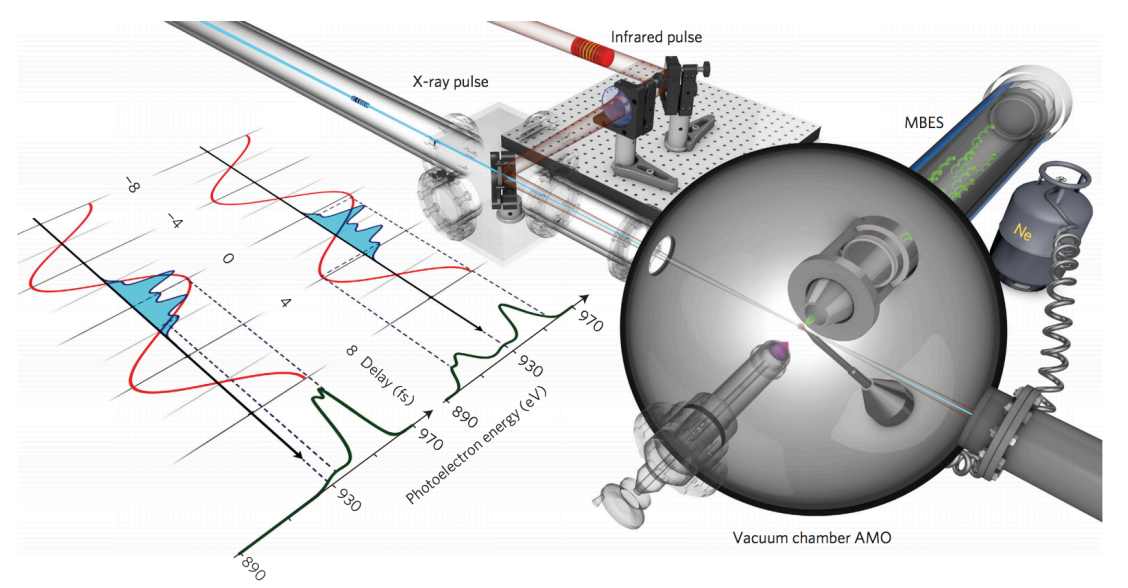

Figure 23: Scheme of the experimental set-up used at the LCLS: a NIR laser pulse (streaking pulse) is coarsely synchronized to the FEL X-ray pulse and spatially overlapped with the $\mathrm{X}$-ray beam in a Ne gas target. The photoelectrons produced by FEL photoionization are detected with a magnetic bottle electron spectrometer (MBES). Left panels illustrate the streaking principle: the X-ray spectrum is upshifted, downshifted or symmetrically broadened depending on the exact instant of ionization with respect to the vector potential of the streaking pulse. Reproduced with permission from ref. 250. Copyright 2014 Macmillan Publishers Limited.

nuclei that follows ionization are only available for diatomic molecules, mainly $\mathrm{H}_{2} \cdot{ }^{27-32}$ The reason is that, besides the intrinsic limitations due to the large number of electronic and nuclear degrees of freedom associated with polyatomic molecules (limitations that also exist in calculations involving molecular bound states), there is the additional problem of dealing with the molecular electronic continuum, which usually has a much richer structure than in atoms or diatomic molecules. Thus, most theoretical treatments of charge migration processes in large molecules have ignored the ionization step and have only described the cation dynamics that arises from arbitrarily chosen initial wave packets. For example, pioneering work on charge migration ${ }^{34,35,251}$ assumed that ionization is a sudden process that leaves a well defined hole in one of the molecular orbitals of the system. The theoretical study of the evolution of such hole led to the first reliable estimations of the time scales involved in charge 
migration. ${ }^{34,35,252,253}$ However, a correct theoretical description of the charge migration induced by attosecond pulses requires the explicit evaluation of all the ionization amplitudes that define the coherent superposition of ionic states. ${ }^{25,254}$ One can expect that the initial characteristics of this electronic wave packet, which in principle depend on the frequency, duration, and spectral phase of the attosecond pulse, will dictate the fate of the molecule, including its possible fragmentation at longer times.

In this subsection we will review the existing theoretical methods that allow one to describe the photoionization step in molecules, i.e., the onset of all charge migration processes induced by ultrashort pulses.

\subsubsection{Time-dependent molecular wave packet}

In quantum mechanics, the evolution of molecular systems is given by the solution of the time-dependent Schrödinger equation (TDSE):

$$
i \frac{\partial}{\partial t} \Phi(\mathbf{r}, \mathbf{R}, t)=\mathcal{H}(\mathbf{r}, \mathbf{R}, t) \Phi(\mathbf{r}, \mathbf{R}, t)
$$

where $\mathbf{r}$ and $\mathbf{R}$ stand for all electronic and nuclear coordinates, respectively, and $\mathcal{H}(\mathbf{r}, \mathbf{R}, t)$ is the total Hamiltonian operator that, in the presence of an external electromagnetic field, can be written as the sum of the Hamiltonian of the field-free molecule, $\mathcal{H}_{0}(\mathbf{r}, \mathbf{R})$, and the light-molecule interaction potential, $V(\mathbf{r}, t)$,

$$
\mathcal{H}(\mathbf{r}, \mathbf{R}, t)=\mathcal{H}_{0}(\mathbf{r}, \mathbf{R})+V(\mathbf{r}, t)
$$

In general, the time-dependent molecular wave packet, $\Phi(\mathbf{r}, \mathbf{R}, t)$, resulting from ionization by a pulse of duration $T$ can be written as a superposition of molecular eigenstates,

$$
\Phi(\mathbf{r}, \mathbf{R}, t>T)=\sum_{n} c_{n} e^{-i E_{n} t} \Psi_{n}(\mathbf{r}, \mathbf{R})+\sum_{E_{f}} c_{E_{f}} \mathrm{e}^{-i E_{f} t} \Psi_{E_{f}}(\mathbf{r}, \mathbf{R})
$$


where $\Psi_{n}(\mathbf{r}, \mathbf{R})$ are the bound states of the neutral molecule with energy $E_{n}$ and $\Psi_{E_{f}}(\mathbf{r}, \mathbf{R})$ are electronic continuum states with energy $E_{f}$, which is shared between the ejected electron and the molecular ion left behind. The coefficients $c_{n}$ and $c_{E_{f}}$ are thus the excitation and ionization amplitudes after the interaction with the external field. The field-free evolution after the ionization event is simply given by the $E_{j} t$ phases, since the above expansion is written in terms of the eigenstates of the system, which are the solutions of the eigenvalue problem

$$
\mathcal{H}_{0}(\mathbf{r}, \mathbf{R}) \Psi_{j}(\mathbf{r}, \mathbf{R})=E_{j} \Psi_{j}(\mathbf{r}, \mathbf{R})
$$

where the field-free Hamiltonian for a molecule containing $M$ nuclei with $M_{I}$ masses and $N$ electrons with $m_{e}=1$ mass is given in atomic units by

$$
\mathcal{H}_{0}(\mathbf{r}, \mathbf{R})=-\sum_{I=1}^{M} \frac{1}{2 M_{I}} \nabla_{\mathbf{R}_{I}}^{2}-\sum_{i=1}^{N} \frac{1}{2} \nabla_{i}^{2}+\sum_{i=1}^{N} \sum_{i>j} \frac{1}{\left|\mathbf{r}_{i}-\mathbf{r}_{j}\right|}-\sum_{i=1}^{N} \sum_{I=1}^{M} \frac{Z_{I}}{\left|\mathbf{r}_{i}-\mathbf{R}_{I}\right|}+\sum_{I=1}^{M} \sum_{J>I} \frac{Z_{I} Z_{J}}{\left|\mathbf{R}_{I}-\mathbf{R}_{J}\right|}
$$

In the latter equation, mass polarization and relativistic effects have been neglected. Molecular rotation terms are usually ignored because rotation is orders of magnitude slower (picoseconds, $1 \mathrm{ps}=10^{-12} \mathrm{~s}$ ) than the processes we are interested in, for instance charge migration or molecular dissociation, and much slower than the laser-molecule interaction that occurs within the femtosecond/sub-femtosecond time scales. Note that the expansion given in eq 35 assumes that bound and continuum wave functions are normalized to unity. This normalization arises naturally when eq 36 is solved in a finite region of space, leading to a discretization of the electronic and vibrational continua. The correct normalization of continuum states to the Dirac delta, $\delta\left(E_{f}-E_{f}^{\prime}\right)$, can be later recovered by using the corresponding density of states. ${ }^{255}$

Inserting the expansion given by eq 35 in the TDSE defined in eq 33, one obtains a system 
of coupled linear differential equations that can be written in matrix-vector form as

$$
i \frac{\partial}{\partial t}\left(\begin{array}{c}
\mathbf{c}_{n} \\
\mathbf{c}_{E_{f}}
\end{array}\right)=\left(\begin{array}{cc}
\mathbf{V}(t)_{n}^{n^{\prime}} & \mathbf{V}(t)_{n}^{E_{f}} \\
\mathbf{V}(t)_{E_{f}}^{n^{\prime}} & \mathbf{V}(t)_{E_{f}}^{E_{f}^{\prime}}
\end{array}\right)\left(\begin{array}{c}
\mathbf{c}_{n} \\
\mathbf{c}_{E_{f}}
\end{array}\right)
$$

This system of equations can be solved using standard integration schemes (Runge-Kutta, Crank-Nicolson, Lanczos, etc.). However, attosecond pulses currently generated in the laboratory are weak, with intensities typically in the range $<10^{11}-10^{12} \mathrm{~W} / \mathrm{cm}^{2}$. Under this circumstance, the relevant states involved in the molecular photoionization process are the ground state of the molecule, $\Psi_{0}(\mathbf{r}, \mathbf{R})$, i.e. the first bound state of the neutral, $n=0$, and the electronic continuum states. The ionization amplitudes can thus be obtained by using first order time-dependent perturbation theory, in which $c_{n=0} \approx 1$ and the $c_{E_{f}}$ coefficients are given by

$$
c_{E_{f}}=-i\left\langle\Psi_{E_{f}}(\mathbf{r}, \mathbf{R})\left|\boldsymbol{\epsilon} \cdot \sum_{k} \mathbf{d}_{k}\right| \Psi_{0}(\mathbf{r}, \mathbf{R})\right\rangle \int_{-\infty}^{\infty} \mathcal{E}(t) e^{i\left(E_{f}-E_{0}\right) t} d t
$$

where $\sum_{k} \mathbf{d}_{k}$ is the multielectronic dipole operator, $\boldsymbol{\epsilon}$ the polarization direction of the incident light, $\mathcal{E}(t)$ is the electromagnetic field and $\left(E_{f}-E_{0}\right)$ the energy absorbed by the system in the transition from the ground state $\Psi_{0}(\mathbf{r}, \mathbf{R})$ to the final state $\Psi_{E_{f}}(\mathbf{r}, \mathbf{R})$. An accurate evaluation of this dipole transition element is a challenging task still nowadays and very few theoretical groups have tackled the scattering problem including the proper boundary conditions. ${ }^{256-266}$ All those works have made use of the fixed nuclei approximation during the ionization step and the subsequent evolution of the molecular wave packet, thus reducing the size of the problem to tractable proportions. Some other theoretical approaches $34,35,267,268$ simply avoid the accurate evaluation of the ionization amplitudes, substituting it by a direct projection of the ground neutral state into the cationic states, and rather focus on a more elaborate description of the time-evolving charge distribution occurring after ionization of relatively large molecules. Irrespective of the methodology employed, the visualization of the latter process is commonly achieved through the evaluation of the so-called hole density. It is 
a widely employed observable to trace in time the evolution of the positive charge created in a large molecule after prompt ionization. The hole density is defined as the difference between the one-electron densities of the ground state of the target and that of the cation, ${ }^{34,267}$

$$
\rho_{\text {hole }}\left(\mathbf{r}_{1}, t\right)=\rho_{\text {neutral }}\left(\mathbf{r}_{1}\right)-\rho_{\text {ion }}\left(\mathbf{r}_{1}, t\right)
$$

where the explicit dependence on time is that of the ionic electron density. Note that this simplified expression is meaningful as long as the nuclear positions remain unchanged, i.e., the nuclear degrees of freedom are explicitly ignored. Otherwise, the changes in time of the nuclear geometry should be explicitly accounted for before defining the electron densities ${ }^{269}$ and the charge fluctuations would be better defined around the moving nuclear centers. The one-electron density is defined as the integral of the squared modulus of the full wave function over the space coordinates of $M$ nuclei and $N$ electrons but one, thus giving:

$$
\rho\left(\mathbf{r}_{1}\right)=N \int d \mathbf{r}_{2} \ldots \int d \mathbf{r}_{N} \int d \mathbf{R}_{1} \ldots \int d \mathbf{R}_{M}\left|\Phi^{N}\left(\mathbf{r}_{1}, \ldots, \mathbf{r}_{N}, \mathbf{R}_{1} \ldots, \mathbf{R}_{M}\right)\right|^{2}
$$

$\rho_{\text {neutral }}$ is thus obtained from the ground state wave function $\Psi_{0}(\mathbf{r}, \mathbf{R})$ and $\rho_{\text {ion }}(\mathbf{r}, t)$ from the time-dependent wave packet $\Phi(\mathbf{r}, \mathbf{R}, t)$ defined in equation 35; always assuming the normalization of the full wave function to unity. As we have mentioned, in most implemented approaches, within the fixed nuclei approximation, the nuclear coordinates drop from the above expression and the one-electron density is writen as,

$$
\rho\left(\mathbf{r}_{1}\right)=N \int d \mathbf{r}_{2} \ldots \int d \mathbf{r}_{N}\left|\Phi^{N}\left(\mathbf{r}_{1}, \ldots, \mathbf{r}_{N}\right)\right|^{2}
$$

We dedicate the rest of this subsection to discuss the extant approaches to evaluate the molecular electronic continuum functions $\Psi_{E_{f}}\left(\mathbf{r}_{1}, \ldots, \mathbf{r}_{N}\right)$, which is in itself a challenge even for the smallest molecules. In subsection 4.2, we discuss the methods that have been used to study the charge migration that follows the ionization process, including those ones 
that avoid the explicit evaluation of the ionization amplitudes. Subsection 4.3 reviews the state-of-the-art techniques to account for nuclear motion to obtain the full dimensional wave function, $\Psi_{E_{f}}\left(\mathbf{r}_{1}, \ldots, \mathbf{r}_{N}, \mathbf{R}_{1} \ldots, \mathbf{R}_{M}\right)$. Finally, we conclude with the expressions employed to explore HHG from molecules when exposed to strong fields. Readers not interested in the details of the methods that have been developed to evaluate molecular electronic continuum states or molecular high-harmonic generation spectra can go directly to section 4.2 without loss of continuity.

\subsubsection{Electronic continuum states}

The theory of molecular continuum states reached in photoionization is well known. ${ }^{270-272}$ In the following we will review the available methods to describe molecular photoionization at fixed geometry, i.e., we will drop all reference to nuclear coordinates $\mathbf{R}$.

The most general formulation is obtained via a close-coupling (CC) expansion, where the molecular continuum states can be written as ( $N$ electrons)

$\Psi_{E_{\alpha} l m}^{N}\left(\mathbf{r}_{1}, \ldots, \mathbf{r}_{N}\right)=\sum_{\alpha^{\prime} l^{\prime} m^{\prime}} \mathcal{A}\left[\Psi_{\alpha^{\prime}}^{N-1}\left(\mathbf{r}_{1}, \ldots, \mathbf{r}_{N-1}\right) \varphi_{\varepsilon_{\alpha}, \alpha l m, \alpha^{\prime} l^{\prime} m^{\prime}}\left(\mathbf{r}_{N}\right)\right]+\sum_{K} C_{E_{\alpha} l m, K} \Phi_{K}^{N}\left(\mathbf{r}_{1}, \ldots, \mathbf{r}_{N}\right)$

where $\alpha, \alpha^{\prime}$ are quantum numbers necessary to identify the ionic states $\Psi_{\alpha^{\prime}}^{N-1}$ included in the expansion to obtain the corresponding continuum eigenstates $\Psi_{E_{\alpha} l m}^{N}$ with total energy $E_{\alpha}$, and $\varphi_{\varepsilon_{\alpha} \alpha l m, \alpha^{\prime} l^{\prime} m^{\prime}}\left(\mathbf{r}_{N}\right)$ is the one-electron continuum function for a photoelectron ejected with a set of angular quantum numbers $l m$ and with $\varepsilon_{\alpha}$ energy, leaving behind the $\alpha$ ionic state. The continuum functions must satisfy the proper asymptotic conditions of scattering states, ${ }^{272-274}$ i.e.,

$$
\varphi_{\varepsilon_{\alpha} \alpha l m, \alpha^{\prime} l^{\prime} m^{\prime}}(\mathbf{r}) \underset{\mathbf{r} \rightarrow \infty}{\longrightarrow}\left[f_{l^{\prime}}\left(k_{\alpha^{\prime}} r\right) \delta_{l l^{\prime}} \delta_{m m^{\prime}} \delta_{\alpha \alpha^{\prime}}+g_{l^{\prime}}\left(k_{\alpha^{\prime}} r\right) K_{\alpha l m, \alpha^{\prime} l^{\prime} m^{\prime}}\right] Y_{l^{\prime} m^{\prime}}(\theta, \phi)
$$

where we have chosen $K$-matrix boundary conditions, which have the advantage of working with real functions. Here $f_{l^{\prime}}\left(k_{\alpha^{\prime}} r\right)$ and $g_{l^{\prime}}\left(k_{\alpha^{\prime}} r\right)$ are the regular and irregular asymptotic 
functions (Coulomb functions in the present case), and $k_{\alpha^{\prime}}$ is the electron momentum relative to ionization leaving the cation in the $\Psi_{\alpha^{\prime}}^{N-1}$ state. The square integrable configurations $\Phi_{K}^{N}$ in eq 43 take care of the short range correlations. $\mathcal{A}$ implies antisymmetrization and proper symmetry coupling.

It is easy to transform wave functions $\Psi_{E_{\alpha} l m}^{N}$ or dipole matrix elements

$$
\mathbf{D}_{E_{\alpha} l m}=\left\langle\Psi_{E_{\alpha} l m}^{N}\left|\sum_{k} \mathbf{d}_{k}\right| \Psi_{0}^{N}\right\rangle
$$

to the usual incoming wave boundary conditions as follows

$$
\boldsymbol{\Psi}_{E_{\alpha} l m}^{(-)}=(\mathbf{1}+i \mathbf{K})^{-1} \Psi_{E_{\alpha} l m}^{N}
$$

where $\Psi_{E_{\alpha} l m}^{(-)}$behaves asymptotically as a superposition of outgoing waves in all channels $\alpha^{\prime}$ and partial waves $l^{\prime}$, and an incoming wave in channel $\alpha$ and partial wave $l$. In principle, the CC expansion can be very accurate and can be considered as a generalization of the Configuration Interaction (CI) approach to the continuum. In practice, it is very demanding and it is generally limited to rather small systems. As focus shifts to larger molecules, strong simplifications are required, generating a series of more approximate approaches.

\subsubsection{Single Channel Approximation}

Restricting the CC expansion in eq 43 to a single term, the molecular continuum states can be simplified as

$$
\Psi_{E_{\alpha} l m}^{N}\left(\mathbf{r}_{1}, \ldots, \mathbf{r}_{N}\right)=\mathcal{A}\left[\Psi_{\alpha}^{N-1}\left(\mathbf{r}_{1}, \ldots, \mathbf{r}_{N-1}\right) \varphi_{\varepsilon_{\alpha} l m}\left(\mathbf{r}_{N}\right)\right]
$$


which is a more tractable approach. Here a prominent role is played by the Dyson orbital defined as 275,276

$$
\varphi_{\alpha}^{D}(\mathbf{r})=N \int \Psi_{\alpha}^{N-1 \star}\left(\mathbf{r}_{1}, \ldots, \mathbf{r}_{N-1}\right) \Psi_{0}^{N}\left(\mathbf{r}, \mathbf{r}_{1}, \ldots, \mathbf{r}_{N-1}\right) d \mathbf{r}_{1} \ldots d \mathbf{r}_{N-1}
$$

which represents the overlap between the $N$-electron ground state wave function of the neutral molecule $\Psi_{0}^{N}$ and the $(N-1)$-electron wave function of the $\alpha$ state of the molecular cation $\Psi_{\alpha}^{N-1}$.

Neglecting for the moment the actual evaluation of the continuum orbital $\varphi_{\varepsilon_{\alpha} l m}$, the calculation of the dipole transition moments

$$
\mathbf{D}_{E_{\alpha} l m}=\left\langle\mathcal{A}\left[\Psi_{\alpha}^{N-1} \varphi_{\varepsilon_{\alpha} l m}\right]\left|\sum_{k} \mathbf{d}_{k}\right| \Psi_{0}^{N}\right\rangle=\left\langle\varphi_{\varepsilon_{\alpha} l m}|\mathbf{d}| \varphi_{\alpha}^{D}\right\rangle+\left\langle\varphi_{\varepsilon_{\alpha} l m} \mid \eta_{\alpha}^{D}\right\rangle
$$

reduces to single particle transition matrix elements, involving the Dyson orbital $\varphi_{\alpha}^{D}$ and the "conjugate" Dyson orbital $\eta_{\alpha}^{D}$, which are computed entirely from the bound state wavefunctions, $\Psi_{\alpha}^{N-1}$ and $\Psi_{0}^{N} \cdot{ }^{277-279}$ The first term represents a dipole transition between the Dyson orbital and the continuum orbital, and the second one represents a monopole transition from $\eta_{\alpha}^{D}$ to the continuum, and is often negligible, although it may become important close to threshold. In terms of an orbital basis $\left\{\varphi_{p}\right\}$, and the associated creation $a_{p}^{+}$and annihilation $a_{p}$ operators, which add or remove a particle in orbital $\varphi_{p},{ }^{276}$ it is easy to compute the Dyson (or spectral) amplitudes

$$
\begin{gathered}
\chi_{\alpha p}=\left\langle\Psi_{\alpha}^{N-1}\left|a_{p}\right| \Psi_{0}^{N}\right\rangle \\
\xi_{\alpha p}=\left\langle\Psi_{\alpha}^{N-1}\left|\left(\sum_{k} \mathbf{d}_{k}\right) a_{p}\right| \Psi_{0}^{N}\right\rangle
\end{gathered}
$$

from which one can expand

$$
\begin{aligned}
& \varphi_{\alpha}^{D}(\mathbf{r})=\sum_{p} \chi_{\alpha p} \varphi_{p}(\mathbf{r}) \\
& \eta_{\alpha}^{D}(\mathbf{r})=\sum_{p} \xi_{\alpha p} \varphi_{p}(\mathbf{r})
\end{aligned}
$$


and

$$
\mathbf{D}_{E_{\alpha} l m}=\sum_{p} \chi_{\alpha p}\left\langle\varphi_{\varepsilon_{\alpha} l m}|\mathbf{d}| \varphi_{p}\right\rangle+\sum_{p} \xi_{\alpha p}\left\langle\varphi_{\varepsilon_{\alpha} l m} \mid \varphi_{p}\right\rangle
$$

Physically the first term describes dipole transitions from initial orbitals $\varphi_{p}$ to the continuum orbital, multiplied by an overlap between the ionic state $\Psi_{\alpha}^{N-1}$ and the initial state with an electron annihilated in $\varphi_{p}$; the second term describes a monopole transition from $\varphi_{p}$ to the continuum, multiplied by a dipole transition between the remaining bound states. ${ }^{277,278}$ We shall neglect the second term from now on. Dyson orbitals have become widely employed in recent work, ${ }^{280,281}$ notably for strong field or time resolved photoionization. ${ }^{282,283}$

The situation becomes very transparent if we consider the initial state

$$
\Psi_{0}^{N}=\Phi_{0}=\left|\varphi_{1}, \ldots, \varphi_{N}\right\rangle
$$

described as a single determinant (e.g. at the Hartree-Fock (HF) level), and $\Psi_{\alpha}^{N-1}$ described as single configurations built from the same set of orbitals. Then clearly $\chi_{\alpha k}=1$ for the single-hole configurations

$$
\Psi_{\alpha}^{N-1}=\Phi_{k}=a_{k} \Phi_{0}
$$

called primary states, and $\chi_{\alpha p}=0$ otherwise. We shall call this description the Koopmans' picture. Correlation effects redistribute intensity towards other states, and will be discussed later.

\subsubsection{Continuum Orbitals}

In the case of primary ionizations described as single determinants in eq 53 , one has

$$
\Psi_{E_{\alpha} l m}^{N}=a_{\varepsilon_{k} l m}^{+} a_{k} \Phi_{0}=\left|\varphi_{1}, \ldots, \varphi_{k-1} \varphi_{\varepsilon_{k} l m} \ldots \varphi_{N}\right\rangle
$$

i.e. a single excitation from the occupied orbital $\varphi_{k}$ to the continuum one $\varphi_{\varepsilon_{k}} l m$, again in single determinant form, neglecting spin and symmetry couplings that can be easily taken 
into account. The continuum orbital is the solution of a single particle equation

$$
h_{k} \varphi_{\varepsilon_{k} l m}(\mathbf{r})=\varepsilon_{k} \varphi_{\varepsilon_{k} l m}(\mathbf{r}) .
$$

At the ab initio level, $h$ becomes an appropriate Fock-type operator, and the approach is called "static-exchange" or just continuum HF. ${ }^{284,285}$ Alternatively, one can employ density functional theory (DFT) for the description of the ground state, and build $\Phi_{0}$ from the lowest Kohn-Sham (KS) orbitals, which are the solutions of the eigenvalue equations ${ }^{286}$

$$
\begin{gathered}
h_{K S} \varphi_{k}(\mathbf{r})=\varepsilon_{k} \varphi_{k}(\mathbf{r}), \\
h_{K S}=-\frac{1}{2} \nabla^{2}+V_{n u c}+V_{C}+V_{X C},
\end{gathered}
$$

where $V_{\text {nuc }}$ is the nuclear attraction term, $V_{C}(\rho)$ is the Coulomb potential generated by the ground state density $\rho(\mathbf{r})$, and $V_{X C}(\rho)$ is the exchange-correlation potential expressed in terms of the same density. Then the DFT "static-exchange", analogous to the HF one, amounts to computing the continuum solutions of the same KS hamiltonian given in eq $55:^{287}$

$$
h_{K S} \varphi_{\varepsilon l m}(\mathbf{r})=\varepsilon_{k} \varphi_{\varepsilon_{k} l m}(\mathbf{r}) .
$$

In the case of a local $V_{X C}$ potential this reduces to potential scattering, and is computationally tractable even for rather large systems.

Also in the case of a more complex, correlated description of the ionic state $\Psi_{\alpha}^{N-1}$, the corresponding continuum is obtained as the solution of a single particle equation. The corresponding operator is more complex, but can be more easily expressed as a hamiltonian matrix in a basis set approach, like in the full CC case. The approach can be further simplified by employing a more approximate potential for the determination of $\varphi_{\varepsilon_{\alpha} l m}$. For instance, the exchange-correlation potential defined by the first order reduced density matrix $\rho_{\alpha}\left(\mathbf{r} ; \mathbf{r}^{\prime}\right)$ relative to $\Psi_{\alpha}^{N-1}$. Even more simply, employing the HF or DFT static-exchange continuum 
in eq 51 is still deemed a rather good approximation. In the case of DFT, we shall call it DFT-Dyson approach.

\subsubsection{Computational Approaches to Continuum Orbitals}

One can neglect any coupling with the ionic state altogether, and employ a simple Coulomb wave (CW) or just a Plane wave (PW) for the calculation of transition dipole matrices. A variant includes orthogonalization of the $\mathrm{PW}(\mathrm{OPW})$ or the $\mathrm{CW}(\mathrm{OCW})$ to the initial orbital. That can be easily achieved by choosing the origin as to annihilate the dipole moment $\left\langle\varphi_{\alpha}^{D}|d| \varphi_{\alpha}^{D}\right\rangle$ of the initial orbital. ${ }^{283}$ These approaches have been tested in the early days of photoelectron spectroscopy, ${ }^{288-291}$ but then abandoned as they generally produced rather poor results, and better approaches became soon available. They have been recently revived ${ }^{292}$ essentially because of the need to compute dipole matrix elements in the context of more elaborate calculations, like time resolved photoelectron spectroscopy (TRPES) ${ }^{283}$ or close coupling approach to the charge migration problem. ${ }^{293-295}$ A recent comparison with the DFT-Dyson approach on furan has confirmed, however, the rather poor performance of such approaches, and the relative minor role of orthogonalization. ${ }^{296}$

Even the solution of single particle continuum equation is not trivial for a polyatomic molecule, due to the Coulomb singularities at each nucleus. Basically, all approaches employ large one-center expansions (OCE), generally supplemented by a restricted set of atomcentered basis functions, in the spirit of the linear combination of atomic orbitals (LCAO) approach for bound states. The pure OCE approach

$$
\varphi_{\varepsilon l m}(r, \theta, \phi)=\sum_{l^{\prime} m^{\prime}} R_{\varepsilon l m, l^{\prime} m^{\prime}}(r) Y_{l^{\prime} m^{\prime}}(\theta, \phi)
$$

where the wave function is expanded in products of radial wave functions $R(r)$ and spherical harmonics $Y_{l m}(\theta, \phi)$, is straightforward, as it reduces to a set of coupled ordinary differential equations that can be easily integrated, or expanded in a basis, and is still employed in some 
applications. ${ }^{271,297}$ It covers a long range expansion, typically some tens of atomic units or more, to reach the asymptotic region where the solution is fitted to its analytic asymptotic form. It is very stable numerically, but it converges very slowly with angular momenta, the more so the farther the atoms are from the expansion center, and it becomes quickly impractical for large low-symmetry systems. A basis set expansion of the long range part requires special functions, as the pure gaussian (GTO) or Slater (STO) type orbitals of bound state calculations are inherently incapable of accurately describing the oscillatory nature of the continuum orbitals at large distance. Convergence becomes very fast by supplementing OCE, which is in any case needed to impose the asymptotic boundary conditions, with LCAO type functions centered at the nuclei. These are of $L^{2}$-integrable type, either conventional GTO functions or functions like those employed in the OCE expansion.

Finally, continuum solutions do not satisfy the usual Raleigh-Ritz variational principle currently employed in bound state calculations. Either scattering type variational approaches (Complex Kohn, ${ }^{298}$ Schwinger ${ }^{299,300}$ ), Lippmann-Schwinger, ${ }^{255,301}$ R-matrix ${ }^{271}$ or Galerkin (Least Squares) ${ }^{302-305}$ approaches can be employed. Despite technical differences all algorithms work equivalently, and the accuracy of the solution is basically determined by the quality of the basis set employed.

Alternative approaches include the Continuum Multiple-Scattering (CMS) method, and grid-based methods. CMS 306 introduces a further approximation to the potential, the muffin tin (MT) approximation, which partitions space into spherical regions around the nuclei and the outer region, and an interstitial region where the potential is assumed constant. Although abandoned for bound state calculations, where the approximation turns out to be quite severe, it is still employed with reasonable accuracy for the continuum. ${ }^{307,308}$ Grid methods in cartesian coordinates suffer from poor description of the regions around the nuclei, where pseudo potentials are often used, and the difficulty of implementing asymptotic boundary conditions at long range. Adaptive grid methods have been developed, ${ }^{309}$ which surmount these difficulties, but tend to give less accurate representations of the laplacian. 
These methods are computationally effective but, at variance with basis set approaches, cannot be easily implemented beyond a pure local potential approach, or in the general CC environment.

\subsubsection{Coupled Channel approaches}

The simplest coupled channel approach is the use of the single excitation CI approach (CIS, Configuration Interation Singles, also called 1h1p -for one hole one particle- CI, or TammDancoff approximation, TDA), where only the primary ionic channels, at the single determinant level (54) are coupled. Despite its simplicity, this approach is attractive because of the ease of implementation and the fact that it describes the dominant interaction between primary channels, as is well known from bound state excitations. It has been recently employed in the atomic case ${ }^{310-313}$ and for molecular applications to total ionization probabilities. ${ }^{314-317}$ A B-spline implementation for the continuum has been recently developed. ${ }^{318}$ A relatively simple generalization, in the context of linear response, or propagator theory, is the Random Phase Approximation (RPA), and its DFT counterpart, time-dependent DFT (TDDFT), which often provides better transition dipoles due to an improved treatment of ground state correlation. Both have been extensively employed in the context of molecular photoionization, ${ }^{319,320}$ TDDFT being applicable also to pretty large systems. ${ }^{265,321,322}$

Implementations of the full $\mathrm{CC}$ expansion are available in several groups for the treatment of electron scattering and photoionization. ${ }^{271,272}$ Among them are those based on the Schwinger ${ }^{256,257,300}$ or complex Kohn ${ }^{298}$ variational approaches, the molecular R-matrix code, ${ }^{323}$ and the Lippmann-Schwinger formalism, ${ }^{28,255,301}$ with newer codes being actively developed in several laboratories. ${ }^{305,324-328}$ They are all based on a Quantum Chemistry description of the bound states, $\Psi_{0}^{N}$ and $\Psi_{\alpha}^{N-1}$, as CI expansions, and basis sets $\chi_{\mu}$ generally based on finite elements or B-splines for the long range continuum, although GTOs are also employed for the full inner R-matrix region in the UK R-matrix code ${ }^{323}$ or for the description of the continuum state in the short range region. ${ }^{305,328,329}$ The major computational 
bottlenecks are the construction of the hamiltonian matrix elements, both due to the close coupling form of the basis, of the product form $\Psi_{\alpha}^{N-1} \chi_{\mu}$, and the unwieldy two electron matrix elements between molecular orbitals, MO's, generally expressed in GTOs and the special functions employed for the description of the continuum. Often large OCE expansions are employed as intermediate steps. Also orthogonality constraints required to assure uniqueness of the solutions are an issue. We refer to the recent literature for examples of current capabilities. ${ }^{262,266,330,331}$

Finally, a time dependent close-coupling approach, based on eq 35 and eq 38 , with a discretization of continuum, as well as double continuum, in plane waves (OPW) has been presented and applied in Ref. 294.

\subsubsection{The B-spline static exchange DFT method}

The B-spline code built in the framework of the static exchange DFT method is the only one that has been actually employed in the evaluation of the initial electronic wave packet created by an attoseond pulse in large molecules, which, as mentioned above, requires the evaluation of transition dipole elements for a correct description of charge migration in those systems.

The core of the program is based on a multicentre $B$-spline basis. ${ }^{264} \mathrm{~B}$-splines are a set of piecewise polynomial functions defined over a given interval, $\left[0, R_{\max }\right]$ in the present case, divided in subintervals by a grid of knots. ${ }^{255,332}$ They are completely defined by the polynomial order (usually 10) and the knot sequence, and can approximate arbitrarily well both bound and continuum orbitals.

Primitive basis functions are products of a $B$-spline radial function times a real spherical harmonic

$$
\chi_{i l m}(r, \theta, \phi)=\frac{1}{r} B_{i}(r) Y_{l m}(\theta, \phi) .
$$

The full basis comprises a large OCE expansion on a common origin, with long range $R_{\max 0}$, and large maximum angular momentum, $L_{\max 0}$. This is supplemented by additional func- 
tions centered on the nuclei, of very short range, $R_{\operatorname{maxp}}$, and small angular momenta $L_{\operatorname{maxp}}$. A short range is necessary to avoid (quasi) linear dependence of the basis, which spoils the numerical stability of the approach. $L_{\operatorname{maxp}}$ is set as in conventional LCAO calculations, e.g. the maximum $l$ occupied in the atom plus one. Despite the very limited number of LCAO functions, these choices ensure a very fast convergence of the calculated quantities. For the same reason, the computational cost is entirely determined by the size of the OCE.

The calculation of continuum eigenvectors is performed at any selected electron kinetic energy by using the Galerkin approach originally proposed in Ref. 302 and generalized to the multichannel case. ${ }^{303,304}$ From the energy independent hamiltonian $H$ and overlap $S$ matrices, continuum vectors are obtained as eigenvectors of the energy dependent matrix $A(E)=H-E S$ with eigenvalues very close to zero. These give the correct number of independent one-channel solutions, and are efficiently obtained by block inverse iteration, since they are separated by large gaps from the rest of the spectrum. Actually, the more stable form $A^{+} A$ is currently employed. ${ }^{333}$ Final normalization to $K$-matrix boundary conditions is obtained by fitting the solutions to the analytical asymptotic form at the outer boundary $R_{\max 0}$

As an example, in the recently studied case of phenylalanine, ${ }^{25,254}$ the parameters employed are: $R_{\max 0}=30.0$ a.u., linear grid with step size $h=0.375$ a.u. thus giving 108 B-splines, $L_{\max 0}=21, R_{\operatorname{maxp}}=0.71-1.30$ a.u., and $L_{\max }=2$ for all atoms.

The basic algorithm has been generalized to linear response TDDFT, employing a stable non iterative algorithm, with $V^{S C F}$ as the basic dynamical variable, which is expanded in the same basis, avoiding the increased dimensionality of $1 h-1 p$ matrices typical of Quantum Chemistry implementations. ${ }^{334}$ This allows still the treatment of rather large systems, ${ }^{321,322}$ although the computational cost is 1-2 orders of magnitude larger than that of the staticexchange DFT approach.

The static-exchange DFT approach has been applied to the calculation of photoionization observables, like cross sections, angular distributions, shape resonances, molecular-frame 
photoelectron angular distributions (MFPADS), in a large sample of molecular systems, with generally remarkable accuracy. ${ }^{322,335-337}$ The TDDFT version of this approach adds interchannel couplings, that are proved to be important to describe closed channel autoionization resonances, ${ }^{322}$ especially with heavier atoms. The DFT-Dyson formulation extends the range of application to correlated or multi-configurational ionic states, like cross sections of satellite states, or ionization from excited states. ${ }^{279,296}$

\subsection{Charge Migration}

In this chapter, we will review the most relevant theoretical approaches to describe charge migration in large molecules. These approaches can be classified in two categories. The first one includes a large number of theoretical studies ${ }^{34,35,253,338,339}$ based on the so called "sudden approximation" (SA). ${ }^{275,277,340}$ In this approximation, it is assumed that a hole is created in a specific molecular orbital of the initial wave function and that this hole has no time to relax, thus leading to an electronic wave packet (WP) in terms of the eigenfunctions of the final molecular ion. In this context, the study of charge migration processes does not require the knowledge of ionization probabilities and, for this reason, most theoretical calculations performed up to date belong to this category. These methods are reviewed in 4.2.1. However, as explained above, a broadband attosecond pulse leads to a coherent superposition of several one-hole states, which can only be correctly described by explicitly evaluating the corresponding ionization amplitudes. Methods capable of dealing with actual ionization amplitudes for the description of charge migration processes belong to the second category. These methods will be described in 4.2.2. 


\subsubsection{Methods based on the sudden approximation}

If a hole is created in an initial orbital $\varphi_{k}$, the corresponding WP is $a_{k} \Psi_{0}^{N}$, where $\Psi_{0}^{N}$ is the initial state. One can then expand

$$
a_{k} \Psi_{0}^{N}=\sum_{\alpha} \Psi_{\alpha}^{N-1}\left\langle\Psi_{\alpha}^{N-1}\left|a_{k}\right| \Psi_{0}^{N}\right\rangle=\sum_{\alpha} \chi_{\alpha k} \Psi_{\alpha}^{N-1}
$$

where the spectral amplitudes $\chi_{\alpha k}$ (see eq 48 ) can be obtained by pure bound state calculations, the most common being either Green's Function (Propagator, EOM) approaches, ${ }^{340,341}$ like the $\mathrm{ADC}(3)$ long developed by Cederbaum, Schirmer and collaborators ${ }^{342-344}$ and largely used in this context, or more recently EOM-CC or CC-LR, ${ }^{280,345,346}$ or CI approaches like SAC-CI, ${ }^{347,348}$ MRCI, CASSCF/RASSCF. ${ }^{349-352}$ At the lowest level, taking just relaxation into account by separate $\mathrm{SCF}$ calculations $(\triangle \mathrm{SCF})$ for the ground and ionic states, either at the HF or DFT level, can be used, ${ }^{35}$ where $\chi_{\alpha k}=\operatorname{det} S_{\alpha k}$, where $S_{\alpha k}$ is the overlap matrix between initial and final orbitals describing $\Psi_{\alpha}^{N-1}$ and $a_{k} \Psi_{0}^{N}$.

Analysis of correlation (relaxation) effects upon ionization has been thoroughly carried out in the literature. ${ }^{251,353}$ Starting from the Koopmans picture (KT, see section 4.1.3), i.e. $\Psi_{0}^{N}=\Phi_{0}^{N}=\left|\varphi_{1}, \ldots, \varphi_{N}\right\rangle$ and $\Psi_{\alpha}^{N-1}$ described as single determinants built from a common set

of canonical HF orbitals, only the primary states $\Phi_{k}^{N-1}=a_{k} \Phi_{0}^{N}$ are accessible, with $\chi_{\alpha k}=\delta_{\alpha k}$, and the corresponding ionization potentials (IP)

$$
I P_{k}=E_{k}^{N-1}-E_{0}^{N}=-\varepsilon_{k}
$$

are given by HF orbital energies. Correlation effects affect both IPs and $\chi_{\alpha k}$, but here we are more interested in the amplitudes. The most important correlation effects arise in the ionic states, by the mixing of 2-hole, 1-electron (2h1e) configurations

$$
\Phi_{i j}^{a}=a_{a}^{+} a_{j} a_{i} \Phi_{0}
$$


with the 1 h states $\Phi_{k}$, giving

$$
\Psi_{\alpha}^{N-1}\left(\mathbf{r}_{1}, \ldots, \mathbf{r}_{N-1}\right)=\sum_{k} C_{k, \alpha} \Phi_{k}+\sum_{i<j, a} C_{i j, \alpha}^{a} \Phi_{i j}^{a}
$$

Since intensities are proportional to the square norms of the Dyson orbitals (via square dipole transition amplitudes, eq 47, they depend on $R_{\alpha k}=\chi_{\alpha k}^{2}$, called spectroscopic factors, or pole strengths. Clearly in this case $\chi_{\alpha k}=C_{k, \alpha}$, so that each ionic state receives ("borrows") intensity from the primary states that are mixed into it. This causes the appearance of additional ionic states, so called satellite states, and at the same time the decrease of the intensities of the primary ionic states. Actually, the mixing of such configurations (which become quasi degenerate in the case of inner holes) can be so large as to cause dramatic effects, especially those characterized by a fluctuation of the hole $\Phi_{k} \leftrightarrow \Phi_{i j}^{a}$ (those with $i=k$ or $j=k$ describe relaxation). This mixing can lead to shifts in the values of IPs up to several eV, especially prominent in transition metal compounds, completely subverting the KT ordering of ionic states (breakdown of KT), and the appearance of intense satellite states, to the point that a well defined primary line (e.g. $\left.R_{\alpha k}>0.5\right)$ ceases to exist (breakdown of the one-particle picture $\left.{ }^{251}\right)$.

Based on the ansatz of eq 59 for the initial electronic WP, the time evolution of the ion density matrix $\rho_{i o n}$ and the hole density defined in eq 40 have been considered for a large variety of molecules, and has been recently reviewed. ${ }^{253}$ As has been recognized, realistic pulses will in any case promote ionization out of several orbitals $\varphi_{k}$, and the resulting WP will be more complex. Nonetheless, if a group of final ionic states $\Psi_{\alpha}^{N-1}$ borrow intensity from a single initial orbital $\varphi_{i}$, i.e. $\chi_{\alpha k} \neq 0$ only for $k=i$, their Dyson orbitals $\varphi_{\alpha}^{D}=\chi_{\alpha i} \varphi_{i}$ and the relevant dipole amplitudes will be very similar, so that the SA will correctly describe the relevant WP. Moreover, the full WP could be considered as a linear combination of the individual SA WPs, weighted by the relative dipole amplitudes. In any case it is clear that much can be learnt from the analysis of individual WPs of the SA form eq 59. A universal 
time scale of about 50 as was found for the response of the electron cloud to a sudden ionization, which can be considered a characteristic time of the electron correlation. ${ }^{37}$ Also it was realized the strong connection between correlation effects in the ionization spectrum and the ensuing electron dynamics. Cederbaum and Kuleff ${ }^{253}$ have usefully classified four types of WPs, which are presented in Fig. 24 as a typical bar representation of a molecular photoelectron spectrum, the height of the bars equal to the spectroscopic factors $R_{\alpha k}$, at the corresponding $\mathrm{IP}_{\alpha}$. The color code refers to each initial orbital $\varphi_{k}$.

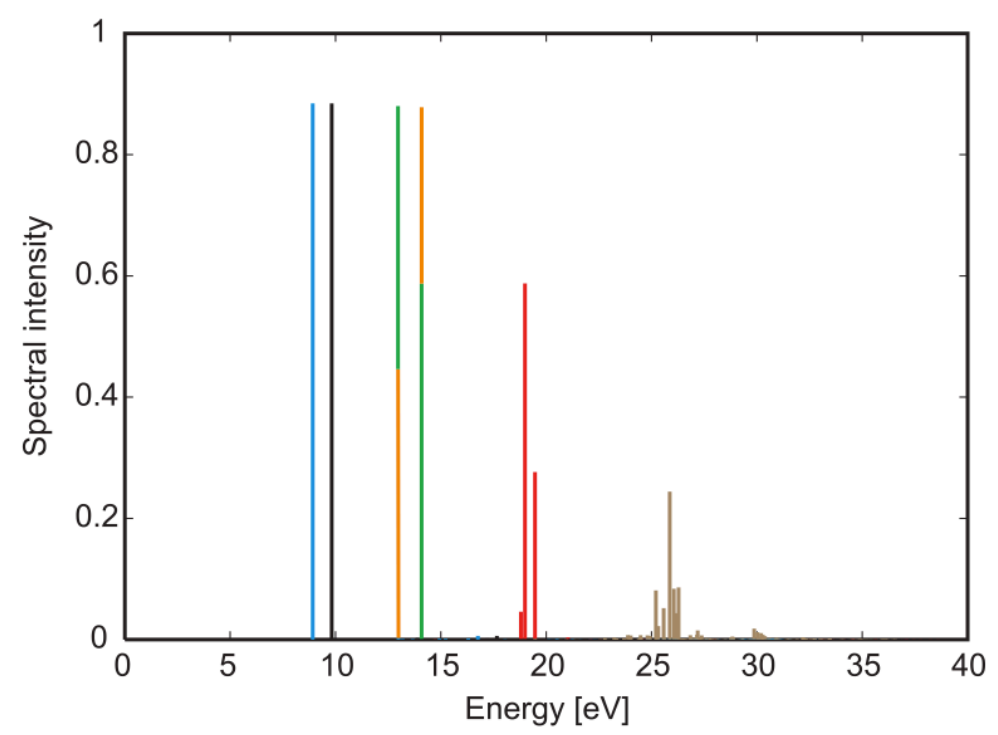

Figure 24: Typical ionization spectrum of a molecule. Each vertical line is related to a cationic eigenstate and is located at the corresponding ionization energy. The height of the line represents the spectroscopic factor $R_{\alpha k}$. The different colours in the lines correspond to the contributions of different $1 \mathrm{~h}$ configurations, describing an electron missing from a particular orbital. From ref. 253 with permission. Copyright 2014 IOP Publishing.

The simplest (less interesting) situation is when KT is well verified, as it is often the case for ionizations of the outermost orbitals. In this case the primary state takes almost all of the intensity, $R_{\alpha k} \simeq 1$ for $\alpha=k$, and there are no appreciable satellites. These correspond to the first two lines in Fig. 24. Since the WP is essentially composed of a single hole state, it remains almost stationary and no significant charge migration takes place. As we will see in the following sections, this is not the case when the WP is composed of several single-hole states, as for ionization induced by an attosecond pulse. The second situation is 
rather curious, and it is called hole mixing. In the simplest case it involves two ionic states, $\Psi_{\alpha_{1}}^{N-1}$ and $\Psi_{\alpha_{2}}^{N-1}$, with Dyson orbital square norm, i.e. total spectroscopic strength, close to one, but with comparable $R_{\alpha k}$ from two initial orbitals $\varphi_{k_{1}}, \varphi_{k_{2}}$. This is not an uncommon situation, although it is entirely due to correlation (relaxation) in the KT picture. It is exemplified by lines three and four in Fig. 24, with green and orange colors showing the two orbital contributions. It is a situation where the SA ansatz is unclear, as the two ionic states can still be considered as two individual primary states at the Dyson orbital level, and the resulting WP should be better evaluated from the corresponding dipole amplitudes. Keeping the SA ansatz, each WP

$$
a_{k_{i}} \Psi_{0}^{N}=c_{1 i} \Psi_{\alpha_{1}}^{N-1}+c_{2 i} \Psi_{\alpha_{2}}^{N-1}
$$

is a superposition of the two cationic states, and will oscillate with the bohr frequency $\hbar \omega=\left|E_{\alpha_{2}}-E_{\alpha_{1}}\right|$. It amounts to the hole oscillating between orbitals $\varphi_{k_{1}}$ and $\varphi_{k_{2}}$. The third situation occurs when ionization out of orbital $\varphi_{k_{1}}$ gives rise to a main line accompanied by a strong satellite (the red lines in Fig. 24), generally associated with dominant 2h1e configurations $\Phi_{i j}^{a}$. Again this will generate a wave packet superposition of the main ionic state and the satellite. This can be further analyzed depending on the nature of the satellite. If $i, j \neq k$, it is a pure correlation satellite, and oscillation will entail shifting of the hole from $k$ to $i$, plus simultaneous excitation $j \rightarrow a$. If $k=i$, one has a relaxation satellite (the type that can be described at the $\triangle \mathrm{SCF}$ level). The hole stays fixed, but one can still have charge migration due to the $j \rightarrow a$ excitation. The latter will often correspond to a charge transfer excitation, which screens the original hole ( $a$ localized close to $i$ ), and it is expected to be particularly prominent when strong relaxation effects affect the hole formation, an extreme example being the appearance of giant satellites upon core ionization of chemisorbed species, like $\mathrm{CO}$ or $\mathrm{N}_{2}$ on $\mathrm{Ni}{ }^{354,355}$ Finally, ionization of inner valence orbitals often produces the complete breakdown of the one particle picture, as exemplified by the brown lines in Fig. 24. 
In this case, the WP will be a superposition of many states, which form a quasicontinuum if they lie below the double ionization threshold, or a real continuum if they lie above, and the wave packet $a_{k} \Psi_{0}^{N}$ is a resonance in such continuum. In that case it will decay almost exponentially, either delocalizing the charge over a multitude of orbitals, and over the whole molecular skeleton, or promote a second ionization. Examples of all situations have been described in a number of studies. ${ }^{34,338,339,356-358}$ In many cases, the positive charge initially localized on a specific functional group of the molecule can migrate to another, even spatially well separated, in a few femtoseconds. This is due to the superposition of several $1 \mathrm{~h}$ main states, generally mediated by the $2 \mathrm{~h} 1 \mathrm{e}$ configurations, and as such do not necessarily correspond to a flow of charge along the skeleton, the hole disappearing from one end and

appearing on the other. Such is, for instance, the case in the Gly-Gly-NH-CH 3 molecule $^{-}$ studied in Ref. 338, or the polypeptide considered in Ref. 35.

\subsubsection{Methods explicitly including the ionization step}

As explained in the previous chapter, theoretical methods able to provide ionization amplitudes for large molecules make use of a single-determinant approach for both the ground state of the neutral molecule and all final continuum states resulting from the interaction with an attosecond pulse. One of these methods is the static exchange DFT method described in section 4.1.7, which provides the necessary dipole coupling matrix elements to evaluate the amplitudes (39) for all open ionization channels. In this DFT-like formalism, but also within a HF framework, the electron density (42) for a given target in its ground state, $\rho_{\text {neutral }}$, can be directly written as the sum of the squares of the individual Kohn-Sham spinorbitals $\varphi_{\alpha}(\mathbf{r})$ that define the ground state $\Psi_{0}^{N}\left(\mathbf{r}_{1}, \mathbf{r}_{2}, \ldots, \mathbf{r}_{N}\right)$ :

$$
\rho_{\text {neutral }}(\mathbf{r})=\sum_{\alpha=1}^{N} \varphi_{\alpha}^{2}(\mathbf{r})
$$


The electron density of the ion, $\rho_{\text {ion }}(\mathbf{r}, t)$, which allows one to track the charge evolution after molecular ionization, can be defined either from an $N$-electron target, including the escaping electron, or from an $(N-1)$-electron target, assuming that the photoelectron and the remaining ion are readily decoupled.

As discussed in section 4.1 .3 (see eq. 45), the $N$-electron continuum states that describe the ionized system in the static exchange DFT formalism, assuming frozen nuclei, are written as the antisymmetrized product of the cationic wave function $\Psi_{\alpha}^{N-1}\left(\mathbf{r}_{1}, \ldots, \mathbf{r}_{N-1}\right)$, represented by the single Slater determinant obtained after removal of the $\phi_{\alpha}$ Kohn-Sham spinorbital from the ground state of the $N$-electron system, and a one-electron continuum function $\varphi_{\alpha l \varepsilon}\left(\mathbf{r}_{N}\right)$ representing a photoelectron ejected with energy $\epsilon$ and angular momentum $l$ that leaves behind an $\alpha$ ionic state (for the sake of clarity, we have dropped the index $m$ in eq 45 and used a common label $\varepsilon$ to indicate the energy of the continuum electron in all ionization channels). The total energy of the final state, $E_{n}$ in 35 and 39 is given by $E_{n}=E_{\alpha}+\varepsilon$, the sum of the photoelectron energy $\varepsilon$ and the energy of the electronic state of the cation $E_{\alpha}$. In this single-determinant approach, the $N$-electron density is given by: ${ }^{359}$

$$
\begin{aligned}
& \rho_{\text {ion }}\left(\mathbf{r}_{1}, t\right)=\sum_{\alpha, l} \int \mathrm{d} \varepsilon\left[\left(\sum_{\alpha^{\prime} \neq \alpha}\left|\varphi_{\alpha^{\prime}}\left(\mathbf{r}_{1}\right)\right|^{2}+\left|\varphi_{\alpha l \varepsilon}\left(\mathbf{r}_{1}\right)\right|^{2}\right)\left|c_{\alpha l \varepsilon}\right|^{2}\right. \\
+ & \sum_{\alpha^{\prime} \neq \alpha} c_{\alpha l \varepsilon}^{*} c_{\alpha^{\prime} l \varepsilon} \varphi_{\alpha}\left(\mathbf{r}_{1}\right) \varphi_{\alpha^{\prime}}\left(\mathbf{r}_{1}\right) e^{-i\left(E_{\alpha^{\prime}}-E_{\alpha}\right) t} \\
+ & \left.\sum_{l^{\prime}} \int \mathrm{d} \varepsilon^{\prime} c_{\alpha l \varepsilon}^{*} c_{\alpha l^{\prime} \varepsilon^{\prime}} \varphi_{\alpha l \varepsilon}\left(\mathbf{r}_{1}\right) \varphi_{\alpha l^{\prime} \varepsilon}\left(\mathbf{r}_{1}\right) e^{-i\left(\varepsilon^{\prime}-\varepsilon\right) t}\right]
\end{aligned}
$$

where $\varphi_{\alpha}$ are the Kohn-Sham orbitals and $c_{\alpha l \varepsilon}$ are the ionization amplitudes obtained from eq 39.

Alternatively one can define a reduced $(N-1)$-electron density matrix relative to the cation by taking the trace over the photoelectron degrees of freedom

$$
\rho_{i o n}^{N-1}\left(\mathbf{r}_{1}, \mathbf{r}_{2}, \ldots, \mathbf{r}_{N-1}, t\right)=\sum_{\alpha, l} \int \mathrm{d} \varepsilon\left\langle\varphi_{\alpha l \varepsilon} e^{-i \varepsilon t} \mid \Psi_{N}\right\rangle\left\langle\Psi_{N} \mid \varphi_{\alpha l \varepsilon} e^{-i \varepsilon t}\right\rangle
$$


where the bracket notation is used for the integral over the spatial coordinate of the $N$ th electron. We can then use the one-particle electron density definition as in eq 42 and integrate over the coordinates of $(N-2)$ electrons to obtain a more simplified version of the electron density of the ion:

$$
\rho_{i o n}^{N-1}(\mathbf{r}, t)=\sum_{\alpha} \varphi_{\alpha}^{2}(\mathbf{r}) \sum_{\alpha^{\prime} \neq \alpha} \gamma_{\alpha^{\prime} \alpha^{\prime}}^{(i o n)}-\sum_{\alpha} \sum_{\alpha \neq \alpha^{\prime}} \gamma_{\alpha \alpha^{\prime}}^{(i o n)} e^{-i\left(E_{\alpha}-E_{\alpha^{\prime}}\right) t} \varphi_{\alpha}(\mathbf{r}) \varphi_{\alpha^{\prime}}(\mathbf{r})
$$

where $\gamma_{\alpha \alpha^{\prime}}^{(i o n)}$ is the reduced density matrix:

$$
\gamma_{\alpha \alpha^{\prime}}^{(i o n)}=\sum_{l} \int c_{\alpha \varepsilon l}(T) c_{\alpha^{\prime} \varepsilon l}^{*}(T) d \varepsilon
$$

Note that eq 63 and eq 64 lead to the same result as soon as the ionized electron is far away from the ion. The decoupling between the photoelectron and the remaining cation will occur at different times depending on the cationic state and on the photoelectron energy, but even in the less favorable scenario, it is estimated to occur in less than one femtosecond, ${ }^{359}$ thus validating the widely employed $(N-1)$-electron density expression.

The above formalism has been successfully employed to describe charge migration induced by attosecond pulses in the phenylalanine ${ }^{25,254}$ and glycine ${ }^{360}$ cations.

\subsection{Nuclear degrees of freedom}

Pure $a b$ initio description of the photoionization process in polyatomic molecules, including all electronic and nuclear degrees of freedom, will still remain a challenge, at least for a few years. Theoretical treatments using a fully dimensional quantum representation of electronic and nuclear motions on an equal footing, beyond the Born-Oppenheimer approximation, is only available for the smallest diatomic molecular targets $\left(\mathrm{H}_{2}^{+}\right.$and $\mathrm{H}_{2}$ and their isotopes). ${ }^{32}$ The Born-Oppenheimer approximation, separating electronic and nuclear degrees of freedom, is the first and most common approach to treat molecular targets, with the field-free 
hamiltonian being written as:

$$
\mathcal{H}_{0}=\mathcal{H}_{e l}+T_{\mathbf{R}}
$$

where

$$
\mathcal{H}_{e l}=-\sum_{i=1}^{N} \frac{1}{2 m_{e}} \nabla_{i}^{2}+\sum_{i=1}^{N} \sum_{i>j} \frac{1}{\left|\mathbf{r}_{i}-\mathbf{r}_{j}\right|}-\sum_{i=1}^{N} \sum_{I=1}^{M} \frac{Z_{I}}{\left|\mathbf{r}_{i}-\mathbf{R}_{I}\right|}+\sum_{I=1}^{M} \sum_{J>I} \frac{Z_{I} Z_{J}}{\left|\mathbf{R}_{I}-\mathbf{R}_{J}\right|}
$$

and

$$
T_{\mathbf{R}}=-\sum_{I=1}^{M} \frac{1}{2 M_{I}} \nabla_{\mathbf{R}_{I}}^{2}
$$

where the mass polarization terms and relativistic effects are neglected. The total wave function, $\Psi(\mathbf{r}, \mathbf{R})$ can then be written as a product of an electronic $\psi_{n}\left(\mathbf{r}_{1}, \ldots, \mathbf{r}_{N} ; \mathbf{R}_{1}, \ldots, \mathbf{R}_{M}\right)$ and an associated nuclear wave function $\chi_{n, v_{n}}\left(\mathbf{R}_{1}, \ldots, \mathbf{R}_{M}\right)$. One can then separately solve the time-independent Schrödinger equations for the electronic,

$$
\mathcal{H}_{e l} \psi_{n}\left(\mathbf{r}_{1}, \ldots, \mathbf{r}_{N} ; \mathbf{R}_{1}, \ldots, \mathbf{R}_{M}\right)=\varepsilon_{n}\left(\mathbf{R}_{1}, \ldots, \mathbf{R}_{M}\right) \psi_{n}\left(\mathbf{r}_{1}, \ldots, \mathbf{r}_{N} ; \mathbf{R}_{1}, \ldots, \mathbf{R}_{M}\right)
$$

and nuclear components,

$$
\left[T_{\mathbf{R}}-\varepsilon_{n}\left(\mathbf{R}_{1}, \ldots, \mathbf{R}_{M}\right)\right] \chi_{n, v_{n}}\left(\mathbf{R}_{1}, \ldots, \mathbf{R}_{M}\right)=E_{n, v_{n}} \chi_{n, v_{n}}\left(\mathbf{R}_{1}, \ldots, \mathbf{R}_{M}\right)
$$

This approximation is valid as long as conical intersections or avoided crossings do not play an important role. Despite the numerical and computational advantages of separately solving eq 69 and eq 70, the solution of the second one requires an accurate evaluation of the potential energy surfaces, i.e. solving eq 69 for all possible nuclear geometries. It implies a fine numerical scan over the $3 M-6$ vibrational modes. Moreover, to describe light-matter interactions, calculation of the dipole transition elements is required. This obviously becomes an impossible task as the number of atomic centers increases. However, in several cases, it is still possible to achieve good accuracy by reducing the number of vibrational modes involved. This is the case, e.g., of recent theoretical and experimental works on vibrationally resolved 
photoelectron spectra of polyatomic molecules at high photon energies, such as those for $\mathrm{BF}_{3},{ }^{361} \mathrm{CH}_{4},{ }^{362} \mathrm{CF}_{4},{ }^{363} \mathrm{SF}_{6} \cdot{ }^{364}$ In these experiments, the measured spectra suggest a major contribution of the symmetric stretching mode, thus reducing the eigenvalue problem defined in 70 to one nuclear degree of freedom (the internuclear distance between the central and the surrounding atoms). Therefore, one only needs to evaluate a set of potential energy curves, $E_{n}(R)$, from eq 69 , instead of $3 M-6$ dimensional surfaces. The dipole matrix elements for the transition from the ground state to a given final state are directly computed as:

$$
\left\langle\Psi_{E_{f}}(\mathbf{r}, R)\left|\sum_{k} \mathbf{d}_{k}\right| \Psi_{0}(\mathbf{r}, R)\right\rangle=\int d R \chi_{n, v_{n}}(R)\left\langle\psi_{n}(\mathbf{r}, R)\left|\sum_{k} \mathbf{d}_{k}\right| \psi_{0}(\mathbf{r}, R)\right\rangle_{\mathbf{r}} \chi_{0, v=0}(R)
$$

where $\sum_{k} \mathbf{d}_{k}$ is the multi-electronic dipole operator and the brackets imply the integral over the electronic coordinates. Obviously, the above expressions are only useful for relatively small polyatomic molecules with high symmetry and for specific problems where the dimensionality, as we have seen, can be reduced. Otherwise, the common alternative is to obtain a quantum description of electrons while using classical mechanics for nuclear motion. This approach has been widely used for the investigation of molecular dynamics associated with excited states of neutral molecules, ${ }^{365-367}$ where the available standard quantum chemistry packages are combined with Ehrenfest molecular dynamics ${ }^{268,368-370}$ or with the more elaborate surface-hopping approaches. ${ }^{371-375}$ More recently, these schemes are now being extended to photoionization studies to account for the nuclear degrees of freedom, ${ }^{359}$ where the main limitation lies on achieving a reliable description of the electronic continuum structure as discussed in the previous section.

There is an increasing interest in investigating the role of nuclear motion in charge migration processes in biomolecules. The charge redistribution over the molecular skeleton of relatively large molecules has been theoretically predicted to occur in a few femtoseconds ${ }^{34,35,267,358}$ and recently experimentally proved in an amino acid. ${ }^{25}$ In such short time scales, one would expect nuclear motion to play a minor role, since nuclear motion takes place 
in tens of femtoseconds. Nevertheless, it is relevant to understand how the electronic and nuclear degrees of freedom are coupled and how this coupling affects the charge migration process. Ultimately, it is to be explored to which extent the ultrafast electronic dynamics can be interrupted or guided to localize the charge in an specific site and drive the fragmentation or reaction path into the desired channel. Very recent theoretical efforts are being devoted to address this issues. For instance, in ${ }^{376}$ the hole migration process has been simulated and averaged for several distorted geometries of the benzene molecule. The results suggest that electron coherences should remain for at least $\approx 10$ fs. The electron dynamics was simulated using quantum chemistry packages based on a multi-configuration interaction picture that accounts for electron correlation effects. In a more elaborate approach applied to molecular targets of similar structure, ${ }^{252,268}$ the nuclear motion was included by using Ehrenfest dynamics, ${ }^{374,377}$ mixing quantum and classical dynamics methods. The nuclear wave packet evolution was obtained by integrating the classical Newton's equations of motion, following the gradient given by a mixture of two cationic electronic states. The electronic wave function was thus computed on the fly, every time step, in a state-averaged complete active space self-consistent field (CASSCF) level, using standard quantum chemistry packages. The electronic and the classical nuclear degrees of freedom were connected in a mean-field manner. In these methods, the non-adiabatic effects are reasonably described and are in fact responsible for a fast decoherence observed in the electronic dynamics. Although charge migration remains observable in the first few femtoseconds, it is rapidly washed out (in less than 10 fs) due to the nuclear rearrangement. It is worth noticing that these works were performed in the particular situation in which the nuclear wave packet passes through conical intersections (avoided crossings) in the electronic potential energy surfaces (curves) of the cation, where non-adiabatic couplings play a crucial role and a high degree of decoherence is expected to be introduced in the electronic dynamics. For instance, ultrafast electronic coherence will be preserved for longer times when the laser-induced molecular wave packet is associated with electronic cationic states whose potential energy surfaces (curves) run parallel. Similar 
strategies are nowadays being employed for larger molecules, in which the electron dynamics after photoionization is described in a quantum manner by using TDDFT-based methods and the associated nuclear motion is described with classical methods. ${ }^{368-370}$ In this context, non adiabatic effects are also explicitly included. The latter method has been used to investigate the evolution of valence-shell vacancies in the glycine amino acid. ${ }^{359}$ The results of this work have shown that the first charge fluctuations between different molecular centers

remain unaltered by the nuclear motion for $\approx 10 \mathrm{fs}$, in agreement with the findings of. ${ }^{376}$ At later times, charge fluctuations are significantly affected by the nuclear dynamics, but they are still observable at least during the following 10-15 fs. For further discussion see Sect. 6.2 .

\subsection{HHG methods}

In the last few years HHG has emerged as an alternative way of looking at charge migration processes in molecules. As pointed out in Sect. 3.2.2, the idea is that the dynamics induced by a strong laser pulse leaves its signature in the emitted light through the electron recombination process that can occur only half a cycle after such dynamics was initiated. Therefore, the HHG process itself provides the necessary sub femtosecond time resolution required to resolve electron wave packet dynamics, without the need of a probe laser pulse. So far, the technique has been applied to study charge migration in small polyatomic molecules, but it promises to shed additional light on more complex systems.

For effective one- and two-electron diatomic molecules, the high harmonic spectrum can be directly obtained from the solutions of the time-dependent Schrödinger equation (TDSE). In the case of a one-electron diatomic molecule, ${ }^{378-380}$ this equation can be solved almost exactly if one neglects rotational effects, which is justified because rotation is much slower than electronic and vibrational motions and the IR pulses used to induce the HHG process 
are very short. The corresponding equation reads, in full dimensionality,

$$
\left[H_{e l}(\mathbf{r}, R)+T(R)+V(\mathbf{r}, t)-i \frac{\partial}{\partial t}\right] \Psi(\mathbf{r}, R, t)=0
$$

where $R$ is the internuclear distance, $\mathbf{r}$ denotes the position of the electron, $H_{e l}=T_{e l}+V_{e N}+$ $1 / R$ is the electronic time-independent hamiltonian, $T(R)$ the nuclear kinetic energy operator and $V(\mathbf{r}, t)$ the external potential created by the laser field. Likewise, $T_{e l}$ and $V_{e N}$ stand for the electron kinetic energy operator and the electron-nuclei interaction, respectively. The TDSE can be numerically solved by using a sufficiently dense spatial grid and by placing absorbers at sufficiently long distances as to avoid spurious reflections. Time propagation is performed by using different numerical procedures. At each time step, one has to compute the dipole response, $\ddot{d}(t)=-\langle\Psi(\mathbf{r}, R, t)|\mathbf{d}(\mathbf{r}, t)| \Psi(\mathbf{r}, R, t)\rangle$, where $\mathbf{d}$ is the dipole operator, which is usually written in the acceleration form. The harmonic spectrum is given by the square of the Fourier transform of $\ddot{d}(t)$.

For more complex molecules, solution of the TDSE in full dimensionality is no longer possible due to the large number of electronic and nuclear degrees of freedom. Thus, most theoretical calculations rely on extensions of the semiclassical three-step model described in Sect. 2.1, which was initially developed for effective one-electron systems. However, in an $N$ electron system, there are several ways of sharing the energy between the ejected electron and the remaining $(N-1)$-electron molecular cation, each of those corresponding to a different ionization channel. Therefore, an ejected electron that leaves behind a molecular cation in a particular state may not necessarily recombine with the cation in that same state. Indeed, during the round trip of the electron between the ionization and the recombinations steps, the laser can also induce transitions between the electronic states of the cation thus leading to a coherent superposition of cationic states, hence to charge migration processes similar to those discussed in 4.2.2. Upon recombination of the ionized electron with the cation, the signature of such dynamics will be imprinted in the HHG spectrum. To account for this 
complex dynamics, one has to generalize eq 18 and include all accessible electronic states of the cation. For this, we will closely follow the formalism as described by Smirnova and Ivanov. ${ }^{52}$ In this case, the total dipole response of the system will be given by the coherent superposition of the dipoles $\left\langle\mathbf{r}_{\alpha \beta}>\right.$ associated with each ionization-recombination channel:

$$
<\mathbf{r}(t)>=\sum_{\beta, \alpha}<\mathbf{r}^{(\beta \alpha)}(t)>
$$

Here $\left\langle\mathbf{r}_{\beta \alpha}>\right.$ is the multi-electron dipole associated with leaving the cation in the $\alpha$ state and recombination with the cation in the $\beta$ state. Assuming that the dipole operator only acts on the ionized electron, not on the cation, the $\left\langle\mathbf{r}_{\beta \alpha}\right\rangle$ dipole takes on the form:

$$
<\mathbf{r}^{(\beta \alpha)}(t)>=i \int_{0}^{t} d t^{\prime} \int d^{3} \mathbf{p} \mathbf{d}_{\beta}^{*}(\mathbf{p}+\mathbf{A}(t)) a_{\beta \alpha}\left(t, t^{\prime}\right) e^{-i S\left(\mathbf{p}, t, t^{\prime}\right)} \mathbf{E}\left(t^{\prime}\right) \mathbf{d}_{\alpha}\left(\mathbf{p}+\mathbf{A}\left(t^{\prime}\right)\right)
$$

where $a_{\beta \alpha}\left(t, t^{\prime}\right)$ is the time-dependent transition amplitude between the state $\alpha$ populated at time $t^{\prime}$ (the ionization time) and the state $\beta$ at time $t$,

$$
\begin{gathered}
\mathbf{d}_{\alpha}(\mathbf{p}+\mathbf{A}(t))=\left\langle\mathbf{p}+\mathbf{A}(t)|\mathbf{d}| \varphi_{\alpha}^{D}\right\rangle, \\
S\left(\mathbf{p}, t, t^{\prime}\right)=\frac{1}{2} \int_{t^{\prime}}^{t}[\mathbf{p}+\mathbf{A}(\tau)]^{2} d \tau+I_{p}\left(t-t^{\prime}\right),
\end{gathered}
$$

$\varphi_{\alpha}^{D}$ is the channel specific Dyson orbital defined as in eq 46, which represents the overlap between the $N$-electron ground state wave function of the neutral molecule and the $(N-1)$ electron wave function of the $\alpha$ state of the molecular cation, and all the other notations are as defined in 4.1. As can be seen, eq 74 is very similar to eq 18 for the one-electron dipole, except for the fact that one has to coherently sum the dipoles associated with the different ionization-recombination channels and the initial ground state has been replaced by the corresponding Dyson orbitals. The amplitudes $a_{\beta \alpha}\left(t, t^{\prime}\right)$ are usually obtained by solving 
the TDSE in the subspace of all cationic states efficiently coupled by the strong laser field.

Applying the saddle point method for the variables $t, t^{\prime}$ and $\mathbf{p}$, as described in Sect. 2.3, leads to the half-cycle dipole for every given ionization - recombination channel:

$$
<\mathbf{r}^{(j, \beta \alpha)}(t)>=a_{r e c}^{\beta}\left(\mathbf{p}_{s}, t\right) a_{\text {prop }}^{\beta \alpha}\left(\mathbf{p}_{s}, t, t_{i}\right) a_{\text {ion }}^{\alpha}\left(\mathbf{p}_{s}, t_{i}\right),
$$

where

$$
\begin{aligned}
& a_{\text {ion }}^{\alpha}\left(\mathbf{p}_{s}, t_{i}\right)=\left[\frac{2 \pi}{S_{t_{i}, t_{i}}^{\prime \prime}}\right]^{1 / 2} e^{-i S\left(\mathbf{p}_{s}, t_{i}^{\prime}, t_{i}\right)} \mathbf{E}\left(t_{i}\right) \mathbf{d}_{\alpha}\left(\mathbf{p}_{s}+\mathbf{A}\left(t_{i}\right)\right), \\
& a_{\text {prop }}^{\beta \alpha}\left(\mathbf{p}_{s}, t, t_{i}\right)=\frac{(2 \pi)^{3 / 2}}{\left(t-t_{i}\right)^{3 / 2}} e^{-i S\left(\mathbf{p}_{s}, t, t_{i}^{\prime}\right)} a_{\beta \alpha}\left(t, t_{i}\right), \\
& a_{r e c}^{\beta}=\mathbf{d}_{\beta}^{*}\left(\mathbf{p}_{s}+\mathbf{A}(t)\right) .
\end{aligned}
$$

and $S_{t_{i}, t_{i}}^{\prime \prime}$ is the second derivative of the action calculated at time $t_{i}$. Notice that the propagation amplitude includes the laser - induced dynamics in the ion $a_{\beta \alpha}(t)$. The full dipole for each ionization-recombination channel is the sum over the different half-cycles and the harmonic spectrum results from the FFT of the full dipole $\mathbf{r}_{\beta \alpha}(N \omega)$ :

$$
\begin{aligned}
& <\mathbf{r}^{(\beta \alpha)}(t)>=\sum_{j}<\mathbf{r}^{(j, \beta \alpha)}(t)> \\
& \mathbf{r}^{(\beta \alpha)}(N \omega)=\int d t<\mathbf{r}^{(\beta \alpha)}(t)>e^{i N \omega t} .
\end{aligned}
$$

The complete harmonic response is obtained by adding coherently the contributions of all ionization - recombination channels.

In the last few years this formalism has been extended to include the effect of nuclear dynamics. This effect is usually included through the formula ${ }^{188}$

$$
<\mathbf{r}^{(j, \beta \alpha)}(t)>=a_{r e c}^{\beta}\left(\mathbf{p}_{s}, t\right) a_{\text {prop }}^{\beta \alpha}\left(\mathbf{p}_{s}, t, t_{i}\right) C\left(t-t_{i}\right) a_{i o n}^{\alpha}\left(\mathbf{p}_{s}, t_{i}\right)
$$

where $C\left(t-t_{i}\right)$ is the nuclear autocorrelation function, ${ }^{381}$ which is the overlap integral of the 
cationic nuclear wave packet launched at the moment of ionization, $t_{i}$, and the corresponding time-evolved wave packet at time $t$. Notice that the nuclear autocorrelation function appears as a global factor because in deriving the above equation we have assumed that the dipoles do not depend on the position of the nuclei, which is not always the case, especially when the nuclei have had enough time to move outside the Franck-Condon region before the round trip of the electron is initiated. Going beyond this approximation implies that integrals cannot be factorized as products of electronic and nuclear integrals, and that the dipoles given by eq 75 must include the nuclear degrees of freedom from the very beginning.

Also, one can go beyond the Dyson-orbital picture to fully account for the dipole coupling between the $N$-electron wave functions by replacing eq 75 by the more precise one

$$
\mathbf{d}_{\alpha}(\mathbf{p}+\mathbf{A}(t))=\left\langle\Psi_{\alpha}^{N-1} \times[\mathbf{p}+\mathbf{A}(t)]\left|\sum_{k}^{N} \mathbf{d}_{k}\right| \Psi_{g}^{N}\right\rangle
$$

which includes all possible dipole transitions involving the continuum electron.

In order to apply the above formalism, one has to evaluate the $N$ and $N-1$ electronic wave functions in a grid of molecular geometries by using standard quantum chemistry methods, the nuclear wave packet that propagates on the potential energy surfaces of the cation, and the TDSE than describes the laser-induced dynamics in the cation. More recent attempts go beyond the strong-field approximation and make use of recombination dipoles $\mathbf{d}_{\alpha}(\mathbf{p}+\mathbf{A}(t))$ calculated from ab initio approaches.

\section{$5 \quad$ From diatomic to polyatomic molecules}

One of the most successful applications of attosecond technology has been the development of pump-probe schemes to visualize electron molecular dynamics in real time. Following the footsteps of femtochemistry, which allows one to guide nuclear rearrangements in molecules, the ultimate goal of attosecond technology is to achieve a similar control of electronic processes at the sub-femtosecond time scale. These ultrafast phenomena were first investigated 
in the smallest molecules, for which reliable theoretical simulations were possible, and are progressively considering larger molecules. The present section is devoted to shortly review some of the most remarkable experimental and theoretical achievements using attosecond pump-probe schemes in relatively small molecules, going from diatomics $\left(\mathrm{H}_{2}, \mathrm{D}_{2}, \mathrm{~N}_{2}\right.$, etc) to small polyatomic targets $\left(\mathrm{CO}_{2}, \mathrm{C}_{2} \mathrm{H}_{4}\right.$, etc).

\subsection{Attosecond pump-probe techniques applied to hydrogen molecules}

A peculiarity of hydrogen molecules is that their nuclei are light, so they can move relatively fast $^{382,383}$ and, in processes such as dissociative autoionization, they can do so in a time scale comparable to that of electronic motion. ${ }^{27,28}$ Therefore, they are ideal systems to investigate coupled electron and nuclear dynamics with high accuracy. In 2004 Bandrauk et al. studied the process of electron localization on $\mathrm{H}_{2}^{+}$molecular ions by using TDSE applied to a one-dimensional model non-Born-Oppenheimer $\mathrm{H}_{2}^{+}$molecule. ${ }^{384}$ Charge oscillations with a period of 350 as were calculated, as a function of the delay between a 0.8 -fs, 115 -nm pump pulse and a $0.1-\mathrm{fs}, 20-\mathrm{nm}$ probe pulse. A very remarkable finding resulting from ultrafast experiments performed with hydrogen molecules was the demonstration and control of electron localization following dissociative ionization. Pioneering work published in 2006, which made use of IR fields only, ${ }^{385}$ succeeded in achieving control of electron localization by modifying the relative CEP. As described in Sect. 3.2, only a few years later, trains of attosecond pulses ${ }^{386}$ or even isolated attosecond pulses ${ }^{33}$ were combined with IR fields to manipulate electron localization by varying the pump-probe time delay. In the following, we describe in some detail the latter work since it was the first ever application of attosecond EUV-pump / IR-probe schemes to molecules, namely $\mathrm{D}_{2}$, by using single attosecond pump pulses. This is essentially the scheme that has been subsequently used in experiments performed in more complex molecular systems, which will be described later in this review. 


\subsubsection{EUV-pump/IR-probe}

In spite of the technical difficulties to generate single attosecond pulses, the use of these pulses has several advantages with respect to using trains of attosecond pulses pulses. ${ }^{32}$ Indeed, a train of pulses launches replicas of electronic wave packets that interfere with each other, while isolated pulses trigger the dynamics of a single electronic wave packet that can thus evolve freely before the probe pulse arrives. The dynamics induced in the second case is thus simpler to analyze. In the work of Sansone et al, ${ }^{33}$ localization of the electronic charge distribution was controlled by varying the time delay between an isolated 300-400 as pulse and a few-cycle 6 fs IR pulse. The attosecond pulse, which covered a spectral range between 20 and $40 \mathrm{eV}$, was produced from HHG in a krypton gas jet irradiated with linearly polarized few-cycle IR pulses. ${ }^{33,133}$ The three-dimensional velocity distribution of the $\mathrm{D}^{+}$ions was retrieved by using a velocity-map imaging spectrometer as that described in section 3.2.1. The experimental observable was the asymmetry that appears in the angle-differential momentum distributions of the charged $\mathrm{D}^{+}$fragments upon dissociative ionization. The well-known dipole selection rules imply that, for molecules oriented parallel to polarization direction of the EUV pulse, one-photon absorption from the $\mathrm{X}^{1} \Sigma_{g}^{+}$ground state of the molecule can only reach states of ${ }^{1} \Sigma_{u}^{+}$symmetry. Nevertheless, because in the experimental set up the protons were collected in a $45^{\circ}$ cone around the polarization axis, some contribution from ${ }^{1} \Pi_{u}$ states could not be discarded. Under these conditions, the absorption of photons between 20-40 $\mathrm{eV}$ leads to direct ionization that can leave the molecular ion in the $1 \mathrm{~s} \sigma_{g}$ and $2 \mathrm{p} \sigma_{u}$ states, which respectively lead to different proton kinetic energies (an illustration of the potential energy curves is given in right-hand side panels of Fig. 25). Indeed, while the Franck-Condon overlap between the ground state of $\mathrm{D}_{2}$ and the $1 \mathrm{~s} \sigma_{g}$ state of $\mathrm{D}_{2}^{+}$favors low nuclear kinetic energies $(<1 \mathrm{eV})$, the overlap between the former state and the first excited state of the ion, $2 \mathrm{p} \sigma_{u}$, favors nuclear kinetic energies in the interval 5-10 eV. Furthermore, due to the large bandwidth of the attosecond pulse, doubly excited states embedded in the electronic continua can also be populated. These are autoionizing states that can lead to fast molecu- 

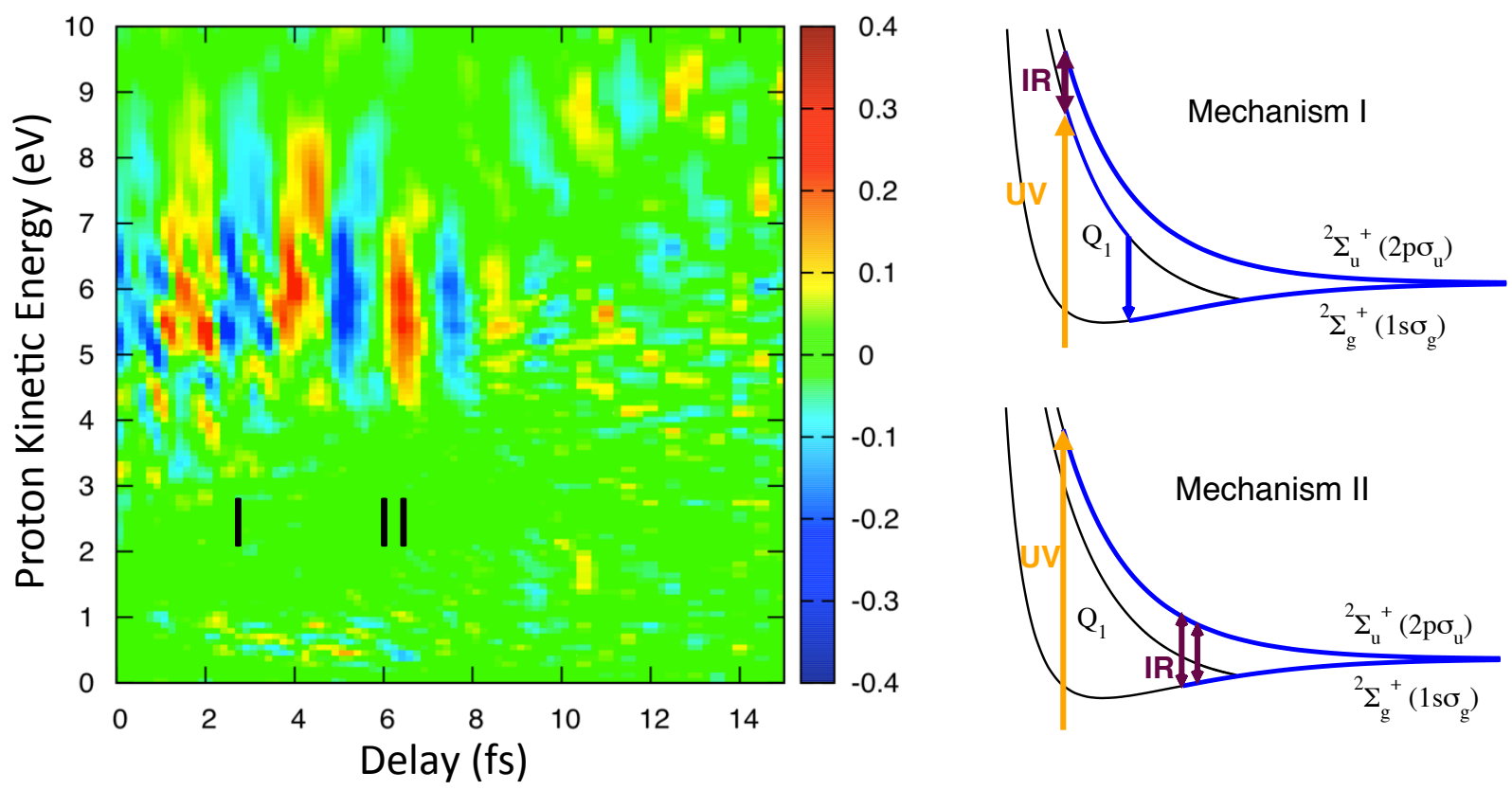

Figure 25: Calculated asymmetry parameter for the formation of protons as a function of the ion kinetic energy ( $\mathrm{y}$-axis) and the pump-probe delay (x-axis). The right panels show the relevant potential energy curves and illustrate the two mechanisms, I and II, responsible for the observed asymmetry. Orange arrows indicate one-photon transitions induced by the EUV pump pulse; purple double arrows indicate one-photon absorption or emission induced by the IR probe pulse; thick blue lines show the different classical paths that the nuclei can follow as a result of the interaction with the pump and probe pulses.

lar dissociation while the electron is still being ejected to the continuum, thus leading to a characteristic range of nuclear kinetic energies. As demonstrated in early works, ${ }^{27,28,301,387}$ because of their large oscillator strength, the absorption of photons in the range between 20 and $40 \mathrm{eV}$ is more likely to populate the lowest autoionizing state of the $\mathrm{Q}_{1}{ }^{1} \Sigma_{u}^{+}$series that converges into the $2 \mathrm{p} \sigma_{u}$ ionization threshold. When contribution from molecules oriented perpendicularly to the laser polarization direction cannot be excluded, also the lowest state of the $\mathrm{Q}_{2}{ }^{1} \Pi_{u}$ series can be efficiently populated. The $\mathrm{Q}_{1}$ states decay into the ground state of the ion, $1 \mathrm{~s} \sigma_{g}$, leading to nuclear fragments with 2-7 eV, while the $\mathrm{Q}_{2}$ states can decay into both the $1 \mathrm{~s} \sigma_{g}$ and $2 \mathrm{p} \sigma_{u}$ ionization continua. All these channels interfere giving rise to structures in the nuclear kinetic energy distributions ${ }^{27,301,388}$ and, more importantly, to asymmetric angular distributions of the ejected electron and localization of the remaining 
electron. Remarkably, the magnitude and directionality of both the asymmetry and the localization depend on the pump-probe delay. Both phenomena are the result of the coherent superposition of states of different parity but with identical energy of the fragments and angular momentum of the ejected electron. The coherent superposition can only be achieved in the presence of the IR field and is observed for kinetic energy of the fragments around 2-8 eV. Two different mechanisms were identified as the origin of electron asymmetry and localization: i) the interference between the autoionization decay into the $1 \mathrm{~s} \sigma_{g}$ state and direct ionization through the $2 \mathrm{p} \sigma_{u}$ continuum resulting from the simultaneous absorption of a EUV and an IR photon, and ii) the time-delayed absorption of an IR photon by the ion left behind upon ionization by the EUV pulse (see panels on the right hand side of Fig. 25). In either case, the final wave function can be approximately written as the coherent sum of the following contributions,

$$
\begin{aligned}
\Psi(t) & =c_{1}(t)\left[1 s \sigma_{g}(1) \epsilon l_{g}(2)\right]_{g}+c_{2}(t)\left[1 s \sigma_{g}(1) \epsilon l_{u}(2)\right]_{u}+ \\
& +c_{3}(t)\left[2 p \sigma_{u}(1) \epsilon l_{u}(2)\right]_{g}+c_{4}(t)\left[2 p \sigma_{u}(1) \epsilon l_{g}(2)\right]_{u}
\end{aligned}
$$

where we have simplified the notation by ignoring the antisymmetrization with respect to electrons 1 and 2, and the ionized electron 2 is described by a function of energy $\epsilon$ and angular momentum $l_{u}\left(l_{g}\right)$. Note that the corresponding ionization amplitudes $\left(c_{1}, c_{2}, \ldots\right)$ are complex numbers that explicitly depend on the time-delay between the pulses and will evolve with their stationary phases once both pulses are gone. ${ }^{32}$ The localization of the remaining electron on the right or the left nucleus in the laboratory frame is theoretically obtained by projecting the final wave function into the localized states defined as:

$$
\begin{aligned}
& \Psi_{L}=\left[1 s \sigma_{g}(1)+2 p \sigma_{u}(1)\right] \epsilon l_{g / u}(2) \\
& \left.\Psi_{R}=\left[1 s \sigma_{g}(1)-2 p \sigma_{u}(1)\right]\right] \epsilon l_{g / u}(2)
\end{aligned}
$$


where $\Psi_{L}$ has the bound electron localized on the left proton and $\Psi_{R}$ on the right one. The asymmetry is then defined as the difference between the probabilities to localize the proton on the left $\left(N_{L}\right)$ and on the right $\left(N_{R}\right)$ :

$$
N_{L}-N_{R}=\sum_{i=g, u}\left[\left|\left\langle\Psi_{L, i} \mid \Psi(t)\right\rangle\right|^{2}-\left|\left\langle\Psi_{R, i} \mid \Psi(t)\right\rangle\right|^{2}\right]=4 R e\left[c_{1} c_{4}^{*}+c_{2} c_{3}^{*}\right]
$$

The above expression indicates that a laboratory-frame asymmetry arises as long as $c_{1}, c_{4} \neq 0$ or $c_{2}, c_{3} \neq 0$, i.e., provided that there is a superposition of states that involves ionic states of different parity and a continuum electron with the same angular momentum. In Fig. 25, the calculated asymmetry parameter, obtained from an accurate solution of the TDSE, is plotted as a function of the proton kinetic energy and the time-delay between the pulses. A very good agreement was found between experimental and theoretical data. Distinct asymmetry fringes can be observed for delays up to $7 \mathrm{fs}$ and at proton kinetic energies between $\approx 5-7$ $\mathrm{eV}$, where both direct photoionization through the $2 \mathrm{p} \sigma_{u}$ channel and autoionization through the $1 \mathrm{~s} \sigma_{g}$ channel are possible. The asymmetry parameter oscillates with half the periodicity of the IR probe pulse. While the interaction with the attosecond pulse can only lead to molecular states of ${ }^{1} \Sigma_{u}^{+}$symmetry (corresponding to the ionization amplitudes $c_{2}$ and $c_{4}$ in Eq. 85), the interaction with the IR field makes $c_{1}$ and $c_{3}$ non-zero through the two mechanisms mentioned above. Figure 25 shows that the asymmetry fringes are smoother after 3-4 fs, which is the lifetime of the lowest $Q_{1}$ autoionizing state. This can be explained as follows. Before autoionization takes place, the IR pulse drives the electron from one side of the molecule to the other, still in the presence of the second electron, which has not left the molecule yet and, therefore, can perturb this motion. In contrast, at longer delays, the first electron is gone, and the IR pulse can drive the motion of the remaining electron without any interference with the other electron. As a consequence, the oscillations are smoother.

In summary, this work showed that electron localization following dissociative ionization of the $\mathrm{H}_{2}$ molecule can be controlled by using an appropriate timing of the EUV pump and 
IR probe pulses. Furthermore, from the shape of the asymmetry fringes one can directly measure the lifetime of the autoionizing states, something that is not always obvious in molecules when electron and nuclear dynamics are strongly coupled.

Shortly after this work was published, trains of attosecond EUV pulses were employed to singly ionize $\mathrm{D}_{2}$ and investigate the role of nuclear motion in the induced electron dynamics ${ }^{389}$ following a scheme similar to RABBITT (see section 3.1.1). The results showed that nuclear motion introduces a laser-induced coupling between ionic states that depends on time. As a consequence, the variation of the measured photoelectron spectrum with the pump-probe delay is different from that obtained from RABBITT measurements in atomic systems. A few years later, Ranitovic et $\mathrm{al}^{390}$ extended this pump-probe scheme by using trains of VUV attosecond pulses in combination with a phase locked IR pulse as a pump. The chosen VUV wavelengths ensured that excitation (not ionization, as in the previous examples) of the $\mathrm{D}_{2}$ molecule was the dominant channel. In the experiment, three different attosecond VUV harmonic combs were used, leading to population of the $\mathrm{B}^{1} \Sigma_{u}^{+}$and $\mathrm{EF}{ }^{1} \Sigma_{g}^{+}$states through different one- and two-photon pathways that involve the 7th and 9th harmonics of the EUV train and the phase locked IR field. Each harmonic comb was used to create a different superposition of electronic and vibrational states, i.e., a different vibronic wave packet, which evolves in the potential energy curves of the $\mathrm{B}^{1} \Sigma_{u}^{+}$and $\mathrm{EF}{ }^{1} \Sigma_{g}^{+}$states covering different regions of the internuclear distance. By using this scheme, the authors could control tunneling of the nuclear wave packet through the barrier of the double-well potential energy curve of the $\mathrm{EF}^{1} \Sigma_{g}^{+}$state and induce selective bond-breaking as well as control of the ionization yields by ionizing at different times.

\subsubsection{EUV-pump/EUV-probe}

As explained in section 3.2.3, recent experimental efforts devoted to increase the intensity of EUV pulses produced from HHG pursue the realization of EUV-only pump-probe experiments. In contrast with IR fields, EUV pulses barely distort the molecular potential due to 
their short wavelength (Keldysh parameter much larger than 1), so that they are in principle more appropriate to access intrinsic molecular dynamics. For many years, the intensities of the EUV pulses generated in high harmonic emission were too low to successfully perform such pump-probe experiments. However, as previously mentioned, for more than a decade FELs had been able to generate high peak power pulses with durations of few femtoseconds in this photon energy region. ${ }^{180,391,392}$ For this reason, the first attempts to perform EUVpump/EUV-probe experiments made use of ultrashort pulses generated in FELs, namely to investigate ultrafast dynamics first in the molecular ions $\mathrm{H}_{2}^{+}$and $\mathrm{D}_{2}^{+393,394}$ and then in $\mathrm{N}_{2}$ and $\mathrm{O}_{2},{ }^{244}$ and in acetylene. ${ }^{243,395}$ In the work by Jiang et al, ${ }^{393,394}$ twin EUV pulses of a few tens of femtoseconds generated in the FEL facility in Hamburg (FLASH) were used in a pump-probe experiment with a time resolution slightly below 10 fs. The nuclear wave packet dynamics associated with the ground state of the singly ionized $\mathrm{D}_{2}^{+}$molecule was tracked in time by its direct projection onto the Coulomb explosion channel. In practice, this was done first by ionizing neutral $D_{2}$ molecules with the EUV pump pulse and then by ionizing the remaining $\mathrm{D}_{2}^{+}$molecular cation with the EUV probe pulse. By measuring energy resolved ionization yields as a function of the pump-probe delay, these authors were able to separate contributions from direct and sequential two-photon double ionization processes, and infer from the sequential channel the nuclear dynamics induced by the pump pulse in the $\mathrm{D}_{2}^{+}$ion. The key point that allowed for this separation was the use of REMI (or COLTRIMS) techniques, which allows one to measure in coincidence the energy of all ejected charged fragments following the Coulomb explosion that results from the removal of the two electrons.

In order to go a step further and track not only the ultrafast motion of the nuclei, but also that of electrons, shorter and better controlled pulses are required. The FEL community has advanced in the last few years in this direction and pulses as short as 5 fs ${ }^{249,396}$ or even spikes with sub-femtosecond duration ${ }^{250}$ have been produced. Moreover, they have incorporated attosecond techniques in order to characterize the temporal profile of these short pulses and 
make them suitable for pump-probe experiments with ultrahigh time resolution. ${ }^{249,396-398}$ This has been demonstrated in a recent work at FERMI, where two-color femtosecond EUV pulses have been produced with a controlled variable delayed phase ${ }^{399}$ and used to capture the dynamics of the $4 \mathrm{~s}$ resonance of Ne with a temporal resolution of around 3 attoseconds! For this, the experiment made use of a VMI spectrometer to measure the angular distribution of the ejected electron from which the resonant state dynamics was extracted.

The advances in FEL facilities are, therefore, very promising regarding future EUV-EUV experiments. Nevertheless, optimal attosecond pump-probe schemes require even shorter pulses, in the sub-fs regime, such that one can actually track electronic wave packets in the absence of any external field, e.g., in between the pump and probe pulses. Such limitations can be overcome by using EUV pulses produced from HHG. In the last few years, the intensities that one can reach with such pulses have significantly increased, ${ }^{207,400}$ making the realization of EUV-only pump-probe experiments possible. Using the experimental setup described in Sect. 3.2.3, the first EUV-pump/EUV-probe experiment with attosecond pulses resulting from HHG was performed in the Xe atom by Tzallas et al. ${ }^{207}$ The same experimental setup and time resolution were later used in $\mathrm{H}_{2}$ by Carpeggiani et al, ${ }^{210}$ who performed the first attosecond EUV-pump/EUV-probe measurements in a molecule. In this case, a 150-nm-thick indium filter was used, selecting an energy range around 12.5-18 eV, such that an electronic wave packet is created in the neutral molecule and then probed in the single ionization continua. The scheme and the energetics are shown in Fig. 26. The broad energy bandwidth of the single attosecond pulse leads to one-photon excitation of a manifold of electronic states of the molecule and the associated vibrational states that have a favorable Franck-Condon overlap with the ground vibrational state of the neutral $\mathrm{H}_{2}$ molecule. The ionization potential of $\mathrm{H}_{2}$ is $15.4 \mathrm{eV}$ for the non-dissociative channel $\left[\mathrm{E}\left(\mathrm{H}_{2}+(\mathrm{v}=0)\right)-\mathrm{E}\left(\mathrm{H}_{2}(\mathrm{v}=0)\right]\right.$ and $18.15 \mathrm{eV}$ for the dissociative one $\left[\mathrm{E}\left(\mathrm{H}^{+}+\mathrm{H}(\mathrm{n}=1)\right)-\mathrm{E}\left(\mathrm{H}_{2}(\mathrm{v}=0)\right]\right.$. Consequently, the dissociative single ionization channel is only accessible by two-photon absorption. Therefore, this ionization channel can be used as the observable to probe the coupled electron and nuclear dynamics 

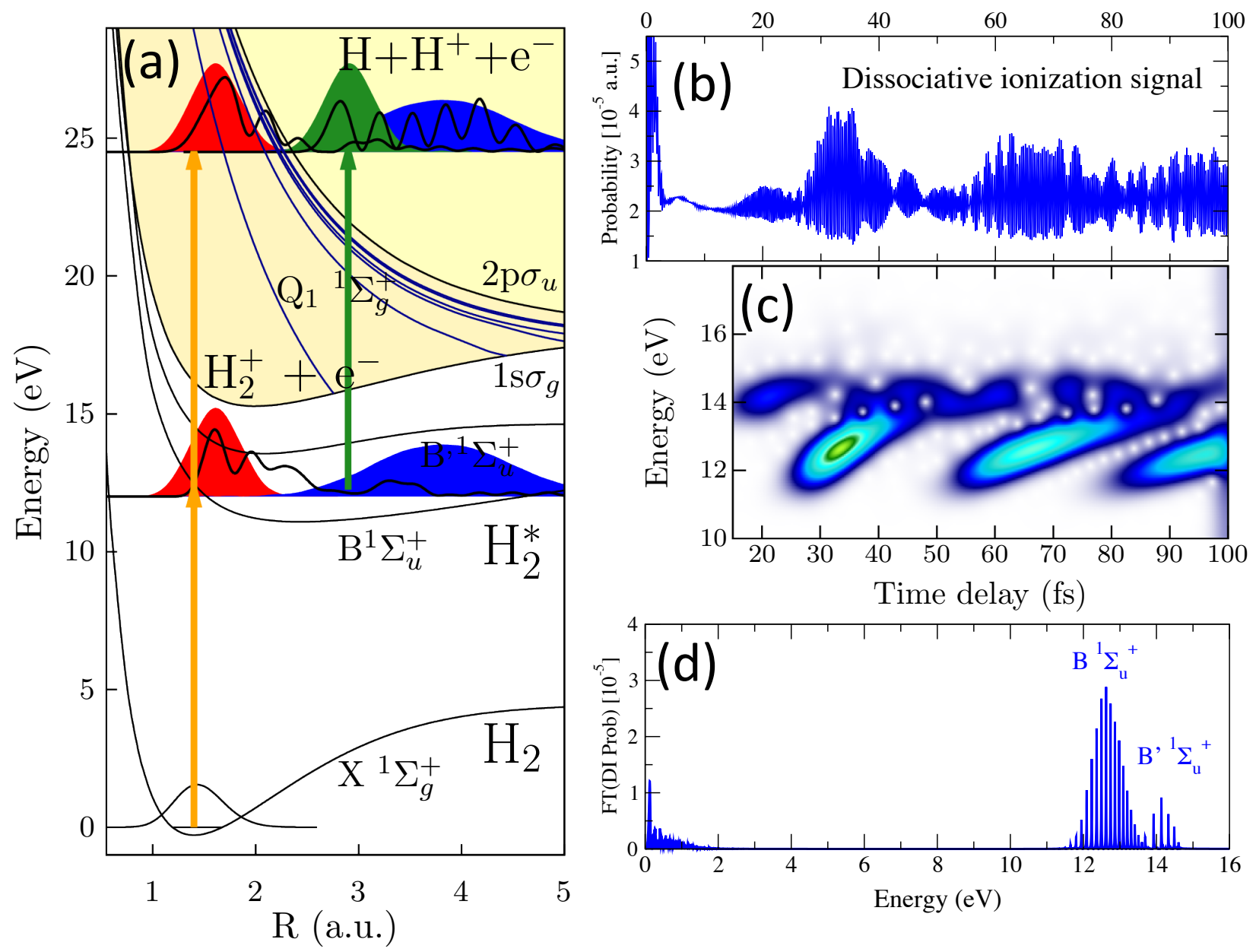

Figure 26: (a) Schematic representation of the EUV pump/EUV probe scheme, with two pulses of 2 fs duration with a time delay among them. The relevant potential energy curves of the $\mathrm{H}_{2}$ molecule are included: the ground state $\left(\mathrm{X}^{1} \Sigma_{g}^{+}\right)$, the two lowest excited states $1^{1} \Sigma_{u}^{+}$(B and B'), the lowest $\mathrm{Q}_{1}{ }^{1} \Sigma_{g}^{+}$doubly excited states, and the two lowest ionization thresholds. The blue shaded areas represent the squares of the WP generated by the pump pulse after evolving in time. The red shaded areas represent the WPs generated by the probe pulse (replicas of the blue ones prior evolution). The orange arrows represent direct two-photon ionization by the pump and probe pulses, and the green arrow represents the absorption of a single photon from the probe pulse after free evolution of the WP generated by absorption of a single photon from the pump pulse (sequential two-photon ionization process). The WP created in the sequential process is represented by the green shaded area. The black curves superimposed on the blue and red WPs in C represent the WP resulting from the interference between the red and the blue WPs. (b) Dissociative ionization yield as a function of the time delay. (c) Short-time and (d) standard Fourier transform of the dissociative ionization yield. 
induced by the pump pulse in the excited molecule. In the experiment, the one-photon absorption path is demonstrated to be a minor channel in the dissociative ionization by looking at the slope of the ionization signal with respect to the pulse intensity, which is close to 2 in a log-log scale. In contrast, it dominates the non-dissociative ionization channel, as indicated by a measured slope close to 1 . Furthermore, for photon energies within the bandwidth of the pulse, a two-photon resonant transition at the equilibrium internuclear distance of the molecule only reaches the $1 \mathrm{~s} \sigma_{g}$ ionization limit, as shown in Fig. 26. As the nuclear wave packet evolves and reaches larger internuclear distances, the second ionization threshold $2 \mathrm{p} \sigma_{u}$ opens, leading to ionization with non-zero energy protons. Due to the repulsive character of the $2 \mathrm{p} \sigma_{u}$ state, the proton energy distribution directly maps the evolution of the pumped wave packet. ${ }^{401}$ The experiment cannot distinguish those channels a priori, but it does measure the proton signal as a function of the pump-probe delay and distinguishes protons with kinetic energy close to zero from those with non-zero kinetic energy. The total proton yield oscillates with time, capturing the electronic beatings between the B and B' ${ }^{1} \Sigma_{u}^{+}$ and $\mathrm{C}$ and $\mathrm{D}^{1} \Pi_{u}$ states within the pumped wave packet. Those frequency differences can be clearly distinguished in the Fourier transform spectra. The experimental results present a good agreement with ab initio theoretical calculations. The latter are shown in Fig. 26(b) for molecules parallel to the polarization axis.

The above experiment thus opens the way to further studies in more complex molecules. However, progress into this direction also requires a full characterization of the CEP of these pulses. Indeed, as theoretically demonstrated in, ${ }^{402}$ application of the same EUVpump/EUV-probe scheme in $\mathrm{H}_{2}$ by using phase-stabilized pulses creates a molecular interferometer that results from the direct and sequential two-photon ionization processes illustrated in Fig. 26. The two-photon direct channel that is used as a reference is always present and interfere with the time-delayed sequential process that maps the evolving wave packet. The interferences between these two paths lead to oscillating patterns in the total and energy-differential ionization yields that allow one (i) to disentangle the electronic and 
nuclear dynamics generated in the excited molecule, and (ii) to capture the autoionization dynamics in the final state. Note that the ionization signal also reflects the optical interference resulting from using two identical pulses, i.e. the interference between the identical wave packets generated by the pump and probe pulses by direct two-photon ionization. Despite the apparent complexity of the problem, a simple time-frequency analysis of the ionization yields, analogous to that performed in the PROOF technique described in section 3.1.2, can already provide the amplitude and phase information in order to fully reconstruct the molecular (electronic and nuclear) wave packet created by the pump pulse. ${ }^{402,403}$ Figure 26(b) shows the dissociative ionization yield as a function of the pump-probe delay for a simulation using two identical pulses of 2 fs duration, $10^{12} \mathrm{~W} / \mathrm{cm}^{2}$ intensity and centered at $14 \mathrm{eV}$. A very fast oscillation (with a $\approx 350$ as period) appears embedded in a slower oscillation (28-30 fs). A time-frequency analysis of these results is provided by the time-window Fourier transform of the yield shown in Fig. 26(c), obtained by using a Gaussian shape 2 -fs window. In the $y$-axis, we can distinguish the contribution from two different electronic states, the B and B' states around 12 and $14 \mathrm{eV}$, respectively. Meanwhile, the x-axis uncovers the average vibrational periods of the nuclear wave packet components associated with them. One could then select a given time delay to favor the transition through a preferred electronic state. Figure 26(d) shows the standard Fourier transform, which also includes the contribution from lower energies. The observed peaks correspond to beatings, i.e., energy differences, between vibronic states associated either with the B or the B' states, or with one of each other. ${ }^{402}$ It is worth noticing the amazingly large amount of information that is encoded in the total ionization signal. To extract it in a real experiment, the use of multi coincidence detection devices, such as REMI, is thus highly desirable. With this, one can reasonably expect that a full reconstruction of the complete vibronic wave packet generated in the $\mathrm{H}_{2}$ molecule will be realistically achieved in the near future. Also, as forthcoming attosecond EUV/EUV experiments are expected to be performed in more complex molecular targets, see e.g., ${ }^{404}$ it is very likely that reconstruction methods as that described above or 
extensions of it will be soon employed to investigate ultrafast electron and nuclear dynamics in molecules.

\subsection{Beyond $\mathrm{H}_{2}$ : electron dynamics in more complex diatomics and small polyatomics}

These pioneering works on $\mathrm{H}_{2}$ opened the way to investigate electron and nuclear dynamics in many-electron diatomic and small polyatomic molecules. In the case of diatomics, most experimental and theoretical efforts have concentrated on the $\mathrm{O}_{2}, \mathrm{CO}$ and $\mathrm{N}_{2}$ molecules. For example, attosecond pulse trains combined with NIR femtosecond probe pulses have been used to investigate dissociation dynamics and vibrational motion in the $\mathrm{O}_{2}^{+}$molecular cation, ${ }^{405,406}$ and narrow-band EUV excitation has been exploited to study autoionization in $\mathrm{O}_{2} \cdot{ }^{407}$ Also, waveform-controlled high-intensity NIR pulses have been used to investigate ultrafast electron dynamics in CO. ${ }^{408}$ By combining attosecond pulse trains with VMI spectroscopy, the autoionization dynamics of $\mathrm{N}_{2}$ has been recently investigated. ${ }^{409}$ In these experiments, autoionization was temporally resolved by mapping the internuclear distance at which the $\mathrm{N}_{2}^{+}$ions resulting from autoionization begin to dissociate. This has been shown to be a precise clock that provides femtosecond temporal resolution. Dissociative ionization of $\mathrm{N}_{2}$ has been further studied by using femtosecond time-resolved photoelectron and photoion spectroscopy. 410

When broadband attosecond pulses are used to irradiate a molecule containing many electrons, even a small one like $\mathrm{N}_{2}$, the coherent superposition of states that results from such excitation process usually involves a large number of electronic states. These states must represent the possibility that an electron is ejected from different molecular orbitals but also that the electrons remaining in the molecular cation may be excited through a shake-up mechanism. As a consequence, disentangling the electronic and nuclear dynamics that follows ionization by the attosecond pulse is a real challenge. For this reason, experimental efforts need the guide and support from theoretical simulations that provide a precise description of 

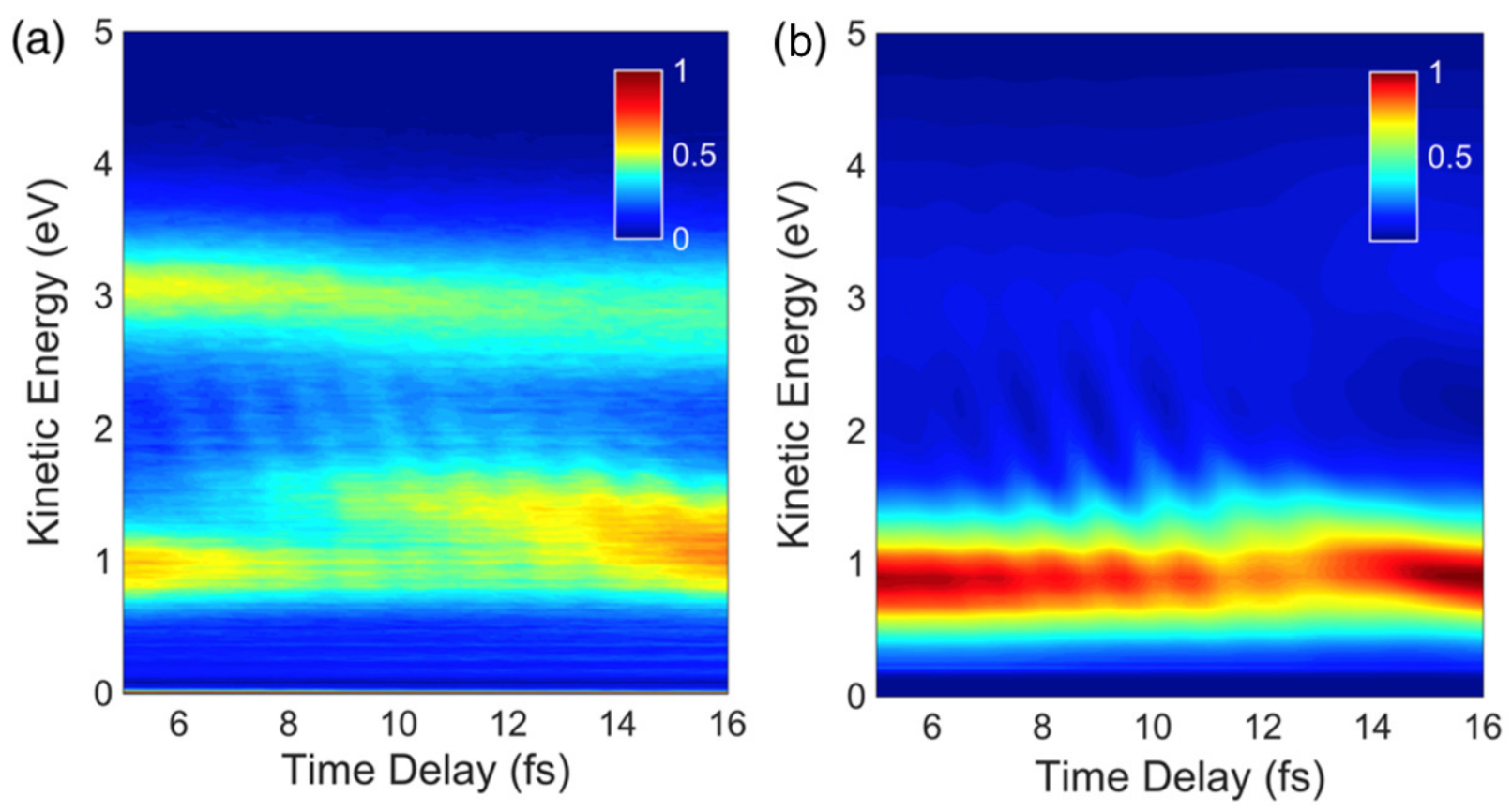

Figure 27: (a) Time-dependent $\mathrm{N}^{+}$kinetic energy spectra acquired within the pump-probe delay interval 5-16 fs. (b) Simulation of time-dependent $\mathrm{N}^{+}$kinetic energy spectra in the same delay interval. From ref. 177. Copyright 2015 American Physical Society.

the ionization amplitudes as well as of the potential energy curves (PECs) and corresponding non-adiabatic and laser-induced couplings, by including all electrons of the system. An example of how theory and experiment nicely complement each other has been recently reported by Trabattoni et al. ${ }^{177}$ In this work, the authors show that isolated attosecond pulses can be exploited to image the dissociative ionization dynamics of $\mathrm{N}_{2}$ with extremely high temporal resolution and, with the help of theory, that precise information on the shape of the PECs involved in the dissociative mechanism can be extracted. In the experiment, molecular nitrogen was photoionized by the combination of a sub-300-as attosecond pump pulse (spectral range between $16 \mathrm{eV}$ and $50 \mathrm{eV}$ ) and a waveform-controlled sub-4-fs NIR probe pulse. As for the experiment performed in, ${ }^{409}$ the 3D momentum distribution of the $\mathrm{N}^{+}$atomic ions was measured with VMI as a function of the delay between the pump and probe pulse. Figure 27(a) shows the kinetic energy spectrum of $\mathrm{N}^{+}$, obtained by integration of the angular distribution over a small angle along the laser polarization axis, in the pump- 

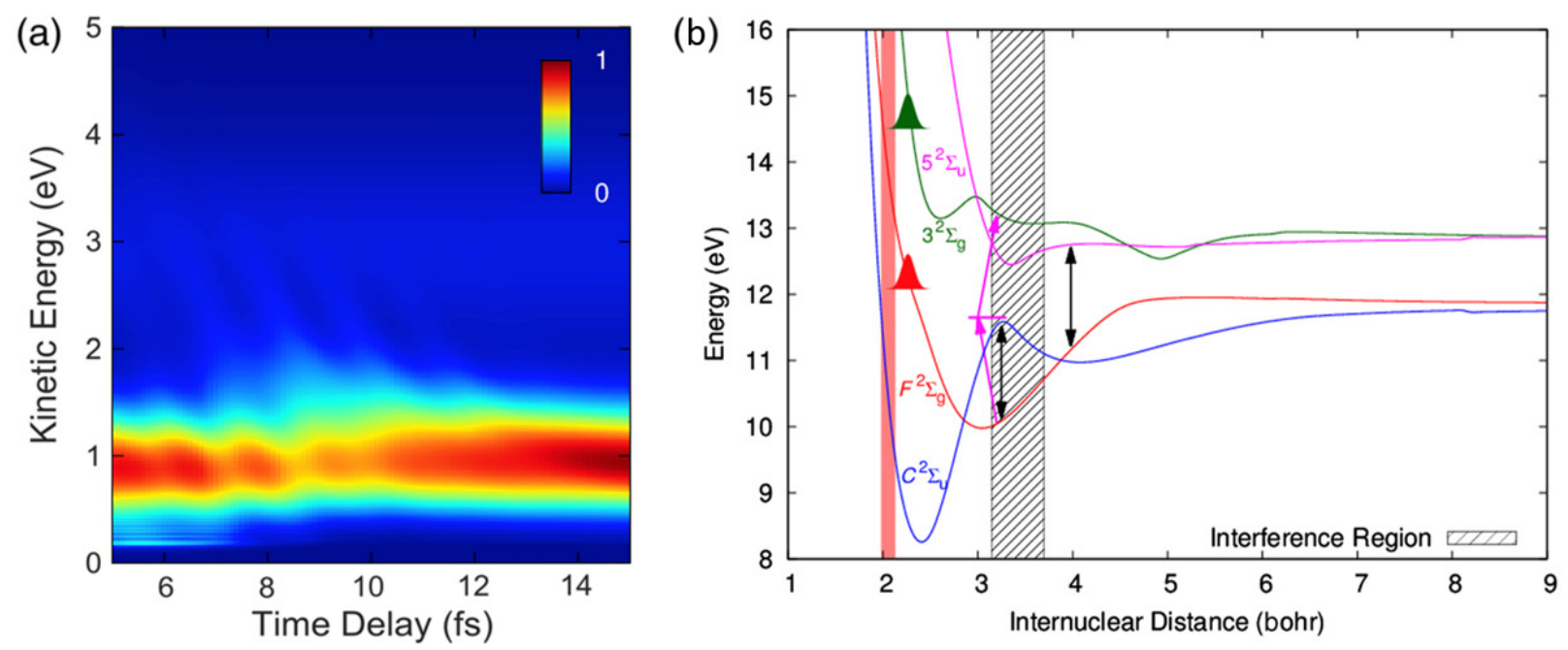

Figure 28: (a) Simulation of time-dependent $\mathrm{N}^{+}$kinetic energy spectra with the simplified model. (b) Four-state model, where the $\mathrm{F}^{2} \Sigma_{g}, 3^{2} \Sigma_{g}, \mathrm{C}^{2} \Sigma_{u}$ and $5^{2} \Sigma_{u}$ states were considered. From ref. 177. Copyright 2015 American Physical Society.

probe delay range between $5 \mathrm{fs}$ and $16 \mathrm{fs}$. Two main features can be observed: (i) the depletion of the signal at $1 \mathrm{eV}$ occurring $8 \mathrm{fs}$ after the zero delay, (ii) the appearance of a fast modulation in the same delay range, with a periodicity of $1.22 \mathrm{fs}$. Furthermore, the modulation shows an energy-dependent phase, resulting in a tilt of the fringes. The origin of these features was understood by solving the TDSE in a large basis of diabatic $\mathrm{N}_{2}^{+}$ states and by taking into account the couplings induced by the NIR probe pulse. Figure 27(b) shows the calculated $\mathrm{N}^{+}$kinetic energy spectrum as a function of the pump-probe delay, obtained by using the same laser parameters as in the experiment. As can be seen, the agreement between theory and experiment is very good in the low-energy region of the spectrum where single ionization is the dominant process (the upper band in the experimental spectrum corresponds to double ionization of the molecule, which could not be described by the theoretical simulations since $\mathrm{N}_{2}^{2+}$ states were not included in the basis set used to solve the TDSE). In spite of the reasonable agreement, the full calculation itself does not provide too much information about the underlying mechanisms. However, it can be used to identify the minimum number of states responsible for the observed dynamics. These 
are the $\mathrm{F}^{2} \Sigma_{g}, 3^{2} \Sigma_{g}, \mathrm{C}^{2} \Sigma_{u}$ and $5^{2} \Sigma_{u}$ states of $\mathrm{N}_{2}^{+}$. Figure $28(\mathrm{a})$ shows the result of a model calculation that only include these four states. As can be seen, the main features observed in the experiments are qualitatively reproduced by the model. From this model, the physical mechanisms leading to the observed experimental features are schematically represented in Fig. 28(b): the depletion of the signal around $1 \mathrm{eV}$ is related to resonant single-photon transitions from the $\mathrm{F}^{2} \Sigma_{g}$ state to the $5^{2} \Sigma_{u}$ state and from the $\mathrm{F}^{2} \Sigma_{g}$ state to the $\mathrm{C}^{2} \Sigma_{u}$ state induced by the NIR probe pulse (black double-headed arrows in the figure). The measured delay of 8 fs corresponds to the time required by the nuclear wave packet (NWP) to reach the single-photon transition point. Additionally, the ultrafast periodic modulation results from the interference between the initial population of the $3^{2} \Sigma_{g}$ state and the population transferred to the $3^{2} \Sigma_{g}$ state from the $\mathrm{F}^{2} \Sigma_{g}$ state via a two-photon transition using the $5^{2} \Sigma_{u}$ state as a virtual intermediate state (magenta single-headed arrows). The tilt in the fringes is due to the dispersion of the components of the NWP while traveling along the PECs. This time-vs-energy dependence carries crucial and precise information about the quantum path followed by the NWP, namely the shape of the potential energy curves involved in the interference. Trabattoni et al have shown that by artificially changing the slope of the $3^{2} \Sigma_{g}$ state the resulting tilt in the fringes dramatically changes and the agreement with the experimental data is lost. This work demonstrates that the sub-femtosecond temporal resolution provided by attosecond experiments, when applied to many-electron diatomic molecules, provide a stringent test of elaborate theoretical methods, which are necessary to uncover the mechanisms behind the observed dynamics.

Recently, Neidel et al ${ }^{178}$ have reported the observation of attosecond electron dynamics in $\mathrm{N}_{2}$ and in the small polyatomic molecules $\mathrm{CO}_{2}$ and $\mathrm{C}_{2} \mathrm{H}_{4}$. In this experiment, the timedependent dipole induced by a NIR laser field was probed by using an EUV attosecond pulse train (APT). Atomic and molecular cations produced by EUV-induced ionization were recorded as a function of the delay between the EUV APT and the NIR pulse, which was phase locked to the EUV APT. Figure 29(c) shows the time-of-flight spectra recorded with 


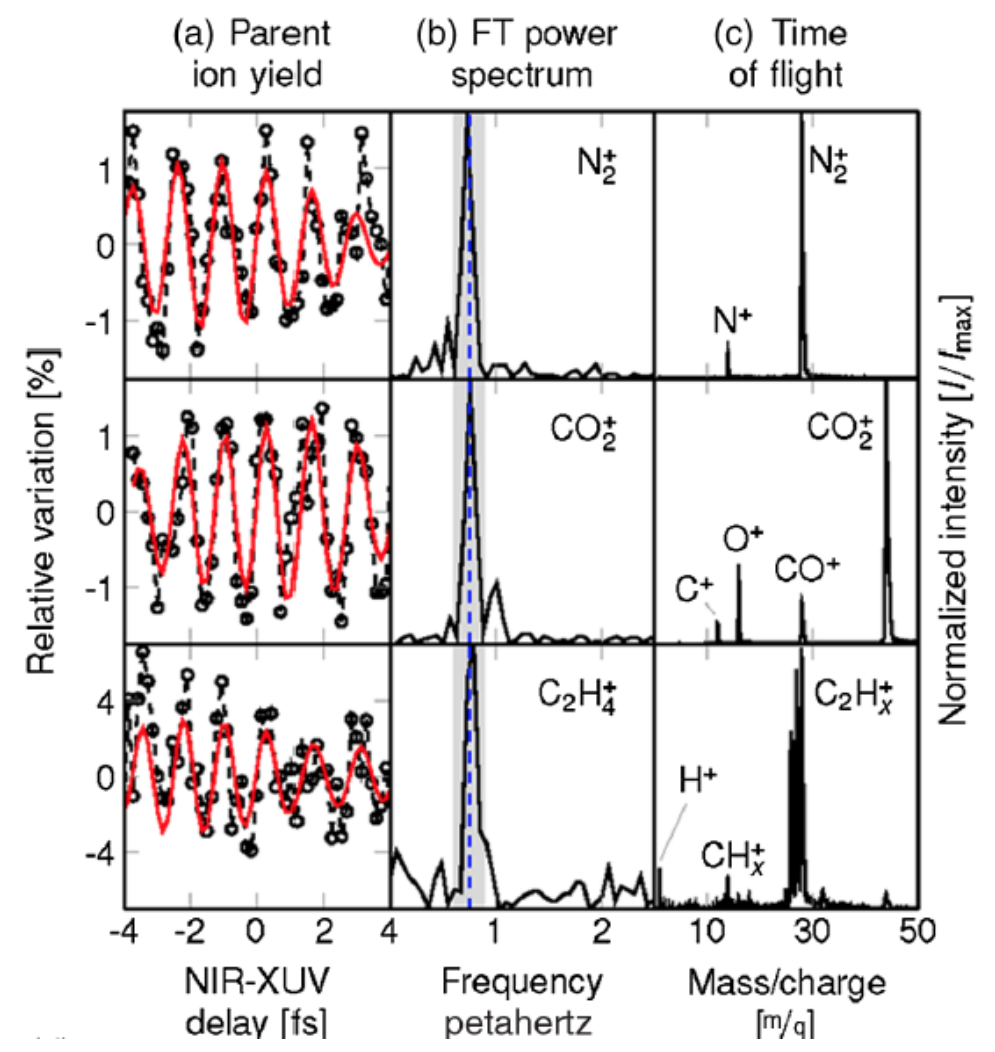

Figure 29: (a) $\mathrm{N}_{2}^{+}, \mathrm{CO}_{2}^{+}$and $\mathrm{C}_{2} \mathrm{H}_{4}^{+}$yields (black dashed curves) as a function of the delay between the NIR pump and the EUV APT probe. (b) Normalized Fourier transform (FT) power spectra of the yields shown in panel (a). The shaded gray area was used in an inverseFT, leading to the red solid curves in the left panel. The parent ionization yields oscillate with a period corresponding to half the period of the NIR field (dashed blue line). (c) Timeof-flight spectra obtained for ionization of $\mathrm{N}_{2}, \mathrm{CO}_{2}$ and $\mathrm{C}_{2} \mathrm{H}_{4}$ molecules by the EUV APT. In each molecule the abundance of the parent ion dominates over that of the ionic fragments. From ref. 178 with permission. Copyright 2013 American Physical Society.

velocity map imaging detectors after interaction with the APT only. In $\mathrm{N}_{2}$, the ionization leads mainly to the formation of an intense parent ion peak and a small fraction of atomic $\mathrm{N}^{+}$ions. The latter are formed because, at the EUV photon energies used in the experiment, predissociative as well as dissociative states are accessible. In $\mathrm{CO}_{2}$, more charged fragments are observed, although, as in the previous case, the dominant contribution comes from the $\mathrm{CO}_{2}^{+}$parent ion. Even more fragmentation channels are observed in $\mathrm{C}_{2} \mathrm{H}_{4}$, since many more states are involved in the ionization and dissociation processes, but again the signal 
corresponding to the parent ion dominates the mass spectrum. The variation of the singlycharged parent molecular ion yields with pump-probe delay are shown in Fig. 29(a) and the corresponding Fourier transform power spectra in Fig. 29(b). The latter clearly show that the time variation of the yields is explained by a single frequency component. For $\mathrm{N}_{2}^{+}$ions, the amplitude of the oscillation is approximately $1-2 \%$ of the total signal and the period is equal to half the NIR laser period. Similar in-phase oscillations were observed for $\mathrm{N}^{+}$ions, thus showing that the total ionization yield also oscillates with the same periodicity. From this observation, one can thus conclude that the NIR field polarizes the neutral $\mathrm{N}_{2}$ molecule and that the transition induced by the APT is sensitive to the redistribution of the electronic density. For $\mathrm{CO}_{2}$ and $\mathrm{C}_{2} \mathrm{H}_{4}$ the amplitude of the oscillations is larger, around $2.5 \%$ and $6 \%$, respectively, reflecting the larger polarizability of these molecules: $1.71 \AA^{3}$ for $\mathrm{N}_{2}, 2.507 \AA^{3}$ for $\mathrm{CO}_{2}$, and $4.18 \AA^{3}$ for $\mathrm{C}_{2} \mathrm{H}_{4}$. These findings were confirmed by the results of TDDFT simulations reported in the same work. Therefore, this experiment proves that NIR-driven time-dependent dipoles in neutral molecules can be observed by monitoring variations of the EUV-induced ionization yields and that the amplitude of the observed oscillations is proportional to the magnitude of the molecule's polarizability. As a consequence this can be seen as the first implementation of molecular Stark spectroscopy on the attosecond time scale.

Electron dynamics has also been investigated by means of high harmonic spectroscopy. In a recent joint experimental and theoretical work by Kraus et al, ${ }^{188}$ attosecond charge migration has been measured and controlled in ionized iodoacetylene ( $\mathrm{HCCI}^{+}$) by analyzing the harmonic light emitted after irradiation of HCCI with a strong IR pulse. In this molecule, strong field ionization can leave the cation in more than one electronic state, namely the $\tilde{X}^{+2} \Pi$ and $\tilde{A}^{+2} \Pi$ ones. These states are strongly coupled by the IR field, thus leading to the formation of an electronic wave packet and hence to charge migration. Interestingly, the coupling between these states depends on molecular orientation, which therefore allows one to modify the charge migration process by using different molecular alignments. In the 
experiment, control over the spatial orientation of the molecule was achieved by using one phase-controlled two-color (800 nm $+400 \mathrm{~nm}$ ) laser pulse for impulsive orientation. The fixed-in-space ensemble of molecules was then interrogated by a high-harmonic generation (HHG) pulse one rotational period (157.0 ps) later. By considering different orientations, the authors could separately reconstruct field-free and laser-driven charge migration. Indeed, for molecules aligned perpendicular to the laser polarization direction, the effect of the IR field on charge migration was found to be negligible, while for parallel molecules, the IR field was able to induce substantial population transfer between the $\tilde{X}^{+2} \Pi$ and $\tilde{A}^{+2} \Pi$ states, which depends on the head-to-tail orientation of the molecule. As explained in section 2.1, the time resolution arises from the subcycle nature of the HHG process. Thus, every emitted harmonic order could be associated with a unique traveling time of the electron wave packet in the continuum by experimentally selecting the short electron trajectories. The reconstruction of charge migration became possible from the measurement of the harmonic peak intensities and phases as functions of the orientation angle. This was done by performing a numerical inversion procedure based on the generalized theory of HHG presented in section 4.4.

The results of such reconstruction are shown in Fig. 30 for the case of molecules perpendicularly oriented with respect to polarization direction of the field. As can be seen, the strong-field ionization creates a one-electron hole localized at the iodine side of the molecule, compatible with the low ionization potential and high polarizability of the iodine atom. Subsequently, the hole delocalizes over the molecule and then localizes at the acetylene side after 930 as. Because in this particular case the differential Stark shift of the $\tilde{X}^{+2} \Pi$ and $\tilde{A}^{+2} \Pi$ levels amounts to only $18 \mathrm{meV}$ and laser induced population transfer between these states is not possible by symmetry, the reconstructed dynamics corresponds to quasi-field-free charge migration. Reconstruction of charge dynamics for other molecular orientations and/or by using different laser wavelengths was also achieved. In particular, a strong dependence of the dynamics was observed by changing the IR wavelength from $800 \mathrm{~nm}$ to $1300 \mathrm{~nm}$.

This work has shown several important aspects of the preparation and control of charge 


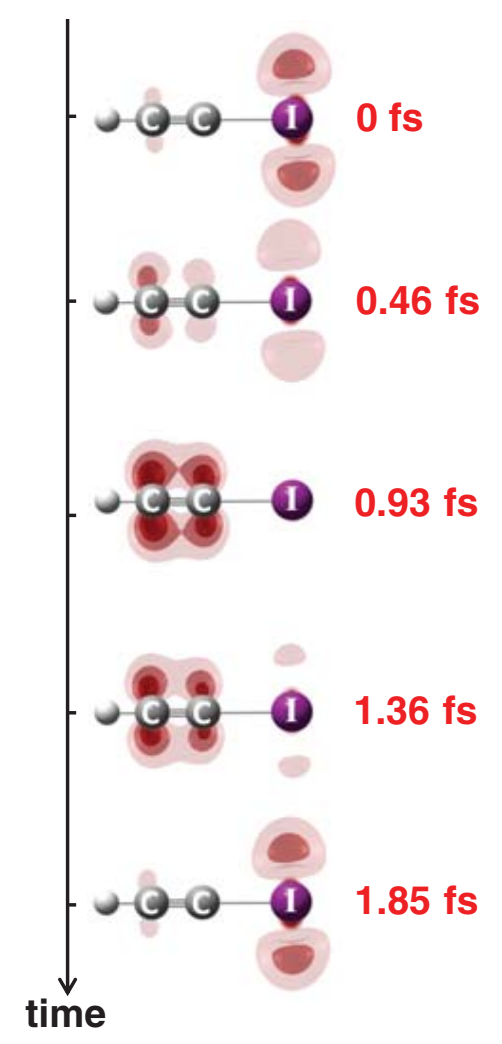

Figure 30: Reconstructed quasi field-free electron dynamics in $\mathrm{HCCI}^{+}$as a function of time after ionization. Molecules are perpendicularly oriented with respect to the polarization direction of the laser field. The hole, initially localized at the iodine side of the molecule, first delocalizes and then localizes at the acetylene site after 930 as. From ref. 188 with permission. Copyright 2015 The American Association for the Advancement of Science (AAAS).

migration induced by intense laser pulses by using high harmonic spectroscopy as analysis tool. On the one hand, the initial phase of the hole wave packet strongly depends on the orientation of the molecule with respect to the ionizing laser field, thus suggesting that both molecular alignment and orientation are necessary for a well-characterized measurement and can be used as control parameters. On the other hand, the laser field can be used to achieve control over charge migration, especially when the relevant transition dipole moments are large and the level separations are small, resulting in strongly nonadiabatic dynamics. 


\section{Towards sub-femtosecond electron dynamics in bio-relevant molecules}

Time-resolved studies of electron dynamics in bio-relevant molecules may shed light on the underlying mechanisms in a variety of fundamental processes such as photosynthesis, cellular respiration or electron transport along large peptides and proteins. ${ }^{411-413}$ Electron dynamics of these processes are induced by absorption of photons in the UV and EUV range and occur in the attosecond time scale. In particular, electron transfer along a molecular chain or between molecules is a central issue in many fundamental chemical and biological processes, and can be considered as a prototypical chemical reaction. ${ }^{414}$

The investigation of electron transfer and the development of experimental techniques to control it is a very active research field in physical chemistry. Electron transfer can be very slow, as e.g. in leakages across junctions in semiconductor devices, which are designed to take months, ${ }^{414}$ or very fast, as in electron transfer processes induced by photoexcitation, which can be more rapid than molecular vibrational periods. For example, as we will discuss below, the electron delocalization time in the conduction band of the deoxyribonucleic acid (DNA) occurs in about 740 as. ${ }^{415}$ Theoretical studies have shown that very efficient charge transfer can be driven by purely electronic effects, ${ }^{34,416}$ which precede any rearrangement of the nuclear skeleton and can evolve on a temporal scale ranging from few femtoseconds down to tens of attoseconds. ${ }^{34-37,253}$ This ultrafast charge dynamics has been referred to as charge migration.

In this section we will illustrate how the progress made in the study of electron dynamics in diatomic and small polyatomic molecules presented in the previous section has been transferred to systems of biological relevance. Experimental and theoretical investigations have run in parallel, focusing on partial aspects of the problem and on different observables. Due to the complexity of these systems, a complete description of electron dynamics is still to come, but the knowledge acquired during the last few years has already permitted to 
answer a number of crucial questions to achieve this goal. We will first review the existing experimental efforts and then theoretical predictions, which, although incomplete, are rather abundant and have covered a large range of aspects and systems.

\subsection{Experimental findings}

Pioneering experiments by Schlag and coworkers ${ }^{417-419}$ on polypeptide molecules, performed well before attosecond light sources were available, have inspired researchers in this field for several years. These molecules are composed by sub-units such as aminoacids, nucleotides, side chains and aromatic chromophores, linked by $\sigma$-bonds. Measurements were performed on peptides of natural aminoacids of the type $(X)_{n}-Y(n=1,2,3)$ in the gas phase, where $Y$ is an aromatic aminoacid (tryptophan, tyrosine or phenylalanine) located at the C-terminal end of the peptide and $X$ is a non-aromatic aminoacid located at the N-terminus. Peptides with a single aromatic aminoacid were used to generate local ionization at the chromophore site by resonant two-photon UV excitation ${ }^{417}$. After an additional one-photon UV excitation of the peptide cation, fragment mass spectra were measured. For example, in the mass spectrum of $(\mathrm{Leu})_{n}$-Trp $(n=1-3)$, where Leu is leucine and Trp is tryptophan, a fragment ion of mass 86 Da was predominant in the spectrum ${ }^{417}$. Such a fragment corresponds to a positively charged N-terminal fragment. Therefore, although a positive charge is originally generated in the chromophoric side chain of the C-terminal aminoacid, the charge is finally found at the N-terminal fragment ion. The same behavior has been measured in (Ala $)_{n^{-}}$ Tyr $(n=2,3)$, where Ala is alanine and Tyr is tyrosine, (Leu) $)_{n}$ Tyr, Ala-Ala-Tyr-Ala-Ala and other peptides ${ }^{418}$. Weinkauf et al. interpreted these experimental results in terms of a very efficient charge-transfer process that can occur over distances exceeding $10 \sigma$-bonds. The fact that the same results were obtained with molecules of very different sizes suggests that the mechanism responsible for this charge-transfer process is likely related to purely electronic effects rather than to nuclear motion. The temporal resolution of the first set of the measurements performed by Weinkauf and coworkers was limited by the nanosecond 


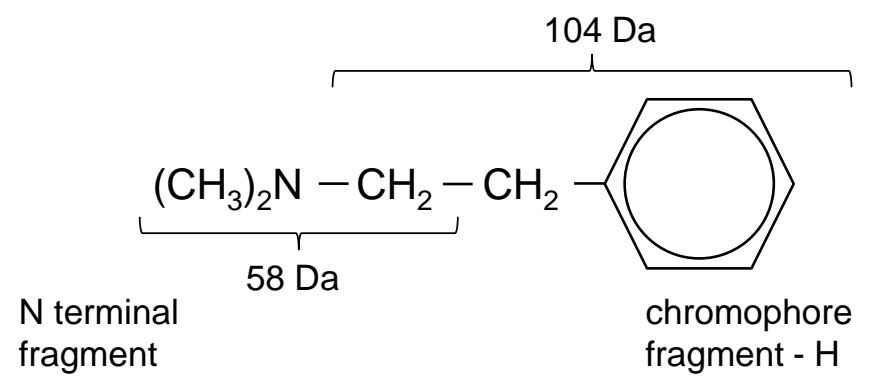

Figure 31: Schematic molecular structure of PENNA, with the indication of the N terminal and chromophore fragments.

laser pulse lengths.

In 2005, femtosecond dynamics following ionization of 2-phenylethyl- $N, N$-dimethylamine (PENNA) was reported. ${ }^{420}$ PENNA contains two ionization centers, a phenyl group and an amine group, separated by a $-\mathrm{CH}_{2}-\mathrm{CH}_{2}$ - spacer $\left(\mathrm{C}_{1}-\mathrm{C}_{2}\right.$ bond $)$, as shown in Fig. 31 . The molecule can be locally ionized at the phenyl site by resonant two-photon ionization (R2PI), as demonstrated by Cheng and coworkers. ${ }^{421}$ Femtosecond pump-probe experiments were performed by using 200-fs pump pulses with wavelength tunable between 230 and $280 \mathrm{~nm}$ and 120-fs probe pulses tunable in the range between 460 and $560 \mathrm{~nm} .{ }^{420}$ Figure $32(\mathrm{~A})$ shows the sum of the parent and the $\mathrm{N}$ fragment ion yields, i.e., the sum of intensities for the fragments with masses 149 and 58 Da, respectively, as a function of the pump-probe delay. Figure 32(B) shows a similar plot for the phenylethylene fragment ion (C fragment with a mass of $104 \mathrm{Da}$ ). These experimental results strongly support the conclusion that, after ionization at the phenyl chromophore, the molecule undergoes a charge transfer process towards the amine site with subsequent fragmentation leading to the immonium cation. Indeed, if the state corresponding to having the charge on the phenyl group had an infinite lifetime, $\tau$, the temporal evolution of the C-ion yield would exhibit a rise at $t=0$, similar to that of the pump-probe cross-correlation function, followed by a plateau at positive delays. In contrast, for finite lifetimes, the $\mathrm{C}$-ion yield would exhibit the same rise at $t=0$, but this time it would be followed by a decay of the ion signal at longer delays. Therefore, the decay 


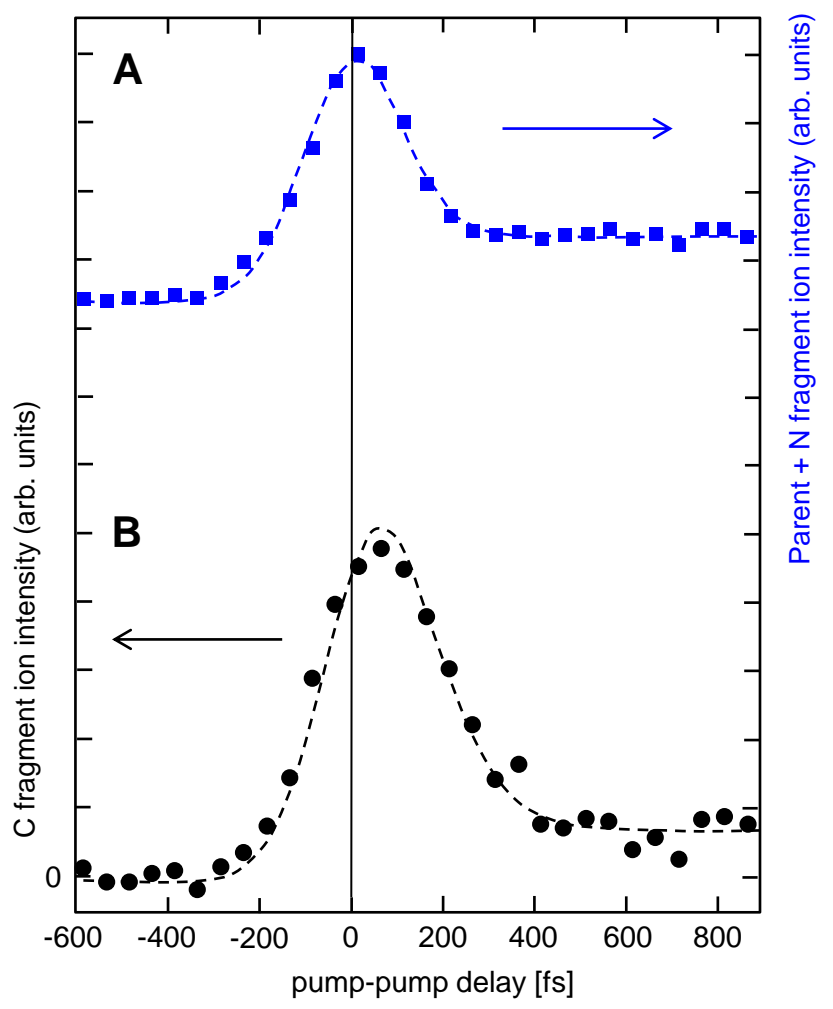

Figure 32: (A) Temporal evolution of the sum of the parent and the $\mathrm{N}$ fragment ions as a function of the pump-probe temporal delay (filled squares: experimental data; dashed curve: best fitting curve). (B) Temporal evolution of the phenylethylene fragment ion (C fragment: mass $104 \mathrm{Da}$ ) as a function of the pump-probe temporal delay (filled circles: experimental data; dashed curve: best fitting curve). Adapted from ref. [420] with permission. Copyright 2005 American Chemical Society.

in the ion yield that can be seen in Fig. 32(B) suggests that the initial state created by R2PI has a finite lifetime and that charge transfer occurs in $~ 80 \pm 28$ fs. Since this time constant is shorter than the duration of the pump and probe pulses, one can reasonable assume that the measured value is an upper limit for the duration of the charge transfer process between the amine group and the phenyl chromophore. For sure, such a rather large value cannot be the result of purely electronic dynamics.

Femtosecond dynamics initiated by femtosecond EUV pulses produced by HHG has also been recently reported for various polycyclic aromatic hydrocarbons (PAHs): naphthalene, anthracene, pyrene and tetracene. ${ }^{422}$ It was found that the excited molecule undergoes a 
relaxation process evolving on the timescale of a few tens of femtoseconds, which involves an interplay between the electronic and the vibrational degrees of freedom. ${ }^{358}$

By using shorter pulses, pump-probe measurements have been reported on the aromatic aminoacid phenylalanine (which presents structural similarities to PENNA) by Belshaw and coworkers. ${ }^{423}$ Also the radical cation of phenylalanine presents two charge-acceptor sites with approximately the same binding energy located on the phenyl and amine groups, ${ }^{424}$ separated by two singly bonded carbons (as shown in Fig. 33). Phenylalanine is an $\alpha$-amino acid, consisting in a central carbon atom ( $\alpha$ carbon), linked to an amine $\left(-\mathrm{NH}_{2}\right)$ group, a carboxylic group (-COOH), a hydrogen atom and a side chain (-R). The side chain is specific for each amino acid and for phenylalanine is formed by a methylene $\left(-\mathrm{CH}_{2^{-}}\right)$group ( $\beta$ carbon) terminated by a phenyl ring.

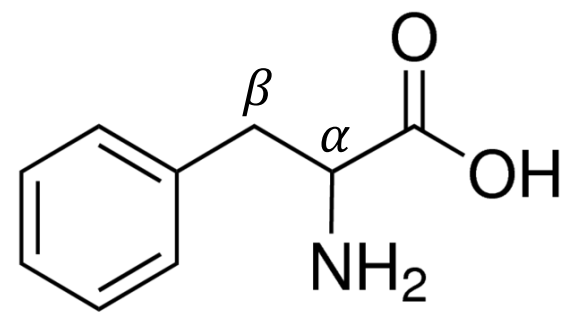

Figure 33: Schematic molecular structure of Phenylalanine.

In this case, EUV pump pulses with a duration of $1.5 \mathrm{fs}$ and a photon energy in the range between 16 and $40 \mathrm{eV}$ were used, followed by time-delayed controlled 6 -fs near-IR probe pulses, with photon spectrum ranging from 500 to $950 \mathrm{~nm}$. The parent and fragment ions produced by this combination of pulses were then detected by using a linear time-offlight device for mass analysis. Figure 34 shows a typical mass spectrum produced by EUV photoionization. The main contributions correspond to the parent ion $\mathrm{M}^{+}$(mass/charge, $m / q=165)$, the immonium ion, indicated as $\mathrm{Im}^{+}$in Fig. 34, resulting from the loss of the carboxyl group $(m / q=120)$ and fragments resulting from the breakage of the $C_{\alpha}-C_{\beta}$ bond with charge residing on the amine or phenyl groups $(\mathrm{R}, m / q=91, \mathrm{R}+\mathrm{H}, m / q=92)$. A small peak with a mass to charge ratio $m / q=60$ is visible, which corresponds to the doubly 
charged immonium ion. The EUV pulse is capable of ionizing all valence and some inner shell orbitals, resulting in a wide range of fragment ions. It was observed that using only the VIS/NIR pulses to ionize the molecule produced predominantly parent ions in the mass spectrum.

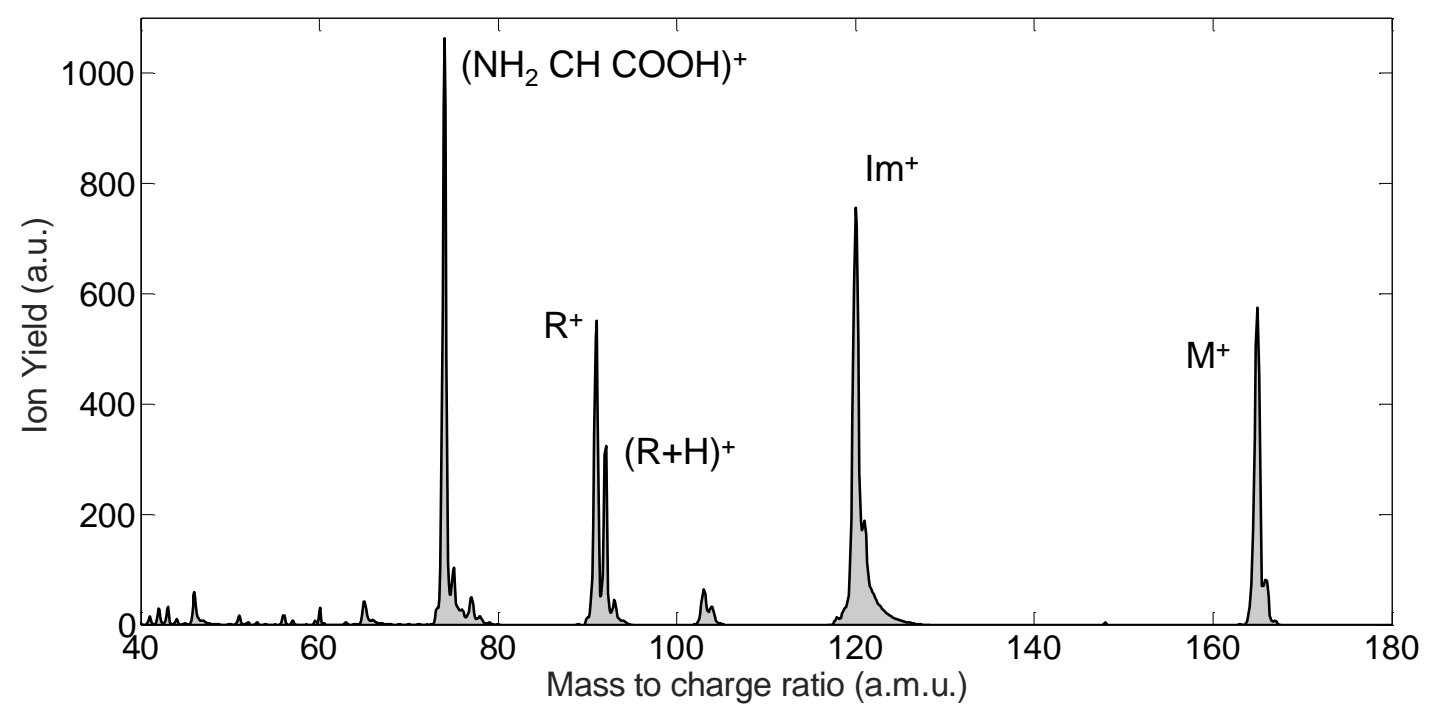

Figure 34: Mass spectrum from ionization of phenylalanine by an EUV pulse. M stands for the parent ion, $\mathrm{Im}^{+}$for the immonium ion and $\mathrm{R}$ for the side chain group.

The temporal evolution of the ion yields was measured as a function of pump-probe delay for all fragments. For some of the singly-charged fragments $(m / q=65,77,91,103)$, an increase for positive delays with a time constant of $80 \pm 20$ fs was observed. This temporal evolution has been attributed to an internal conversion process into the $\pi_{1}$ state of the phenyl radical cation following initial ionization of a different orbital by the pump pulse. A preliminary indication of ultrafast charge dynamics occurring in phenylalanine was obtained from the measurement of a fast decay in the doubly charged immonium ion $(\mathrm{m} / q=60)$ yield. The corresponding experimental data are shown in Fig. 35. A fit of these data to the function $F(t)$ that results from the convolution of

$$
R(t)=A\left(e^{-t / \tau_{1}}-e^{-t / \tau_{2}}\right),
$$


to a Gaussian function of 4 -fs full-width at half maximum (FWHM) led to $\tau_{1}=10 \pm 2$ fs and $\tau_{2}=25 \pm 2$ fs. Subsequent measurements performed in tryptophan and tyrosine evidenced that almost the same temporal evolution of the doubly-charged immonium ion is observed in all the aromatic amino acids, as shown in Fig. $35\left(\tau_{2} \approx 23\right.$ fs for tryptophan and $\tau_{2} \approx 20$ fs for tyrosine).

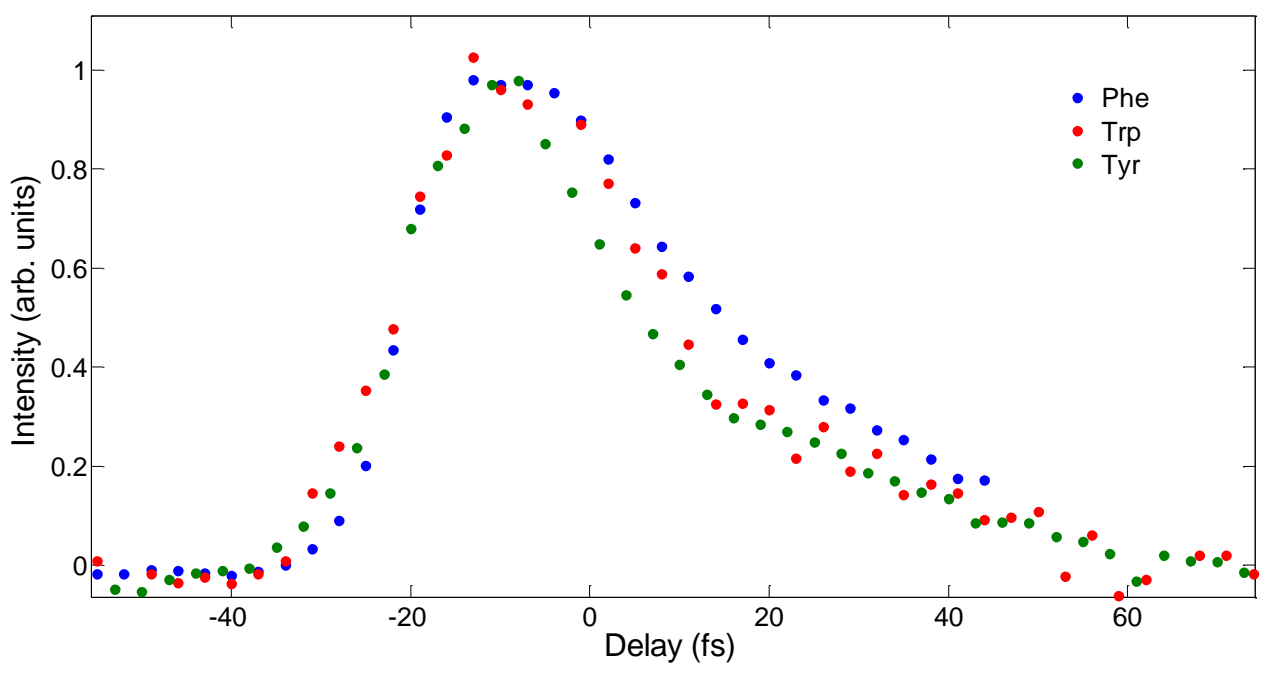

Figure 35: Temporal evolution of the doubly charged immonium ion yield as a function of the EUV pump NIR probe delay for phenylalanine (blue dotted line), tryptophan (red dotted line) and tyrosine (green dotted line).

In 2014 Calegari and coworkers reported on pump-probe measurements on phenylalanine performed with isolated attosecond pump pulses. ${ }^{25}$ Charge dynamics was initiated by isolated sub-300-as pulses, with photon energies in the spectral range between $17 \mathrm{eV}$ and $35 \mathrm{eV}$, and subsequently probed by 4-fs, waveform-controlled near infrared (NIR) pulses, with central wavelength of $720 \mathrm{~nm}$. A clean plume of isolated and neutral molecules of phenylalanine was generated by evaporation of the amino acid from a thin metallic foil heated by a CW diode laser. Isolated attosecond pulses, with energy in the nanojoule range, were produced by employing the ionization gating technique. The temporal duration of the EUV pulses $(290 \pm 20$ as) was measured by using the FROG CRAB technique. Charge migration was evidenced as a fast and periodic modulation of the dication yield. Figure 36(A) shows the 


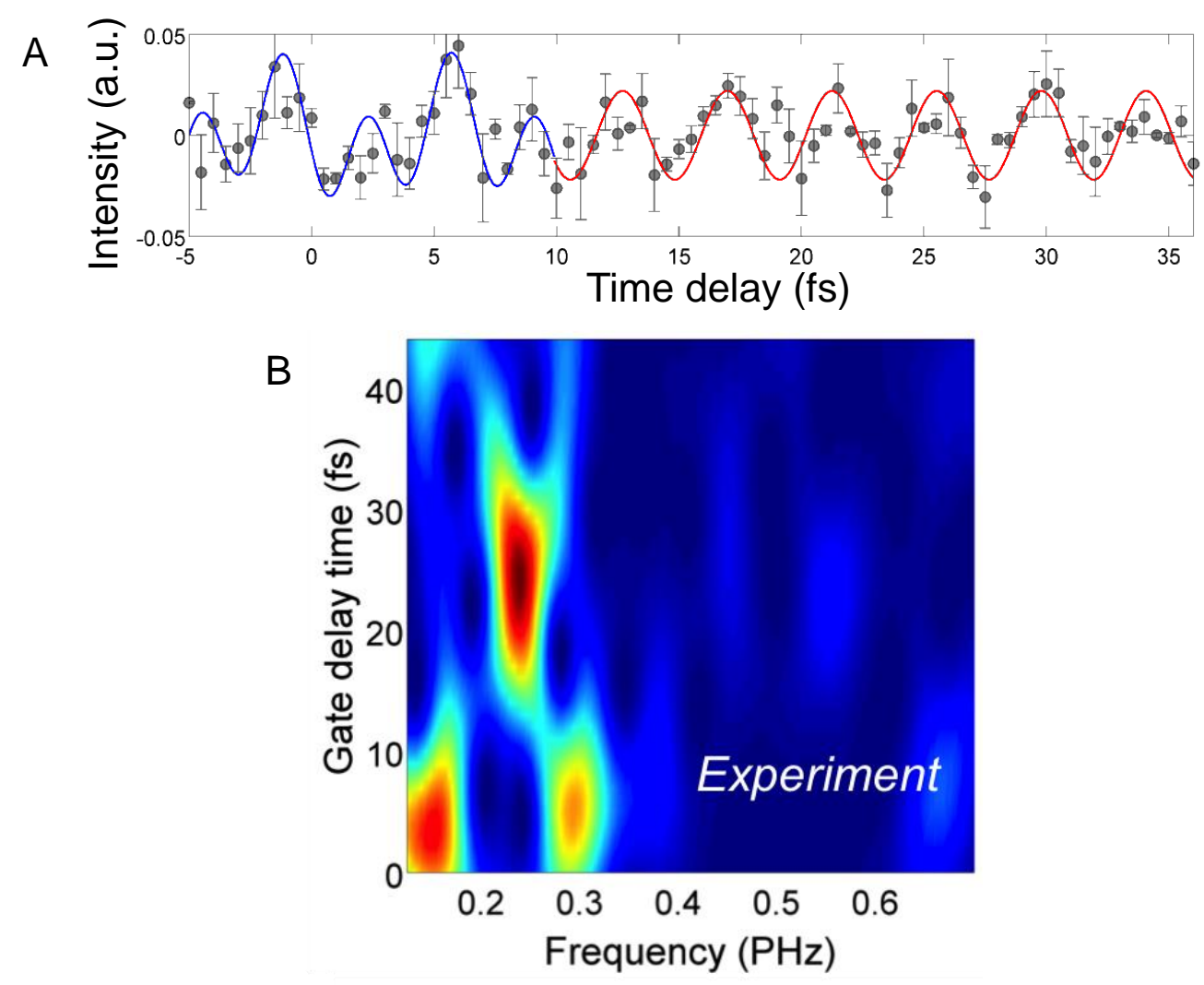

Figure 36: (A) Dots correspond to the difference between the experimental data and the fitting curve $\mathrm{F}(\mathrm{t})$. Error bars show the standard error of the results of four measurements. Blue curve is a fitting curve obtained as the sum of two sinusoidal functions, red curve is a single sinusoidal fitting curve, as described in the text. (B) Fourier sliding-window analysis of the experimental data. Adapted from ref. [ 25]. Copyright 2014 The American Association for the Advancement of Science (AAAS).

experimental data after subtraction of the fitting curve $F(t)$. Figure 36(B) displays the time-dependent Fourier analysis of the experimental results. At short pump-probe delays $(t<10 \mathrm{fs})$, two main frequency components are present, around $0.12 \mathrm{PHz}$ and $0.29 \mathrm{PHz}$. At longer pump-probe delays, (between $10 \mathrm{fs}$ and $36 \mathrm{fs}$ ) a strong and broad peak around $0.24 \mathrm{PHz}$ is visible in the Fourier spectrogram, corresponding to an oscillation period of 4.3 fs, shorter than the vibrational response of the molecule. By using the theoretical method discussed in Sect. 4.2, the 4.3-fs oscillations have been mainly assigned to periodic charge migration from the amine functional group to the phenyl group and back to the amino acid, as will be discussed in more details in Sect. 6.2. Note that the highest vibrational frequency 
is $0.11 \mathrm{PHz}$, which corresponds to a period of $8.9 \mathrm{fs}$, associated with $\mathrm{X}-\mathrm{H}$ stretching modes, whereas skeleton vibrations are even slower, so that one can rule out that the observed 4-fs oscillations are due to vibrational motion. In any case, the influence of the nuclear motion cannot be completely excluded, because, for example, stretching of the order of a few picometers of carbon bonds can occur in a few femtoseconds, and this could modify the charge dynamics. ${ }^{358,425}$

A completely different experimental technique, namely core-hole spectroscopy, was used by Ikeura-Sekiguchi and Sekiguchi to investigate electron delocalization in the conduction band through the phosphate backbone of genomic DNA. ${ }^{415}$ The goal was to understand electron transfer properties in DNA. Both periodic and aperiodic DNA backbone types were used in order to investigate the formation of extended Bloch-type orbitals along the backbone. Experiments were performed at beam line BL-27A of the Photon Factory, High Energy Accelerator Research Organization (KEK-PF) in Tsukuba, with energy resolution of 0.9 eV around the P K-edge using Resonant Auger Spectroscopy (RAS) and X-ray absorption Spectroscopy (XAS). XAS spectra were measured by using X-ray energies between 2150 and $2160 \mathrm{eV}$ in order to excite the $1 s$ core electron of phosphorus atoms in the backbone of the molecule to an empty conduction band of $t_{2}^{*}$ orbitals in phosphate groups. A typical $\mathrm{P}$ $K L_{2,3} L_{2,3}$ RAS of dry DNA at the $t_{2}^{*}$ transition $(2152.9 \mathrm{eV})$ is shown in Fig. 37A, where spectator and normal Auger peaks are clearly visible. Figure 37B shows the integrated intensities of spectator and normal Auger components near the $P K$ edge as a function of the excitation energy for dry and wet DNA and for the DNA oligomer, PD-oligo (used as an example of aperiodic backbone). As discussed in Sect. 3.3.1, the electron delocalization time, $\tau_{T}$, can be obtained by measuring the ratio between the intensities of spectator, $I_{2 h 1 e}$, and normal, $I_{2 h}$, Auger components: $\tau_{T}=\tau\left(I_{2 h 1 e} / I_{2 h}\right)$, where $\tau$ is the P $1 s$ core-hole lifetime, $\tau=1.25$ fs. ${ }^{426}$ The evolution of the electron delocalization time as a function of the excitation energy is shown in Fig. 37C. For wet DNA, the measured delocalization time is $740 \pm 30$ as at the excitation energy of $2153.7 \mathrm{eV}$, corresponding to a peak in the $2 h$ yield spectrum. The 

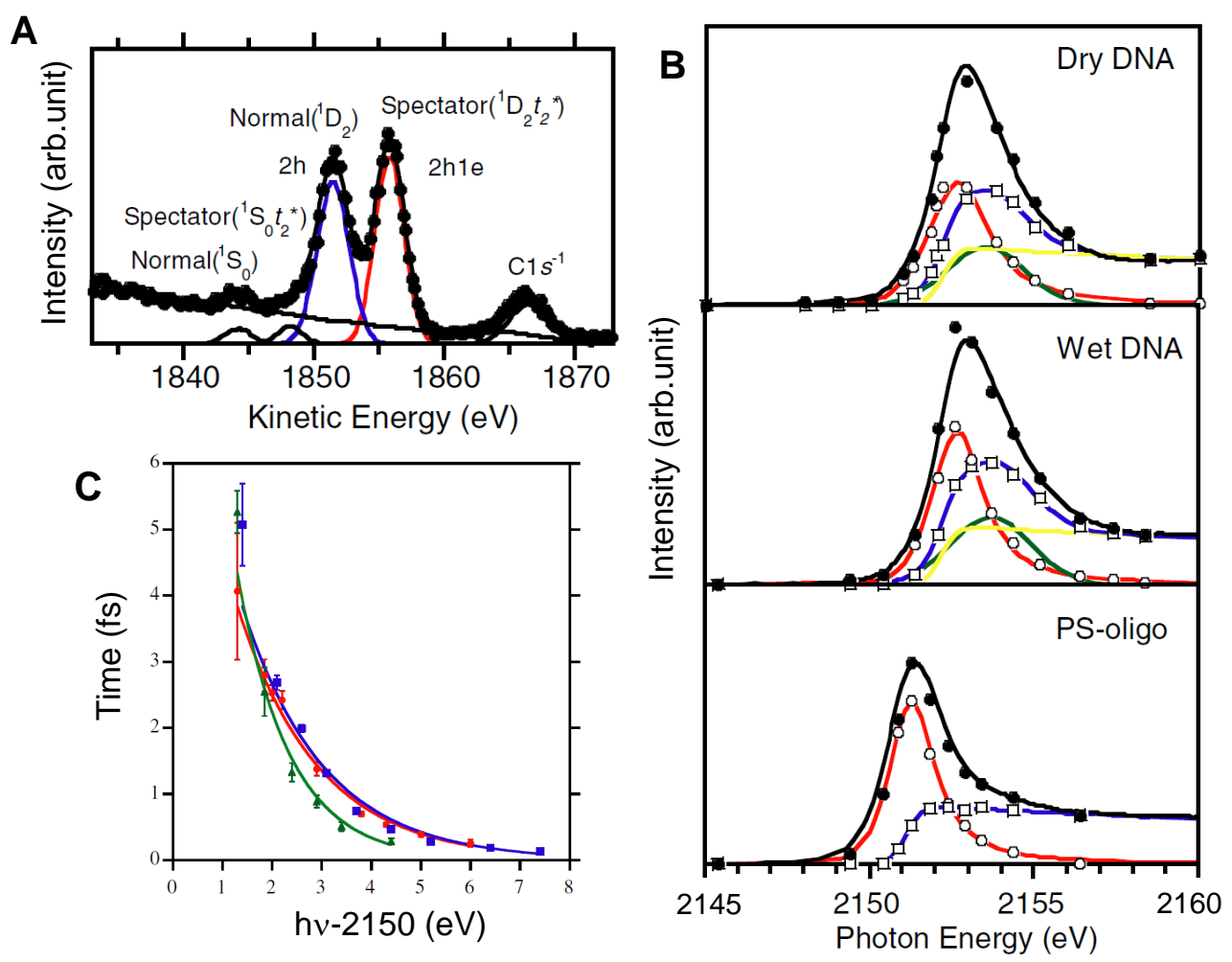

Figure 37: (A) Typical P $K L_{2,3} L_{2,3}$ RAS of dry DNA at the $t_{2}^{*}$ transition $(2152.9 \mathrm{eV})$. Dots: experimental data; solid lines: least-squares fit. Blue line represents the normal Auger component, while red line represents the spectator Auger component. (B) Integrated intensities of spectator (open circles) and normal (open squares) Auger components near the $P K$ edge for dry and wet DNA and for the PS-oligo. Results of curve fitting for $2 h 1 e$ and $2 h$ spectra are shown as red and blue lines, respectively. (C) Evolution of the electron delocalization time as a function of the excitation energy, for dry (circles) and wet (squares) DNA. Green triangles correspond to the ionization time for PS-oligo. The solid lines are exponential fitting curves. Adapted from Ref. [ 415] with permission. Copyright 2007 American Physical Society.

same experimental technique has been used to measure the femto- and attosecond electron dynamics in the 5'-Guanosin monophosphate (GMP) nucleotide. ${ }^{427}$ It was found that the electron delocalization time of the phosphate groups in GMP is faster than in DNA.

\subsection{Theoretical predictions}

Motivated by the experimental results of Schlag and coworkers, theoretical studies of charge migration were initiated by Zobeley and Cederbaum. ${ }^{34}$ Under the assumption of the "sud- 
den approximation" described in Sect. 4.2.1, they analyzed a number of systems, drawing important general conclusions as to the time scale and universality of the phenomenon, and on the connection between behavior of charge migration and the nature of the ionization spectrum. Similar approaches were also used by Remacle and Levine. ${ }^{35}$ Basically the ansatz is the creation of an electron wave packet corresponding to a removal of one electron from a frozen ground state orbital. The wave packet so formed is freely propagated in the basis of cation eigenstates at fixed nuclear geometry. The latter states, and the corresponding Dyson amplitudes, were obtained as eigenvectors of an effective hamiltonian matrix, the ADC(3) Green's Function approach in the work of Cederbaum and collaborators. Although free propagation is in principle trivial, the large dimensionality of the matrix is dealt with a Lanczos time propagating approach for the TDSE, discretizing the time steps, and diagonalizing the hamiltonian at each step in a small Krylov space generated by the current vector. ${ }^{267}$ Actually the term charge migration has been defined by Cederbaum et al. specifically referring to electronic motion in the fixed nuclei approximation, i.e. before any slower nuclear motion sets in. Besides studying the hole density propagation, Kuleff and Cederbaum ${ }^{428}$ realized that a time dependent dipole will be induced in the cation, and studied the radiation generated by this dipole, in two different regimes. In the first, a pulse creating a superposition of two outermost orbitals (HOMO and HOMO-1 in the MePENNA molecule) generate an IR pulse of frequency characteristic of the energy difference between the two cationic states, and would then constitute a characteristic signature of charge migration. In the second, ultrafast ionization of a single HF MO by a very broad pulse will induce a much stronger emission in the VUV, spanning a large energy range. Despite the important knowledge gained, such framework is very idealized with respect to possible attosecond pulse experiments, and recent research has focussed on analyzing and removing several approximations.

First is a realistic description of wave packet preparation, i.e. of the system after the pump pulse. This must include the effect of the electromagnetic field, either perturbatively or via TDSE, and the description of the continuum electron. A high energy and broad pulse 
will produce a manifold of ionic states according to transition probabilities, and a coherent superposition will be produced only among states lying within the pulse bandwidth. Related questions are decoherence of the cationic states from the emitted photoelectrons, and the dependence of the wave packet on the energy and shape of the pulse, which may offer possibilities of controlling the charge migration process. A critical issue is the influence of nuclear motion, both its interplay with the motion of the electronic wavepacket and the spread of nuclear configurations associated with the quantum probability distribution of nuclear positions, i.e. the vibrational distribution in the ground state. A final issue is the probing of the wave packet evolution. Typically it has been followed theoretically by mapping the hole density, ${ }^{34}$ either plotting it directly, or in terms of its natural orbitals and occupation numbers, ${ }^{267}$ or via Mulliken populations. ${ }^{429}$ It would be hard to directly observe the hole density experimentally. The usual observable is molecular dissociation, via time resolved mass spectrometric analysis, possibly after a second pulse to produce dissociation. This is quite hard to describe theoretically, and the link has been discussed in terms of chemical intuition, considering charge flow around a selected functional group or assuming charge localization after decoherence of the electronic wave packet due to ensuing nuclear motion. A more direct connection could be envisaged via detection of time resolved photoabsorption or photoelectron spectra and angular distributions generated by a second pulse, possibly in the perturbative regime not to distort the wave packet evolution itself.

We start with the analysis of typical charge migration processes described within the SA, and conclude with recent progress exploring the issues above, noting the outstanding difficulty of a complete coherent picture, to be directly compared with experimental observables.

Cederbaum and coworkers have investigated the charge migration process in various electronic systems after localized and prompt ionization ${ }^{267,356}$ by calculating the temporal and spatial evolution of the hole density generated by the excitation. Here we will mention a few interesting applications, starting from the investigation of charge migration in $N$-methyl acetamide, ${ }^{36}$ which is often used as the simplest model in studies of the peptide bond. The 
molecule consists of a peptide bond and two methyl groups on each end of the molecule. At time $t=0$, immediately after sudden ionization of a particular molecular orbital (the $13 a^{\prime}$ orbital), the hole charge is mainly localized near the oxygen atom and around the acetyl-C atoms. After 4.2 fs the hole charge has migrated around the $N$-methyl group, on the opposite side of the molecule with respect to the initial charge distribution. The hole charge returns to the original position at $t=8.4 \mathrm{fs}$. This is an example of the hole-mixing effect, which in the SA model refers to a rotation of HF orbitals in the ionic states, in this case the $13 a^{\prime}$ and $14 a^{\prime}$ MOs. Indeed, considering only these two orbitals reproduces closely the results of the full calculation. Similar ultrashort charge migration processes have been found in other complex molecules. In a particular conformer of glycine $\left(\mathrm{NH}_{2}-\mathrm{CH}_{2}-\mathrm{COOH}\right)$, which is the simplest amino acid and it is often used as a relatively simple prototype for the investigation of peptides and proteins, the hole charge migrates from one side of the backbone to the other one in a few femtoseconds. ${ }^{267}$ Indeed, in a first set of numerical simulations an electron was removed from the $11 a^{\prime}$ HF orbital. At $t=0$ the hole is completely localized in the orbital $11 a^{\prime}$, therefore it is localized mainly on $\mathrm{CO}$ and $\mathrm{OH}$ group, as shown in Fig. 38(a). A substantial charge migration is seen already after $\sim 5 \mathrm{fs}$, when the hole charge is migrated towards the central part of the molecule, around the $\mathrm{C}-\mathrm{CH}_{2}$ bond. An additional motion towards the $\mathrm{CH}_{2}-\mathrm{NH}_{2}$ bond is completed after 8 fs. At this instant, the natural charge orbital overlaps by more than $91 \%$ with the molecular orbital $12 a^{\prime}$. Then, the hole starts to move back towards the initial configuration. On the other hand, if an electron is suddenly removed from the $14 a^{\prime} \mathrm{HF}$ orbital, which is spread over the entire molecule, with a major localization on the N-terminal moiety, the hole charge moves towards the $\mathrm{COOH}$ moiety in about 3.5 fs. In this situation more than $90 \%$ of the hole is transferred to the $13 a^{\prime}$ orbital. Then the process proceeds in the opposite direction so that the hole returns to the original position after $\sim 7$ fs. The dependence of charge migration on nuclear geometry was subsequently explored in ${ }^{430}$ considering three most important glycine conformers, GlyI, GlyII and GlyIII. A strong dependence of the pattern was observed, traced to the different orbital composition 


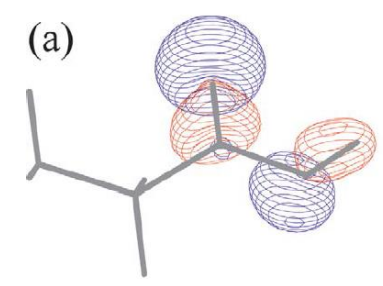

$t=0.0 \mathrm{fs}$

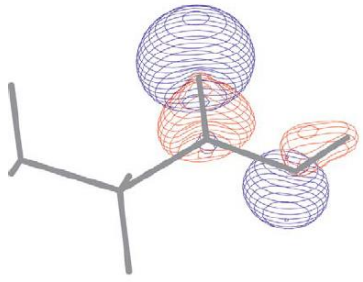

$t=1.7 \mathrm{fs}$

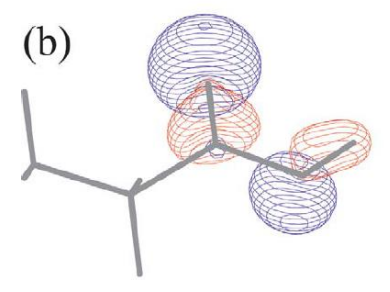

$t=3.4 \mathrm{fs}$

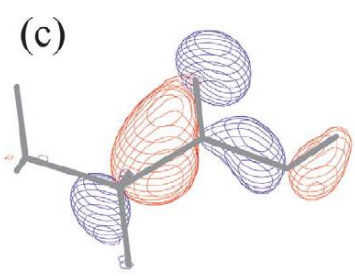

$t=5.1 \mathrm{fs}$

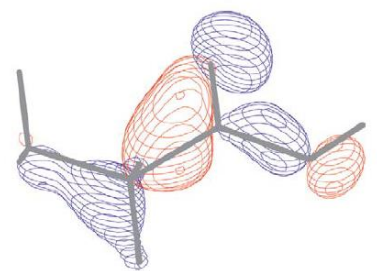

$t=6.8 \mathrm{fs}$

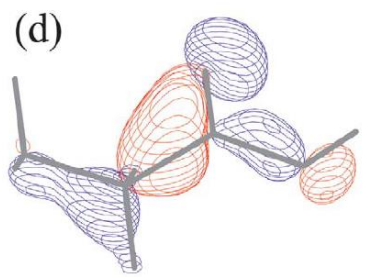

$t=8.4$ fs

Figure 38: Migration of hole density in glycine after ionization out of orbital 11a'. The molecule is oriented such that the $\mathrm{NH}_{2}$ group points left and the $\mathrm{OH}$ group right. (a) At $\mathrm{t}=0$ the hole density is identical to that associated with orbital 11a', which is localized mainly on the $\mathrm{CO}$ and $\mathrm{OH}$ groups. (b)-(c) A substantial part of the charge migrates to the $\mathrm{C}-\mathrm{C}$ bond. (d) At $\mathrm{t}=8.4$ fs the hole density is essentially that of the orbital $12 \mathrm{a}$ ' spread over almost the whole molecule, but having maximal density on the $\mathrm{C}-\mathrm{CH}_{2}$ and $\mathrm{CH}_{2}-\mathrm{NH}_{2}$ groups. Reproduced with permission from ref. [ 267]. Copyright 2005 AIP Publishing LLC.

in the conformers. Since symmetry is low $\left(C_{s}\right)$, it is not unexpected that canonical $\mathrm{HF}$ orbitals are quite sensitive to molecular conformation, and that reflects on the nature and time scales of charge migration. The time scale is determined by the energy separation of the cationic states involved, as is immediately apparent in the case of mixing of just two electronic states. The nature is reflected in the different Dyson amplitudes relative to electron removal from a particular canonical orbital in the different conformers. So, differently from GlyI and GlyIII, in GlyII ionization out of the $14 a^{\prime}$ orbital leaves the charge as well as the natural occupation number almost stationary, as it is mostly associated with a single cation 
state, with a small mixing of $2 \mathrm{~h} 1 \mathrm{e}$ satellites contributing to a tiny superimposed very fast oscillation. A dramatic slowing down of the oscillation period, in the range of $200 \mathrm{fs}$, is instead observed for ionization out of 11a' orbital in GlyIII, where again a pure hole mixing with 12 a' cation state is present, but the two states become almost degenerate, so that no apparent oscillation can be detected on a short time scale. This study gives a first hint of the importance of the distribution of nuclear geometries, which will be discussed later.

Electron dynamics initiated by prompt ionization has been calculated also in PENNA, ${ }^{358}$ in order to give a theoretical support to the femtosecond measurement reported in Ref. 420. Now the initial cationic state $\left|\Phi_{i}\right\rangle$ is prepared by removing an electron from the highest occupied molecular orbital (HOMO). In this case HOMO and HOMO-1 are localized on the phenyl ring (the $\pi$ orbital of benzene split by substitution), while HOMO-2 is localized on the the amino group. Because of correlation effects HOMO ionization becomes the second cationic state, and mixes with those derived from HOMO-1 and HOMO-2, all well separated by the rest of the valence shell, a case of a neat hole-mixing effect. This is purely due to correlation, as it cannot be described by a simple relaxation approach at the HF $\triangle \mathrm{SCF}$ level, as demonstrated in. ${ }^{431}$ At time $t=0$, the charge is localized on the phenyl ring; after 4 fs some part of the positive charge migrates to the N-terminal site. Then the charge returns mainly to the initial position and the process can start again. Here also the effect of nuclear geometry has been considered. It has been found that, upon increasing by 20 pm the length of the $\mathrm{C}_{1}-\mathrm{C}_{2}$ bond (mentioned in Sect. 6.1) almost the entire positive charge created by the initial ionization migrates from the phenyl ring to the N-terminal. This can be quite important, because first-principles molecular dynamics simulations in the cation shows a stretching of the $\mathrm{C}_{1}-\mathrm{C}_{2}$ bond of $5 \mathrm{pm}$ within the first $5 \mathrm{fs}$. Moreover, this is the bond which was observed to break in the experiments of Lehr and coworkers. ${ }^{420,421}$ On the basis of these considerations, a possible interpretation of the femtosecond measurement on PENNA is based on coupled electron and nuclear dynamics. After the generation of the positive charge on the phenyl ring, a portion of the charge starts to oscillate back and forth 
between this chromophore and the N-terminal. At the same time, the $\mathrm{C}_{1}-\mathrm{C}_{2}$ bond elongates as a result of nuclear dynamics thus leading to a more complete transfer of the positive charge. The oscillation process continues until the $\mathrm{C}_{1}-\mathrm{C}_{2}$ bond breaks and the charge is trapped at the energetically more favorable N-terminal fragment. The time constant of $\sim 80$ fs measured in Ref. 420 thus corresponds to the complete electron-nuclear dynamics which lead to the final trapping of the charge at the N-moiety.

The process of charge migration in complex biologically-relevant molecules has been theoretically investigated also by Remacle and Levine employing a simpler DFT approach. ${ }^{35}$ Also in this case, the numerical simulations show that charge migration in large molecules is possible on a sub-femtosecond time scale, driven by electron correlation. The tetrapeptides studied experimentally by Weinkauf and coworkers ${ }^{417,418}$ have been theoretically investigated, as the tetrapeptides Trp-Ala-Ala-Ala, Trp-Leu-Leu-Leu and Tyr-Ala-Ala-Ala, where an aromatic aminoacid is at a terminal position. It is assumed that prompt ionization ionizes a particular molecular orbital, for example the HOMO, of the neutral molecule. A hole is thus generated, which evolves in time since the HOMO of the neutral is not generally a stationary orbital of the cation. For example, in the case of Trp-Ala-Ala-Ala, the HOMO of the neutral is mainly localized on Trp, therefore the hole generated by prompt ionization is localized on Trp. The HOMO of the neutral is essentially a linear combination of the HOMO of the cation (molecular orbital 110) and the orbital 111 of the cation. Both these orbitals are localized mainly on the Trp and the $\mathrm{N}$ ends of the tetrapeptide. ${ }^{432}$ The hole generated on the Trp chromophore migrates to the $\mathrm{N}$ terminus of the peptide in 750 as, with a complete oscillation period of about $1.5 \mathrm{fs}$, which is in very good agreement with a simple estimation based on the energy separation, $\Delta E$, between the two molecular orbitals 110 and 111 of the cation: $\tau=h / \Delta E \approx 1.4$ fs. This oscillation continues until the motion of the nuclei leads to dephasing of the coherent superposition of states. Similar results were obtained in Trp-Leu-Leu-Leu. The situation is different in the case of sudden ionization of the HOMO-1 of the neutral, which is very similar to a molecular orbital (105) of the cation: in this case 
the hole remains stationary. ${ }^{432}$

\section{Excitation \\ energy $(\mathrm{eV})$}
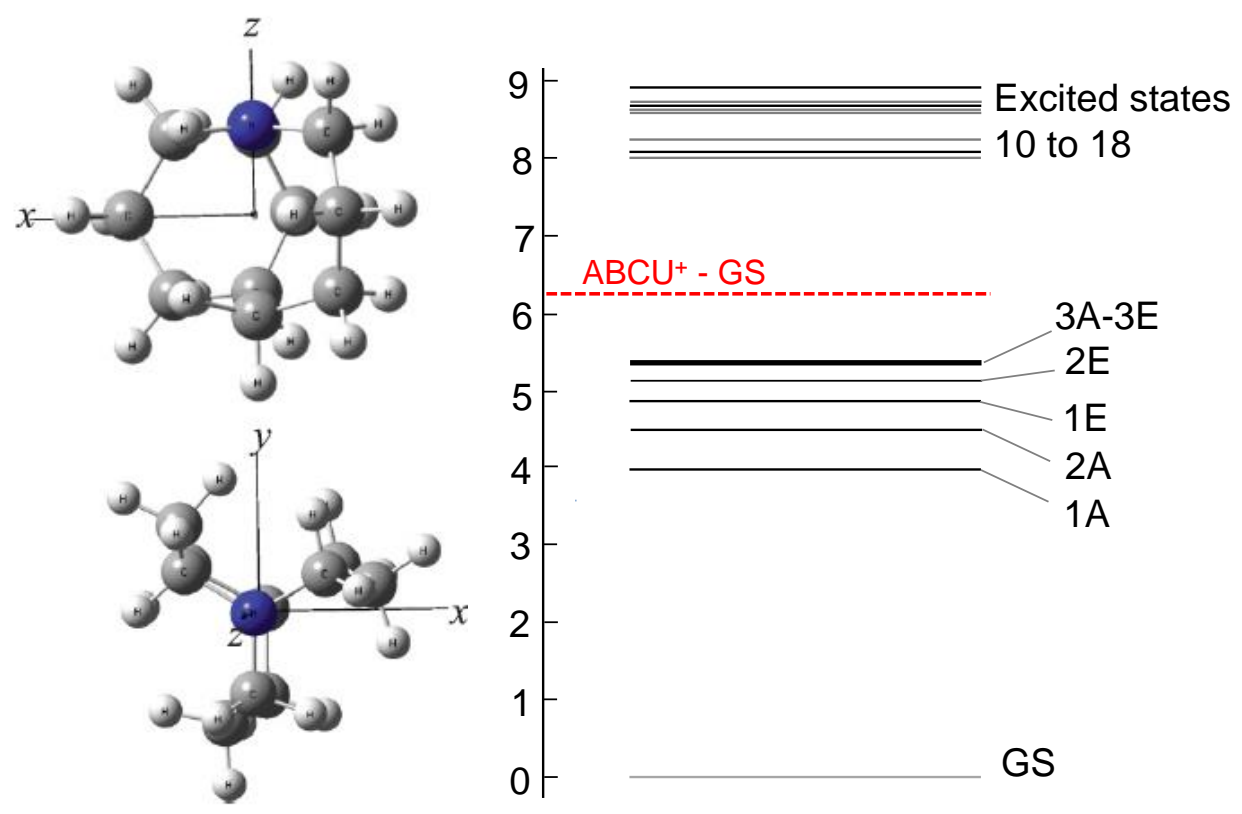

Figure 39: Molecular structure of the caged polyatomic molecule, 1azobicyclo[3.3.3] undecane $\left(\mathrm{C}_{10} \mathrm{H}_{19} \mathrm{~N}\right)$ (ABCU). The energy level diagram of the electronic states of $\mathrm{ABCU}$ was computed at the equilibrium geometry of the ground state(GS) at the CAS-SCF average level. In the calculations the pump pulse was optimized in order to create a coherent superposition of three states: the ground state and two low excited states, the states 2A and 1E. Reproduced with permission from ref. [ 293]. Copyright 2012 American Physical Society.

The role of proper treatment of correlation has been emphasized by Kuleff and Dreuw, ${ }^{431}$ who have analyzed the shortcomings of pure single particle approaches, which describe only relaxation via the $\triangle \mathrm{SCF}$ approach, as well as specific inadequacies of DFT treatments due to the poor description of the self-energy in currently used $V_{X C}$ potentials. Actually, as detailed in section 4 , the basic theoretical ingredients for the correct description of the final electronic wave packet are accurate cation energies and corresponding Dyson orbitals for the computation of transition dipole moments. In the outer valence, where only a few cation electronic states are accessible, both can be obtained by a variety of approaches, and do not appear very critical, when wave packet amplitudes are obtained via correct transition 
dipole moments. The extreme sensitivity to details of electron correlation may be a specific characteristics of the SA ansatz, where the only coupling responsible for charge migration is due to electron correlation.

A close-coupling approach along the lines of Sect. 4.1.2 was developed by Mignolet and coworkers in a series of papers. A first paper ${ }^{293}$ concentrates on the possibility of employing time resolved molecular frame photoelectron angular distribution (MFPADS) ${ }^{433-435}$ as a probe of the dynamics of excited states in the neutral induced by an attosecond pulse. The pump was described by solving TDSE in a small manifold of electronic ground and excited states. Ionization was treated perturbatively employing OPW for the continuum. Simulations were reported in the case of the four-electron diatomic molecule $\mathrm{LiH}$ and of a caged polyatomic molecule, 1-azobicyclo[3.3.3] undecane $\left(\mathrm{C}_{10} \mathrm{H}_{19} \mathrm{~N}\right)(\mathrm{ABCU})$. Figure 39 shows the molecular structure and the energy level diagram calculated for ABCU. In the calculations the pump pulse was optimized in order to create a coherent superposition of three states: the ground state and two low excited states, the states $2 \mathrm{~A}$ and $1 \mathrm{E}$ shown if Fig. 39. Fast beatings of the electronic density were calculated, which were generated by a superposition of the beatings between the ground state and the $2 \mathrm{~A}$ state and between the ground state and the $1 \mathrm{E}$ state, with a period of the order of $1 \mathrm{fs}$, related to the energy separation between the ground state and the low excited states. An additional beating with a slower period of 11.28 fs was obtained, related to the energy difference between the two excited states $\left(\Delta E_{A-E}=0.36 \mathrm{eV}\right)$. Moreover, it was clearly demonstrated that the MFPADS reflect the spatial localization of the coherent superposition of states. In 2013 Kuś et al. applied the same theoretical approach to the investigation of charge migration in peptides. ${ }^{436}$ It was found that in the investigated peptides, whose molecular chains extend over $15 \AA$, the most efficient charge migration mechanism is sequential, since it involves transitions between neighbor and next-neighbor aminoacid subunits. This model has been extended in ${ }^{295}$ by including both neutral excited states and the discretized continuum in a partitioning approach to the TDSE, to investigate the use of an EUV attosecond pulse train 
as a probe of ultrafast dynamics in LiH initiated by an IR pump. By tuning the time interval of the pulses within the train to a beating frequency of the WP it was possible to follow the changing spatial localization of the WP between the two ends of the molecule. Charge migration in the PENNA molecule was reinvestigated in, ${ }^{294}$ employing now close-coupling with a partition including, besides neutral excited and the singly ionized cation states, also doubly ionized cation states plus two continuum electrons, to describe photoionization of the cation by the probe pulse. Several approximations were employed, with the continuum basis consisting of orthogonalized continuum waves over a discrete energy and angular grid, for a total of 900 basis functions, coupled to 10 cation and 2 dication states, plus 30 neutral excited states. A DFT description was employed for the bound states, in good agreement with CASSCF results. Notably Dyson orbitals were found very close to canonical KohnSham orbitals, with same localization as previously described in. ${ }^{358}$ Two pulse sequences were considered: a UV pump (134 nm, $1.4 \mathrm{fs}$ ) followed by EUV probe (93 nm, $0.4 \mathrm{fs})$, and an IR pump (800 nm, $6 \mathrm{fs}$ ), EUV probe (93 nm, $1.4 \mathrm{fs})$. In all cases the pump essentially populates a coherent superposition of the three outermost cation states, and subsequent probe reaches the two lowest dication states. Evolution of the populations and the dipole moments showed the expected charge migration between the amino and the the phenyl groups. A signature of charge migration could be seen in the anisotropy of electron emission, which shows beatings related to the oscillations of the WP.

The first pump-probe measurements on the aromatic aminoacid phenylalanine performed by using isolated attosecond pulses, which we have mentioned in Sect. 6.1, motivated a lot of theoretical research, whose key aspects have been discussed in sect. 4. A realistic preparation of the electronic wave packet was possible, employing the same attosecond pulse as used in the experiment, and computing the resulting amplitudes by first order time dependent PT, which is completely adequate at the actual intensity. Accurate transition dipole moments to ionized states were computed via the static-exchange DFT approach presented in 4.1.4. Cation energies were computed via the accurate OVGF approach. ${ }^{340}$ Hole density matrix 
was evaluated assuming fast decoherence with the photoelectron. In order to understand the influence on the ultrafast electron dynamics of different radicals in the aminoacid, a systematic investigation has been performed not only in phenylalanine, but also in glycine, already investigated by Kuleff et al., ${ }^{267}$ and tryptophan. The first observation is that an attosecond excitation cannot remove electrons from a single molecular orbital, but from several orbitals, thus leading to the creation of a coherent superposition of ionic states. Ionization amplitudes were calculated for all open channels (15 in the case of glycine, 32 for phenylalanine and 39 for tryptophan); then the hole density was calculated and its free evolution on the molecular skeleton. Figure 40 shows snapshots of the relative variation of the hole density with respect
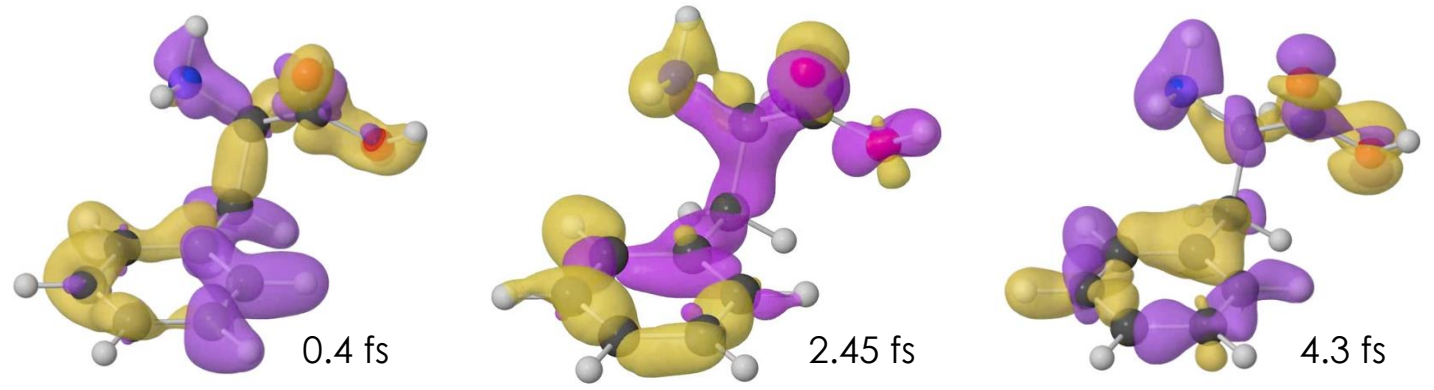

Figure 40: Snapshots of the relative variation of the hole density with respect to its timeaveraged value for the most abundant conformer of phenylalanine. Isosurfaces of the relative hole density are shown for cutoff values of $10^{-4}$ (yellow) and $-10^{-4}$ (purple) arbitrary units. Adapted from ref. [ 25]. Copyright 2014 The American Association for the Advancement of Science (AAAS).

to the time-averaged values for phenylalanine. In spite of the very delocalized nature of the hole density resulting from the broadband EUV excitation, a substantial redistribution of this density is observed on a sub-femtosecond temporal scale. The calculated hole dynamics cannot be associated with a simple migration from one side of the molecule to the other. However, despite the complexity of the charge configuration calculated in a realistic situation, the concept of charge migration is still valid. This is even more evident when the hole density is integrated around particular portions of the molecules and the corresponding Fourier power spectra are calculated. Particularly interesting is the charge dynamics around 
the amino group, since in the pump-probe experiment on phenylalanine, ${ }^{25}$ absorption of the probe pulse is more likely to occur from that part of the molecule due to the fact the HOMO orbital is significantly localized there. From the Fourier spectra it is possible to identify the states involved in predominant beating peaks, whose frequency is determined by the energy difference between two ionic states. For instance, one of the most intense beating in the amino group of phenylalanine occurs between the states with holes in the orbitals $41 a$ and the $44 a$ orbitals, both having nodal planes that contain the $\mathrm{C}-\mathrm{N}$ and the $\mathrm{C}=\mathrm{O}$ bonds with very similar orientations. ${ }^{254}$ By calculating the sliding-window Fourier transform of the temporal evolution of the hole density around the amino group as shown in Fig. 41, a clear

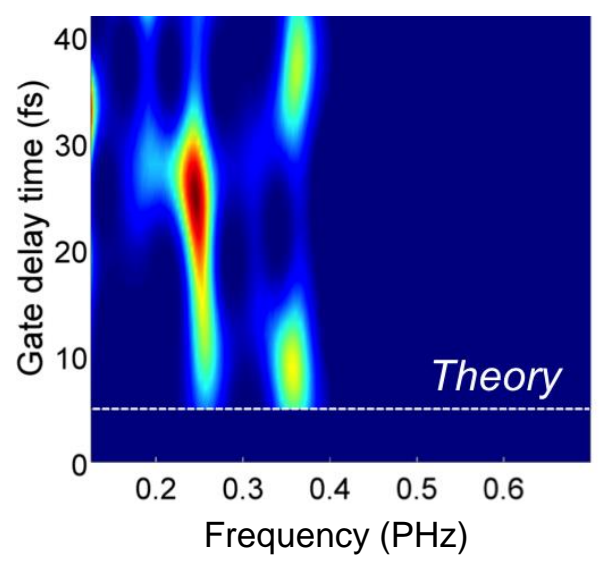

Figure 41: Fourier sliding-window analysis of the calculated temporal evolution of hole density integrated over the amine group for the most abundant conformer of phenylalanine. Adapted from ref. [ 25]. Copyright 2014 The American Association for the Advancement of Science (AAAS).

peak at $0.25 \mathrm{PHz}$ is visible, in very good agreement with the pump-probe measurement, which forms in about 15 fs and vanishes after about 35 fs. The temporal evolution of the main Fourier components is a consequence of the complex interplay among several beating processes initiated by the broadband excitation pulse. Despite the agreement with the experimental results, contributions related to nuclear dynamics, which are not included in the simulations, have to be taken into account for a detailed description of the charge dynamics.

A number of issues have been subsequently explored in glycine. ${ }^{380}$ The first is the role and time scale of coherences between the photoelectron and the remaining cation, which 
was totally neglected in the previous study, as in most other treatments. Computing time dependent one particle density matrices both for the $N$-electron system including the photoelectron, and the $(N-1)$-electron cation, as previously done, the evolution of the charge density after the pulse was compared. The results clearly show that the coherence of cation electronic states with the photoelectron is lost in less than $1 \mathrm{fs}$, even for photoelectrons of relatively low kinetic energies, as in the case of the pulse employed, so that neglect of the latter after the first $0.5-1$ fs is totally justified. Similar time scale for coherences was also found earlier in ${ }^{312}$ in the case of Xenon atom ionization. Second, the influence of nuclear motion induced by the non stationary wave packet has been explored by Ehrenfest dynamics within TDSE. Evolution of electron densities after sudden removal of one electron from the $14 \mathrm{a}^{\prime}$ or $4 \mathrm{a}^{\prime \prime}$ orbitals was investigated within the same approach, either keeping nuclei fixed, or allowing them to move. The evolution of the hole density was very close for about the first 8 fs, then started to deviate, becoming completely different after 25 fs. This result follows closely that one previously studied with the same approach in, ${ }^{252,429}$ and attributed to nonadiabatic transitions between different electronic states. Nonetheless, dominating frequencies in the Fourier transform of the hole densities around individual atoms confirmed the appearance of a limited number of peaks, and the overall pattern was not destroyed by nuclear motion, despite some slight changes in frequencies, or even the appearance/disapperance of a few peaks. A third issue is the influence of the energy and pulse shape on the specific WP evolution. Three different WP were considered, relative to current experimental pulses. It was found that significant differences in the Fourier frequencies were produced, despite the fact that all cationic states were populated by each pulse. It appears that pulses at relatively low photon energies may offer greater possibilities of control, since individual amplitudes are much more sensitive to the pulse shape close to threshold, where they are more structured, than in the high energy region. Last, a first attempt to describe pump-probe with two EUV pulses was performed, employing second order time dependent perturbation theory, which simplifies when the two pulses do not overlap in time, as in sequential double 
ionization. Employing the same set of orbitals, not a too severe approximation that can be easily lifted, the dication yield and its Fourier transform were considered as a function of time delay between the pulses, and found to reflect the main frequencies previously found for the densities around individual sites, although some details are lost. This shows that in this regime dication yield can be used to monitor the pump-induced dynamics without introducing additional distortion.

An interesting possibility for control of charge migration has been considered in, ${ }^{5}$ where a control pulse following the pump is applied to drive the charge to a selected localization, by tailoring the form of the wave packet. The methylated PENNA after ionization of HOMO or HOMO-1 is well described as a two level system, the following cation state being well separated in energy, so that a wave packet of these two cation states could be produced by a suitable pump pulse, without invoking the SA. The authors then devise an analytical expression for the pulse able to bring an arbitrary two state WP to a single state, either HOMO or HOMO-1, and demonstrate that effective charge localization can be achieved starting from an SA wave packet, even taking the whole cation manifold into account via the $\operatorname{ADC}(3)$ spectrum. It is interesting to note that even in molecules lacking any symmetry, the coherent superposition of two cationic states corresponding to ionization from two well-localized MO's can lead to a complete hole transfer between two ends of the molecule, as in the case just mentioned. This is more clean however in centrosymmetric molecules, when a superposition of gerade and ungerade orbitals obtained by combination of two identical moieties, gives back and forth oscillations of the hole density, like in polycyclic norbornadiene. ${ }^{268}$

The nuclear motion problem is a central issue in the charge migration context. It is expected to be a major source of decoherence, since energy differences between cation states, varying along the potential energy surfaces, alter the fundamental frequencies of the wave packet. The survival of charge migration to nuclear displacement in benzene has been considered in more detail in. ${ }^{376}$ An SA ionization of HOMO-2, $1 a_{2 u} \pi$ orbital gives a mixing of a primary cation state and a $2 \mathrm{~h} 1 \mathrm{e}$ satellite, involving a double hole in the HOMO and an 
excitation in the LUMO, which is quite distant in energy, $4.42 \mathrm{eV}$, corresponding to a period of 935 as. The relative charge oscillation corresponds to a breathing mode of the hole density. The influence of the 20 normal modes on the charge oscillation was investigated repeating the calculation for each normal coordinate in turn strongly displaced from the equilibrium value. Three different behaviors were observed: a) modes which cause a strong change of the ionization spectrum, with more states coupled to the $1 a_{2 u}$ ionization; b) modes which diminish the amplitude of satellite mixing; and c) modes which cause a minor variation of the spectrum. The first type destroys the coherence, the second tends to quench it, while the rest changes little with respect to the fixed nuclei behavior. However, by averaging the dynamics over all nuclear modes, computed at various displacements compatible with vibrational excitation in the ion, simulated by Boltzmann population at $3000 \mathrm{~K}$, a very weak damping of the fixed nuclei charge oscillation is observed, showing the robustness of this WP in benzene with respect to vibrational excitations. As hole density oscillates mostly around $\mathrm{H}$ atoms, and $\mathrm{H}-$ loss is a major decay channel in benzene, observation of $\mathrm{H}$ loss after nonlinear IR probe can provide an effective observable for this process.

The effect of nuclear motion on WP dynamics has been studied by Robb and collaborators in a series of papers. ${ }^{252,429}$ Basically starting from an arbitrary chosen electronic WP (often by the SA), Ehrenfest dynamics was employed to propagate the WP and the nuclear coordinates. Classical mechanics is employed for the nuclei, with forces computed as gradients of an average surface defined by the energy of the nonstationary electronic wavefunction. The advantage of the Ehrenfest model is to provide a uniform methodology that allows one to treat electronic propagation both with fixed nuclei and coupled with classical nuclear dynamics with the same formalism, so as to make a clear assessment of the importance of the latter. The main results are similar to those of the later work: ${ }^{380}$ mostly charge migration oscillations persist, at least on a short time scale of a few tens of fs, when nuclei are allowed to move, although details are changed due to changing of energy separation between cationic states. In the case of glycine and the Gly-Gly-NH-CH 3 cation the charge oscillations between 
the lone pairs associated with the carbonyl and amino groups remain almost unchanged after inclusion of nuclear motion even up to $40 \mathrm{fs}$ in glycine. ${ }^{252}$ Important dependence on molecular conformation was observed, as already discussed in Ref. 358. A more dramatic result was revealed in, ${ }^{268}$ were instead the effect of the spread of initial nuclear geometries, associated with ground state vibrations, was considered. A two electronic state analytical model, with a single nuclear coordinate, illustrates the origin of the effect. If the potential energy curves of the two electronic (cationic) states run parallel, the oscillation period is independent of the nuclear coordinate and no effect is observed after averaging over the initial state vibrational distribution. If however a gradient exists of the electronic energy difference, this is reflected in a range of energy differences and therefore oscillation periods, and the resulting averaging quickly destroys the coherence by dephasing, with a gaussian damping in time, and a characteristic lifetime inversely proportional to the gradient. This analysis is confirmed by explicit simulation of charge migration in paraxilene and polycyclic norbornadiene (PNL), after an assumed 50:50 or 60:40 superposition of the two lowest cationic states, corresponding to electron removal from the two outermost $\pi$ orbitals. With fixed nuclei at equilibrium geometry a neat charge migration oscillation is observed, and allowing nuclear motion in the cation via Ehrenfest dynamics does not alter significantly the oscillations although the period is progressively reduced. On the contrary if a Wigner distribution of nuclear coordinates is assumed to describe the quantum distribution of nuclear coordinates associated with vibrational motion in the ground state, the spread of oscillation periods causes a dramatics damping of the oscillations upon averaging, with a lifetime of a few fs in paraxilene and about $10 \mathrm{fs}$ in PLN. In a way, this is the analogous situation as that described for charge migration when ionization in the inner valence shell populates a multitude of closely spaced final electronic states, ${ }^{356}$ and reminds of the exponential decay of a discrete state coupled to a quasicontinuum. This aspect has been further explored in 2-phenyl-ethylamine and PENNA in, ${ }^{437}$ confirming that the dephasing due to nuclear spatial delocalization occurs on a much shorter scale than the effect of nuclear motion in the cations. Control of nuclear 
dynamics via a specific preparation of the initial electronic wave packet has been explored in a model two states description of ultrafast ionization in toluene. ${ }^{438}$

A fuller quantum mechanics description of coupled electron nuclear dynamics is only possible in smaller systems. Exploring wave packet evolution after ultrafast electronic excitation is also simpler, and offers in principle a neat possibility of detailed probing via time resolved photoelectron spectroscopy (TRPES), ${ }^{439,440}$ with a close match between theoretical description and experimental results. Several steps in this direction have been presented in a series of papers by Vibok and collaborators. ${ }^{441-443}$ Selective excitation of the $\mathrm{O}_{3}$ molecule to the B state (Hartley band) can be experimentally feasible with moderate intensity pulses. Evolution of the combined electronic-nuclear wavepacket, expressed in the close-coupling form

$$
\Psi_{t o t}(\mathbf{r}, \mathbf{R}, t)=\sum_{k} \Psi_{n u c}^{k}(\mathbf{R}, t) \psi_{e l}^{k}(\mathbf{r}, \mathbf{R})
$$

is solved for the nuclear wavepacket $\Psi_{n u c}^{k}$ employing the MCTDH approach. ${ }^{444-446}$ Evolution of the wavepacket shows charge oscillations between the two terminal oxygens corresponding to the two resonance forms in a valence bond description. Simulations with a 500 as EUV pulse at $95 \mathrm{eV}(1.5 \mathrm{eV}$ width) shows that this can be followed in principle by TRPES, and that signatures from ionization of the excited state can be disentangled from those of the ground state, and followed in time. ${ }^{447}$

\section{Towards attosecond molecular imaging}

The combination of attosecond time resolution with sub- $\AA$ spatial resolution provided by the HHG process (see section 2) led the strong-field community to explore, from the very beginning, the possibility of using high harmonic emission and related strong-field processes to image molecular structure and, why not, variations of this structure with time. In this section, we briefly describe progress made along this direction. Besides HHG, we will also discuss strong-field ionization techniques in which returning electrons that do not recombine 
(i.e., do not lead to high harmonic emission) are detected after rescattering by the parent molecular ion, thus carrying structural information on the latter.

\subsection{Tomographic imaging of molecular orbitals}

Since HHG is the result of the interference between the recolliding electron wave packet and the bound state wave function, from the HHG emission it is possible to extract information on the electronic structure of the target molecule. This information is contained in the amplitude and phase of the photo-recombination matrix elements, from which a tomographic reconstruction of the molecular orbitals can in principle be achieved. From the experimental point of view, molecular tomography is performed by measuring the harmonic spectra emitted by molecules aligned in space. Impulsive alignment ${ }^{448,449}$ is exploited to control the angle between the molecular axis and the polarization direction of the driving field. Harmonic spectra are acquired at a series of angles between the molecular axis and the recolliding electron. All the features related to the molecular structure and dynamics appear in the harmonic spectrum as modulations in the spectral shape. Spectral features such as local minima can be interpreted as electronic structure minima related to the nature of the highest occupied molecular orbital (HOMO) from which the electron is selectively ionized. ${ }^{450-452}$

Molecular tomography was first proposed and demonstrated in 2004 by Itatani et al., who achieved reconstruction of the HOMO of $\mathrm{N}_{2} \cdot{ }^{453}$ Figure 42 shows the comparison between (a) the tomographically reconstructed HOMO of $\mathrm{N}_{2}$ obtained from the harmonic spectra taken at 19 projection angles and (b) the shape of the same orbital obtained with an ab initio calculation. As can be seen from the cuts along the internuclear axis, the experimentally retrieved shape of the HOMO is in very good agreement with the calculated one. It is worth mentioning that, although a complete tomographic reconstruction requires both the amplitude and phase of the harmonic emission, in this pioneering experiment only the harmonic intensity was measured, and the harmonic phase was artificially introduced. A few years 

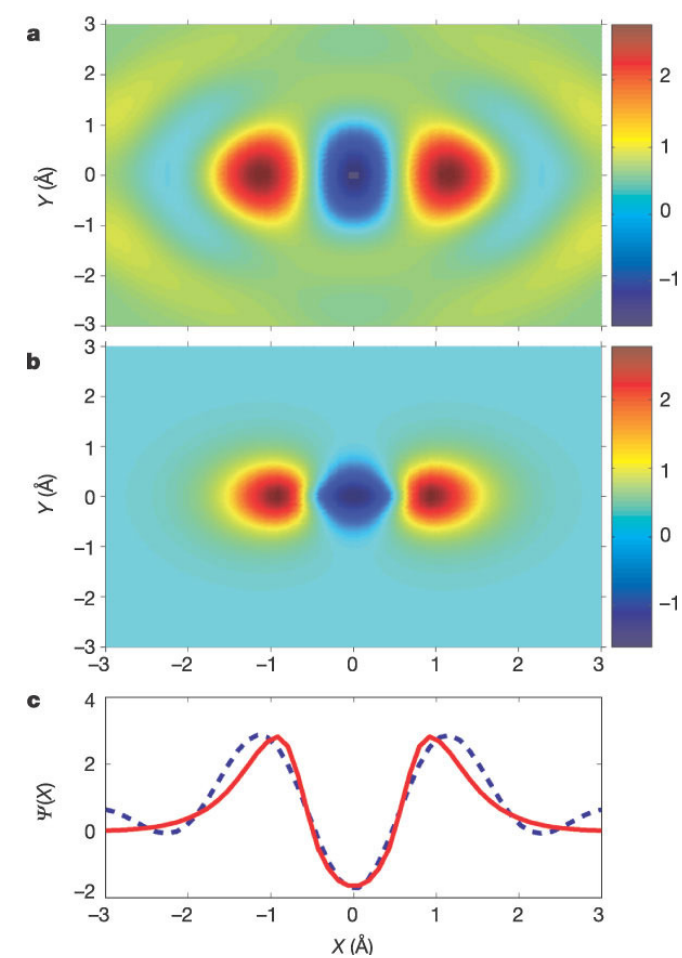

Figure 42: (a) Reconstructed HOMO of $\mathrm{N}_{2}$ obtained from a tomographic inversion of the high harmonic spectra. (b) HOMO of $\mathrm{N}_{2}$ obtained from an ab initio calculation. (c) Cuts along the internuclear axis for the reconstructed (dashed) and ab initio (solid) wave functions. From ref. 453 with permission. Copyright 2004 Macmillan Publishers Limited.

later, a more sophisticated approach based on the measurement of the harmonic phase with the RABBITT technique allowed for a precise reconstruction of the $\mathrm{HOMO}$ of $\mathrm{CO}_{2} \cdot{ }^{454}$ In 2011 a self-referencing approach and a generalization of the tomographic procedure, which exploits an all-optical technique, was employed for the tomographic reconstruction of $\mathrm{CO}_{2} \cdot{ }^{455}$ In this case, the amplitude and phase of the harmonic field emitted by aligned molecules was extracted by combining the measurement of the angular dependent harmonic spectra and a theoretical model that takes into account the Coulomb potential seen by the recolliding electron wavepacket in a first-order perturbation approach. The measured high harmonic spectra are shown in Fig. 43 (a). As can be seen, they exhibit a pronounced minimum at around harmonic 61 . Fig. 43 (b) shows the molecular orbital that was retrieved from these measurements. This reconstructed orbital reproduces very well all the features of the HOMO obtained from quantum chemistry calculations [Fig. 43 (c )]. From the reconstructed HOMO 

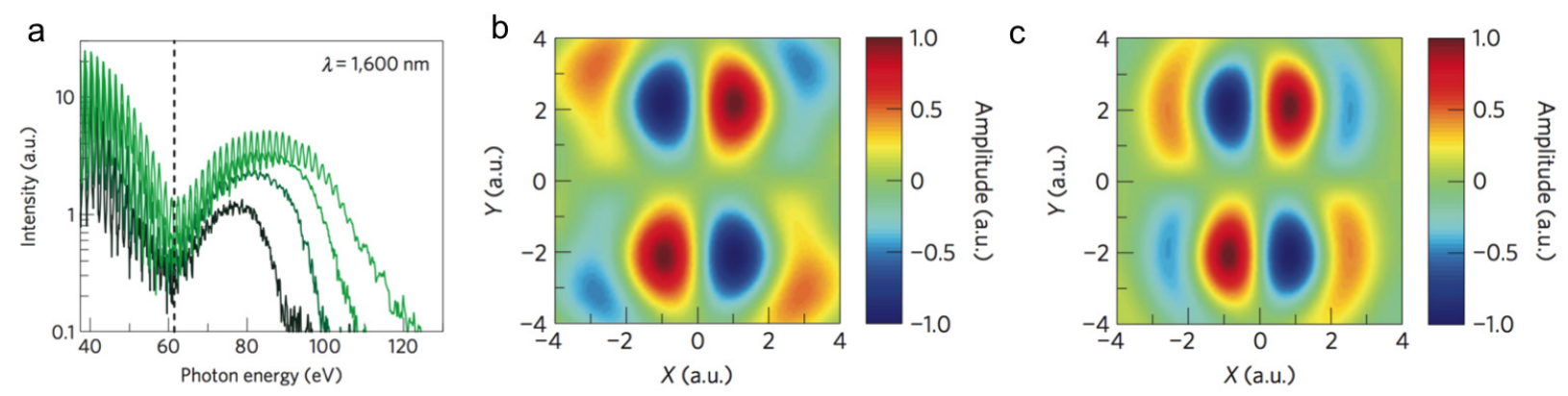

Figure 43: (a) Harmonic emission from $\mathrm{CO}_{2}$ molecules measured in correspondence of the maximum molecular alignment and for different laser intensities, in the range between 0.9 and $1.5 \times 10^{14} \mathrm{~W} / \mathrm{cm}^{2}$. (b) HOMO structure retrieved with the generalized tomographic procedure from the harmonic spectra acquired for different molecular orientations and (c) $2 \mathrm{D}$ projection of the HOMO calculated with a quantum chemistry program by taking into account the limited spatial frequencies sampled in the experiment. From ref. 455 with permission. Copyright 2011 Macmillan Publishers Limited.

of $\mathrm{CO}_{2}$, it was possible to estimate a distance between the two oxygen atoms of about 4.4 atomic units, which is in good agreement with the expected value. It is worth mentioning that the quality of the tomographic reconstruction depends on the extension of the generated harmonic spectra. Since the cutoff of the harmonic emission scales quadratically with the driving wavelength and linearly with the ionization potential of the molecule, all the studies conducted with Ti:Sapphire lasers have been limited to small molecules, which have relatively high $\mathrm{I}_{p}$. In the experiment by Vozzi et al., $\mathrm{HHG}$ in $\mathrm{CO}_{2}$ was driven by a tunable mid-IR laser source $(1.45-1.7 \mu \mathrm{m})$ and a noticeable extension of the harmonic emission was achieved, allowing the technique to be successfully extended to more complex molecules like $\mathrm{N}_{2} \mathrm{O}$ and acetylene. ${ }^{456}$

Shortly after the successful demonstration of HHG-based molecular tomography, it was experimentally ${ }^{457}$ and theoretically ${ }^{458}$ demonstrated that spectral features such as local minima can be also originated from the interference between different ionization channels during the first step of the HHG process (see also Section 6). The presence of such multi-electron effect was first evidenced by Wörner and co-authors from HHG measurements performed in $\mathrm{CO}_{2}$, where the position of the spectral minimum was found to depend on both the intensity and the wavelength of the driving pulse. ${ }^{459}$ However, the reported contributions from differ- 
ent harmonic generation pathways was not confirmed by measurements reported by Vozzi et $a l .{ }^{455}$ on the same system. This discrepancy was attributed to the shorter driving pulse duration and the lower peak intensity used in the latter experiment, for which a blurring of the dynamical interference occurred. The role of ionization from multiple orbitals and the interference between their contributions in $\mathrm{HHG}$ spectra was also investigated for $\mathrm{N}_{2}$ by performing experiments with a $1.2 \mu \mathrm{m}$ driving source and corroborated by a theory model

based on quantitative re-scattering theory (QRS). ${ }^{460}$ In this work, a strong dependence of the spectral minimum was observed as a function of the driving laser intensity. In particular, the disappearance of the minimum for high driving intensities was assigned by the QRS calculations to the contribution of the lower-lying orbital (HOMO-1) of the molecule.

\subsection{Laser-induced electron diffraction}

Recombination of the freed electronic wave packet with the parent molecular orbital (leading to HHG) is only one of the possible rescattering mechanisms arising from strong field ionization. Other physical processes can occur when the electron is driven back to the vicinity of the parent ion including (i) elastic scattering and (ii) electronic excitation of the parent ion (inelastic scattering). The elastically scattered electrons carry structural information on the target molecule: as the de Broglie wavelength of the returning wave packet becomes comparable to the molecular size, a diffraction pattern is produced in the electron momentum distribution (Fig. 44), from which the interatomic spacing can be determined. This imaging technique was dubbed laser-induced electron diffraction (LIED) and it was first proposed in 1996 by T. Zuo, A. D. Bandrauk and P. B. Corkum. ${ }^{184}$ As for HHG-based molecular tomography, this technique provides extreme temporal and spatial resolution. However, contrarily to conventional electron diffraction, molecular alignment relative to the laser polarization axis is required to extract useful information from the rescattering event. Moreover, since LIED is a strong-field process, the influence of the driving laser field has to be removed to allow one to extract the field-free information from the measured LIED momentum distribu- 


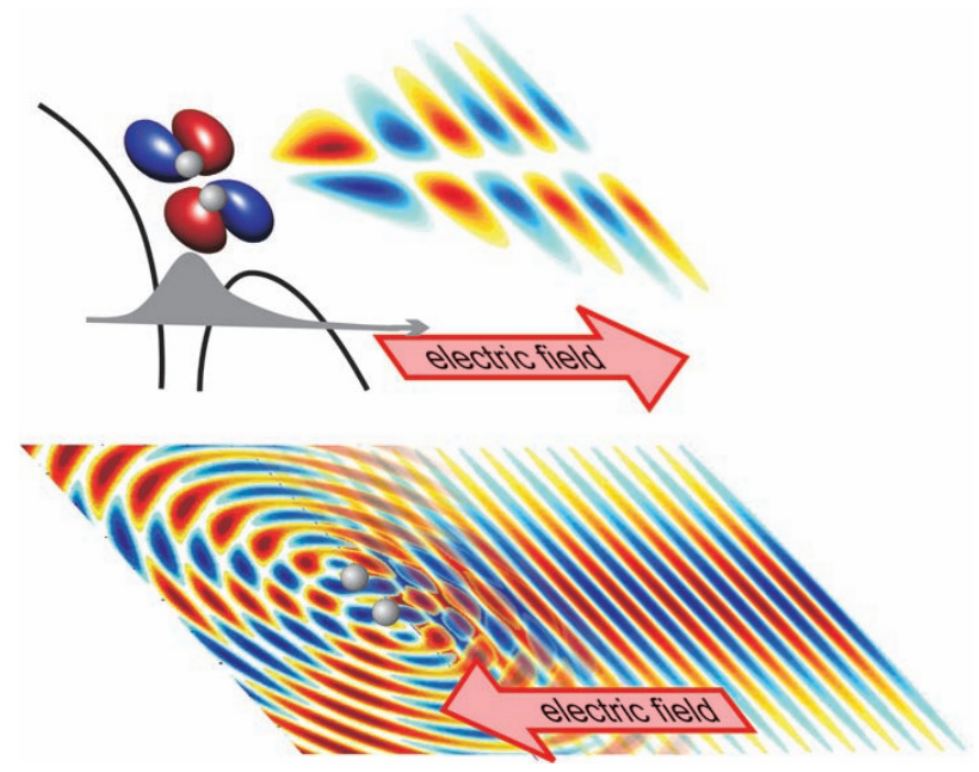

Figure 44: Schematic of LIED. Top panel: tunnel ionization - half of the wave packet goes directly to the detector. Bottom panel: recollision - the remaining part of the wave packet is driven back and it is diffracted from the molecule. From ref. 461 with permission. Copyright 2008 The American Association for the Advancement of Science (AAAS).

tion. To this end, the momentum distribution $\mathbf{p}$ is expressed as the sum of the momentum after recollision $\mathbf{p}_{r}$ and the vector potential at the instant of rescattering $\mathbf{A}_{r}$. Electrons detected along a circle of radius $\left|\mathbf{p}_{r}\right|$ provide a field-free signal comparable to the conventional electron diffraction.

The first successful experimental demonstration of LIED was published in 2008 by Meckel and co-workers. ${ }^{461}$ In this work, 800-nm pulses were used to ionize impulsively aligned $\mathrm{O}_{2}$ and $\mathrm{N}_{2}$ molecules. The electron momentum distributions for aligned and anti-aligned molecules were measured using the COLTRIMS technique. The measurements revealed not only the symmetry of the HOMO but also the interatomic dimensions of both $\mathrm{O}_{2}$ and $\mathrm{N}_{2}$ molecules. Further progress was subsequently achieved by Blaga et al by using a tunable mid-infrared source $(1.2-2.3 \mu \mathrm{m}) .{ }^{462}$ Increasing the driving wavelength with respect to that of traditional Ti-sapphire sources allows for a better spatial resolution, and a large momentum transfer is produced. With an improved spatial resolution, it has been possible to image the bond length adjustment of $\mathrm{O}_{2}$ following ionization: a contraction of the $\mathrm{O}-\mathrm{O}$ bond length of 


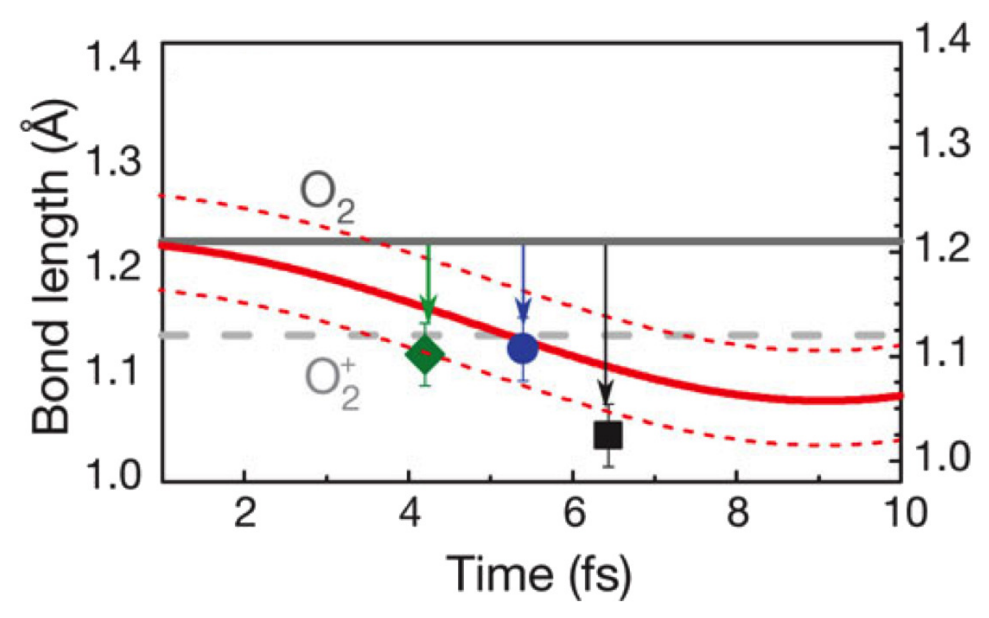

Figure 45: Bond lengths for $\mathrm{O}_{2}^{+}$extracted from the LIED measurements. Different wavelengths were used to scan different time delays between ionization and recollision. Square correspond to $2.3 \mu \mathrm{m}$, circle to $2 \mu \mathrm{m}$ and diamond to $1.7 \mu \mathrm{m}$. The measurement performed with the longer wavelength (longer time delay) indicate a clear contraction of the bond. The red curve shows the evolution of the nuclear wavepacket centre (solid) and its associated full-width at half-maximum (dotted), computed in the Frank-Condon approximation. The equilibrium bond lengths are indicated by the solid (neutral species) and dashed grey (ion) lines. The vertical arrows indicate that the measured bond lengths for all three wavelengths are consistently shorter $(\sim 0.1 \AA)$ than the equilibrium length for the neutral species and are statistically meaningful. From ref. 462 with permission. Copyright 2012 Macmillan Publishers Limited.

$0.1 \AA$ from the equilibrium distance of $1.21 \AA$ was extracted from the experimental data (Fig. 45). Very recently, Pullen et al exploited LIED to image a more complex molecule. In this case, a mid-infrared source $(3.1 \mu \mathrm{m})$ operating at high-repetition rate $(160 \mathrm{kHz})$ was used to perform LIED in aligned acetylene in combination with coincidence detection (COLTRIMS). ${ }^{463,464}$ This sophisticated experimental setup has allowed the $\mathrm{C}-\mathrm{C}$ and $\mathrm{C}-$ $\mathrm{H}$ bond lengths to be simultaneously retrieved. The experiment also confirmed that no significant structural rearrangements occur after acetylene is ionized, and the bond lengths for both $\mathrm{C}-\mathrm{C}$ and $\mathrm{C}-\mathrm{H}$ were found to be constant in the time interval of recollision. By using a similar technique, Wolter at $\mathrm{al}^{465}$ have recently obtained snapshots of the bond breaking in the di-ionized acetylene and, by introducing an additional laser field, have been able to control this ultrafast dissociation process. 


\subsection{Time-Resolved Holography with Photoelectrons}

Besides HHG and LIED, laser-driven electron recollision can lead to another intriguing process: photoelectron holography. The key aspect of holography is the observation of an interference pattern originated from a reference wave and a signal wave. ${ }^{466}$ Both waves are emitted by the same coherent source and the signal wave is scattered off a target. Due to the path-length differences, a phase difference $\Delta \phi=\left(k-k_{z}\right) z_{0}$ is accumulated between the two waves (where $k$ is the total momentum, $k_{z}$ is the momentum in the $z$ direction, and $z_{0}$ is the distance to the scattering target), thus producing interference on the detector. This interference pattern carries information on the target structure. This condition can be accomplished with tunnel ionization from an atomic or molecular target: the produced electronic wave function is the sum of a reference wave packet and a signal wave packet which oscillates in the laser field and scatters off the parent ion. In the strong field approximation the phase difference between the two electronic waves can be written as

$$
\Delta \phi \approx p_{r}^{2}\left(t_{C}-t_{0}^{r e f}\right) / 2
$$

where $p_{r}$ is the momentum perpendicular to the laser polarization axis, $t_{C}$ is the time when the signal wave packet scatters off the ion, and $t_{r e f}$ is the time of birth of the reference wave packet. The hologram encodes changes in the scattering potential in the time interval between $t_{r e f}$ and $t_{C}$, as well as the relative change in ionization rates. Thus, photoelectron holography can be seen as a pump-probe technique providing sub-femtosecond time resolution.

The first experimental demonstration of time-resolved holography was performed in 2011

by Huismans et al. ${ }^{467}$ In this experiment, a mid-IR (carrier wavelength $7 \mu \mathrm{m}$ ) FEL was used to tunnel ionize metastable (6s) Xe atoms. Angle resolved photoelectron spectra were recorded with a VMI integrated into the FEL laser cavity. The peak intensity of the laser was varied by changing the position of the apparatus along the laser propagation axis. 
The acquired images exhibit the typical patterns related to holographic interference (Fig. 46(a)). The experimental observation of holographic interferences was further corroborated by calculations based on the time dependent Schrödinger equation (TDSE), which showed similar fringe patterns (Fig. 46(b)). The main features of the hologram were reproduced also by semiclassical calculations with the Coulomb-corrected strong-field approximation (CCSFA) 468 (Fig. 46(b)). These calculations allowed to trace quantum trajectories taking into account the Coulomb interaction of the electron. The combination of the experimental and theoretical results has allowed demonstrating that the hologram stores spatial and temporal information about the core- and electron dynamics.

Following this encouraging result, photoelectron holography was subsequently extended to molecules by Meckel and co-workers. ${ }^{469}$ In this experiment, an aligned ensemble of $\mathrm{N}_{2}$ molecules was tunnel ionized by using a Ti:Sapphire laser producing 800-nm, 40-fs, $5-\mu \mathrm{J}$ pulses with a repetition rate of $30 \mathrm{kHz}$. Due to the relatively short laser wavelength, holography conditions were met by increasing the peak intensity of the driving pulses to $1.3 \times$ $10^{14} \mathrm{~W} / \mathrm{cm}^{2}$. Photoelectrons were detected in coincidence with $\mathrm{N}_{2}^{+}$ions exploiting a reaction microscope. Both the experimental results and a sophisticated theoretical analysis indicated that the holographic interference depends on the molecular orientation. In particular, a shift of the holographic fringes is caused by an effective offset of the centre of phase curvature of the incident wavepacket. These important findings evidenced that strong-field recollision in molecules cannot be simply described as a molecule-centered partial-wave diffraction experiment.

\subsection{Multichannel photoemission in strong field ionization}

All techniques described in this section are based on the primary ionization event induced by the field. It was long assumed, based on the inverse exponential dependence of tunneling amplitude on the ionization potential predicted by tunneling models, that only the HOMO ionization played a significant role. It is clearly the case for the tomographic reconstruction 

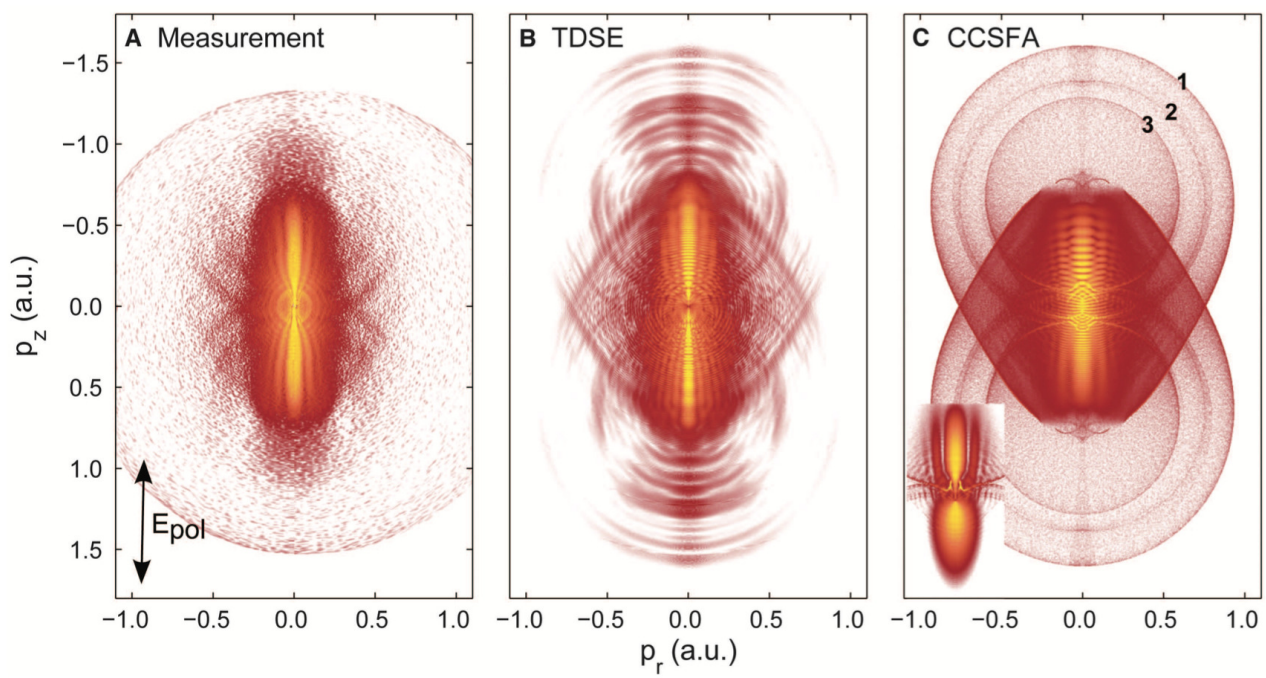

Figure 46: Experimental (a) and calculated (b-c) VMI photoelectron images. (a) Holographic interference measured from the ionization of metastable Xe atoms. (b) TDSE calculation for ionization of argon $(5 \mathrm{~s})$ and (c) CCSFA calculation for ionization of a model hydrogenic atom. From Ref. 467 with permission. Copyright 2011 The American Association for the Advancement of Science (AAAS).

of the HOMO (Dyson) orbital discussed in section 7.1. In general, this is a reasonable assumption for small molecules, where the energy gap between HOMO and HOMO-1 is relatively large, although it may be strongly dependent on the orientation of the electric vector, and the shape of the orbitals. ${ }^{470}$ However, as mentioned above, contributions to ionization from deeper orbitals has been repeatedly put in evidence. It is obvious that as the molecular size and the density of valence states increase, contributions from a manifold of outer ionic states becomes important, and will complicate analysis of the experiments.

Disentangling contributions of electrons emitted from different orbitals is not easy, as each ionization produces an ATI comb, which adds up in the total spectrum, and requires some type of coincidence detection to be separated in the total spectrum. A study in this direction was performed by Boguslavskiy et al. ${ }^{471}$ on strong field ionization of the two hydrocarbons n-butane and 1,3-butadiene. Exploiting the known fragmentation pattern of the various ionic states, notably the fact that no fragmentation is produced by HOMO ionization, correlation between photoelectron and mass selected ions was measured. From these data they were able to separate the various ATI combs correlating with each fragmentation pattern, and 
confirmed their origin by the energy shifts between the maxima, which match, modulo the photon energy, the energy differences between the ionization potentials of the different ionic states, corrected for the field effects (Stark shifts and ponderomotive energy). The results showed conclusively that strong field ionization takes place from several initial orbitals. In 1,3-butadiene ionization out of deeper orbitals is minor, as is expected from the large energy gap, over $2 \mathrm{eV}$, between HOMO and HOMO-1 ionizations. In contrast, in n-butane ionization from inner orbitals is predominant, as energy separation is much smaller, and other factors, like orbital shape and nodal structure, become important. The match of energy shift between different photoelectron combs with the IP difference could also rule out production of deeper molecular ion states by excitation of the ground state ion by the field, subsequent to the emission. This effect, dubbed nonadiabatic multielectron (NME) ionization, was also studied by simulations that included or suppressed this effect. While at low intensity $\left(1.5 \times 10^{13} \mathrm{~W} \mathrm{~cm}^{-2}\right)$ the results were in good agreement with experiment, at higher intensity $\left(1.5 \times 10^{14} \mathrm{~W} \mathrm{~cm}^{-2}\right) \mathrm{NME}$ effects turned out very relevant, and orientation dependent. Although coincidence experiments become much more complex, the possibility of disentangling electron ionization from different initial orbitals is extremely important for the correct analysis of the imaging techniques considered, and opens the door to access similar information for deeper ionic states.

A further study extending the measurement to angle dependent ionization relative to the different channels has been presented in, ${ }^{472}$ and to full 3D photoelectron momentum detection, employing a reaction microscope, in. ${ }^{473}$ An alternative technique to select ionization out of a deeper orbital has been implemented by Yao at al ${ }^{474}$ monitoring fluorescence due to radiative decay of excited ionics states. They studied fluorescence relative to the $\mathrm{A} \rightarrow \mathrm{X}$ and $\mathrm{B} \rightarrow \mathrm{X}$ transitions in $\mathrm{CO}_{2}^{+}$as a function of the angle of the molecular axis and the ionizing field polarization. By this they could retrieve angle dependent ionization yield relative to HOMO$1\left(1 \pi_{u}\right)$ and HOMO-2 $\left(3 \sigma_{u}\right)$ ionizations. The laser intensity of $\sim 4 \times 10^{14} \mathrm{~W} \mathrm{~cm}^{-2}$ ensures the tunneling ionization regime also for the inner ionizations (Keldysh $\gamma \sim 0.6$ ). Similar to the 
older total ionization yield study of Pavičić et al. ${ }^{475}$ they observed a significant disagreement with the MO-ADK prediction, while agreement was excellent with SFA (length). For HOMO-2 ionization, both MO-ADK end SFA gave very similar results, although sharper than the experiment. Caution should be exercised, however, with MO-ADK results from analysis of MOs obtained by Quantum Chemistry GTO calculations, as the rapid decrease of gaussian functions may give significant errors in the tails of the weve functions, as noticed just in $\mathrm{CO}_{2} \mathrm{HOMO}$ ionization. ${ }^{476,477}$ The technique is remarkable for its effectiveness and simplicity, although it is of course blind to HOMO ionization.

\section{Conclusion and outlook}

Since its birth at the beginning of the twenty first century, attosecond technology has provided a number of tools that allows one to observe the ultrafast motion of electrons in a variety of quantum systems, such as atoms, molecules, nanostructures and solids. In particular, the possibility to precisely control electron dynamics in complex molecules by using these techniques has attracted the attention of many scientists, since this has opened the way to new forms of doing chemistry, usually gathered under the heading "attochemistry". In this review, we have discussed the application of attosecond pulses to the investigation of ultrafast processes in molecular systems, with particular emphasis on polyatomic molecules. The ability to initiate and measure electron dynamics in complex molecular structures represents a crucial step forward in pursuing the goal of manipulating chemical reactions by exclusively acting on the particles that are ultimately responsible for chemical bonding: the electrons. For example, the experimental observation of ultrafast processes on the attosecond time scale can open new perspectives in the understanding of the physics governing electron transport of biological signals. Also, the ability to visualize charge dynamics occurring in molecular subunits can be of primary importance to unravel the role of the electrons in the subsequent structural changes that a biomolecule undergoes. All this might open new 
perspectives for the development of new technologies, for example in molecular electronics, where electron processes on an ultrafast temporal scale are essential to trigger and control the electron current on the scale of the molecule.

This rapid development of attosecond science would not have been possible without the help of theory. Theoretical modeling and numerical simulations have been crucial for the design of new experimental schemes and for the interpretation of the complex outcomes of the measurements. However, performing fully ab initio theoretical calculations to describe the correlated motion of electrons and nuclei that follows ionization of a molecule by attosecond pulses is still very challenging. Indeed, besides the intrinsic limitations associated with the large number of electronic and nuclear degrees of freedom, there is the additional complication of describing the electronic continuum of the molecule, which cannot be done by using standard quantum chemistry methods. Broadband attosecond pulses create molecular ions in several (usually many) excited states, in which nuclear wave packets are launched simultaneously and coherently; after a few femtoseconds, the overall molecular dynamics is strongly affected by the coupling between electronic and nuclear degrees of freedom, causing the breakdown of the Born-Oppenheimer approximation and hence an increase in the number of channels participating in the dynamics. So far, an accurate full quantum mechanical description of all these effects, from the ionization step to the subsequent evolution of the system, has only been achieved for the simplest molecules $\left(\mathrm{H}_{2}^{+}, \mathrm{H}_{2}\right.$ and $\left.\mathrm{D}_{2}\right)$. In comparison with this, theoretical investigations in large molecules can be considered in their infancy. Accurate methods already exist to describe ionization of diatomic and small polyatomic molecules, and more approximate ones work reasonably well for larger molecules. However, the description of the dynamics that comes after ionization is still far from being accomplished. This review describes the theoretical approaches most commonly used for polyatomic molecules and how, even though their current limitations, they have been able to guide experimental efforts on these systems.

Despite the impressive progress in attosecond science, formidable challenges must still be 
faced by the attosecond community in the near future. For example, in most pump-probe schemes applied to investigations of ultrafast dynamical processes in molecules, in particular large molecules, IR probe pulses have been used. However, as we have seen, the dynamics launched by the attosecond pump pulse may be partly hidden, if not totally obscured, by that induced by the IR probe field. This effect is particularly important in current pump-probe experiments since the intensities of the IR probe pulses are strong enough to significantly distort the molecular potential. A possible way out to explore field-free molecular dynamics is the use of "weak" EUV pulses for both the pump and the probe, ${ }^{402,404}$ because their short wavelength ensures that the ponderomotive energy, i.e., the energy acquired by an electron due to its interaction with the external field, is negligible in comparison with that associated with the electron-electron and electron-nucleus interactions. So far, EUV-only pump-probe spectroscopy of electron dynamics has only been demonstrated in a few relevant cases, namely in $\mathrm{Xe}^{207}$ and $\mathrm{H}_{2},{ }^{210}$ where electron dynamics was recorded with a time resolution of the order of $1 \mathrm{fs}$. The key point in these experiments is the use of EUV pulses produced by HHG, short enough $(\sim 1 \mathrm{fs})$ to ensure high temporal resolution. Still, EUV pulses generated in this way are so weak that measurements are generally affected by a rather low statistics. Progress towards significantly increasing the intensity of these EUV pulses has been recently reported in the literature. ${ }^{206,400}$

An alternative to pulses generated from high-harmonic emission is the use of EUV pulses produced by free electron lasers (FEL). For these pulses, intensities are substantially higher, though still low enough to avoid distortions of the molecular potential. The main problem here is to achieve the necessary sub-fs duration of the pulses, as well as to get rid of their stochastic character to ensure the reproducibility of the generated pulses. First attempts towards EUV-only attosecond pump-probe experiments are currently under way.

Another point that will receive a considerable attention in the near future is to investigate if available attosecond techniques, successfully used to investigate electron dynamics in isolated atomic systems, can provide similarly valuable information on the dynamics of 
large molecules. As explained in this review, the most widely used techniques in atoms and small molecules, apart from attosecond pump-probe spectroscopy, are attosecond transient absorption spectroscopy (ATAS), reconstruction of attosecond beating by interference of two-photon transitions (RABBITT), attosecond streak camera, high-harmonic spectroscopy, core-hole Auger spectroscopy, and the attoclock. All of them have provided deep insights on the ultrafast electron dynamics that follows ionization of atoms by an attosecond pulse, from the measurement of photoelectron emission time delays to the full reconstruction of the electronic wave packet generated by the pulse. The latter is the ultimate goal of any attosecond technique, since it opens the way to fully control electron dynamics. However, their performance to investigate electron dynamics in large molecules remains to be seen due to the many additional degrees of freedom that operate in this case: nuclear motion, intermolecular interactions, solvent effects and environment. Studying the feasibility of such techniques in the context of large molecules should be the object of experimental research in the forthcoming years.

The complete analysis of the ultrafast dynamics in many-electron molecular systems will also require the development of highly sophisticated detection techniques able to provide a more complete characterization of the observed dynamics. These will include multicoincidence measurements, ideally those able to provide a complete kinematic description of the observed process (e.g., COLTRIMS, VMI, etc), multidimensional X-ray spectroscopies, and attosecond imaging techniques such as time-resolved molecular tomography and/or timeresolved holography. Also the extension of attosecond pulse generation to the multi-keV photon energy region would open the way to attosecond x-ray diffraction experiments in complex systems, thus giving the possibility to combine the extreme temporal resolution offered provided by attosecond pulses with picometer spatial resolution.

From the theory side, the road towards complex molecular structures requires an accurate description of the interaction of ultrashort pulses with molecular systems composed by tens of atoms and the calculation of the subsequent dynamics. The first steps towards achieving 
the first part have been amply described in this review. However, theory is still far from being able to describe how the electron dynamics that follows the interaction with an attosecond pulse is affected by the coupling with the nuclear degrees of freedom, and how this coupling determines the fate of the system in the form of charge localization and the production of specific molecular fragments. Also, production of secondary electrons resulting from Auger decay, which has been recognized as a very important source of cellular damage, has been ignored in most theoretical studies involving large molecules due to the difficulty (i) to describe the ionization continuum, this time containing multiple electrons, (ii) to account for correlation effects in that continuum, and (iii) to describe the coupling with the nuclear motion, which occurs in a similar time scale as Auger emission. Efforts devoted to fill this void in large molecules should keep theorists busy in the next few years. Significant progress in this direction is really necessary to get a complete understanding of how electron dynamics dictates the chemical behavior of large molecules at longer time scales where chemistry happens and how it can be used to alter this chemistry.

Particularly challenging for theory is also the description of real pump-probe experiments, in which the probe pulse can actively modify the electron dynamics initiated by the pump pulse, for example by generating a coupling between different states with an energy difference that is favorable for one or two photon transitions, thus inducing population transfers before molecular fragmentation. Although this has been achieved for the $\mathrm{H}_{2}$ molecule and other diatomics, theory is still far away from providing a similar level of description for large molecules, since the use of a probe pulse, even an IR one, inevitably leads to additional ionization of the system and, very likely, to Auger decay.

In conclusion, in this review we have tried to convey to the reader the message that attosecond science is rapidly progressing towards the investigation and understanding of complex ultrafast dynamics in large molecular systems, with important implications in a variety of research fields, going from physics, passing through chemistry, and arriving recently to biology. However, there is still a long way to fully exploit the capabilities that attosecond 
techniques can offer in order to warrant their solid establishment in chemistry and biology, but the prospects for the years to come are excellent.

\section{Biographies}

Mauro Nisoli was born in Bergamo, Italy, in 1965. From 1990 to 2000, he was a Researcher of the National Research Council (CNR) with the Center of Quantum Electronic and Electronic Instrumentation. From 2001 to 2010 he was an Associate Professor with the Department of Physics of Politecnico di Milano, where he is now Full Professor. He is author of about 180 research papers in international journals, 1 patent, several invited and tutorial communications at international meetings and schools. His research focus are the following: attosecond science, ultrashort-pulse laser technology, control and real-time observation of electronic motion in atoms and molecules. Prof. Nisoli was awarded an European Research Council (ERC) Advanced Grant in 2009.

Piero Decleva was at the Department of Chemistry, University of Trieste, where he became a Lecturer in theoretical chemistry in 1976, an Associate Professor of quantum chemistry from 1982 to 1994, and then a Full Professor of chemical physics till present. He is author of 240 research papers in international journals. His research covered theoretical description of many-body effects and electron cross sections in molecular photoionization, with many collaborations with leading experimental groups. A large molecular code for the calculation of dynamical photoionization observables has been developed in Trieste, with recent emphasis on ultrashort time dependent phenomena and strong field ionization.

Francesca Calegari received the Ph.D. from Politecnico di Milano in 2009. She was Post-

doctoral Researcher with CNR-INFM until 2010 and with Politecnico di Milano until 2011. From 2011 till 2016 she was Staff Researcher at CNR-IFN and Adjunct Professor of Physics at Politecnico di Milano. In 2014 she was Visiting Scientist at MPSD, Hamburg, Germany 
and the same year she was awarded an European Research Council (ERC) Starting Grant to investigate the role of the electron dynamics in the photochemistry of biomolecules. In 2015 she was awarded a "special recognition to young women in Photonics" by the European Optical Society (EOS). Since 2016 she has been appointed Full Professor of Physics at the University of Hamburg and Leading Scientist at the Deutsches Elektronen-Synchrotron where she leads the Attosecond Science Division. The main focus of her research is to track and ideally control in real time the electron dynamics occurring in systems with increasing complexity from simple molecules to molecules of biological interest and nanostructured materials.

Alicia Palacios was born in Tomelloso, Spain (1979). She received the Graduate degree in chemistry in 2001 and the Ph.D. degree in 2006, both from the Universidad Aut Assnoma de Madrid, Spain. She completed her education with several stays in the Universit $\tilde{A} l$ Bordeaux I, and a long-term Postdoctoral fellowship (2006âĂŞ2009) in Lawrence Berkeley National Lab, Berkeley, CA, USA. She has now a Tenure Track position (RamÂşn y Cajal) in Madrid. She received the Marie Curie Integration Grant (ATTOTREND) as a Fellow Researcher. She has published more than 45 peer reviewed articles in international journals working on atomic and molecular physics, in particular, developing new ab initio time-dependent treatments for the description of atoms and molecules subject to ultrashort intense laser pulses.

Fernando Martín was born in Madrid, Spain, in 1961. He graduated in Chemistry in 1984 and Physics in 1986 at the Universidad Autónoma de Madrid. He received his PhD Degree at the same university in 1986. He completed his postdoctoral studies at the University of Bordeaux (1988), the Université de Paris VI (1989-1990) and the Univesity of Chicago (19951996). He is Full Profesor at the Universidad Autónoma de Madrid since 2005. His research focuses on the theoretical modeling of photoexcitation and photoionization of atomic and molecular systems induced by synchrotron radiation and ultrashort laser pulses, as well as 
of complex molecular systems, isolated or deposited on surfaces. He has published over 350 articles and, since 2011, he is recipient of an Advanced Grant from the European Research Council.

\section{Acknowledgement}

We acknowledge the support from the European Research Council under the ERC grants 227355 ELYCHE, 290853 XCHEM, and 637756 STARLIGHT, the European COST Action CM1204 XLIC, the MINECO Project No. FIS2013-42002-R, and the CAM project NANOFRONTMAG. Theoretical results shown in Figs. 25, 26, 27, 28, 40, and 41 were obtained at the Mare Nostrum BSC and CCC-UAM computer centers. 


\section{Abbreviations}

\begin{tabular}{|c|c|}
\hline $\mathrm{ADC}$ & Algebraic Diagrammatic Construction \\
\hline $\mathrm{APT}$ & Attosecond Pulse Train \\
\hline ARPES & Angle Resolved Photoemission Spectroscopy \\
\hline CASSCF & Complete Active Space Self-Consistent Field \\
\hline $\mathrm{CC}$ & Close-Coupling \\
\hline CC-LR & Coupled Cluster-Linear Response \\
\hline CEP & Carrier-Envelope Phase \\
\hline $\mathrm{CI}$ & Configuration Interaction \\
\hline CIS & Configuration Interaction Singles \\
\hline CMS & Continuum Multiple-Scattering \\
\hline COLTRIMS & COLd-Target Recoil Ion Momentum Spectroscopy \\
\hline $\mathrm{CPA}$ & Chirped-Pulse Amplification \\
\hline $\mathrm{CW}$ & Coulomb Wave \\
\hline DFG & Difference Frequency Generation \\
\hline DFT & Density Functional Theory \\
\hline DOG & Double Optical Gating \\
\hline $\mathrm{EOM}$ & Equation Of Motion \\
\hline EUV & Extreme UltraViolet \\
\hline $\mathrm{EOM}-\mathrm{CC}$ & Equation-Of-Motion Coupled Cluster \\
\hline FEL & Free Electron Laser \\
\hline FFT & Fast Fourier Transform \\
\hline FROG & Frequency Resolved Optical Gating \\
\hline FROG CRAB & FROG for Complete Reconstruction of Attosecond Bursts \\
\hline GDOG & Generalized DOG \\
\hline GTO & Gaussian-Type Orbital \\
\hline
\end{tabular}




$\begin{array}{ll}\text { HCF } & \text { Hollow-Core Fiber } \\ \text { HF } & \text { Hartree Fock } \\ \text { HHG } & \text { High-order Harmonic Generation } \\ \text { IG } & \text { Ionization Gating } \\ \text { IP } & \text { Ionization Potential } \\ \text { IPG } & \text { Interferometric Polarization Gating } \\ \text { IR } & \text { InfraRed } \\ \text { KS } & \text { Kohn-Sham } \\ \text { KT } & \text { Koopmans Theorem } \\ \text { LCAO } & \text { Linear Combination of Atomic Orbitals } \\ \text { LIED } & \text { Laser Induced Electron Diffraction } \\ \text { LR } & \text { Linear Response } \\ \text { MCP } & \text { Micro-Channel Plate } \\ \text { MCTDH } & \text { MultiConfiguration Time-Dependent Hartree } \\ \text { MFPAD } & \text { Molecular Frame Photoelectron Angular Distribution } \\ \text { MO } & \text { Molecular Orbital } \\ \text { MRCI } & \text { Multireference Configuration Interaction } \\ \text { MT } & \text { Muffin Tin } \\ \text { NIR } & \text { Near InfraRed } \\ \text { NOG } & \text { Noncollinear Optical Gating } \\ \text { NWP } & \text { Nuclear Wave Packet } \\ \text { OCE } & \text { One-Center Expansion } \\ \text { OCW } & \text { Orthogonalized Coulomb Wave } \\ \text { OPA } & \text { Optical Parametric Amplifier } \\ \text { OPCPA } & \text { Optical Parametric Chirped-Pulse Amplification } \\ & \end{array}$


OPW Orthogonalized Plane Wave

PACER Probing Attosecond dynamics by Chirp-Encoded Recollision

PEC Potential Energy Curve

PCGPA Principal Component Generalized Projection Algorithm

PROOF Phase Retrieval by Omega Oscillation Filtering

PW Plane Wave

RASSCF Restricted Active Space Self Consistent Field

RAS Resonant Auger Spectroscopy

RPA Random Phase Approximation

RABBITT Reconstruction of Attosecond Beating By Interference of Two-photon Transitions

RASSCF Restricted Active Space Self Consistent Field

REMI REaction MIcroscope

SA Sudden Approximation

SAC-CI Symmetry Adapted Cluster-Configuration Interaction

SAE Single Active Electron

SCF Self-Consistent Field

$\triangle \mathrm{SCF} \quad$ Delta Self-Consistent Field

SFA Strong Field Approximation

STO Slater-Type Orbital

TDA Tamm-Dancoff Approximation

TDDFT Time-Dependent Density Functional Theory

TDSE Time-Dependent Schrödinger Equation

TOF Time-Of-Flight

TRPES Time-Resolved PhotoEmission Spectroscopy

VMI Velocity Map Imaging

XAS X-ray Absorption Spectroscopy

WP Wave Packet 


\section{References}

(1) Donoso, A.; Martens, C. C. Simulation of Coherent Nonadiabatic Dynamics Using Classical Trajectories. J. Phys. Chem. A 1998, 102, 4291-4300.

(2) Zewail, A. H. Femtochemistry: Atomic-Scale Dynamics of the Chemical Bond. J. Phys. Chem. A 2000, 104, 5660-5694.

(3) Krausz, F.; Ivanov, M. Attosecond Physics. Rev. Mod. Phys. 2009, 81, 163-234.

(4) Lépine, F.; Ivanov, M. Y.; Vrakking, M. J. J. Attosecond Molecular Dynamics: Fact or Fiction? Nat. Photonics 2014, 8, 195-204.

(5) Golubev, N. V.; Kuleff, A. I. Control of Charge Migration in Molecules by Ultrashort Laser Pulses. Phys. Rev. A 2015, 91, 051401.

(6) Paul, P. M.; Toma, E. S.; Breger, P.; Mullot, G.; Augé, F.; Balcou, P.; Muller, H. G.; Agostini, P. Observation of a Train of Attosecond Pulses from High Harmonic Generation. Science 2001, 292, 1689-1692.

(7) Hentschel, M.; Kienberger, R.; Spielmann, C.; Reider, G. A.; Milosevic, N.; Brabec, T.; Corkum, P.; Heinzmann, U.; Drescher, M.; Krausz, F. Attosecond Metrology. Nature 2001, 414, 509-513.

(8) Drescher, M.; Hentschel, M.; Kienberger, R.; Uiberacker, M.; Yakovlev, V.; Scrinzi, A.; Westerwalbesloh, T. T.; Kleineberg, U.; Heinzmann, U.; Krausz, F. Time-Resolved Atomic Inner-Shell Spectroscopy. Nature 2002, 419, 803-807.

(9) Goulielmakis, E.; Loh, Z.-H.; Wirth, A.; Santra, R.; Rohringer, N.; Yakovlev, V. S.; Zherebtsov, S.; Pfeifer, T.; Azzeer, A. M.; Kling, M.; et al., Real-Time Observation of Valence Electron Motion. Nature 2010, 466, 739-743. 
(10) Mauritsson, J.; Remetter, T.; Swoboda, M.; Klünder, K.; L'Huillier, A.; Schafer, K. J.; Ghafur, O.; Kelkensberg, F.; Siu, W.; Johnsson, P.; et al., Attosecond Electron Spectroscopy Using a Novel Interferometric Pump-Probe Technique. Phys. Rev. Lett. 2010, 105,053001 .

(11) Schultze, M.; Fieß, M.; Karpowicz, N.; Gagnon, J.; Korbman, M.; Hofstetter, M.; Neppl, S.; Cavalieri, A. L.; Komninos, Y.; Mercouris, T.; et al., Delay in Photoemission. Science 2010, 328, 1658-1662.

(12) Klünder, K.; Dahlström, J. M.; Gisselbrecht, M.; Fordell, T.; Swoboda, M.; Guénot, D.; Johnsson, P.; Caillat, J.; Mauritsson, J.; Maquet, A.; et al., Probing Single-Photon Ionization on the Attosecond Time Scale. Phys. Rev. Lett. 2011, 106, 143002.

(13) Ott, C.; Kaldun, A.; Argenti, L.; Raith, P.; Meyer, K.; Laux, M.; Zhang, Y.; Blättermann, A.; Hagstotz, S.; Ding, T.; et al., Reconstruction and Control of a TimeDependent Two-Electron Wave Packet. Nature 2014, 516, 374-378.

(14) Gruson, V.; Barreau, L.; Jiménez-Galan, Á.; Risoud, F.; Caillat, J.; Maquet, A.; Carré, B.; Lepetit, F.; Hergott, J.-F.; Ruchon, T.; et al., Attosecond Dynamics through a Fano Resonance: Monitoring the Birth of a Photoelectron. Science 2016, 354, 734738.

(15) Kaldun, A.; Blättermann, A.; Stooß, V.; Donsa, S.; Wei, H.; Pazourek, R.; Nagele, S.; Ott, C.; Lin, C. D.; Burgdörfer, J.; et al., Observing the Ultrafast Buildup of a Fano Resonance in the Time Domain. Science 2016, 354, 738-741.

(16) Dudovich, N.; Smirnova, O.; Levesque, J.; Mairesse, Y.; Ivanov, M. Y.; Villeneuve, D. M.; Corkum, P. B. Measuring and Controlling the Birth of Attosecond XUV Pulses. Nat. Phys. 2006, 2, 781-786. 
(17) Uiberacker, M.; Uphues, T.; Schultze, M.; Verhoef, a. J.; Yakovlev, V.; Kling, M. F.; Rauschenberger, J.; Kabachnik, N. M.; Schröder, H.; Lezius, M.; et al., Attosecond Real-Time Observation of Electron Tunnelling in Atoms. Nature 2007, 446, 627-32.

(18) Pfeiffer, A. N.; Cirelli, C.; Smolarski, M.; Dimitrovski, D.; Abu-samha, M.; Madsen, L. B.; Keller, U. Attoclock Reveals Natural Coordinates of the Laser-Induced Tunnelling Current Flow in Atoms. Nat. Phys. 2012, 8, 76-80.

(19) Cavalieri, a. L.; Müller, N.; Uphues, T.; Yakovlev, V. S.; Baltuska, a.; Horvath, B.; Schmidt, B.; Blümel, L.; Holzwarth, R.; Hendel, S.; et al., Attosecond Spectroscopy in Condensed Matter. Nature 2007, 449, 1029-1032.

(20) Schultze, M.; Bothschafter, E. M.; Sommer, A.; Holzner, S.; Schweinberger, W.; Fiess, M.; Hofstetter, M.; Kienberger, R.; Apalkov, V.; Yakovlev, V. S.; et al., Controlling Dielectrics with the Electric Field of Light. Nature 2013, 493, 75-78.

(21) Schultze, M.; Ramasesha, K.; Pemmaraju, C. D.; Sato, S. A.; Whitmore, D.; Gandman, A.; Prell, J. S.; Borja, L. J.; Prendergast, D.; Yabana, K.; et al., Attosecond Band-Gap Dynamics in Silicon. Science 2014, 346, 1348-1351.

(22) Locher, R.; Castiglioni, L.; Lucchini, M.; Greif, M.; Gallmann, L.; Osterwalder, J.; Hengsberger, M.; Keller, U. Energy-Dependent Photoemission Delays from Noble Metal Surfaces by Attosecond Interferometry. Optica 2015, 2, 405-410.

(23) Sommer, A.; Bothschafter, E. M.; Sato, S. A.; Jakubeit, C.; Latka, T.; Razskazovskaya, O.; Fattahi, H.; Jobst, M.; Schweinberger, W.; Shirvanyan, V.; et al., Attosecond Nonlinear Polarization and Light-Matter Energy Transfer in Solids. Nature 2016, 534, 86-90.

(24) Neppl, S.; Ernstorfer, R.; Cavalieri, A. L.; Lemell, C.; Wachter, G.; Magerl, E.; Bothschafter, E. M.; Jobst, M.; Hofstetter, M.; Kleineberg, U.; et al., Direct Observation 
of Electron Propagation and Dielectric Screening on the Atomic Length Scale. Nature 2015, 517, 342-346.

(25) Calegari, F.; Ayuso, D.; Trabattoni, A.; Belshaw, L.; De Camillis, S.; Anumula, S.; Frassetto, F.; Poletto, L.; Palacios, A.; Decleva, P.; Greenwood, J. B.; et al., Ultrafast Electron Dynamics in Phenylalanine Initiated by Attosecond Pulses. Science 2014, $346,336-339$.

(26) Sansone, G.; Calegari, F.; Nisoli, M. Attosecond Technology and Science. J. Sel. Top. Quantum Electron 2012, 18, 507-519.

(27) Sánchez, I.; Martín, F. Origin of Unidentified Structures in Resonant Dissociative Photoionization of $H_{2}$. Phys. Rev. Lett. 1997, 79, 1654-1657.

(28) Sánchez, I.; Martín, F. Resonant Dissociative Photoionization of $\mathrm{H}_{2}$ and $\mathrm{D}_{2}$. Phys. Rev. A 1998, 57, 1006-1017.

(29) Palacios, A.; Bachau, H.; Martín, F. Step-Ladder Rabi Oscillations in Molecules Exposed to Intense Ultrashort VUV Pulses. Phys. Rev. A 2006, 74, 031402(R).

(30) Sanz-Vicario, J.; Bachau, H.; Martín, F. Time-Dependent Theoretical Description of Molecular Autoionization Produced by Femtosecond XUV Laser Pulses. Phys. Rev. A 2006, 73, 033410.

(31) Sanz-Vicario, J.; Palacios, A.; Cardona, J.; Bachau, H.; Martin, F. Ab initio TimeDependent Method to Study the Hydrogen Molecule Exposed to Intense Ultrashort Laser Pulses. J. Electron. Spectrosc. Relat. Phenom. 2007, 161, 182-187.

(32) Palacios, A.; Sanz-Vicario, J. L.; Martín, F. Theoretical Methods for Attosecond Electron and Nuclear Dynamics: Applications to the $\mathrm{H}_{2}$ Molecule. J. Phys. B: At., Mol. Opt. Phys.: At., Mol. Opt. Phys. 2015, 48, 242001. 
(33) Sansone, G.; Kelkensberg, F.; Perez-Torres, J. F.; Morales, F.; Kling, M. F.; Siu, W.; Ghafur, O.; Johnsson, P.; Swoboda, M.; Benedetti, E.; et al., Electron Localization Following Attosecond Molecular Photoionization. Nature 2010, 465, 763-766.

(34) Cederbaum, L. S.; Zobeley, J. Ultrafast Charge Migration by Electron Correlation. Chem. Phys. Lett. 1999, 307, 205-210.

(35) Remacle, F.; Levine, R. D. An Electronic Time Scale in Chemistry. Proc. Natl. Acad. Sci. U.S.A. 2006, 103, 6793-8.

(36) Hennig, H.; Breidbach, J.; Cederbaum, L. S. Electron Correlation as the Driving Force for Charge Transfer: Charge Migration Following Ionization in N-Methyl Acetamide. J. Phys. Chem. A 2005, 109, 409-414.

(37) Breidbach, J.; Cederbaum, L. S. Universal Attosecond Response to the Removal of an Electron. Phys. Rev. Lett. 2005, 94, 033901.

(38) McPherson, A.; Gibson, G.; Jara, H.; Johann, U.; Luk, T. S.; McIntyre, I. A.; Boyer, K.; Rhodes, C. K. Studies of Multiphoton Production of Vacuum-Ultraviolet Radiation in the Rare Gases. J. Opt. Soc. Am. B 1987, 4, 595-601.

(39) Ferray, M.; L'Huillier, A.; Li, X. F.; Lompre, L. A.; Mainfray, G.; Manus, C. MultipleHarmonic Conversion of $1064 \mathrm{~nm}$ Radiation in Rare Gases. J. Phys. B: At., Mol. Opt. Phys.: At., Mol. Opt. Phys. 1988, 21, L31.

(40) Macklin, J. J.; Kmetec, J. D.; Gordon, C. L. High-Order Harmonic Generation Using Intense Femtosecond Pulses. Phys. Rev. Lett. 1993, 70, 766-769.

(41) L'Huillier, A.; Balcou, P. High-Order Harmonic Generation in Rare Gases with a 1-ps 1053-nm Laser. Phys. Rev. Lett. 1993, 70, 774-777.

(42) Krause, J. L.; Schafer, K. J.; Kulander, K. C. High-Order Harmonic Generation from Atoms and Ions in the High Intensity Regime. Phys. Rev. Lett. 1992, 68, 3535-3538. 
(43) Kulander, K. C.; Schafer, K. J.; Krause, J. L. In Super-Intense Laser-Atom Phys.; Piraux, B., L’Huillier, A., Rzazewski, K., Eds.; Plenum, New York: New York, USA, 1993; Vol. 316.

(44) Schafer, K. J.; Yang, B.; DiMauro, L. F.; Kulander, K. C. Above Threshold Ionization Beyond the High Harmonic Cutoff. Phys. Rev. Lett. 1993, 70, 1599-1602.

(45) Corkum, P. B. Plasma Perspective on Strong Field Multiphoton Ionization. Phys. Rev. Lett. 1993, 71, 1994-1997.

(46) Kuchiev, M. Y. Atomic Antenna. JETP Lett. 1987, 45, 404-406.

(47) Brunel, F. Not-so-Resonant, Resonant Absorption. Phys. Rev. Lett. 1987, 59, 52-55.

(48) Brunel, F. Harmonic Generation due to Plasma Effects in a Gas Undergoing Multiphoton Ionization in the High-Intensity Limit. J. Opt. Soc. Am. B 1990, 7, 521-526.

(49) Corkum, P. B.; Burnett, N. H.; Brunel, F. Above-Threshold Ionization in the LongWavelength Limit. Phys. Rev. Lett. 1989, 62, 1259-1262.

(50) Keldysh, L. V. Ionization in the Field of a Strong Electromagnetic Wave. Sov. Phys. JETP 1965, 20, 1307-1314.

(51) Yudin, G.; Ivanov, M. Nonadiabatic Tunnel Ionization: Looking Inside a Laser Cycle. Phys. Rev. A 2001, 64, 134091.

(52) Smirnova, O.; Ivanov, M. Multielectron High Harmonic Generation: Simple Man on a Complex Plane; 2014; pp 201-256.

(53) Perelomov, A. M.; Popov, V. S.; Terent'ev, M. V. Ionization of Atoms in an Alternating Electric Field. I. Sov. Phys. JETP 1966, 23, 924-934.

(54) Perelomov, A. M.; Popov, V. S.; Terent'ev, M. V. Ionization of Atoms in an Alternating Electric Field. II. Sov. Phys. JETP 1967, 24, 207-217. 
(55) Perelomov, A. M.; Popov, V. S.; Terent'ev, M. V. Ionization of Atoms in an Alternating Electric Field. III. Sov. Phys. JETP 1967, 25, 336-343.

(56) Ammosov, M. V.; Delone, N. B.; Krainov, V. P. Tunnel Ionization of Complex Atoms and of Atomic Ions in an Alternating Electromagnetic Field. Sov. Phys. JETP 1986, $64,1191-1194$.

(57) Chang, Z. Fundamentals of Attosecond Optics; CRC Press: Boca Raton, FL, USA, 2011.

(58) Lewenstein, M.; Balcou, P.; Ivanov, M. Y.; L'Huillier, A.; Corkum, P. B. Theory of High-Harmonic Generation by Low-Frequency Laser Fields. Phys. Rev. A 1994, 49, 2117-2132.

(59) Augst, S.; Strickland, D.; Meyerhofer, D. D.; Chin, S. L.; Eberly, J. H. Tunneling Ionization of Noble Gases in a High-Intensity Laser Field. Phys. Rev. Lett. 1989, 63, 2212-2215.

(60) Walker, B.; Sheehy, B.; DiMauro, L. F.; Agostini, P.; Schafer, K. J.; Kulander, K. C. Precision Measurement of Strong Field Double Ionization of Helium. Phys. Rev. Lett. 1994, 73, 1227-1230.

(61) Talebpour, A.; Larochelle, S.; Chin, S. L. Suppressed tunnelling ionization of the $\mathrm{D}_{2}$ molecule in an intense Ti:sapphire laser pulse. J. Phys. B: At., Mol. Opt. Phys.: At., Mol. Opt. Phys. 1998, 31, L49-L58.

(62) Lezius, M.; Blanchet, V.; Rayner, D. M.; Villeneuve, D. M.; Stolow, A.; Ivanov, M. Y. Nonadiabatic Multielectron Dynamics in Strong Field Molecular Ionization. Phys. Rev. Lett. 2001, 86, 51-54.

(63) Markevitch, A. N.; Smith, S. M.; Romanov, D. A.; Bernhard Schlegel, H.; 
Ivanov, M. Y.; Levis, R. J. Nonadiabatic Dynamics of Polyatomic Molecules and Ions in Strong Laser Fields. Phys. Rev. A 2003, 68, 011402.

(64) Salières, P.; Carré, B.; Le Déroff, L.; Grasbon, F.; Paulus, G. G.; Walther, H.; Kopold, R.; Becker, W.; Milošević, D. B.; Sanpera, A.; et al., Feynman's Path-Integral Approach for Intense-Laser-Atom Interactions. Science 2001, 292, 902-905.

(65) Sansone, G.; Vozzi, C.; Stagira, S.; Nisoli, M. Nonadiabatic Quantum Path Analysis of High-Order Harmonic Generation: Role of the Carrier-Envelope Phase on Short and Long Paths. Phys. Rev. A 2004, 70, 013411.

(66) Farkas, G.; Tóth, C. Proposal forAattosecond Light Pulse Generation Using Laser Induced Multiple-Harmonic Conversion Processes in Rare Gases. Phys. Lett. A 1992, $168,447-450$.

(67) Mairesse, Y.; de Bohan, A.; Frasinski, L. J.; Merdji, H.; Dinu, L. C.; Monchicourt, P.; Breger, P.; Kovačev, M.; Taïeb, R.; Carré, B.; et al., Attosecond Synchronization of High-Harmonic Soft X-rays. Science 2003, 302, 1540-1543.

(68) Salières, P.; L'Huillier, A.; Lewenstein, M. Coherence Control of High-Order Harmonics. Phys. Rev. Lett. 1995, 74, 3776-3779.

(69) Balcou, P.; Salières, P.; L'Huillier, A.; Lewenstein, M. Generalized Phase-Matching Conditions for High Harmonics: The Role of Field-Gradient Forces. Phys. Rev. A 1997, 55, 3204-3210.

(70) Kim, K. T.; Kim, C. M.; Baik, M.-G.; Umesh, G.; Nam, C. H. Single sub-50-Attosecond Pulse Generation from Chirp-Compensated Harmonic Radiation Using Material Dispersion. Phys. Rev. A 2004, 69, 051805.

(71) López-Martens, R.; Varjú, K.; Johnsson, P.; Mauritsson, J.; Mairesse, Y.; Salières, P.; 
Gaarde, M. B.; Schafer, K. J.; Persson, A.; Svanberg, S.; et al., Amplitude and Phase Control of Attosecond Light Pulses. Phys. Rev. Lett. 2005, 94, 033001.

(72) Frassetto, F.; Villoresi, P.; Poletto, L. Optical Concept of a Compressor for XUV Pulses in the Attosecond Domain. Opt. Express 2008, 16, 6652-6667.

(73) Poletto, L.; Villoresi, P.; Benedetti, E.; Ferrari, F.; Stagira, S.; Sansone, G.; Nisoli, M. Intense Femtosecond Extreme Ultraviolet Pulses by Using a Time-Delay-Compensated Monochromator. Opt. Lett. 2007, 32, 2897-2899.

(74) Schweigert, I. V.; Mukamel, S. Coherent Ultrafast Core-Hole Correlation Spectroscopy: X-Ray Analogues of Multidimensional NMR. Phys. Rev. Lett. 2007, 99, 163001.

(75) Seres, E.; Seres, J.; Spielmann, C. X-Ray Absorption Spectroscopy in the keV Range with Laser Generated High Harmonic Radiation. Appl. Phys. Lett. 2006, 89, 181919.

(76) Shan, B.; Chang, Z. Dramatic Extension of the High-Order Harmonic Cutoff by Using a Long-Wavelength Driving Field. Phys. Rev. A 2001, 65, 011804.

(77) Popmintchev, T.; Chen, M.-C.; Cohen, O.; Grisham, M. E.; Rocca, J. J.; Murnane, M. M.; Kapteyn, H. C. Extended Phase Matching of High Harmonics Driven by Mid-Infrared Light. Opt. Lett. 2008, 33, 2128-2130.

(78) Colosimo, P.; Doumy, G.; Blaga, C. I.; Wheeler, J.; Hauri, C.; Catoire, F.; Tate, J.; Chirla, R.; March, a. M.; Paulus, G. G.; et al., Scaling Strong-Field Interactions Towards the Classical Limit. Nat. Phys. 2008, 4, 386-389.

(79) Takahashi, E. J.; Kanai, T.; Ishikawa, K. L.; Nabekawa, Y.; Midorikawa, K. Coherent Water Window X Ray by Phase-Matched High-Order Harmonic Generation in Neutral Media. Phys. Rev. Lett. 2008, 101, 253901.

(80) Vozzi, C.; Calegari, F.; Frassetto, F.; Poletto, L.; Sansone, G.; Villoresi, P.; Nisoli, M.; 
De Silvestri, S.; Stagira, S. Coherent Continuum Generation Above 100 ev Driven by an Ir Parametric Source in a Two-Color Scheme. Phys. Rev. A 2009, 79, 033842.

(81) Shiner, A. D.; Trallero-Herrero, C.; Kajumba, N.; Bandulet, H.-C.; Comtois, D.; Légaré, F.; Giguère, M.; Kieffer, J.-C.; Corkum, P. B.; Villeneuve, D. M. Wavelength Scaling of High Harmonic Generation Efficiency. Phys. Rev. Lett. 2009, 103, 073902.

(82) Popmintchev, T.; Chen, M.-C.; Popmintchev, D.; Arpin, P.; Brown, S.; Alisauskas, S.; Andriukaitis, G.; Balciunas, T.; Mucke, O. D.; Pugzlys, A.; et al., Bright Coherent Ultrahigh Harmonics in the keV X-ray Regime from Mid-Infrared Femtosecond Lasers. Science 2012, 336, 1287-1291.

(83) Chen, M.-C.; Mancuso, C.; Hernández-García, C.; Dollar, F.; Galloway, B.; Popmintchev, D.; Huang, P.-C.; Walker, B.; Plaja, L.; Jaroń-Becker, A. A.; et al., Generation of Bright Isolated Attosecond Soft X-Ray Pulses Driven by Multicycle Midinfrared Lasers. Proc. Natl. Acad. Sci. U.S.A. 2014, 111, E2361-7.

(84) Ishii, N.; Kaneshima, K.; Kitano, K.; Kanai, T.; Watanabe, S.; Itatani, J. CarrierEnvelope Phase-Dependent High-Harmonic Generation in the Water Window Using a Few-Cycle Infrared Light Source. Nat. Commun. 2014, 5, 3331.

(85) Cousin, S. L.; Silva, F.; Teichmann, S.; Hemmer, M.; Buades, B.; Biegert, J. HighFlux Table-Top Soft X-Ray Source Driven by sub-2-Cycle, CEP Stable, $185-\mu \mathrm{m} \mathrm{1-kHz}$ Pulses for Carbon K-Edge Spectroscopy. Opt. Lett. 2014, 39, 5383-5386.

(86) Silva, F.; Teichmann, S. M.; Cousin, S. L.; Hemmer, M.; Biegert, J. Spatiotemporal Isolation of Attosecond Soft X-Ray Pulses in the Water Window. Nat. Commun. 2015, 6,6611 .

(87) Böwering, N.; Lischke, T.; Schmidtke, B.; Müller, N.; Khalil, T.; Heinzmann, U. Asymmetry in Photoelectron Emission from Chiral Molecules Induced by Circularly Polarized Light. Phys. Rev. Lett. 2001, 86, 1187-1190. 
(88) Gierz, I.; Lindroos, M.; Höchst, H.; Ast, C. R.; Kern, K. Graphene Sublattice Symmetry and Isospin Determined by Circular Dichroism in Angle-Resolved Photoemission Spectroscopy. Nano Lett. 2012, 12, 3900-3904.

(89) Schütz, G.; Knülle, M.; Ebert, H. Magnetic circular x-ray dichroism and its relation to local moments. Phys. Scr. 1993, 1993, 302.

(90) Calegari, F.; Sansone, G.; Stagira, S.; Vozzi, C.; Nisoli, M. Advances in Attosecond Science. J. Phys. B: At., Mol. Opt. Phys.: At., Mol. Opt. Phys. 2016, 49, 062001.

(91) Weihe, F. A.; Dutta, S. K.; Korn, G.; Du, D.; Bucksbaum, P. H.; Shkolnikov, P. L. Polarization of High-Intensity High-Harmonic Generation. Phys. Rev. A 1995, 51, R3433-R3436.

(92) Zhou, X.; Lock, R.; Wagner, N.; Li, W.; Kapteyn, H. C.; Murnane, M. M. Elliptically Polarized High-Order Harmonic Emission from Molecules in Linearly Polarized Laser Fields. Phys. Rev. Lett. 2009, 102, 073902.

(93) Vodungbo, B.; Barszczak Sardinha, A.; Gautier, J.; Lambert, G.; Valentin, C.; Lozano, M.; Iaquaniello, G.; Delmotte, F.; Sebban, S.; Lüning, J.; et al., Polarization Control of High Order Harmonics in the EUV Photon Energy Range. Opt. Express 2011, 19, 4346-4356.

(94) Long, S.; Becker, W.; McIver, J. K. Model Calculations of Polarization-Dependent Two-Color High-Harmonic Generation. Phys. Rev. A 1995, 52, 2262-2278.

(95) Eichmann, H.; Egbert, A.; Nolte, S.; Momma, C.; Wellegehausen, B.; Becker, W.; Long, S.; McIver, J. K. Polarization-Dependent High-Order Two-Color Mixing. Phys. Rev. A 1995, 51, R3414-R3417.

(96) Fleischer, A.; Kfir, O.; Diskin, T.; Sidorenko, P.; Cohen, O. Spin Angular Momentum 
and Tunable Polarization in High-Harmonic Generation. Nat. Photonics 2014, 8, 543549.

(97) Kfir, O.; Grychtol, P.; Turgut, E.; Knut, R.; Zusin, D.; Popmintchev, D.; Popmintchev, T.; Nembach, H.; Shaw, J. M.; Fleischer, A.; et al., Generation of Bright Phase-Matched Circularly-Polarized Extreme Ultraviolet High Harmonics. Nat. Photonics 2015, 9, 99-105.

(98) Ferré, A.; Handschin, C.; Dumergue, M.; Burgy, F.; Comby, A.; Descamps, D.; Fabre, B.; Garcia, G. a.; Géneaux, R.; Merceron, L.; et al., A Table-Top Ultrashort Light Source in the Extreme Ultraviolet for Circular Dichroism Experiments. Nat. Photonics 2015, 9, 93-98.

(99) Yuan, K.-J.; Bandrauk, A. D. Single Circularly Polarized Attosecond Pulse Generation by Intense Few Cycle Elliptically Polarized Laser Pulses and Terahertz Fields from Molecular Media. Phys. Rev. Lett. 2013, 110, 023003.

(100) Yuan, K.-J.; Bandrauk, A. D. Attosecond-Magnetic-Field-Pulse Generation by Intense Few-Cycle Circularly Polarized UV Laser Pulses. Phys. Rev. A 2013, 88, 013417.

(101) Reichert, J.; Holzwarth, R.; Udem, T.; Hänsch, T. W. Measuring the Frequency of Light with Mode-Locked Lasers. Opt. Commun. 1999, 172, 59-68.

(102) Telle, H. R.; Steinmeyer, G.; Dunlop, A. E.; Stenger, J.; Sutter, D.; Keller, U. CarrierEnvelope Offset Phase Control: A Novel Concept for Absolute Optical Frequency Measurement and Ultrashort Pulse Generation. Appl. Phys. B 1999, 69, 327-332.

(103) Apolonski, A.; Poppe, A.; Tempea, G.; Spielmann, C.; Udem, T.; Holzwarth, R.; Hänsch, T. W.; Krausz, F. Controlling the Phase Evolution of Few-Cycle Light Pulses. Phys. Rev. Lett. 2000, 85, 740-743. 
(104) Jones, D. J.; Diddams, S. A.; Ranka, J. K.; Stentz, A.; Windeler, R. S.; Hall, J. L.; Cundiff, S. T. Carrier-Envelope Phase Control of Femtosecond Mode-Locked Lasers and Direct Optical Frequency Synthesis. Science 2000, 288, 635-639.

(105) Strickland, D; Mourou, G. Compression Of Amplified Chirped Optical Pulses. Opt. Commun. 1985, 56, 219-221.

(106) Baltuška, A.; Udem, T.; Uiberacker, M.; Hentschel, M.; Goulielmakis, E.; Gohle, C.; Holzwarth, R.; Yakovlev, V. S.; Scrinzi, A.; Hänsch, T. W.; et al., Attosecond Control of Electronic Processes by Intense Light Fields. Nature 2003, 421, 611-615.

(107) Baltuška, A.; Fuji, T.; Kobayashi, T. Controlling the Carrier-Envelope Phase of Ultrashort Light Pulses with Optical Parametric Amplifiers. Phys. Rev. Lett. 2002, 88, 133901.

(108) Paulus, G. G.; Grasbon, F.; Walther, H.; Villoresi, P.; Nisoli, M.; Stagira, S.; Priori, E.; De Silvestri, S. Absolute-Phase Phenomena in Photoionization with Few-Cycle Laser Pulses. Nature 2001, 414, 182-184.

(109) Dubietis, A.; Jonušauskas, G.; Piskarskas, A. Powerful Femtosecond Pulse Generation by Chirped and Stretched Pulse Parametric Amplification in BBO Crystal. Opt. Commun. 1992, 88, 437-440.

(110) Fattahi, H.; Barros, H. G.; Gorjan, M.; Nubbemeyer, T.; Alsaif, B.; Teisset, C. Y.; Schultze, M.; Prinz, S.; Haefner, M.; Ueffing, M.; et al., Third-Generation Femtosecond Technology. Optica 2014, 1, 45-63.

(111) Nisoli, M.; De Silvestri, S.; Svelto, O. Generation of High-Energy 10-fs Pulses by a New Pulse Compression Technique. Appl. Phys. Lett. 1996, 68, 2793-2795.

(112) Nisoli, M.; De Silvestri, S.; Svelto, O.; Szipöcs, R.; Ferencz, K.; Spielmann, C.; Sar- 
tania, S.; Krausz, F. Compression of High-Energy Laser Pulses Below 5 fs. Opt. Lett. 1997, 22, 522-524.

(113) Nisoli, M.; Stagira, S.; De Silvestri, S.; Svelto, O.; Sartania, S.; Cheng, Z.; Tempea, G.; Spielmann, C.; Krausz, F. Toward a Terawatt-Scale sub-10-fs Laser Technology. J. Sel. Top. Quantum Electron 1998, 4, 414-419.

(114) Dutin, C. F.; Dubrouil, A.; Petit, S.; Mével, E.; Constant, E.; Descamps, D. PostCompression of High-Energy Femtosecond Pulses Using Gas Ionization. Opt. Lett. 2010, 35, 253-255.

(115) Südmeyer, T.; Marchese, S. V.; Hashimoto, S.; Baer, C. R. E.; Gingras, G.; Witzel, B.; Keller, U. Femtosecond Laser Oscillators for High-Field Science. Nat. Photonics 2008, 2, 599-604.

(116) Jones, R. J.; Moll, K. D.; Thorpe, M. J.; Ye, J. Phase-Coherent Frequency Combs in the Vacuum Ultraviolet via High-Harmonic Generation inside a Femtosecond Enhancement Cavity. Phys. Rev. Lett. 2005, 94, 193201.

(117) Gohle, C.; Udem, T.; Herrmann, M.; Rauschenberger, J.; Holzwarth, R.; Schuessler, H. a.; Krausz, F.; Hänsch, T. W. A Frequency Comb in the Extreme Ultraviolet. Nature 2005, 436, 234-237.

(118) Ozawa, A.; Rauschenberger, J.; Gohle, C.; Herrmann, M.; Walker, D. R.; Pervak, V.; Fernandez, A.; Graf, R.; Apolonski, A.; Holzwarth, R.; et al., High Harmonic Frequency Combs for High Resolution Spectroscopy. Phys. Rev. Lett. 2008, 100, 253901.

(119) Cingoz, A.; Yost, D. C.; Allison, T. K.; Ruehl, A.; Fermann, M. E.; Hartl, I.; Ye, J. Direct Frequency Comb Spectroscopy in the Extreme Ultraviolet. Nature 2011, 482, 10711. 
(120) Eidam, T.; Hanf, S.; Seise, E.; Andersen, T. V.; Gabler, T.; Wirth, C.; Schreiber, T.; Limpert, J.; Tünnermann, A. Femtosecond Fiber CPA System Emitting 830 W Average Output Power. Opt. Lett. 2010, 35, 94-96.

(121) Hädrich, S.; Klenke, A.; Rothhardt, J. High Photon Flux Table-Top Coherent Extreme Ultraviolet Source. Nat. Photonics 2014, 8, 779-783.

(122) Rothhardt, J.; Demmler, S.; Hädrich, S.; Limpert, J.; Tünnermann, A. OctaveSpanning OPCPA System Delivering CEP-Stable Few-Cycle Pulses and 22 W of Average Power at $1 \mathrm{MHz}$ Repetition Rate. Opt. Express 2012, 20, 10870-10878.

(123) Krebs, M.; Hädrich, S.; Demmler, S.; Rothhardt, J.; Zaïr, A.; Chipperfield, L.; Limpert, J.; Tünnermann, A. Towards Isolated Attosecond Pulses at Megahertz Repetition Rates. Nat. Photonics 2013, 7, 555-559.

(124) Chini, M.; Zhao, K.; Chang, Z. The Generation, Characterization and Applications of Broadband Isolated Attosecond Pulses. Nat. Photonics 2014, 8, 178-186.

(125) Nisoli, M.; Sansone, G. New Frontiers in Attosecond Science. Prog. Quantum Electron. 2009, 33, $17-59$.

(126) Sansone, G.; Poletto, L.; Nisoli, M. High-Energy Attosecond Light Sources. Nat. Photonics 2011, 5, 655-663.

(127) Kienberger, R.; Goulielmakis, E.; Uiberacker, M.; Baltuška, A.; Yakovlev, V.; Bammer, F.; Scrinzi, A.; Westerwalbesloh, T.; Kleineberg, U.; Heinzmann, U.; et al., Atomic Transient Recorder. Nature 2004, 427, 817-821.

(128) Christov, I. P.; Murnane, M. M.; Kapteyn, H. C. High-Harmonic Generation of Attosecond Pulses in the "Single-Cycle" Regime. Phys. Rev. Lett. 1997, 78, 1251-1254.

(129) Burnett, N. H.; Kan, C.; Corkum, P. B. Ellipticity and Polarization Effects in Harmonic Generation in Ionizing Neon. Phys. Rev. A 1995, 51, R3418-R3421. 
(130) Corkum, P. B.; Burnett, N. H.; Ivanov, M. Y. Subfemtosecond Pulses. Opt. Lett. 1994, 19, 1870-1872.

(131) Tcherbakoff, O.; Mével, E.; Descamps, D.; Plumridge, J.; Constant, E. Time-Gated High-Order Harmonic Generation. Phys. Rev. A 2003, 68, 043804.

(132) Sola, I.; Mével, E.; Elouga, L.; Constant, E.; Strelkov, V.; Poletto, L.; Villoresi, P.; Benedetti, E.; Caumes, J. P.; Stagira, S.; et al., Controlling Attosecond Electron Dynamics by Phase-Stabilized Polarization Gating. Nat. Phys. 2006, 2, 319-322.

(133) Sansone, G.; Benedetti, E.; Calegari, F.; Vozzi, C.; Avaldi, L.; Flammini, R.; Poletto, L.; Villoresi, P.; Altucci, C.; Velotta, R.; et al., Isolated Single-Cycle Attosecond Pulses. Science 2006, 314, 443-446.

(134) Chang, Z. Controlling Attosecond Pulse Generation with a Double Optical Gating. Phys. Rev. A 2007, 76, 051403.

(135) Mashiko, H.; Gilbertson, S.; Li, C.; Khan, S. D.; Shakya, M. M.; Moon, E.; Chang, Z. Double Optical Gating of High-Order Harmonic Generation with Carrier-Envelope Phase Stabilized Lasers. Phys. Rev. Lett. 2008, 100, 103906.

(136) Mashiko, H.; Gilbertson, S.; Chini, M.; Feng, X.; Yun, C.; Wang, H.; Khan, S. D.; Chen, S.; Chang, Z. Extreme Ultraviolet Supercontinua Supporting Pulse Durations of Less than One Atomic Unit of Time. Opt. Lett. 2009, 34, 3337-3339.

(137) Mashiko, H.; Bell, M. J.; Beck, A. R.; Abel, M. J.; Nagel, P. M.; Steiner, C. P.; Robinson, J.; Neumark, D. M.; Leone, S. R. Tunable Frequency-Controlled Isolated Attosecond Pulses Characterized by either $750 \mathrm{~nm}$ or $400 \mathrm{~nm}$ Wavelength Streak Fields. Opt. Express 2010, 18, 25887-25895.

(138) Feng, X.; Gilbertson, S.; Mashiko, H.; Wang, H.; Khan, S. D.; Chini, M.; Wu, Y.; 
Zhao, K.; Chang, Z. Generation of Isolated Attosecond Pulses with 20 to 28 Femtosecond Lasers. Phys. Rev. Lett. 2009, 103, 183901.

(139) Abel, M. J.; Pfeifer, T.; Nagel, P. M.; Boutu, W.; Bell, M. J.; Steiner, C. P.; Neumark, D. M.; Leone, S. R. Isolated Attosecond Pulses From Ionization Gating of High-Harmonic Emission. Chem. Phys. 2009, 366, 9-14, Attosecond Molecular Dynamics.

(140) Thomann, I.; Bahabad, A.; Liu, X.; Trebino, R.; Murnane, M. M.; Kapteyn, H. C. Characterizing Isolated Attosecond Pulses from Hollow-Core Waveguides Using MultiCycle Driving Pulses. Opt. Express 2009, 17, 4611-4633.

(141) Ferrari, F.; Calegari, F.; Lucchini, M.; Vozzi, C.; Stagira, S.; Sansone, G.; Nisoli, M. High-Energy Isolated Attosecond Pulses Generated by Above-Saturation Few-Cycle Fields. Nat. Photonics 2010, 4, 875-879.

(142) Vincenti, H.; Quéré, F. Attosecond Lighthouses: How To Use Spatiotemporally Coupled Light Fields To Generate Isolated Attosecond Pulses. Phys. Rev. Lett. 2012, 108, 113904.

(143) Wheeler, J. A.; Borot, A.; Monchocé, S.; Vincenti, H.; Ricci, A.; Malvache, A.; LopezMartens, R.; Quéré, F. Attosecond Lighthouses from Plasma Mirrors. Nat. Photonics 2012, 6, 829-833.

(144) Kim, K. T.; Zhang, C.; Ruchon, T.; Hergott, J. F.; Auguste, T.; Villeneuve, D.; Corkum, P. B.; Quéré, F. Photonic Streaking of Attosecond Pulse Trains. Nat. Photonics 2013, 7, 651-656.

(145) Hammond, T.; Brown, G.; Kim, K.; Villeneuve, D.; Corkum, P. Attosecond Pulses Measured from the Attosecond Lighthouse. Nat. Photonics 2016, 10, 171-175. 
(146) Louisy, M.; Arnold, C. L.; Miranda, M.; Larsen, E. W.; Bengtsson, S. N.; Kroon, D.; Kotur, M.; Guénot, D.; Rading, L.; Rudawski, P.; et al., Gating Attosecond Pulses in a Noncollinear Geometry. Optica 2015, 2, 563-566.

(147) Wirth, A.; Hassan, M. T.; Grguraš, I.; Gagnon, J.; Moulet, A.; Luu, T. T.; Pabst, S.; Santra, R.; Alahmed, Z. A.; Azzeer, A. M.; et al., Synthesized Light Transients. Science 2011, 334, 195-200.

(148) Hassan, M. T.; Wirth, A.; Grguraš, I.; Moulet, A.; Luu, T. T.; Gagnon, J.; Pervak, V.; Goulielmakis, E. Attosecond Photonics: Synthesis and Control of Light Transients. Rev. Sci. Instrum. 2012, 83, 111301.

(149) Hassan, M. T.; Luu, T. T.; Moulet, A.; Raskazovskaya, O.; Zhokhov, P.; Garg, M.; Karpowicz, N.; Zheltikov, A. M.; Pervak, V.; Krausz, F.; et al., Optical Attosecond Pulses and Tracking the Nonlinear Response of Bound Electrons. Nature 2016, 530, $66-70$.

(150) Véniard, V.; Taïeb, R.; Maquet, A. Two-Color Multiphoton Ionization of Atoms Using High-Order Harmonic Radiation. Phys. Rev. Lett. 1995, 74, 4161-4164.

(151) Véniard, V.; Taïeb, R.; Maquet, A. Phase Dependence of $(\mathrm{N}+1)$-Color $(\mathrm{N}>1) \operatorname{Ir}-\mathrm{Uv}$ Photoionization of Atoms with Higher Harmonics. Phys. Rev. A 1996, 54, 721-728.

(152) Wigner, E. P. Lower Limit for the Energy Derivative of the Scattering Phase Shift. Phys. Rev. 1955, 98, 145-147.

(153) Jiménez-Galán, A.; Argenti, L.; Martín, F. Modulation of Attosecond Beating in Resonant Two-Photon Ionization. Phys. Rev. Lett. 2014, 113, 263001.

(154) Pazourek, R.; Nagele, S.; Burgdörfer, J. Attosecond Chronoscopy of Photoemission. Rev. Mod. Phys. 2015, 87, 765-802. 
(155) Kotur, M.; Guénot, D.; Jiménez-Galán, A.; Kroon, D.; Larsen, E. W.; Louisy, M.; Bengtsson, S.; Miranda, M.; Mauritsson, J.; Arnold, C. L.; et al., Spectral Phase Measurement of a Fano Resonance Using Tunable Attosecond Pulses. Nat. Commun. 2016, $7,10566$.

(156) Haessler, S.; Fabre, B.; Higuet, J.; Caillat, J.; Ruchon, T.; Breger, P.; Carré, B.; Constant, E.; Maquet, A.; Mével, E.; et al., Phase-Resolved Attosecond Near-Threshold Photoionization of Molecular Nitrogen. Phys. Rev. A 2009, 80, 011404.

(157) Huppert, M.; Jordan, I.; Baykusheva, D.; von Conta, A.; Wörner, H. J. Attosecond Delays in Molecular Photoionization. Phys. Rev. Lett. 2016, 117, 093001.

(158) Kitzler, M.; Milosevic, N.; Scrinzi, A.; Krausz, F.; Brabec, T. Quantum Theory of Attosecond XUV Pulse Measurement by Laser Dressed Photoionization. Phys. Rev. Lett. 2002, 88, 173904.

(159) Itatani, J.; Quéré, F.; Yudin, G. L.; Ivanov, M. Y.; Krausz, F.; Corkum, P. B. Attosecond Streak Camera. Phys. Rev. Lett. 2002, 88, 173903.

(160) Mairesse, Y.; Quéré, F. Frequency-Resolved Optical Gating for Complete Reconstruction of Attosecond Bursts. Phys. Rev. A 2005, 71, 011401.

(161) Kane, D. J.; Trebino, R. Characterization of Arbitrary Femtosecond Pulses Using Frequency-Resolved Optical Gating. IEEE J. Quantum Electron. 1993, 29, 571-579.

(162) Kane, D. J. Recent Progress Toward Real-Time Measurement of Ultrashort Laser Pulses. IEEE J. Quantum Electron. 1999, 35, 421-431.

(163) Goulielmakis, E.; Uiberacker, M.; Kienberger, R.; Baltuska, A.; Yakovlev, V.; Scrinzi, A.; Westerwalbesloh, T.; Kleineberg, U.; Heinzmann, U.; Drescher, M.; et al., Direct Measurement of Light Waves. Science 2004, 305, 1267-1269. 
(164) Goulielmakis, E.; Schultze, M.; Hofstetter, M.; Yakovlev, V. S.; Gagnon, J.; Uiberacker, M.; Aquila, A. L.; Gullikson, E. M.; Attwood, D. T.; Kienberger, R.; et al., Single-Cycle Nonlinear Optics. Science 2008, 320, 1614-1617.

(165) Feng, X.; Gilbertson, S.; Mashiko, H.; Wang, H.; Khan, S. D.; Chini, M.; Wu, Y.; Zhao, K.; Chang, Z. Generation of Isolated Attosecond Pulses with 20 to 28 Femtosecond Lasers. Phys. Rev. Lett. 2009, 103, 183901.

(166) Gilbertson, S.; Wu, Y.; Khan, S. D.; Chini, M.; Zhao, K.; Feng, X.; Chang, Z. Isolated Attosecond Pulse Generation Using Multicycle Pulses Directly from a Laser Amplifier. Phys. Rev. A 2010, 81, 043810.

(167) Chini, M.; Gilbertson, S.; Khan, S. D.; Chang, Z. Characterizing Ultrabroadband Attosecond Lasers. Opt. Express 2010, 18, 13006-13016.

(168) Zhao, K.; Zhang, Q.; Chini, M.; Wu, Y.; Wang, X.; Chang, Z. Tailoring a 67 Attosecond Pulse Through Advantageous Phase-Mismatch. Opt. Lett. 2012, 37, 3891-3893.

(169) Kim, K. T.; Villeneuve, D. M.; Corkum, P. B. Manipulating Quantum Paths for Novel Attosecond Measurement Methods. Nat. Photonics 2014, 8, 187-194.

(170) Kim, K. T.; Zhang, C.; Shiner, A. D.; Kirkwood, S. E.; Frumker, E.; Gariepy, G.; Naumov, A.; Villeneuve, D. M.; Corkum, P. B. Manipulation of Quantum Paths for Space-Time Characterization of Attosecond Pulses. Nat. Phys. 2013, 9, 159-163.

(171) Kim, K. T.; Zhang, C.; Shiner, A. D.; Schmidt, B. E.; Legare, F.; Villeneuve, D.; Corkum, P. B. Petahertz Optical Oscilloscope. Nat. Photonics 2013, 7, 958-962.

(172) Uehara, Y.; Ushiroku, T.; Ushioda, S.; Murata, Y. High Resolution Time-of-Flight Electron Spectrometer. Jpn. J. Appl. Phys. 1990, 29, 2858-2863.

(173) Wiley, W. C.; McLaren, I. H. Time of Flight Mass Spectrometer with Improved Resolution. Rev. Sci. Instrum. 1955, 26, 1150-1157. 
(174) Kruit, P.; Read, F. H. Magnetic Field Paralleliser for $2 \pi$ Electron-Spectrometer and Electron-Image Magnifier. J. Phys. E: Sci. Instrum. 1983, 16, 313-324.

(175) Eppink, A. T. J. B.; Parker, D. H. Velocity Map Imaging of Ions and Electrons Using Electrostatic Lenses: Application in Photoelectron and Photofragment Ion Imaging of Molecular Oxygen. Rev. Sci. Instrum. 1997, 68, 3477-3484.

(176) Vrakking, M. J. J. An Iterative Procedure for the Inversion of Two-Dimensional Ion/Photoelectron Imaging Experiments. Rev. Sci. Instrum. 2001, 72, 4084-4089.

(177) Trabattoni, A.; Klinker, M.; González-Vázquez, J.; Liu, C.; Sansone, G.; Linguerri, R.; Hochlaf, M.; Klei, J.; Vrakking, M. J. J.; Martín, F.; et al., Mapping the Dissociative Ionization Dynamics of Molecular Nitrogen with Attosecond Time Resolution. Phys. Rev. X 2015, 5, 041053.

(178) Neidel, C.; Klei, J.; Yang, C.-H.; Rouzée, A.; Vrakking, M. J. J.; Klünder, K.; Miranda, M.; Arnold, C. L.; Fordell, T.; L'Huillier, A.; et al., Probing Time-Dependent Molecular Dipoles on the Attosecond Time Scale. Phys. Rev. Lett. 2013, 111, 033001.

(179) Ghafur, O.; Siu, W.; Johnsson, P.; Kling, M. F.; Drescher, M.; Vrakking, M. J. J. A Velocity Map Imaging Detector with an Integrated Gas Injection System. Rev. Sci. Instrum. 2009, 80, 033110.

(180) Ullrich, J.; Moshammer, R.; Dorn, A.; D Rner, R.; Schmidt, L. P. H.; Schmidt-B Cking, H. Recoil-Ion and Electron Momentum Spectroscopy: Reaction-Microscopes. Rep. Prog. Phys. 2003, 66, 1463-1545.

(181) Dörner, R.; Mergel, V.; Jagutzki, O.; Spielberger, L.; Ullrich, J.; Moshammer, R.; Schmidt-Böcking, H. Cold Target Recoil Ion Momentum Spectroscopy: a 'Momentum Microscope' to View Atomic Collision Dynamics. Phys. Rep. 2000, 330, 95 - 192. 
(182) Ullrich, J.; Moshammer, R.; Dörner, R.; Jagutzki, O.; Mergel, V.; SchmidtBöcking, H.; Spielberger, L. Recoil-ion momentum spectroscopy. J. Phys. B: At., Mol. Opt. Phys.: At., Mol. Opt. Phys. 1997, 30, 2917-2974.

(183) Sabbar, M.; Heuser, S.; Boge, R.; Lucchini, M.; Gallmann, L.; Cirelli, C.; Keller, U. Combining Attosecond XUV Pulses with Coincidence Spectroscopy. Rev. Sci. Instrum. 2014, 85, 103113.

(184) Zuo, T.; Bandrauk, A.; Corkum, P. Laser-Induced Electron Diffraction: A New Tool for Probing Ultrafast Molecular Dynamics. Chem. Phys. Lett. 1996, 259, 313-320.

(185) Li, W.; Zhou, X.; Lock, R.; Patchkovskii, S.; Stolow, A.; Kapteyn, H. C.; Murnane, M. M. Time-Resolved Dynamics in $\mathrm{N}_{2} \mathrm{O}_{4}$ Probed Using High Harmonic Generation. Science 2008, 322, 1207-1211.

(186) Yoshii, K.; Miyaji, G.; Miyazaki, K. Dynamic Properties of Angle-Dependent HighOrder Harmonic Generation from Coherently Rotating Molecules. Phys. Rev. Lett. 2008, 101, 183902.

(187) Frumker, E.; Hebeisen, C. T.; Kajumba, N.; Bertrand, J. B.; Wörner, H. J.; Spanner, M.; Villeneuve, D. M.; Naumov, A.; Corkum, P. B. Oriented Rotational WavePacket Dynamics Studies via High Harmonic Generation. Phys. Rev. Lett. 2012, 109, 113901.

(188) Kraus, P. M.; Mignolet, B.; Baykusheva, D.; Rupenyan, A.; Horný, L.; Penka, E. F.; Grassi, G.; Tolstikhin, O. I.; Schneider, J.; Jensen, F.; et al., Measurement and Laser Control of Attosecond Charge Migration in Ionized Iodoacetylene. Science 2015, 350, 790-795.

(189) Niikura, H.; Légaré, F.; Hasbani, R.; Bandrauk, a. D.; Ivanov, M. Y.; Villeneuve, D. M.; Corkum, P. B. Sub-Laser-Cycle Electron Pulses for Probing Molecular Dynamics. Nature 2002, 417, 917-922. 
(190) Niikura, H.; Villeneuve, D. M.; Corkum, P. B. Controlling Vibrational Wave Packets with Intense, Few-Cycle Laser Pulses. Phys. Rev. A 2006, 73, 021402(R).

(191) Baker, S.; Robinson, J. S.; Haworth, C. A.; Teng, H.; Smith, R. A.; Chirilă, C. C.; Lein, M.; Tisch, J. W. G.; Marangos, J. P. Probing Proton Dynamics in Molecules on an Attosecond Time Scale. Science 2006, 312, 424-427.

(192) Kreibich, T.; Lein, M.; Engel, V.; Gross, E. K. U. Even-Harmonic Generation due to Beyond-Born-Oppenheimer Dynamics. Phys. Rev. Lett. 2001, 87, 103901.

(193) Bredtmann, T.; Chelkowski, S.; Bandrauk, A. D. Effect of Nuclear Motion on Molecular High Order Harmonic Pump Probe Spectroscopy. J. Phys. Chem. A 2012, 116, 11398-11405.

(194) Silva, R. E. F.; Rivière, P.; Morales, F.; Smirnova, O.; Ivanov, M.; Martín, F. Even Harmonic Generation in Isotropic Media of Dissociating Homonuclear Molecules. Sci. Rep. 2016, 6, 32653.

(195) Serbinenko, V.; Smirnova, O. Multidimensional high harmonic spectroscopy: a semiclassical perspective on measuring multielectron rearrangement upon ionization. $J$. Phys. B: At., Mol. Opt. Phys.: At., Mol. Opt. Phys. 2013, 46, 171001.

(196) Bruner, B. D.; Soifer, H.; Shafir, D.; Serbinenko, V.; Smirnova, O.; Dudovich, N. Multidimensional High Harmonic Spectroscopy. J. Phys. B: At., Mol. Opt. Phys.: At., Mol. Opt. Phys. 2015, 48, 174006.

(197) Shafir, D.; Soifer, H.; Bruner, B. D.; Dagan, M.; Mairesse, Y.; Patchkovskii, S.; Ivanov, M. Y.; Smirnova, O.; Dudovich, N. Resolving the Time when an Electron Exits a Tunnelling Barrier. Nature 2012, 485, 343-346.

(198) Hergott, J.-F.; Kovacev, M.; Merdji, H.; Hubert, C.; Mairesse, Y.; Jean, E.; Breger, P.; 
Agostini, P.; Carré, B.; Salières, P. Extreme-Ultraviolet High-Order Harmonic Pulses in the Microjoule Range. Phys. Rev. A 2002, 66, 021801.

(199) Tzallas, P.; Charalambidis, D.; Papadogiannis, N. A.; Witte, K.; Tsakiris, G. D. Direct Observation of Attosecond Light Bunching. Nature 2003, 426, 267-271.

(200) Takahashi, E.; Nabekawa, Y.; Midorikawa, K. Generation of 10-pJ Coherent ExtremeUltraviolet Light by Use of High-Order Harmonics. Opt. Lett. 2002, 27, 1920-1922.

(201) Popmintchev, T.; Chen, M.-C.; Arpin, P.; Murnane, M. M.; Kapteyn, H. C. The Attosecond Nonlinear Optics of Bright Coherent X-Ray Generation. Nat. Photonics 2010, 4, 822-832.

(202) Rivas, D. E.; Weidman, M.; Bergues, B.; Muschet, A.; Guggenmos, A.; Razskazovskaya, O.; Schröder, H.; Helml, W.; Marcus, G.; Kienberger, R.; et al., Generation of High-Energy Isolated Attosecond Pulses for XUV-pump/XUV-probe Experiments at $100 \mathrm{eV}$. High-Brightness Sources and Light-Driven Interactions 2016, HT1B.1.

(203) Nabekawa, Y.; Hasegawa, H.; Takahashi, E. J.; Midorikawa, K. Production of Doubly Charged Helium Ions by Two-Photon Absorption of an Intense Sub-10-fs Soft X-Ray Pulse at $42 \mathrm{eV}$ Photon Energy. Phys. Rev. Lett. 2005, 94, 043001.

(204) Nabekawa, Y.; Shimizu, T.; Okino, T.; Furusawa, K.; Hasegawa, H.; Yamanouchi, K.; Midorikawa, K. Conclusive Evidence of an Attosecond Pulse Train Observed with the Mode-Resolved Autocorrelation Technique. Phys. Rev. Lett. 2006, 96, 083901.

(205) Nabekawa, Y.; Shimizu, T.; Okino, T.; Furusawa, K.; Hasegawa, H.; Yamanouchi, K.; Midorikawa, K. Interferometric Autocorrelation of an Attosecond Pulse Train in the Single-Cycle Regime. Phys. Rev. Lett. 2006, 97, 153904.

(206) Tzallas, P.; Skantzakis, E.; Kalpouzos, C.; Benis, E. P.; Tsakiris, G. D.; Charalam- 
bidis, D. Generation of Intense Continuum Extreme-Ultraviolet Radiation by ManyCycle Laser Fields. Nat. Phys. 2007, 3, 846-850.

(207) Tzallas, P.; Skantzakis, E.; Nikolopoulos, L. A. A.; Tsakiris, G. D.; Charalambidis, D. Extreme-Ultraviolet Pump-Probe Studies of One-Femtosecond-Scale Electron Dynamics. Nat. Phys. 2011, 7, 781-784.

(208) Skantzakis, E.; Tzallas, P.; Kruse, J. E.; Kalpouzos, C.; Faucher, O.; Tsakiris, G. D.; Charalambidis, D. Tracking Autoionizing-Wave-Packet Dynamics at the 1 -fs Temporal Scale. Phys. Rev. Lett. 2010, 105, 043902.

(209) Tzallas, P.; Skantzakis, E.; Charalambidis, D. Direct two-XUV-photon double ionization in xenon. J. Phys. B: At., Mol. Opt. Phys.: At., Mol. Opt. Phys. 2012, 45, 074007.

(210) Carpeggiani, P. A.; Tzallas, P.; Palacios, A.; Gray, D.; Martín, F.; Charalambidis, D. Disclosing Intrinsic Molecular Dynamics on the 1-fs Scale Through ExtremeUltraviolet Pump-Probe Measurements. Phys. Rev. A 2014, 89, 023420.

(211) Brühwiler, P. A.; Karis, O.; Mårtensson, N. Charge-Transfer Dynamics Studied using Resonant Core Spectroscopies. Rev. Mod. Phys. 2002, 74, 703-740.

(212) Coville, M.; Thomas, T. D. Molecular Effects on Inner-Shell Lifetimes: Possible Test of the One-Center Model of Auger Decay. Phys. Rev. A 1991, 43, 6053-6056.

(213) Keller, C.; Stichler, M.; Comelli, G.; Esch, F.; Lizzit, S.; Menzel, D.; Wurth, W. Femtosecond Dynamics of Adsorbate Charge-Transfer Processes as Probed by HighResolution Core-Level Spectroscopy. Phys. Rev. B 1998, 57, 11951-11954.

(214) Föhlisch, A.; Menzel, D.; Feulner, P.; Ecker, M.; Weimar, R.; Kostov, K.; Tyuliev, G.; Lizzit, S.; Larciprete, R.; Hennies, F.; et al., Energy Dependence of Resonant Charge Transfer from Adsorbates to Metal Substrates. Chem. Phys. 2003, 289, 107-115. 
(215) Chen, C. T.; DiDio, R. A.; Ford, W. K.; Plummer, E. W.; Eberhardt, W. Dynamics of Adsorbate Core-Hole Decay. Phys. Rev. B 1985, 32, 8434-8437.

(216) Wurth, W.; Schneider, C.; Treichler, R.; Umbach, E.; Menzel, D. Evolution of Adsorbate Core-Hole States After Bound and Continuum Primary Excitation: Relaxation Versus Decay. Phys. Rev. B 1987, 35, 7741-7744.

(217) Björneholm, O.; Sandell, A.; Nilsson, A.; Mårtensson, N.; Andersen, J. N. Autoionization of Adsorbates. Phys. Scr. 1992, T41, 217-225.

(218) Björneholm, O.; Nilsson, A.; Sandell, A.; Hernnäs, B.; Mårtensson, N. Determination of Time Scales for Charge-Transfer Screening in Physisorbed Molecules. Phys. Rev. Lett. 1992, 68, 1892-1895.

(219) Wurth, W.; Menzel, D. Ultrafast Electron Dynamics at Surfaces Probed by Resonant Auger Spectroscopy. Chem. Phys. 2000, 251, $141-149$.

(220) Ohno, M. Deexcitation Processes in Adsorbates. Phys. Rev. B 1994, 50, 2566-2575.

(221) Föhlisch, a.; Feulner, P.; Hennies, F.; Fink, a.; Menzel, D.; Sanchez-Portal, D.; Echenique, P. M.; Wurth, W. Direct Observation of Electron Dynamics in the Attosecond Domain. Nature 2005, 436, 373-376.

(222) Karis, O.; Nilsson, A.; Weinelt, M.; Wiell, T.; Puglia, C.; Wassdahl, N.; Mårtensson, N.; Samant, M.; Stöhr, J. One-Step and Two-Step Description of Deexcitation Processes in Weakly Interacting Systems. Phys. Rev. Lett. 1996, 76, 1380-1383.

(223) Eckle, P.; Smolarski, M.; Schlup, P.; Biegert, J.; Staudte, A.; Schöffler, M.; Muller, H. G.; Dörner, R.; Keller, U. Attosecond Angular Streaking. Nat. Phys. 2008, 4, $565-570$.

(224) Pfeiffer, A. N.; Cirelli, C.; Smolarski, M.; Dörner, R.; Keller, U. Timing the Release in Sequential Double Ionization. Nat. Phys. 2011, 7, 428-433. 
(225) Dietrich, P.; Krausz, F.; Corkum, P. B. Determining the Absolute Carrier Phase of a Few-Cycle Laser Pulse. Opt. Lett. 2000, 25, 16-18.

(226) Cirelli, C.; Pfeiffer, A. N.; Smolarski, M.; Eckle, P.; Keller, U. In Attosecond Physics; Plaja, L., Torres, R., Zair, A., Eds.; Springer-Verlag Berlin Heidelberg: New York, USA, 2013; Vol. 177.

(227) Eckle, P.; Pfeiffer, A. N.; Cirelli, C.; Staudte, A.; Dörner, R.; Muller, H. G.; Büttiker, M.; Keller, U. Attosecond Ionization and Tunneling Delay Time Measurements in Helium. Science 2008, 322, 1525-1529.

(228) Weger, M.; Maurer, J.; Ludwig, A.; Gallmann, L.; Keller, U. Transferring the Attoclock Technique to Velocity Map Imaging. Opt. Express 2013, 21, 21981-21990.

(229) Pellegrini, C.; Marinelli, A.; Reiche, S. The Physics of X-Ray Free-Electron Lasers. Rev. Mod. Phys. 2016, 88, 015006.

(230) McNeil, B. W. J.; Thompson, N. R. X-Ray Free-Electron Lasers. Nat. Photonics 2010, 4, 814-821.

(231) Bonifacio, R.; De Salvo, L.; Pierini, P.; Piovella, N.; Pellegrini, C. Spectrum, Temporal Structure, and Fluctuations in a High-Gain Free-Electron Laser Starting from Noise. Phys. Rev. Lett. 1994, 73, 70-73.

(232) Bonifacio, R.; Pellegrini, C.; Narducci, L. Collective Instabilities and High-Gain Regime in a Free Electron Laser. Opt. Commun. 1984, 50, 373 - 378.

(233) Emma, P.; Akre, R.; Arthur, J.; Bionta, R.; Bostedt, C.; Bozek1, J.; Brachmann, A.; Bucksbaum, P.; Coffee, R.; Decker, F.-J.; et al., First Lasing and Operation of an Angstrom-Wavelength Free-Electron Laser. Nat. Photonics 2010, 4, 641-647.

(234) Ackermann, W.; Asova, G.; Ayvazyan, V.; Azima, A.; Baboi, N.; Bahr, J.; Balandin, V.; Beutner, B.; Brandt, A.; Bolzmann, A.; et al., Operation of a Free-Electron 
Laser from the Extreme Ultraviolet to the Water Window. Nat. Photonics 2007, 1, $336-342$.

(235) Giannessi, L.; Alesini, D.; Biagini, M.; Boscolo, M.; Bougeard, M.; Breger, P.; Carré, B.; Castellano, M.; Cianchi, A.; Ciocci, F.; et al., Seeding Experiments at SPARC. Nucl. Instr. Meth. Phys. Res. A 2008, 593, $132-136$.

(236) Lambert, G.; Hara, T.; Garzella, D.; Tanikawa, T.; Labat, M.; Carre, B.; Kitamura, H.; Shintake, T.; Bougeard, M.; Inoue, S.; et al., Injection of Harmonics Generated in Gas in a Free-Electron Laser Providing Intense and Coherent Extreme-Ultraviolet Light. Nat. Phys. 2008, 4, 3-7.

(237) Yu, L.-H.; Babzien, M.; Ben-Zvi, I.; DiMauro, L. F.; Doyuran, A.; Graves, W.; Johnson, E.; Krinsky, S.; Malone, R.; Pogorelsky, I.; et al., High-Gain Harmonic-Generation Free-Electron Laser. Science 2000, 289, 932-934.

(238) Allaria, E.; Appio, R.; Badano, L.; Barletta, W.; Bassanese, S.; Biedron, S.; Borga, A.; Busetto, E.; Castronovo, D.; Cinquegrana, P.; et al., Highly Coherent and Stable Pulses from the FERMI Seeded Free-Electron Laser in the Extreme Ultraviolet. Nat. Photonics 2012, 6, 699-704.

(239) Chapman, H. N.; Barty, A.; Bogan, M. J.; Boutet, S.; Frank, M.; Hau-Riege, S. P.; Marchesini, S.; Woods, B. W.; Bajt, S.; Benner, W. H.; et al., Femtosecond Diffractive Imaging with a Soft-X-Ray Free-Electron Laser. Nat. Phys. 2006, 2, 839-843.

(240) Chapman, H. N.; Hau-Riege, S. P.; Bogan, M. J.; Bajt, S.; Barty, A.; Boutet, S.; Marchesini, S.; Frank, M.; Woods, B. W.; Benner, W. H.; et al., Femtosecond TimeDelay X-Ray Holography. Nature 2007, 448, 676-679.

(241) Moshammer, R.; Jiang, Y. H.; Foucar, L.; Rudenko, A.; Ergler, T.; Schröter, C. D.; Lüdemann, S.; Zrost, K.; Fischer, D.; Titze, J.; et al., Few-Photon Multiple Ionization of Ne and Ar by Strong Free-Electron-Laser Pulses. Phys. Rev. Lett. 2007, 98, 203001. 
(242) Erk, B.; Boll, R.; Trippel, S.; Anielski, D.; Foucar, L.; Rudek, B.; Epp, S. W.; Coffee, R.; Carron, S.; Schorb, S.; et al., Imaging Charge Transfer in Iodomethane upon X-Ray Photoabsorption. Science 2014, 345, 288-291.

(243) Jiang, Y. H.; Rudenko, A.; Herrwerth, O.; Foucar, L.; Kurka, M.; Kühnel, K. U.; Lezius, M.; Kling, M. F.; van Tilborg, J.; Belkacem, A.; et al., Ultrafast Extreme Ultraviolet Induced Isomerization of Acetylene Cations. Phys. Rev. Lett. 2010, 105, 263002.

(244) Magrakvelidze, M.; Herrwerth, O.; Jiang, Y. H.; Rudenko, A.; Kurka, M.; Foucar, L.; Kühnel, K. U.; Kübel, M.; Johnson, N. G.; Schröter, C. D.; et al., Tracing NuclearWave-Packet Dynamics in Singly and Doubly Charged States of $\mathrm{N}_{2}$ and $\mathrm{O}_{2}$ with XUV-Pump XUV-Probe Experiments. Phys. Rev. A 2012, 86, 013415.

(245) Bostedt, C.; Thomas, H.; Hoener, M.; Eremina, E.; Fennel, T.; Meiwes-Broer, K.-H.; Wabnitz, H.; Kuhlmann, M.; Plönjes, E.; Tiedtke, K.; et al., Multistep Ionization of Argon Clusters in Intense Femtosecond Extreme Ultraviolet Pulses. Phys. Rev. Lett. 2008, 100, 133401.

(246) Rohringer, N.; Ryan, D.; London, R. A.; Purvis, M.; Albert, F.; Dunn, J.; Bozek, J. D.; Bostedt, C.; Graf, A.; Hill, R.; et al., Atomic Inner-Shell X-Ray Laser at 1.46 nanometres Pumped by an X-Ray Free-Electron Laser. Nature 2012, 481, 488-491.

(247) Küpper, J.; Stern, S.; Holmegaard, L.; Filsinger, F.; Rouzée, A.; Rudenko, A.; Johnsson, P.; Martin, A. V.; Adolph, M.; Aquila, A.; et al., X-Ray Diffraction from Isolated and Strongly Aligned Gas-Phase Molecules with a Free-Electron Laser. Phys. Rev. Lett. 2014, 112, 083002.

(248) Bostedt, C.; Boutet, S.; Fritz, D. M.; Huang, Z.; Lee, H. J.; Lemke, H. T.; Robert, A.; Schlotter, W. F.; Turner, J. J.; Williams, G. J. Linac Coherent Light Source: The first five years. Rev. Mod. Phys. 2016, 88, 015007. 
(249) Feldhaus, J.; Krikunova, M.; Meyer, M.; Möller, T.; Moshammer, R.; Rudenko, A.; Tschentscher, T.; Ullrich, J. AMO science at the FLASH and European XFEL freeelectron laser facilities. J. Phys. B: At., Mol. Opt. Phys. 2013, 46, 164002.

(250) Helml, W.; Maier, A.; Schweinberger, W.; Grguraš, I.; Radcliffe, P.; Doumy, G.; Roedig, C.; Gagnon, J.; Messerschmidt, M.; Schorb, S.; et al., Measuring the Temporal Structure of Few-Femtosecond Free-Electron Laser X-Ray Pulses Directly in the Time Domain. Nat. Photonics 2014, 8, 950-957.

(251) Cederbaum, L. S.; Domcke, W.; Schirmer, J.; Niessen, W. V. Advances in Chem. Phys.; John Wiley \& Sons, Inc., 2007; pp 115-159.

(252) Vacher, M.; Bearpark, M.; Robb, M. Communication: Oscillating Charge Migration between Lone Pairs Persists without Significant Interaction with Nuclear Motion in the Glycine and Gly-Gly-NH-CH 3 Radical Cations. J. Chem. Phys. 2014, 140, 201102.

(253) Kuleff, A. I.; Cederbaum, L. S. Ultrafast Correlation-Driven Electron Dynamics. J. Phys. B: At., Mol. Opt. Phys.: At., Mol. Opt. Phys. 2014, 47, 124002.

(254) Calegari, F.; Ayuso, D.; Trabattoni, A.; Belshaw, L.; Camillis, S. D.; Frassetto, F.; Poletto, L.; Palacios, A.; Decleva, P.; Greenwood, J. B.; et al., Ultrafast Charge Dynamics in an Amino Acid Induced by Attosecond Pulses. IEEE J. Select. Top. Quantum Electron. 2015, 21, 8700512.

(255) Bachau, H.; Cormier, E.; Decleva, P.; Hansen, J. E.; Martín, F. Applications of Bsplines in atomic and Mol. Phys. Rep. Prog. Phys. 2001, 64, 1815.

(256) Stratmann, R. E.; Bandarage, G.; Lucchese, R. R. Electron-Correlation Effects in the Photoionization of $\mathrm{N}_{2}$. Phys. Rev. A 1995, 51, 3756-3765.

(257) Stratmann, R. E.; Zurales, R. W.; Lucchese, R. R. Multiplet-Specific Multichannel 
Electron-Correlation Effects in the Photoionization of NO. J. Chem. Phys. 1996, 104, 8989-9000.

(258) Rescigno, T. N.; Lengsfield, B. H.; Orel, A. E. Interchannel Coupling and Ground State Correlation Effects in the Photoionization of CO. J. Chem. Phys. 1993, 99, $5097-5103$.

(259) T. N. Rescigno, B. H. L. I.; McCurdy, C. W. Modern Electronic Structure Theory; Advances Series in Physical Chemistry; D R Yarkony (World Scientific, Singapore), 1995; Vol. 1; p 501.

(260) Haxton, D. J.; Lawler, K. V.; McCurdy, C. W. Multiconfiguration Time-Dependent Hartree-Fock Treatment of Electronic and Nuclear Dynamics in Diatomic Molecules. Phys. Rev. A 2011, 83, 063416.

(261) Haxton, D. J.; McCurdy, C. W. Two Methods for Restricted Configuration Spaces within the Multiconfiguration Time-Dependent Hartree-Fock Method. Phys. Rev. A 2015, 91, 012509 .

(262) Rescigno, T. N.; Trevisan, C. S.; McCurdy, C. W. Tracking Hole Localization in K-Shell and Core-Valence-Excited Acetylene Photoionization via Body-Frame Photoelectron Angular Distributions. Phys. Rev. A 2015, 91, 023429.

(263) Stener, M.; Decleva, P. Time-Dependent Density Functional Calculations of Molecular Photoionization Cross Sections: $\mathrm{N}_{2}$ and $\mathrm{Ph}_{3}$. J. Chem. Phys. 2000, 112, 10871-10879.

(264) D. Toffoli, G. F., M. Stener; Decleva, P. Convergence of the Multicenter B-Spline DFT Approach for the Continuum. Chem. Phys. 2002, 276, 25-43.

(265) Stener, M.; Fronzoni, G.; Decleva, P. Time-Dependent Density-Functional Theory for Molecular Photoionization with Noniterative Algorithm and Multicenter B-Spline Basis Set: $\mathrm{CS}_{2}$ and $\mathrm{C}_{6} \mathrm{H}_{6}$ Case Studies. J. Chem. Phys. 2005, 122, 234301. 
(266) Brambila, D. S.; Harvey, A. G.; Mašín, Z.; Gorfinkiel, J. D.; Smirnova, O. The Role of Multichannel Effects in the Photoionization of the $\mathrm{NO}_{2}$ molecule: an Ab Initio $\mathrm{R}$ -Matrix Study. J. Phys. B: At., Mol. Opt. Phys.: At., Mol. Opt. Phys. 2015, 48, 245101.

(267) Kuleff, A. I.; Breidbach, J.; Cederbaum, L. S. Multielectron Wave-Packet Propagation: General Theory and Application. J. Chem. Phys. 2005, 123, 0441111.

(268) Vacher, M.; Steinberg, L.; Jenkins, A. J.; Bearpark, M. J.; Robb, M. A. Electron Dynamics Following Photoionization: Decoherence due to the Nuclear-Wave-Packet Width. Phys. Rev. A 2015, 92, 040502.

(269) Kryachko, E. S.; Ludeña, E. V. Energy Density Functional Theory of Many-Electron Systems; Springer, 1990; pp 82-90.

(270) Nesbet, R. K. Variational Methods in Electron-Atom Scattering Theory; Plenum, New York, Springer, 1980; pp 82-90.

(271) Gianturco, F. A.; Huo, W. Computational Methods for Electron-Molecule Collisions; Plenum, NewYork, Springer, 1995.

(272) Burke, P. G. R-Matrix Theory of Atomic Collisions; Springer-Verlag Berlin Heidelberg, 2011.

(273) Breit, G.; Bethe, H. A. Ingoing Waves in Final State of Scattering Problems. Phys. Rev. 1954, 93, 888-890.

(274) Starace, A. F. In Handbuch der Physik; Mehlhorn, W., Ed.; Springer-Verlag, Berlin, 1982; Vol. 31; pp 1-121.

(275) Pickup, B. T. On the Theory of Fast Photoionization Processes. Chem. Phys. 1977, 19, $193-208$. 
(276) McWeeny, R. Methods of Molecular Quantum Mechanics; Theoretical chemistry; Academic Press, 1992.

(277) Martin, R. L.; Shirley, D. A. Theory of the Neon 1s Correlation-Peak Intensities. Phys. Rev. A 1976, 13, 1475-1483.

(278) Arneberg, R.; Mueller, J.; Manne, R. Configuration Interaction Calculations of Satellite Structure in Photoelectron Spectra of $\mathrm{H}_{2} \mathrm{O}$. Chem. Phys. 1982, 64, $249-258$.

(279) Ponzi, A.; Angeli, C.; Cimiraglia, R.; Coriani, S.; Decleva, P. Dynamical Photoionization Observables of the CS Molecule: the Role of Electron Correlation. J. Chem. Phys. 2014, 140, 204304.

(280) Oana, C. M.; Krylov, A. I. Cross Sections and Photoelectron Angular Distributions in Photodetachment from Negative Ions Using Equation-Of-Motion Coupled-Cluster Dyson Orbitals. J. Chem. Phys. 2009, 131, 124114.

(281) Miao, Y. R.; Ning, C. G.; Liu, K.; Deng, J. K. Dyson Orbitals of $\mathrm{N}_{2} \mathrm{O}$ : Electron Momentum Spectroscopy and Symmetry Adapted Cluster-Configuration Interaction Calculations. J. Chem. Phys. 2011, 134, 204304.

(282) Spanner, M.; Patchkovskii, S. Molecular Strong Field Ionization and High Harmonic Generation: A Selection of Computational Illustrations. Chem. Phys. 2013, 414, 1019.

(283) Humeniuk, A.; Wohlgemuth, M.; Suzuki, T.; Mitrić, R. Time-Resolved Photoelectron Imaging Spectra from Non-Adiabatic Molecular Dynamics Simulations. J. Chem. Phys. 2013, 139, 134104.

(284) Padial, N.; Csanak, G.; McKoy, B. V.; Langhoff, P. W. Photoexcitation and Ionization in Carbon Dioxide: Theoretical Studies in the Separated-Channel Static-Exchange Approximation. Phys. Rev. A 1981, 23, 218-235. 
(285) McKoy, V.; Carlson, T. A.; Lucchese, R. R. Photoelectron Dynamics of Molecules. J. Phys. Chem. 1984, 88, 3188-3196.

(286) Kohn, W.; Sham, L. J. Self-Consistent Equations Including Exchange and Correlation Effects. Phys. Rev. 1965, 140, A1133-A1138.

(287) Plésiat, E.; Decleva, P.; Martin, F. Vibrational branching ratios in the photoelectron spectra of $\mathrm{N}_{2}$ and CO: interference and diffraction effects. Phys. Chem. Chem. Phys. 2012, 14, 10853-10871.

(288) Ellison, F. O. Theoretical Equations for Photoionization Cross Sections of Polyatomic Molecules in Plane-Wave and Orthogonalized Plane-Wave Approximations. J. Chem. Phys. 1974, 61, 507-515.

(289) Rabalais, J. W.; Debies, T. P.; Berkosky, J. L.; Huang, J. J.; Ellison, F. O. Calculated Photoionization Cross Sections and Relative Experimental Photoionization Intensities for a Selection of Small Molecules. J. Chem. Phys. 1974, 61, 516-528.

(290) Rabalais, J. W.; Debies, T. P.; Berkosky, J. L.; Huang, J. J.; Ellison, F. O. Erratum: Calculated Angular Distributions of Photoelectrons Using the Orthogonalized PlaneWave Approximation. J. Chem. Phys. 1975, 62, 4588-4588.

(291) Williams, R. S.; Shirley, D. A. Comparison of Final State Approximations in the Calculation of Total and Differential Photoemission Cross Sections of Neon. J. Chem. Phys. 1977, 66, 2378-2386.

(292) Gozem, S.; Gunina, A. O.; Ichino, T.; Osborn, D. L.; Stanton, J. F.; Krylov, A. I. Photoelectron Wave Function in Photoionization: Plane Wave or Coulomb Wave? J. Phys. Chem. Letters 2015, 6, 4532-4540.

(293) Mignolet, B.; Levine, R. D.; Remacle, F. Localized Electron Dynamics in Attosecond- 
Pulse-Excited Molecular Systems: Probing the Time-Dependent Electron Density by Sudden Photoionization. Phys. Rev. A 2012, 86, 053429.

(294) Mignolet, B.; Levine, R. D.; Remacle, F. Charge Migration in the Bifunctional PENNA Cation Induced and Probed by Ultrafast Ionization: A Dynamical Study. J. Phys. B: At., Mol. Opt. Phys. 2014, 47, 124011.

(295) Mignolet, B.; Levine, R. D.; Remacle, F. Control of Electronic Dynamics Visualized by Angularly Resolved Photoelectron Spectra: A Dynamical Simulation with an IR Pump and XUV Attosecond-Pulse-Train Probe. Phys. Rev. A 2014, 89, 021403.

(296) Ponzi, A.; Sapunar, M.; Angeli, C.; Cimiraglia, R.; Došlić, N.; Decleva, P. Photoionization of Furan from the Ground and Excited Electronic States. J. Chem. Phys. 2016, $144,084307$.

(297) Demekhin, P. V.; Ehresmann, A.; Sukhorukov, V. L. Single Center Method: A Computational Tool for Ionization and Electronic Excitation Studies of Molecules. J. Chem. Phys. 2011, 134, 024113.

(298) Schneider, B. I.; Rescigno, T. N. Complex Kohn Variational Method: Application to Low-Energy Electron-Molecule Collisions. Phys. Rev. A 1988, 37, 3749-3754.

(299) Lucchese, R. R.; Raseev, G.; McKoy, V. Studies of Differential and Total Photoionization Cross Sections of Molecular Nitrogen. Phys. Rev. A 1982, 25, 2572-2587.

(300) Lucchese, R. R.; Takatsuka, K.; McKoy, V. Applications of the Schwinger Variational Principle to Electron-Molecule Collisions and Molecular Photoionization. Phys. Rep. 1986, $131,147-221$.

(301) Martín, F. Ionization and Dissociation Using B-Splines: Photoionization of the Hydrogen Molecule. J. Phys. B: At., Mol. Opt. Phys. 1999, 32, R197-R231. 
(302) Froese Fischer, C.; Idrees, M. Spline Algorithms for Continuum Functions. Comput. Phys. 1989, 3, 53-58.

(303) Brosolo, M.; Decleva, P. Variational Approach to Continuum Orbitals in a Spline Basis: an Application to $\mathrm{H}_{2}^{+}$photoionization. Chem. Phys. 1992, 159, 185 - 196.

(304) Brosolo, M.; Decleva, P.; Lisini, A. Accurate variational determination of continuum wavefunctions by a one-centre expansion in a spline basis. An application to $\mathrm{H}_{2}^{+}$and $\mathrm{HeH}_{2}^{+}$photoionization. J. Phys. B: At., Mol. Opt. Phys.: At., Mol. Opt. Phys. 1992, $25,3345-3356$.

(305) Marante, C.; Klinker, M.; Corral, I.; Argenti, L.; González-Vázquez, J.; Martín, F. to be published 2016,

(306) Dill, D.; Dehmer, J. L. Electron-Molecule Scattering and Molecular Photoionization Using the Multiple-Scattering Method. J. Chem. Phys. 1974, 61, 692-699.

(307) Suzuki, Y.; Suzuki, T. Photoelectron Angular Distribution in Valence Shell Ionization of Heteroaromatic Molecules Studied by the Continuum Multiple Scattering X $\alpha$ Method. J. Phys. Chem. A 2008, 112, 402-411.

(308) Powis, I. Initial-State And Final-State Vibrational Effects in the Treatment of Molecular Photoionization Dynamics. Phys. Rev. A 2011, 84, 013402.

(309) Son, S.-K.; Chu, S.-I. Theoretical Study of Orientation-Dependent Multiphoton Ionization of Polyatomic Molecules in Intense Ultrashort Laser Fields: A New TimeDependent Voronoi-Cell Finite Difference Method. Chem. Phys. 2009, 366, 91 - 102.

(310) Rohringer, N.; Gordon, A.; Santra, R. Configuration-Interaction-Based TimeDependent Orbital Approach for ab initio Treatment of Electronic Dynamics in a Strong Optical Laser Field. Phys. Rev. A 2006, 74, 043420. 
(311) Greenman, L.; Ho, P. J.; Pabst, S.; Kamarchik, E.; Mazziotti, D. A.; Santra, R. Implementation of the Time-Dependent Configuration-Interaction Singles Method for Atomic Strong-Field Processes. Phys. Rev. A 2010, 82, 023406.

(312) Pabst, S.; Greenman, L.; Ho, P. J.; Mazziotti, D. A.; Santra, R. Decoherence in Attosecond Photoionization. Phys. Rev. Lett. 2011, 106, 053003.

(313) Karamatskou, A.; Pabst, S.; Chen, Y.-J.; Santra, R. Calculation of Photoelectron Spectra within the Time-Dependent Configuration-Interaction Singles Scheme. Phys. Rev. A 2014, 89, 033415 .

(314) Schlegel, H. B.; Smith, S. M.; Li, X. Electronic Optical Response of Molecules in Intense Fields: Comparison of TD-HF, TD-CIS, and TD-CIS(D) Approaches. J. Chem. Phys. 2007, 126, 244110.

(315) Sonk, J. A.; Schlegel, H. B. TD-CI Simulation of the Electronic Optical Response of Molecules in Intense Fields II: Comparison of DFT Functionals and EOM-CCSD. $J$. Phys. Chem. A 2011, 115, 11832-11840.

(316) Krause, P.; Schlegel, H. B. Angle-Dependent Ionization of Hydrides AHn Calculated by Time-Dependent Configuration Interaction with an Absorbing Potential. J. Phys. Chem. A 2015, 119, 10212-10220.

(317) Krause, P.; Schlegel, H. B. Angle-Dependent Ionization of Small Molecules by TimeDependent Configuration Interaction and an Absorbing Potential. J. Phys. Chem. Letters 2015, 6, 2140-2146.

(318) Toffoli, D.; Decleva, P. to be published 2016,

(319) Cacelli, I.; Carravetta, V.; Moccia, R.; Rizzo, A. Photoionization and Photoabsorption Cross Section Calculations in Methane, Ammonia, Water, and Hydrogen Fluoride Molecules. J. Phys. Chem. 1988, 92, 979-982. 
(320) Semenov, S. K.; Cherepkov, N. A.; Fecher, G. H.; Schönhense, G. Generalization of the Atomic Random-Phase-Approximation Method for Diatomic Molecules: $\mathrm{N}_{2}$ Photoionization Cross-Section Calculations. Phys. Rev. A 2000, 61, 032704.

(321) Stener, M.; Toffoli, D.; Fronzoni, G.; Decleva, P. Time Dependent Density Functional Study of the Photoionization Dynamics of $\mathrm{SF}_{6}$. J. Chem. Phys. 2006, 124 .

(322) Catone, D.; Stener, M.; Decleva, P.; Contini, G.; Zema, N.; Prosperi, T.; Feyer, V.; Prince, K. C.; Turchini, S. Resonant Circular Dichroism of Chiral Metal-Organic Complex. Phys. Rev. Lett. 2012, 108, 083001.

(323) Tennyson, J. Electron-Molecule Collision Calculations Using the -Matrix Method. Phys. Rep. 2010, 491, $29-76$.

(324) Yip, F. L.; McCurdy, C. W.; Rescigno, T. N. Hybrid Gaussian Discrete-Variable Representation for One- and Two-Active-Electron Continuum Calculations in Molecules. Phys. Rev. A 2014, 90, 063421.

(325) Lin, C.-Y.; McCurdy, C. W.; Rescigno, T. N. Complex Kohn Approach To Molecular Ionization By High-Energy Electrons: Application to $\mathrm{H}_{2}$ O. Phys. Rev. A 2014, 89, 012703.

(326) Majety, V. P.; Zielinski, A.; Scrinzi, A. Photoionization of few electron systems: a hybrid coupled channels approach. New J. Phys. 2015, 17, 063002.

(327) Chattopadhyay, S.; Bauch, S.; Madsen, L. B. Electron-Correlation Effects in Enhanced Ionization of Molecules: A Time-Dependent Generalized-Active-Space ConfigurationInteraction Study. Phys. Rev. A 2015, 92, 063423.

(328) Marante, C.; Kjellsson, T.; Klinker, M.; Corral, I.; Lindroth, E.; González-Vázquez, J.; Argenti, L.; Martín, F. to be published 2016, 
(329) Marante, C.; Argenti, L.; Martín, F. Hybrid Gaussian-B-Spline Basis for the Electronic Continuum: Photoionization of Atomic Hydrogen. Phys. Rev. A 2014, 90, 012506.

(330) Jose, J.; Lucchese, R. R.; Rescigno, T. N. Interchannel Coupling Effects in the Valence Photoionization of $\mathrm{SF}_{6}$. J. Chem. Phys. 2014, 140, 204305.

(331) Majety, V. P.; Scrinzi, A. Dynamic Exchange in the Strong Field Ionization of Molecules. Phys. Rev. Lett. 2015, 115, 103002.

(332) de Boor, C. A Practical Guide to Splines, Applied Mathematical Sciences 27.; SpringerVerlag, New York, 1978.

(333) Brosolo, M.; Decleva, P.; Lisini, A. Continuum Wavefunctions Calculations with LeastSquares Schemes in a B-Splines Basis. Comput. Phys. Commun. 1992, 71, $207-214$.

(334) Casida, M. E. In Recent Developments and Applications of Modern Density Functional Theory, 1st Edition; Seminario, J. M., Ed.; Elsevier Elsevier Science, Amsterdam, $1996 ;$ p 391.

(335) Stener, M.; Toffoli, D.; Fronzoni, G.; Decleva, P. Recent Advances in Molecular Photoionization by Density Functional Theory Based Approaches. Theor. Chem. Acc. 2007, 117, 943-956.

(336) Canton, S. E.; Plesiat, E.; Bozek, J. D.; Rude, B. S.; Decleva, P.; Martin, F. Direct Observation of Young's Double-Slit Interferences in Vibrationally Resolved Photoionization of Diatomic Molecules. Proceedings of the National Academy of Sciences 2011, $108,7302-7306$.

(337) Kushawaha, R. K.; Patanen, M.; Guillemin, R.; Journel, L.; Miron, C.; Simon, M.; Piancastelli, M. N.; Skates, C.; Decleva, P. From Double-Slit Interference to Structural Information in Simple Hydrocarbons. Proceedings of the National Academy of Sciences 2013, 110, 15201-15206. 
(338) Kuleff, A. I.; Lünnemann, S.; Cederbaum, L. S. Electron-Correlation-Driven Charge Migration in Oligopeptides. Chem. Phys. 2013, 414, $100-105$.

(339) Lünnemann, S.; Kuleff, A. I.; Cederbaum, L. S. Ultrafast Electron Dynamics Following Outer-Valence Ionization: The Impact of Low-Lying Relaxation Satellite States. J. Chem. Phys. 2009, 130, 154305.

(340) Cederbaum, L. S.; Domcke, W. Theoretical aspects of ionization potentials and photoelectron spectroscopy: a many-body approach. Advances in Chem. Phys. 1977, 36, 205.

(341) Öhrn, Y.; Born, G. Molecular Electron Propagator Theory and Calculations; Advances in Quantum Chemistry; Academic Press, 1981; Vol. 13; pp 1 - 88.

(342) Schirmer, J.; Cederbaum, L. S.; Walter, O. New Approach to the One-Particle Green's Function for Finite Fermi Systems. Phys. Rev. A 1983, 28, 1237-1259.

(343) Schirmer, J. Closed-Form Intermediate Representations of Many-Body Propagators and Resolvent Matrices. Phys. Rev. A 1991, 43, 4647-4659.

(344) Schirmer, J.; Trofimov, A. B.; Stelter, G. A Non-Dyson Third-Order Approximation Scheme for the Electron Propagator. J. Chem. Phys. 1998, 109, 4734-4744.

(345) Musia, M.; Bartlett, R. J. EOM-CCSDT Study of the Low-Lying Ionization Potentials of Ethylene, Acetylene and Formaldehyde. Chem. Phys. Lett. 2004, 384, 210 - 214.

(346) Kamiya, M.; Hirata, S. Higher-Order Equation-of-Motion Coupled-Cluster Methods for Ionization Processes. J. Chem. Phys. 2006, 125, 074111.

(347) Nakatsuji, H. Description of Two- and Many-Electron Processes by the SAC-CI Method. Chem. Phys. Lett. 1991, 177, $331-337$. 
(348) Ehara, M.; Nakatsuji, H. Outer- and Inner-Valence Ionization Spectra Of $\mathrm{N}_{2}$ and CO:: SAC-CI (General-R) Compared with Full-CI Spectra. Chem. Phys. Lett. 1998, 282, $347-354$.

(349) Corral, I.; González-Vázquez, J.; Martín, F. to be published 2016,

(350) Lischka, H. et al. COLUMBUS, an ab initio Electronic Structure Program, Release 7.0. (accessed 2015); http://www.univie.ac.at/columbus/.

(351) Werner, H.-J. et al. MOLPRO, version 2015.1, a package of ab initio programs. http://www.molpro.net/, 2015.

(352) Aquilante, F. et al. MOLCAS 8. http://www.molcas.org/, 2016; Journal of Computational Chemistry, 37, 506-541.

(353) Bawagan, A. D. O.; Davidson, E. R. Advances in Chem. Phys.; John Wiley and Sons, Inc., 2007; pp 215-266.

(354) Decleva, P.; Ohno, M. Many-Body Calculation of the XPS Core Hole Spectra of $\mathrm{NiN}_{2}$. Chem. Phys. 1992, 160, $341-351$.

(355) Ohno, M.; Decleva, P. New Interpretation of the Core Hole Spectra of CO and $\mathrm{N}_{2}$ Adsorbed on a Ni Metal Surface. Surf. Sci. 1992, 269-270, $264-269$.

(356) Breidbach, J.; Cederbaum, L. S. Migration of Holes: Formalism, Mechanisms, and Illustrative Applications. J. Chem. Phys. 2003, 118, 3983-3996.

(357) Kuleff, A. I.; Cederbaum, L. S. Charge Migration in Different Conformers of Glycine: The Role of Nuclear Geometry. Chem. Phys. 2007, 338, 320-328.

(358) Lünnemann, S.; Kuleff, A. I.; Cederbaum, L. S. Ultrafast Charge Migration in 2Phenylethyl-N,N-Dimethylamine. Chem. Phys. Lett. 2008, 450, 232-235. 
(359) Lara-Astiaso, M.; Ayuso, D.; Tavernelli, I.; Decleva, P.; Palacios, A.; F., M. Decoherence, Control and Attosecond Probing of XUV-Induced Charge Migration in Biomolecules. A Theoretical Outlook. Faraday Discuss. 2016, (in press).

(360) Ayuso, D.; Palacios, A.; Decleva, P.; Martín, F. to be published 2016,

(361) Ayuso, D.; Kimura, M.; Kooser, K.; Patanen, M.; Plésiat, E.; Argenti, L.; Mondal, S.; Travnikova, O.; Sakai, K.; Palacios, A.; et al., Vibrationally Resolved B 1s Photoionization Cross Section of BF 3. J. Phys. Chem. A 2015, 119, 5971-5978.

(362) Plésiat, E.; Argenti, L.; Kukk, E.; Miron, C.; Ueda, K.; Decleva, P.; Martín, F. Intramolecular Electron Diffraction in Vibrationally Resolved K-Shell Photoionization of Methane. Phys. Rev. A 2012, 85, 023409.

(363) Ueda, K.; Miron, C.; Plésiat, E.; Argenti, L.; Patanen, M.; Kooser, K.; Ayuso, D.; Mondal, S.; Kimura, M.; Sakai, K.; et al., Intramolecular Photoelectron Diffraction in the Gas Phase. J. Chem. Phys. 2013, 139, 124306.

(364) Plésiat, E.; Decleva, P.; Martín, F. Relationship Between Polarization-Averaged Molecular-Frame Photoelectron Angular Distributions and Geometry. Phys. Rev. A 2013, 88, 063409 .

(365) Barbatti, M.; Aquino, A. J. a.; Szymczak, J. J.; Nachtigallova, D.; Hobza, P.; Lischka, H. Relaxation Mechanisms of UV-Photoexcited DNA and RNA Nucleobases. Proceedings of the National Academy of Sciences 2010, 10\%, 21453-21458.

(366) Richter, M.; Marquetand, P.; González-Vázquez, J.; Sola, I.; González, L. Femtosecond Intersystem Crossing in the DNA Nucleobase Cytosine. J. Phys. Chem. Letters 2012, 3, 3090-3095.

(367) Persico, M.; Granucci, G. An Overview of Nonadiabatic Dynamics Simulations Meth- 
ods, with Focus on the Direct Approach Versus the Fitting of Potential Energy Surfaces. Theor. Chem. Acc. 2014, 133, 1526.

(368) Tavernelli, I.; Röhrig, U. F.; Rothlisberger, U. Molecular Dynamics in Electronically Excited States Using Time-Dependent Density Functional Theory. Mol. Phys. 2005, 103, 963-981.

(369) Tavernelli, I. Electronic Density Response of Liquid Water Using Time-Dependent Density Functional Theory. Phys. Rev. B 2006, 73, 094204.

(370) CPMD, Copyright IBM Corp 1990-2015, Copyright MPI fuer Festkoerperforschung Stuttgart 1997-2001. http://www.cpmd.org/, 2014.

(371) Hammes-Schiffer, S.; Tully, J. C. Proton Transfer in Solution: Molecular Dynamics with Quantum Transitions. J. Chem. Phys. 1994, 101, 4657-4667.

(372) Hammes-Schiffer, S.; Tully, J. C. Nonadiabatic Transition State Theory and Multiple Potential Energy Surface Molecular Dynamics of Infrequent Events. J. Chem. Phys. 1995, 103, 8528-8537.

(373) Richter, M.; Marquetand, P.; González-Vázquez, J.; Sola, I.; González, L. SHARC: ab Initio Molecular Dynamics with Surface Hopping in the Adiabatic Representation Including Arbitrary Couplings. J. Chem. Theory Comput. 2011, 7, 1253-1258.

(374) Tully, J. C. Perspective: Nonadiabatic dynamics theory. J. Chem. Phys. 2012, 137, $22 \mathrm{~A} 301$.

(375) Curchod, B. F. E.; Rothlisberger, U.; Tavernelli, I. Trajectory-Based Nonadiabatic Dynamics with Time-Dependent Density Functional Theory. Chem. Phys. Chem. 2013, 14, 1314-1340.

(376) Despré, V.; Marciniak, A.; Loriot, V.; Galbraith, M. C. E.; Rouzée, A.; Vrakking, M. 
J. J.; Lépine, F.; Kuleff, A. I. Attosecond Hole Migration in Benzene Molecules Surviving Nuclear Motion. Journal of Physical Chemistry Letters 2015, 6, 426-431.

(377) Vacher, M.; Mendive-Tapia, D.; Bearpark, M. J.; Robb, M. A. The Second-Order Ehrenfest Method. Theor. Chem. Acc. 2014, 133, 1505.

(378) Bian, X.-B.; Bandrauk, A. D. Attosecond Time-Resolved Imaging of Molecular Structure by Photoelectron Holography. Phys. Rev. Lett. 2012, 108, 263003.

(379) Bian, X.-B.; Bandrauk, A. D. Probing nuclear motion by frequency modulation of molecular high-order harmonic generation. Phys. Rev. Lett. 2014, 113, 193901.

(380) Lara-Astiaso, M.; Silva, R. E. F.; Gubaydullin, A.; Rivière, P.; Meier, C.; Martín, F. to be published 2016,

(381) Lein, M. Attosecond probing of vibrational dynamics with high-harmonic generation. Phys. Rev. Lett. 2005, 94, 053004.

(382) Posthumus, J. H. The Dynamics of Small Molecules in Intense Laser Fields. Rep. Prog. Phys. 2004, 67, 623-665.

(383) Calvert, C.; Bryan, W.; Newell, W.; Williams, I. Time-Resolved Studies of Ultrafast Wavepacket Dynamics in Hydrogen Molecules. Phys. Rep. 2010, 491, 1-28.

(384) Bandrauk, A. D.; Chelkowski, S.; Nguyen, H. S. Attosecond Localization of Electrons in Molecules. Int. J. Quantum Chem. 2004, 100, 834-844.

(385) Kling, M. F.; Siedschlag, C.; Verhoef, a. J.; Khan, J. I.; Schultze, M.; Uphues, T.; Ni, Y.; Uiberacker, M.; Drescher, M.; Krausz, F.; Vrakking, M. J. J. Control of Electron Localization in Molecular Dissociation. Science 2006, 312, 246-248.

(386) Singh, K. P.; He, F.; Ranitovic, P.; Cao, W.; De, S.; Ray, D.; Chen, S.; Thumm, U.; Becker, A.; Murnane, M. M.; et al., Control of Electron Localization in Deuterium 
Molecular Ions using an Attosecond Pulse Train and a Many-Cycle Infrared Pulse. Phys. Rev. Lett. 2010, 104, 023001.

(387) Sánchez, I.; Martín, F. Multichannel Dissociation in Resonant Photoionization of $\mathrm{H}_{2}$. Phys. Rev. Lett. 1999, 82, 3775.

(388) Palacios, A.; Feist, J.; González-Castrillo, A.; Sanz-Vicario, J. L.; Martín, F. Autoionization of Molecular Hydrogen: where do the Fano Lineshapes Go? Chem. Phys. Chem. 2013, 14, 1456-63.

(389) Kelkensberg, F.; Siu, W.; Pérez-Torres, J.; Morales, F.; Gademann, G.; Rouzée, A.; Johnsson, P.; Lucchini, M.; Calegari, F.; Sanz-Vicario, J.; et al., Attosecond Control in Photoionization of Hydrogen Molecules. Phys. Rev. Lett. 2011, 107, 043002.

(390) Ranitovic, P.; Hogle, C. W.; Riviere, P.; Palacios, A.; Tong, X.-M.; Toshima, N.; Gonzalez-Castrillo, A.; Martin, L.; Martin, F.; Murnane, M. M.; Kapteyn, H. Attosecond Vacuum UV Coherent Control of Molecular Dynamics. Proceedings of the National Academy of Sciences 2014, 111, 912-917.

(391) Bostedt, C.; Chapman, H. N.; Costello, J. T.; Crespo López-Urrutia, J. R.; Düsterer, S.; Epp, S. W.; Feldhaus, J.; Föhlisch, A.; Meyer, M.; Möller, T. Experiments at FLASH. Nucl. Instrum. Methods Phys. Res., Sect. A 2009, 601, 108-122.

(392) Chapman, H.; Ullrich, J.; Rost, J. M. Intense X-ray Science: the First 5 Years of FLASH. J. Phys. B: At., Mol. Opt. Phys.: At., Mol. Opt. Phys. 2010, 43, 190201.

(393) Jiang, Y. H. et al. Investigating Two-Photon Double Ionization of $\mathrm{D}_{2}$ by XUV-PumpXUV-Probe Experiments. Phys. Rev. A 2010, 81, 051402.

(394) Jiang, Y. H. et al. Tracing Direct and Sequential Two-Photon Double Ionization of $\mathrm{D}_{2}$ in Femtosecond Extreme-Ultraviolet Laser Pulses. Phys. Rev. A 2010, 81, 021401. 
(395) Jiang, Y. H. et al. Ultrafast dynamics in acetylene clocked in a femtosecond XUV stopwatch. J. Phys. B: At., Mol. Opt. Phys.: At., Mol. Opt. Phys. 2013, 46, 164027.

(396) Grguras, I.; Maier, A. R.; Behrens, C.; Mazza, T.; Kelly, T. J.; Radcliffe, P.; Dusterer, S.; Kazansky, A. K.; Kabachnik, N. M.; Tschentscher, T.; et al., Ultrafast X-Ray Pulse Characterization at Free-Electron Lasers. Nat. Photonics 2012, 6, 852-857.

(397) De Ninno, G.; Gauthier, D.; Mahieu, B.; Ribič, P. R.; Allaria, E.; Cinquegrana, P.; Danailov, M. B.; Demidovich, A.; Ferrari, E.; Giannessi, L.; et al., Single-Shot SpectroTemporal Characterization of XUV Pulses from a Seeded Free-Electron Laser. Nat. Commun. 2015, 6, 8075 .

(398) Gauthier, D.; Ribič, P. R.; De Ninno, G.; Allaria, E.; Cinquegrana, P.; Danailov, M. B.; Demidovich, A.; Ferrari, E.; Giannessi, L.; Mahieu, B.; et al., Spectrotemporal Shaping of Seeded Free-Electron Laser Pulses. Phys. Rev. Lett. 2015, 115, 114801.

(399) C., P. K. et al. Coherent Control with a Short-Wavelength Free-Electron Laser. Nat. Photonics 2016, 10, 176-179.

(400) Takahashi, E. J.; Lan, P.; Mücke, O. D.; Nabekawa, Y.; Midorikawa, K. Attosecond Nonlinear Optics Using Gigawatt-Scale Isolated Attosecond Pulses. Nat. Commun. 2013, 4, 2691.

(401) González-Castrillo, A.; Palacios, A.; Bachau, H.; Martín, F. Clocking Ultrafast Wave Packet Dynamics in Molecules through UV-Induced Symmetry Breaking. Phys. Rev. Lett. 2012, 108, 063009.

(402) Palacios, A.; González-Castrillo, A.; Martín, F. Molecular Interferometer to Decode Attosecond Electron-Nuclear Dynamics. Proc. Natl. Acad. Sci. U.S.A. 2014, 111, 3973-3978. 
(403) Feist, J.; Nagele, S.; Ticknor, C.; Schneider, B. I.; Collins, L. a.; Burgdörfer, J. Attosecond Two-Photon Interferometry for Doubly Excited States of Helium. Phys. Rev. Lett. 2011, 107, 093005.

(404) Fabris, D.; Witting, T.; Okell, W.; Walke, D.; Matia-Hernando, P.; Henkel, J.; Barillot, T.; Lein, M.; Marangos, J.; Tisch, J. Synchronized Pulses Generated at $20 \mathrm{eV}$ and $90 \mathrm{eV}$ for Attosecond Pump-Probe Experiments. Nat. Photonics 2015, 9, 383-387.

(405) Siu, W.; Kelkensberg, F.; Gademann, G.; Rouzée, A.; Johnsson, P.; Dowek, D.; Lucchini, M.; Calegari, F.; Giovannini, U. D.; Rubio, A.; et al., Attosecond Control of Dissociative Ionization of $\mathrm{O}_{2}$ Molecules. Phys. Rev. A 2011, 84, 063412.

(406) Cörlin, P.; Fischer, A.; Schonwald, M.; Sperl, A.; Mizuno, T.; Thumm, U.; Pfeifer, T.; Moshammer, R. Probing Calculated $\mathrm{O}_{2}^{+}$Potential-Energy Curves with an XUV-IR Pump-Probe Experiment. Phys. Rev. A 2015, 91, 043415.

(407) Sandhu, A. S.; Gagnon, E.; Santra, R.; Sharma, V.; Li, M.; Ho, P.; Ranitovic, P.; Cocke, C. L.; Murnane, M.; Kapteyn, H. C. Observing the Creation of Electronic Feshbach Resonances in Soft X-Ray-Induced $\mathrm{O}_{2}$ Dissociation. Science 2008, 322, 10811085 .

(408) Znakovskaya, I.; von den Ho, P.; Zherebtsov, S.; Wirth, A.; Herrwerth, O.; Vrakking, M. J. J.; de Vivie-Riedle, R.; Kling, M. F. Attosecond Control of Electron Dynamics in Carbon Monoxide. Phys. Rev. Lett. 2009, 103, 103002.

(409) Lucchini, M.; Kim, K.; Calegari, F.; Kelkensberg, F.; Siu, W.; Sansone, G.; Vrakking, M. J. J.; Hochlaf, M.; Nisoli, M. Autoionization and Ultrafast Relaxation Dynamics of Highly Excited States in $\mathrm{N}_{2}$. Phys. Rev. A 2012, 86, 043404.

(410) Eckstein, M.; Yang, C. H.; Kubin, M.; Frassetto, F.; Poletto, L.; Ritze, H. H.; Vrakking, M. J. J.; Kornilov, O. Dynamics of $\mathrm{N}_{2}$ Dissociation upon Inner-Valence 
Ionization by Wavelength-Selected XUV Pulses. J. Phys. Chem. Lett. 2015, 6, 419425.

(411) Gray, H. B.; Winkler, J. R. Electron Tunneling through Proteins. Q. Rev. Biophys. 2003, 36, 341-372.

(412) Willner, I.; Katz, E. Bioelectronics; Wiley-VCH Verlag GmbH \& Co. KGaA, 2005; pp $1-13$.

(413) Winkler, J. R.; Gray, H. B.; Prytkova, T. R.; Kurnikov, I. V.; Beratan, D. N. Bioelectronics; Wiley-VCH Verlag GmbH \& Co. KGaA, 2005; pp 15-33.

(414) Barbara, P. F.; Meyer, T. J.; Ratner, M. a. Contemporary Issues in Electron Transfer Research. J. Phys. Chem. 1996, 100, 13148-13168.

(415) Ikeura-Sekiguchi, H.; Sekiguchi, T. Attosecond Electron Delocalization in the Conduction Band through the Phosphate Backbone of Genomic DNA. Phys. Rev. Lett. 2007, 99, 228102.

(416) Calegari, F.; Trabattoni, A.; Palacios, A.; Ayuso, D.; Castrovilli, M. C.; Greenwood, J. B.; Decleva, P.; Martín, F.; Nisoli, M. Charge migration induced by attosecond pulses in bio-relevant molecules. J. Phys. B: At., Mol. Opt. Phys.: At., Mol. Opt. Phys. 2016, 49, 142001.

(417) Weinkauf, R.; Schanen, P.; Yang, D.; Soukara, S.; Schlag, E. W. Elementary Processes in Peptides: Electron Mobility and Dissociation in Peptide Cations in the Gas Phase. J. Phys. Chem. 1995, 99, 11255-11265.

(418) Weinkauf, R.; Schanen, P.; Metsala, A.; Schlag, E. W.; Bürgle, M.; Kessler, H. Highly Efficient Charge Transfer in Peptide Cations in the Gas Phase: Threshold Effects and Mechanism. J. Phys. Chem. 1996, 100, 18567-18585. 
(419) Weinkauf, R.; Schlag, E. W.; Martinez, T. J.; Levine, R. D. Nonstationary Electronic States and Site-Selective Reactivity. J. Phys. Chem. A 1997, 101, 7702-7710.

(420) Lehr, L.; Horneff, T.; Weinkauf, R.; Schlag, E. W. Femtosecond Dynamics After Ionization: 2-Phenylethyl-N,N-Dimethylamine as a Model System for Nonresonant Downhill Charge Transfer in Peptides. J. Phys. Chem. A 2005, 109, 8074-8080.

(421) Cheng, W.; Kuthirummal, N.; Gosselin, J. L.; Sølling, T. I.; Weinkauf, R.; Weber, P. M. Control of Local Ionization and Charge Transfer in the Bifunctional Molecule 2-Phenylethyl-N,N-Dimethylamine Using Rydberg Fingerprint Spectroscopy. J. Phys. Chem. A 2005, 109, 1920-1925.

(422) Marciniak, A.; Despré, V.; Barillot, T.; Rouzée, A.; Galbraith, M.; Klei, J.; Yang, C.H.; Smeenk, C.; Loriot, V.; Reddy, S. N.; et al., XUV Excitation Followed by Ultrafast Non-Adiabatic Relaxation in PAH Molecules as a Femto-Astrochemistry Experiment. Nat. Commun. 2015, 6, 7909.

(423) Belshaw, L.; Calegari, F.; Duffy, M. J.; Trabattoni, A.; Poletto, L.; Nisoli, M.; Greenwood, J. B. Observation of Ultrafast Charge Migration in an Amino Acid. J. Phys. Chem. Letters 2012, 3, 3751-3754.

(424) Plekan, O.; Feyer, V.; Richter, R.; Coreno, M.; Prince, C. K. Valence Photoionization and Photofragmentation of Aromatic Amino Acids. Mol. Phys. 2008, 106, 1143-1153.

(425) Mendive-Tapia, D.; Vacher, M.; Bearpark, M. J.; Robb, M. A. Coupled ElectronNuclear Dynamics: Charge Migration and Charge Transfer Initiated near a Conical Intersection. J. Chem. Phys. 2013, 139, 044110.

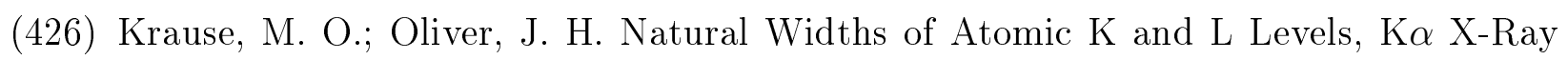
Lines and Several KLL Auger Lines. J. Phys. Chem. Ref. Data 1979, 8, 329-338. 
(427) Ikeura-Sekiguchi, H.; Sekiguchi, T. Femto- and Attosecond Electron Dynamics in 5'Guanosine Monophosphate Interface as Probed by Resonant Auger Spectroscopy. Surf. Interface Anal. 2010, 42, 1085-1088.

(428) Kuleff, A. I.; Cederbaum, L. S. Radiation Generated by the Ultrafast Migration of a Positive Charge Following the Ionization of a Molecular System. Phys. Rev. Lett. 2011, 106, 053001.

(429) Mendive-Tapia, D.; Vacher, M.; Bearpark, M. J.; Robb, M. A. Coupled ElectronNuclear Dynamics: Charge Migration and Charge Transfer Initiated near a Conical Intersection. J. Chem. Phys. 2013, 139, 044110.

(430) Kuleff, A. I.; Cederbaum, L. S. Charge Migration in Different Conformers of Glycine: The Role of Nuclear Geometry. Chem. Phys. 2007, 338, 320-328.

(431) Kuleff, A. I.; Dreuw, A. Theoretical Description of Charge Migration with a Single Slater-Determinant and Beyond. Journal of Chem. Phys. 2009, 130, 034102.

(432) Remacle, F.; Levine, R. D. Probing Ultrafast Purely Electronic Charge Migration in Small Peptides. Z. Phys. Chem. 2007, 221, 647-661.

(433) Hudock, H. R.; Levine, B. G.; Thompson, A. L.; Satzger, H.; Townsend, D.; Gador, N.; Ullrich, S.; Stolow, A.; ; Martínez, T. J. Ab Initio Molecular Dynamics and TimeResolved Photoelectron Spectroscopy of Electronically Excited Uracil and Thymine. J. Phys. Chem. A 2007, 111, 8500-8508.

(434) Stolow, A.; Underwood, J. G. In Advances in Chem. Phys.; Rice, S. A., Ed.; John Wiley \& Sons, Inc., 2008; pp 497-584.

(435) Tao, H.; Allison, T. K.; Wright, T. W.; Stooke, A. M.; Khurmi, C.; van Tilborg, J.; Liu, Y.; Falcone, R. W.; Belkacem, A.; Martinez, T. J. Ultrafast Internal Conversion in Ethylene. I. The Excited State Lifetime. J. Chem. Phys. 2011, 134, 244306. 
(436) Kuś, T.; Mignolet, B.; Levine, R. D.; Remacle, F. Pump and Probe of Ultrafast Charge Reorganization in Small Peptides: A Computational Study Through Sudden Ionizations. J. Phys. Chem. A 2013, 117, 10513-10525.

(437) Jenkins, A. J.; Vacher, M.; Bearpark, M. J.; Robb, M. A. Nuclear Spatial Delocalization Silences Electron Density Oscillations in 2-Phenyl-Ethyl-Amine (PEA) and 2-Phenylethyl-N,N-Dimethylamine (PENNA) Cations. J. Chem. Phys. 2016, 144, 104110.

(438) Vacher, M.; Meisner, J.; Mendive-Tapia, D.; Bearpark, M. J.; Robb, M. A. Electronic Control of Initial Nuclear Dynamics Adjacent to a Conical Intersection. J. Phys. Chem. A 2015, 119, 5165-5172.

(439) Stolow, A. Femtosecond Time-Resolved Photoelectron Spectroscopy of Polyatomic Molecules. Annu. Rev. Phys. Chem. 2003, 54, 89-119.

(440) Stolow, A.; Bragg, A. E.; Neumark, D. M. Femtosecond Time-Resolved Photoelectron Spectroscopy. Chemical Reviews 2004, 104, 1719-1758.

(441) Halász, G. J.; Perveaux, A.; Lasorne, B.; Robb, M. A.; Gatti, F.; Vibók, A. Simulation of Laser-Induced Quantum Dynamics of the Electronic and Nuclear Motion in the Ozone Molecule on the Attosecond Time Scale. Phys. Rev. A 2012, 86, 043426.

(442) Halász, G. J.; Perveaux, A.; Lasorne, B.; Robb, M. A.; Gatti, F.; Vibók, A. Coherence Revival During the Attosecond Electronic and Nuclear Quantum Photodynamics of the Ozone Molecule. Phys. Rev. A 2013, 88, 023425.

(443) Perveaux, A.; Lauvergnat, D.; Gatti, F.; Halász, G. J.; Vibók, A.; Lasorne, B. Monitoring the Birth of an Electronic Wavepacket in a Molecule with Attosecond TimeResolved Photoelectron Spectroscopy. J. Phys. Chem. A 2014, 118, 8773-8778. 
(444) Meyer, H.-D.; Manthe, U.; Cederbaum, L. The Multi-Configurational Time-Dependent Hartree Approach. Chem. Phys. Lett. 1990, 165, 73 - 78.

(445) Manthe, U.; Meyer, H.; Cederbaum, L. S. Wave-Packet Dynamics within the Multiconfiguration Hartree Framework: General Aspects and Application to NOCl. J. Chem. Phys. 1992, 97, 3199-3213.

(446) Beck, M.; Jäckle, A.; Worth, G.; Meyer, H.-D. The Multiconfiguration TimeDependent Hartree (MCTDH) Method: a Highly Efficient Algorithm for Propagating Wavepackets. Phys. Rep. 2000, 324, 1-105.

(447) Perveaux, A.; Lauvergnat, D.; Lasorne, B.; Gatti, F.; Robb, M. A.; Halász, G. J.; Vibók, A. Attosecond Electronic and Nuclear Quantum Photodynamics of Ozone: Time-Dependent Dyson Orbitals and Dipole. J. Phys. B: At., Mol. Opt. Phys.: At., Mol. Opt. Phys. 2014, 47, 124010.

(448) Seideman, T. Revival Structure of Aligned Rotational Wave Packets. Phys. Rev. Lett. 1999, 83, 4971-4974.

(449) Rosca-Pruna, F.; Vrakking, M. J. J. Experimental Observation of Revival Structures in Picosecond Laser-Induced Alignment of $I_{2}$. Phys. Rev. Lett. 2001, 87, 153902.

(450) Lein, M.; Hay, N.; Velotta, R.; Marangos, J. P.; Knight, P. L. Role of the Intramolecular Phase in High-Harmonic Generation. Phys. Rev. Lett. 2002, 88, 183903.

(451) Itatani, J.; Zeidler, D.; Levesque, J.; Spanner, M.; Villeneuve, D. M.; Corkum, P. B. Controlling High Harmonic Generation with Molecular Wave Packets. Phys. Rev. Lett. 2005, 94, 123902.

(452) Kanai, T.; Minemoto, S.; Sakai, H. Quantum Interference During High-Order Harmonic Generation from Aligned Molecules. Nature 2005, 435, 470-474. 
(453) Itatani, J.; Levesque, J.; Zeidler, D.; Niikura, H.; Pepin, H.; Kieffer, J. C.; Corkum, P. B.; Villeneuve, D. M. Tomographic Imaging of Molecular Orbitals. Nature 2004, 432, 867-871.

(454) Boutu, W.; Haessler, S.; Merdji, H.; Breger, P.; Waters, G.; Stankiewicz, M.; Frasinski, L. J.; Taieb, R.; Caillat, J.; Maquet, A.; et al., Coherent Control of Attosecond Emission from Aligned Molecules. Nat. Phys. 2008, 4, 545-549.

(455) Vozzi, C.; Negro, M.; Calegari, F.; Sansone, G.; Nisoli, M.; De Silvestri, S.; Stagira, S. Generalized Molecular Orbital Tomography. Nat. Phys. 2011, 7, 822-826.

(456) Negro, M.; Devetta, M.; Facciala, D.; De Silvestri, S.; Vozzi, C.; Stagira, S. Highorder harmonic spectroscopy for molecular imaging of polyatomic molecules. Faraday Discuss. 2014, 171, 133-143.

(457) Haessler, S.; Caillat, J.; Boutu, W.; Giovanetti-Teixeira, C.; Ruchon, T.; Auguste, T.; Diveki, Z.; Breger, P.; Maquet, A.; Carre, B.; et al., Attosecond Imaging of Molecular Electronic Wavepackets. Nat. Phys. 2010, 6, 200-206.

(458) Smirnova, O.; Mairesse, Y.; Patchkovskii, S.; Dudovich, N.; Villeneuve, D.; Corkum, P.; Ivanov, M. Y. High Harmonic Interferometry of Multi-Electron Dynamics in Molecules. Nature 2009, 460, 972-977.

(459) Wörner, H. J.; Bertrand, J. B.; Hockett, P.; Corkum, P. B.; Villeneuve, D. M. Controlling the Interference of Multiple Molecular Orbitals in High-Harmonic Generation. Phys. Rev. Lett. 2010, 104, 233904.

(460) Jin, C.; Bertrand, J. B.; Lucchese, R. R.; Wörner, H. J.; Corkum, P. B.; Villeneuve, D. M.; Le, A.-T.; Lin, C. D. Intensity Dependence of Multiple Orbital Contributions and Shape Resonance In High-Order Harmonic Generation of Aligned $\mathrm{N}_{2}$ Molecules. Phys. Rev. A 2012, 85, 013405. 
(461) Meckel, M.; Comtois, D.; Zeidler, D.; Staudte, A.; Pavičić, D.; Bandulet, H. C.; Pépin, H.; Kieffer, J. C.; Dörner, R.; Villeneuve, D. M.; et al., Laser-Induced Electron Tunneling and Diffraction. Science 2008, 320, 1478-1482.

(462) Blaga, C. I.; Xu, J.; DiChiara, A. D.; Sistrunk, E.; Zhang, K.; Agostini, P.; Miller, T. A.; DiMauro, L. F.; Lin, C. D. Imaging Ultrafast Molecular Dynamics with Laser-Induced Electron Diffraction. Nature 2012, 483, 194-197.

(463) Pullen, M. G.; Wolter, B.; Le, A.-T.; Baudisch, M.; Hemmer, M.; Senftleben, A.; Schroter, C. D.; Ullrich, J.; Moshammer, R.; Lin, C. D.; et al., Imaging an Aligned Polyatomic Molecule with Laser-Induced Electron Diffraction. Nat. Commun. 2015, 6,7262 .

(464) Pullen, M. G.; Wolter, B.; Le, A.-T.; Baudisch, M.; Sclafani, M.; Pires, H.; Schroter, C. D.; Ullrich, J.; Moshammer, R.; Pfeifer, T.; et al., Influence of Orbital Symmetry on Diffraction Imaging with Rescattering Electron Wave Packets. Nat. Commun. 2016, \%, 11922.

(465) Wolter, B.; Pullen, M. G.; Le, A.-T.; Baudisch, M.; Doblhoff-Dier, K.; Senftleben, A.; Hemmer, M.; Schröter, C. D.; Ullrich, J.; Pfeifer, T.; et al., Ultrafast Electron Diffraction Imaging of Bond Breaking in di-Ionized Acetylene. Science 2016, 354, 308-312.

(466) Gabor, D. Nobel Lectures, Physics 1971-1980; World Scientific Pubishing, Singapore, 1992.

(467) Huismans, Y.; Rouzée, A.; Gijsbertsen, A.; Jungmann, J. H.; Smolkowska, A. S.; Logman, P. S. W. M.; Lépine, F.; Cauchy, C.; Zamith, S.; Marchenko, T.; et al., Time-Resolved Holography with Photoelectrons. Science 2011, 331, 61-64.

(468) Popruzhenko, S.; Bauer, D. Strong Field Approximation for Systems with Coulomb Interaction. J. Mod. Opt. 2008, 55, 2573-2589. 
(469) Meckel, M.; Staudte, A.; Patchkovskii, S.; Villeneuve, D. M.; Corkum, P. B.; Dorner, R.; Spanner, M. Signatures of the Continuum Electron Phase in Molecular Strong-Field Photoelectron Holography. Nat. Phys. 2014, 10, 594-600.

(470) Petretti, S.; Vanne, Y. V.; Saenz, A.; Castro, A.; Decleva, P. Alignment-Dependent Ionization of $\mathrm{N}_{2}, \mathrm{O}_{2}$, and $\mathrm{CO}_{2}$ in Intense Laser Fields. Phys. Rev. Lett. 2010, 104, 223001.

(471) Boguslavskiy, A. E.; Mikosch, J.; Gijsbertsen, A.; Spanner, M.; Patchkovskii, S.; Gador, N.; Vrakking, M. J. J.; Stolow, A. The Multielectron Ionization Dynamics Underlying Attosecond Strong-Field Spectroscopies. Science 2012, 335, 1336-1340.

(472) Mikosch, J.; Boguslavskiy, A. E.; Wilkinson, I.; Spanner, M.; Patchkovskii, S.; Stolow, A. Channel- and Angle-Resolved Above Threshold Ionization in the Molecular Frame. Phys. Rev. Lett. 2013, 110, 023004.

(473) Schulz, C. P.; Birkner, S.; Furch, F. J.; Anderson, A.; Mikosch, J.; Schell, F.; Vrakking, M. J. J. Strong field ionization of small hydrocarbon chains with full 3D momentum analysis. Journal of Physics: Conference Series 2015, 635, 112122.

(474) Yao, J.; Li, G.; Jia, X.; Hao, X.; Zeng, B.; Jing, C.; Chu, W.; Ni, J.; Zhang, H.; Xie, H.; et al., Alignment-Dependent Fluorescence Emission Induced by Tunnel Ionization of Carbon Dioxide from Lower-Lying Orbitals. Phys. Rev. Lett. 2013, 111, 133001.

(475) Pavičić, D.; Lee, K. F.; Rayner, D. M.; Corkum, P. B.; Villeneuve, D. M. Direct Measurement of the Angular Dependence of Ionization for $\mathrm{N}_{2}, \mathrm{O}_{2}$, and $\mathrm{CO}_{2}$ in Intense Laser Fields. Phys. Rev. Lett. 2007, 98, 243001.

(476) Zhao, S.-F.; Jin, C.; Le, A.-T.; Jiang, T. F.; Lin, C. D. Analysis of Angular Dependence of Strong-Field Tunneling Ionization for $\mathrm{CO}_{2}$. Phys. Rev. A 2009, 80, 051402. 
(477) Zhao, S.-F.; Jin, C.; Le, A.-T.; Jiang, T. F.; Lin, C. D. Determination of Structure Parameters in Strong-Field Tunneling Ionization Theory of Molecules. Phys. Rev. A 2010, 81, 033423. 